Supporting Information for

\title{
Synthesis of Cyclotriveratrylene-Sucrose-Based Capsules
}

Lukasz Szyszka, Piotr Cmoch, Aleksandra Butkiewicz, Mykhaylo A. Potopnyk and Sławomir Jarosz

Institute of Organic Chemistry, Polish Academy of Sciences, ul. Kasprzaka 44/52, 01-224 Warsaw, Poland 


\section{Contents}

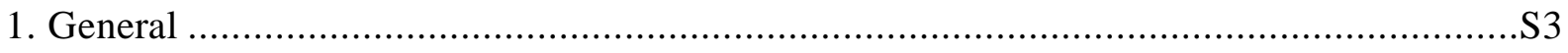

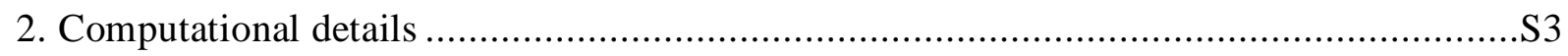

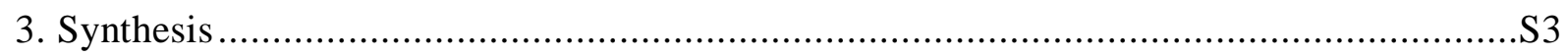

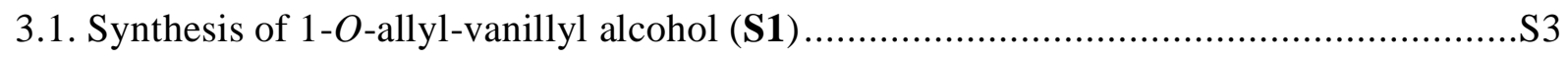

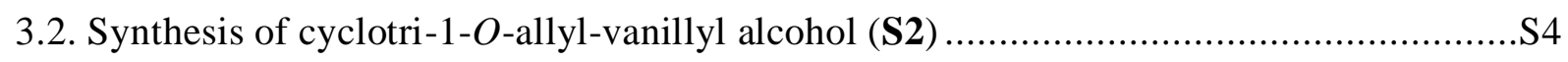

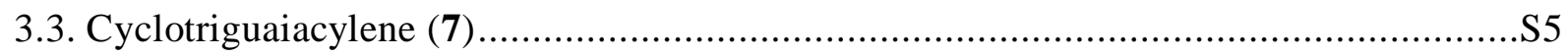

3.4. 1',6,6'-Tri-O-tert-butyldimethylsilylsucrose (S3) …………………......................... 5

3.5. 1',6,6'-Tri- $O$-tert-butyldimethylsilyl-2,3,3',4,4'-penta- $O$-benzylsucrose (2) ..................S6

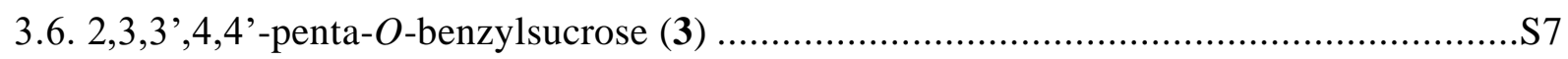

3.7. 2,3,3',4,4'-Penta-O-benzyl-1',6,6'-tri- $O$-(2-tert-butoxy-2-oxoethyl)-sucrose (4) …........S7

3.8. 2,3,3',4,4'-Penta- $O$-benzyl-1',6,6'-tri- $O$-(2-hydroxyethyl)-sucrose (5) …......................S8

3.9. 2,3,3',4,4'-Penta-O-benzyl-1',6,6'-tri- $O$-(2-iodoethyl)-sucrose (6) ...............................S9

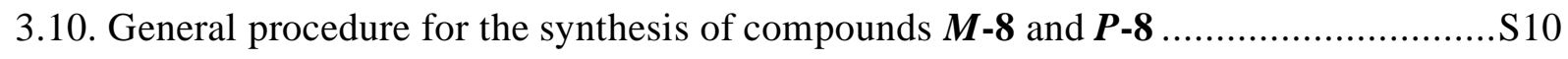

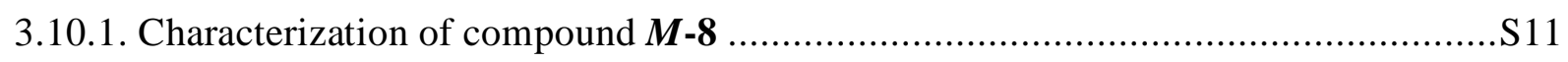

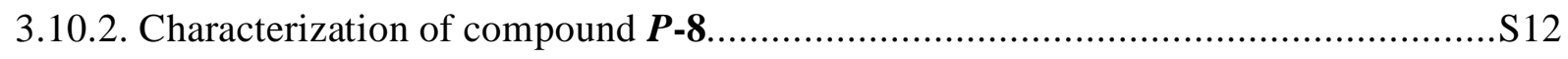

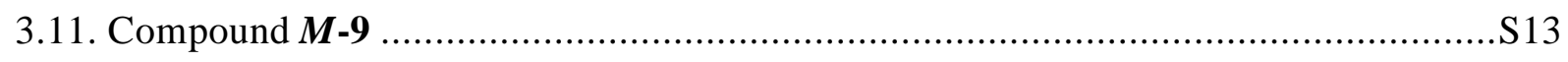

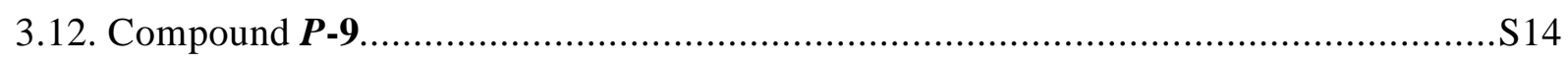

Figure S1.Comparison of experimental UV (top) and ECD (bottom) spectra of compounds $\boldsymbol{M}$ 8 and $\boldsymbol{P}-\mathbf{8}$ in $\mathrm{MeCN}$ solution with the simulated curves calculated at CAM-

B3LYP/SVP/PCM(MeCN) level of theory.

Table S1. Calculated at CAM-B3LYP/SVP/PCM $\left(\mathrm{CH}_{3} \mathrm{CN}\right)$ level of theory relative energies

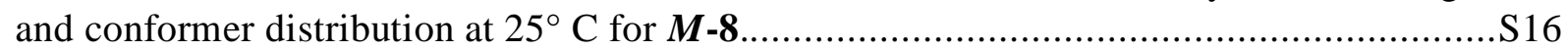

Cartesian coordinates for individual conformers of compound $\boldsymbol{M - 8}$................................S16

Table S2. Calculated at CAM-B3LYP/SVP/PCM $\left(\mathrm{CH}_{3} \mathrm{CN}\right)$ level of theory relative energies

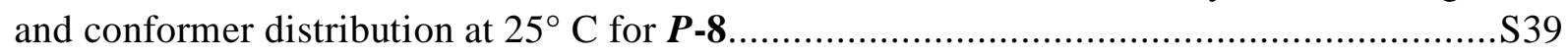

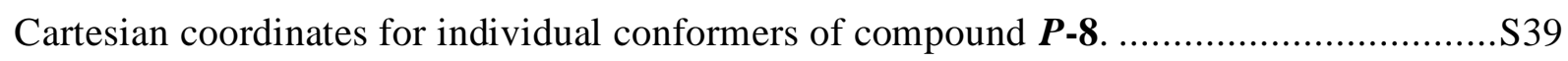

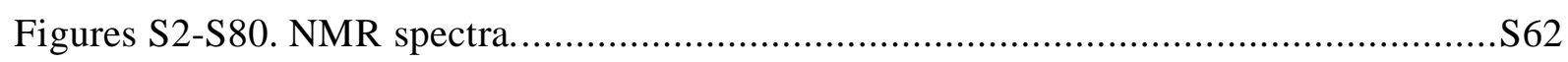

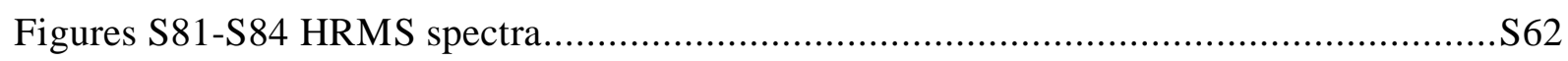




\section{General}

Commercially available reagents and solvents were used as received. TLC was performed on Merck silica gel $60 \mathrm{~F}_{254}$ plates. Compounds were purified using automatic flash chromatography system Knauer with UV and ELSD detection and Grace Resolv or Reveleris cartridges. The NMR spectra were recorded with Bruker Avance II $400 \mathrm{MHz}$ (at $400 \mathrm{MHz}$ and $100 \mathrm{MHz}$ for ${ }^{1} \mathrm{H}$ and ${ }^{13} \mathrm{C}$ NMR spectra, respectively), Varian VNMRS $500 \mathrm{MHz}$ (at $500 \mathrm{MHz}$ and $125 \mathrm{MHz}$ for ${ }^{1} \mathrm{H}$ and ${ }^{13} \mathrm{C}$ NMR spectra, respectively) or Varian VNMRS $600 \mathrm{MHz}$ (at 600 $\mathrm{MHz}$ and $150 \mathrm{MHz}$ for ${ }^{1} \mathrm{H}$ and ${ }^{13} \mathrm{C}$ NMR spectra, respectively) spectrometers for solutions in $\mathrm{CDCl}_{3}$, acetone- $d_{6}, \mathrm{CD}_{3} \mathrm{OD}$ or $\mathrm{CD}_{3} \mathrm{CN}$, and $\mathrm{TMS}$ as the internal standard at $298 \mathrm{~K}$. All significant resonances were assigned by COSY $\left({ }^{1} \mathrm{H}-{ }^{1} \mathrm{H}\right)$, HSQC $\left({ }^{1} \mathrm{H}_{-}{ }^{13} \mathrm{C}\right)$ and $\mathrm{HMBC}\left({ }^{1} \mathrm{H}_{-}{ }^{13} \mathrm{C}\right)$ correlations. Mass spectra were measured with Synapt G2-S HDMS (Waters Inc) mass spectrometer equipped with an electrospray ion source and q-TOF type mass analyzer. Optical rotations were measured with a Jasco P 2000 apparatus in $\mathrm{CHCl}_{3}$ or $\mathrm{MeOH}$ with a sodium lamp at r.t. (c 1). Elemental analyses were obtained with a Perkin-Elmer $2400 \mathrm{CHN}$ analyzer. The ECD and UV spectra of $\boldsymbol{P} \mathbf{- 8}$ and $\boldsymbol{M - 8}$ were recorded at room temperature in $\mathrm{MeCN}$ (for UV-spectroscopy, Fluka) on a Jasco J-715 spectropolarimeter with concentrations of $7.0 \times 10^{-5} \mathrm{M}$ in $0.1 \mathrm{~cm}$ quartz cell. All spectra were recorded using a $100 \mathrm{~nm} / \mathrm{min}$ scanning speed, a step size of $0.2 \mathrm{~nm}$, a bandwidth of $1 \mathrm{~nm}$, a response time of $0.5 \mathrm{~s}$, and an accumulation of 5 scans. The baseline of the spectra was corrected by subtracting the spectrum of the pure solvent recorded under the same conditions.

\section{Computational details}

First, the conformational analysis of $\boldsymbol{M - 8}$ and $\boldsymbol{P - 8}$ was performed by using Conflex 7 program ${ }^{1}$ with the MMFF94s force field and $5 \mathrm{kcal} / \mathrm{mol}$ energy window. Next, ten most stable conformers for each $\boldsymbol{P - 8}$ and $\boldsymbol{M - 8}$ molecules were optimized at the CAM-B3LYP/SVP/PCM $(\mathrm{MeCN})$ level. $^{2}$ The stable structures were found by ascertaining that all the harmonic frequencies were real and the relative abundances were calculated on the $\Delta \mathrm{G}$ values relative to the most stable conformer. The Electronic Absorption EA and ECD spectra were calculated for all conformers with a population higher than $1 \%$ taking into account the lowest 150 singlet states. Finally, the EA and ECD spectra were averaged taking into account the $\Delta \mathrm{G}$ values at room temperature and plotted with Gaussian bandshape and $0.20 \mathrm{eV}$ half-height width. All calculations were performed using the Gaussian 16 package of programs. ${ }^{3}$

\section{Synthesis}

\subsection{Synthesis of 1-O-allyl-vanillyl alcohol (S1)}<smiles>COc1cc(CO)ccc1O</smiles><smiles>C=CCBr</smiles><smiles>C=CCOc1ccc(CO)cc1OC</smiles>

1-O-allyl-vanillyl alcohol (S1) was prepared according to the literature procedure. ${ }^{4}$ To the solution of vanillyl alcohol $(5 \mathrm{~g}, 0.032 \mathrm{~mol})$ in acetone $(25 \mathrm{~mL}), \mathrm{K}_{2} \mathrm{CO}_{3}(4.5 \mathrm{~g}, 0.032 \mathrm{~mol})$ 
and allyl bromide $(3.3 \mathrm{~mL}, 0.038 \mathrm{~mol})$ were added. The mixture was stirred under reflux overnight. Afterwards, acetone was removed under vacuum and the residue was partitioned between $\mathrm{CH}_{2} \mathrm{Cl}_{2}(50 \mathrm{~mL})$ and water $(30 \mathrm{~mL})$. The organic phase was then dried over $\mathrm{Na}_{2} \mathrm{SO}_{4}$ and concentrated. The crude product was purified by crystallization from methyl tert-butyl ether/hexanes obtaining S1 (6 g, $0.031 \mathrm{~mol}, 95 \%)$ as white crystals. ${ }^{1} \mathrm{H}$ NMR (400 MHz, $\left.\mathrm{CDCl}_{3}\right) \delta: 6.93(\mathrm{~s}, 1 \mathrm{H}), 6.85(\mathrm{~s}, 2 \mathrm{H}), 6.02-6.13(\mathrm{~m}, 1 \mathrm{H}), 5.39$ (dd, $\left.J=1.5 \mathrm{~Hz}, 17.3 \mathrm{~Hz}, 1 \mathrm{H}\right)$, $5.28(\mathrm{dd}, J=1.4 \mathrm{~Hz}, 10.5 \mathrm{~Hz}, 1 \mathrm{H}), 4.58-4.62(\mathrm{~m}, 4 \mathrm{H}), 3.88(\mathrm{~s}, 3 \mathrm{H}), 1.76(\mathrm{~s}, \mathrm{OH}) \mathrm{ppm}$. ${ }^{13} \mathrm{C}\{\mathrm{H}\} \mathrm{NMR}\left(100 \mathrm{MHz}, \mathrm{CDCl}_{3}\right) \delta: 149.62,147.54,134.01,133.31,119.26,117.89,113.44$, $110.88,69.95,65.24,55.88 \mathrm{ppm}$. HRMS (ESI-TOF) calcd for $\mathrm{C}_{11} \mathrm{H}_{14} \mathrm{O}_{3} \mathrm{Na}[\mathrm{M}+\mathrm{Na}]^{+}$: 217.0841, found: 217.0836 . ${ }^{1} \mathrm{H}$ and ${ }^{13} \mathrm{C}$ NMR spectra were consistent with those previously reported in the literature. ${ }^{4}$

\subsection{Synthesis of cyclotri-1-O-allyl-vanillyl alcohol (S2)}

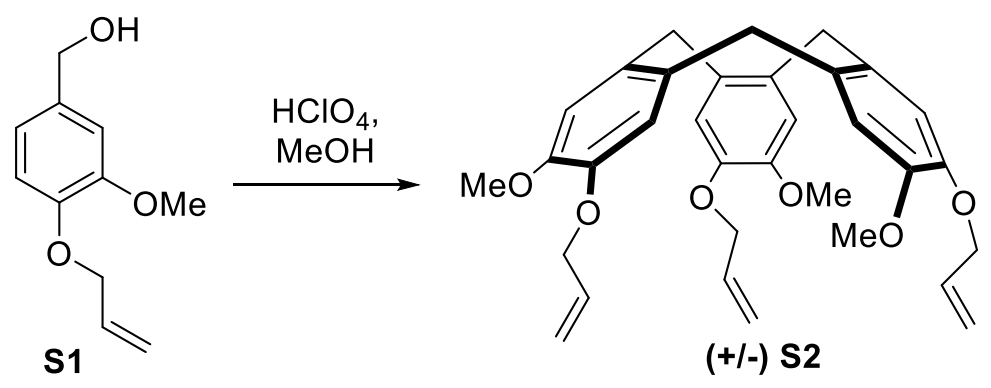

Racemic compound S2 was prepared according to a modified literature procedure. ${ }^{4}$ To the cooled to $0{ }^{\circ} \mathrm{C}$ solution of $\mathbf{S 1}(11.1 \mathrm{~g}, 0.057 \mathrm{~mol})$ in $\mathrm{MeOH}(66.6 \mathrm{~mL}), 65 \%$ aqueous $\mathrm{HClO}_{4}$ $(33.3 \mathrm{~mL})$ was added dropwise, the reaction mixture was allowed to reach room temperature, and stirred for $20 \mathrm{~h}$. Then it was diluted with $\mathrm{CH}_{2} \mathrm{Cl}_{2}(60 \mathrm{~mL})$, cooled to $0{ }^{\circ} \mathrm{C}$, and $50 \%$ aq. $\mathrm{NaOH}$ was carefully added dropwise as its color changed from pink to yellow. The layers were separated and the aqueous one was washed with $\mathrm{CH}_{2} \mathrm{Cl}_{2}(3 \times 30 \mathrm{~mL})$. Combined organic phases were washed with water $(80 \mathrm{~mL})$, then brine $(50 \mathrm{~mL})$, dried over $\mathrm{Na}_{2} \mathrm{SO}_{4}$, and concentrated under reduced pressure. The resulting residue was suspended in $\mathrm{Et}_{2} \mathrm{O}(50 \mathrm{~mL})$ and stirred for $4 \mathrm{~h}$ before being filtered off. The solid was washed with $\mathrm{Et}_{2} \mathrm{O}(3 \times 10 \mathrm{~mL})$ and dried in high vacuum. Pure racemic compound 12 (5.22 g, $0.01 \mathrm{~mol}, 52 \%)$ was obtained as a white solid. ${ }^{1} \mathrm{H}$ NMR $\left(400 \mathrm{MHz}, \mathrm{CDCl}_{3}\right) \delta$ : $6.85(\mathrm{~s}, 3 \mathrm{H}), 6.79(\mathrm{~s}, 3 \mathrm{H}), 6.01-6.11(\mathrm{~m}, 3 \mathrm{H})$, $5.37(\mathrm{~d}, J=17.2 \mathrm{~Hz}, 3 \mathrm{H}), 5.25(\mathrm{~d}, J=10.7 \mathrm{~Hz}, 3 \mathrm{H}), 4.74(\mathrm{~d}, J=13.7 \mathrm{~Hz}, 3 \mathrm{H}), 4.53-4.64(\mathrm{~m}$, $6 \mathrm{H}) 3.83(\mathrm{~s}, 9 \mathrm{H}), 3.51(\mathrm{~d}, J=13.8 \mathrm{~Hz}, 3 \mathrm{H}) \mathrm{ppm} .{ }^{13} \mathrm{C}\{\mathrm{H}\} \mathrm{NMR}\left(100 \mathrm{MHz}, \mathrm{CDCl}_{3}\right) \delta: 148.24$, $146.79,133.77,132.36,131.77,117.47,115.66,113.69,70.23,56.13,36.52$ ppm. HRMS (ESI-TOF) calcd for $\mathrm{C}_{33} \mathrm{H}_{36} \mathrm{O} 6 \mathrm{Na}[\mathrm{M}+\mathrm{Na}]^{+}: 551.2410$, found: $551.2407 .{ }^{1} \mathrm{H}$ and ${ }^{13} \mathrm{C} \mathrm{NMR}$ spectra were consistent with those previously reported in the literature. ${ }^{4}$ 


\subsection{Cyclotriguaiacylene (7)}

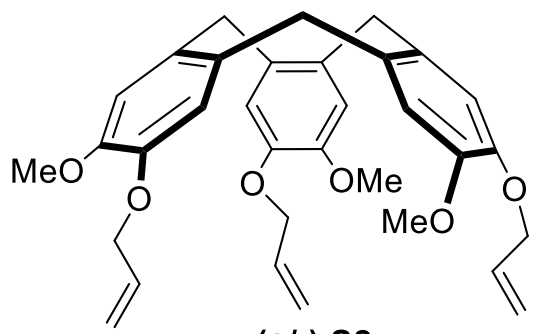

$(+/-) \mathbf{S 2}$

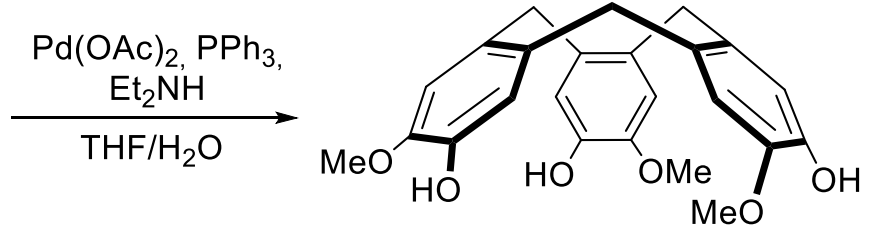

$(+/-) 7$

Racemic compound 7 was prepared according to a modified literature procedure. ${ }^{5}$ This reaction was conducted under an argon atmosphere. To a solution of $\mathbf{S 2}(9.0 \mathrm{~g}, 0.017 \mathrm{~mol})$ in THF (225 mL) and $\mathrm{H}_{2} \mathrm{O}$ (45 mL), $\mathrm{Pd}(\mathrm{OAc})_{2}(572.5 \mathrm{mg}, 2.55 \mathrm{mmol}), \mathrm{PPh}_{3}(2.0 \mathrm{~g}, 7.65 \mathrm{mmol})$, and diethylamine $(90 \mathrm{~mL})$ were added, and the mixture was stirred for $4 \mathrm{~h}$ at $80{ }^{\circ} \mathrm{C}$. After removal of solvents under vacuum, ethyl acetate $(100 \mathrm{~mL})$ was added, the dark insoluble residue was filtered and washed with ethyl acetate. The resulting filtrate was washed with water $(100 \mathrm{~mL})$ and then with brine $(100 \mathrm{~mL})$. Combined organic phases were dried over $\mathrm{Na}_{2} \mathrm{SO}_{4}$ and concentrated. The resulting yellow solid was washed with ether $(3 \times 50 \mathrm{~mL})$ and recrystallized from dichloromethane/hexanes giving the desired product 7 (4.52 g, 0.011 mol, $65 \%)$ as off-white powder. ${ }^{1} \mathrm{H}$ NMR $\left(500 \mathrm{MHz}\right.$, acetone- $\left.d_{6}\right): \delta=6.99(\mathrm{~s}, 3 \mathrm{H}), 6.93(\mathrm{~s}, 3 \mathrm{H})$, $4.74(\mathrm{~d}, J=13.6 \mathrm{~Hz}, 3 \mathrm{H}), 3.80(\mathrm{~s}, 9 \mathrm{H}), 3.50(\mathrm{~d}, J=13.6 \mathrm{~Hz}, 3 \mathrm{H}) \mathrm{ppm} .{ }^{13} \mathrm{C}\{\mathrm{H}\} \mathrm{NMR}(125$ $\mathrm{MHz}$, acetone-d6): $\delta=146.81,145.83,133.86,132.00,117.15,114.24,56.54,36.47 \mathrm{ppm}$. HRMS (ESI-TOF) calcd for $\mathrm{C}_{24} \mathrm{H}_{24} \mathrm{O}_{6} \mathrm{Na}[\mathrm{M}+\mathrm{Na}]^{+}: 431.1471$, found: $431.1472 .{ }^{1} \mathrm{H}$ and ${ }^{13} \mathrm{C}$ NMR spectra were consistent with those previously reported in the literature. ${ }^{5}$

\subsection{1',6,6'-Tri-O-tert-butyldimethylsilylsucrose (S3)}
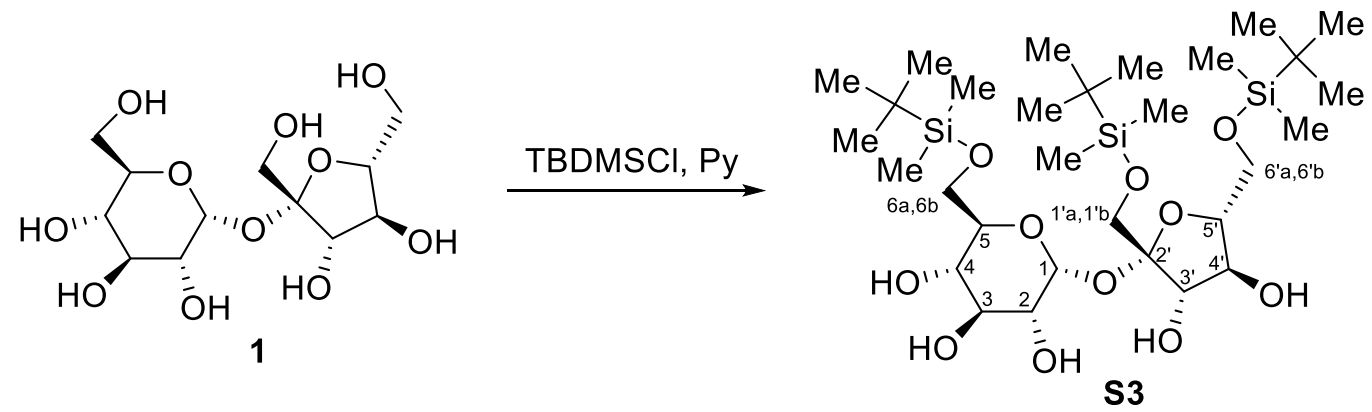

Sucrose (1, $10 \mathrm{~g}, 0.029 \mathrm{~mol})$ was dissolved in boiling pyridine $(200 \mathrm{~mL})$. After cooling to room temperature, a solution of tert-butyldimethylsilyl chloride $(16.5 \mathrm{~g}, 0.109 \mathrm{~mol})$ in pyridine $(100 \mathrm{~mL})$ was added dropwise during $30 \mathrm{~min}$ and the mixture was stirred for $24 \mathrm{~h}$ at $60{ }^{\circ} \mathrm{C}$. Pyridine was evaporated and the products were isolated by column chromatography (hexanes/ethyl acetate $=1: 2$ to $100 \%$ ethyl acetate) to afford compound S3 (13.0 g, 0.019 mol, $65 \%)$ as a white solid. $[\alpha]_{\mathrm{D}}=+50.4(\mathrm{MeOH}) .{ }^{1} \mathrm{H}$ NMR $\left(600 \mathrm{MHz}, \mathrm{CD}_{3} \mathrm{OD}\right): \delta=5.39(\mathrm{~d}$, $\left.J_{1,2}=3.9 \mathrm{~Hz}, 1 \mathrm{H}, \mathrm{H}-1\right), 4.17$ (d, $\left.J_{3^{\prime}, 4^{\prime}}=8.7 \mathrm{~Hz}, 1 \mathrm{H}, \mathrm{H}-3^{\prime}\right), 3.87-3.92$ (m, 2H, H-4', H-6'a),

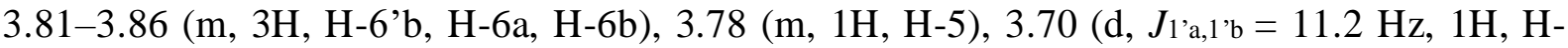
1'a), 3.68 (m, 1H, H-5'), 3.66 (d, 1H, H-1'b), 3.61 (dd, $J_{3,2}=9.8 \mathrm{~Hz}, J_{3,4}=9.0 \mathrm{~Hz}, 1 \mathrm{H}, \mathrm{H}-3$ ), $3.54\left(\mathrm{dd}, J_{4,5}=9.8 \mathrm{~Hz}, 1 \mathrm{H}, \mathrm{H}-4\right), 3.32(\mathrm{dd}, 1 \mathrm{H}, \mathrm{H}-2), 0.912\left(\mathrm{~s}, 9 \mathrm{H},{ }^{t} \mathrm{Bu}\right), 0.909\left(\mathrm{~s}, 9 \mathrm{H},{ }^{t} \mathrm{Bu}\right)$, $0.900\left(\mathrm{~s}, 9 \mathrm{H},{ }^{t} \mathrm{Bu}\right), 0.81-0.95\left(\mathrm{~m}, 18 \mathrm{H}, 6 \times \mathrm{SiCH}_{3}\right) \mathrm{ppm} .{ }^{13} \mathrm{C}\{\mathrm{H}\} \mathrm{NMR}\left(150 \mathrm{MHz}, \mathrm{CD}_{3} \mathrm{OD}\right): \delta$ 
$=105.55$ (C-2'), 93.25 (C-1), 83.86 (C-5'), 77.47 (C-3'), 75.80 (C-4'), 74.98 (C-3), 74.26 (C5), 73.21 (C-2), 71.34 (C-4), 65.86 (C-6'), 64.27 (C-1'), 64.00 (C-6), 26.61 (triple intensity, $3 \mathrm{C}-{ }^{t} \mathrm{Bu}$ ), 26.50 (triple intensity, 3C- ${ }^{t} \mathrm{Bu}$ ), 26.44 (triple intensity, $\left.3 \mathrm{C}-{ }^{t} \mathrm{Bu}\right), 19.44\left(\mathrm{C}_{\text {quat }},{ }^{t} \mathrm{Bu}\right.$ ), $19.21\left(\mathrm{C}_{\text {quat }},{ }^{t} \mathrm{Bu}\right), 19.18\left(\mathrm{C}_{\text {quat }},{ }^{t} \mathrm{Bu}\right),-4.84,-4.84,-4.93,-4.97,-5.05,-5.22\left(6 \times \mathrm{CH}_{3}-\mathrm{Si}\right) \mathrm{ppm}$. HRMS (ESI-TOF) calcd for $\mathrm{C}_{30} \mathrm{H}_{64} \mathrm{O}_{11} \mathrm{Si}_{3} \mathrm{Na}[\mathrm{M}+\mathrm{Na}]^{+}:$707.3654, found: 707.3632. Anal. calcd for $\mathrm{C}_{30} \mathrm{H}_{64} \mathrm{O}_{11} \mathrm{Si}_{3}$ : C, 52.60; H, 9.42; found: C, 52.59; H, 9.27.

\subsection{1',6,6'-Tri- $O$-tert-butyldimethylsilyl-2,3,3',4,4'-penta- $O$-benzylsucrose (2)}
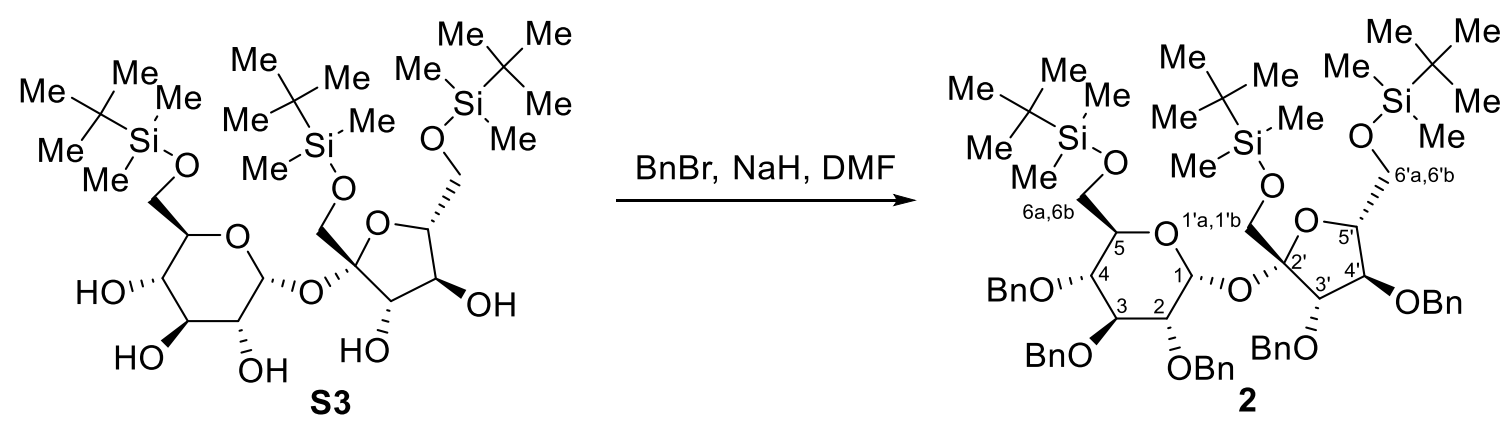

Sodium hydride (60\% dispersion in mineral oil, $6.47 \mathrm{~g}, 161.7 \mathrm{mmol}$ ) was added portionwise at $0-5{ }^{\circ} \mathrm{C}$ to a stirred solution of compound $\mathbf{S 3}(10.55 \mathrm{~g}, 15.4 \mathrm{mmol})$ in DMF (385 mL) and the mixture was stirred at room temperature for $30 \mathrm{~min}$. Benzyl bromide $(19.2 \mathrm{~mL}, 161.7$ mmol) was added dropwise during $20 \mathrm{~min}$ and the mixture was stirred overnight. Excess of hydride was decomposed by careful addition of methanol $(20 \mathrm{~mL})$ and the mixture was partitioned between saturated aq. $\mathrm{NH}_{4} \mathrm{Cl}(200 \mathrm{~mL})$ and diethyl ether $(300 \mathrm{~mL})$. The layers were separated and the aqueous one was extracted with diethyl ether $(3 \times 200 \mathrm{~mL})$. Combined organic solutions were dried over $\mathrm{Na}_{2} \mathrm{SO}_{4}$, concentrated, and the resulting residue was purified by column chromatography (hexanes $/ \mathrm{CH}_{2} \mathrm{Cl}_{2}=4: 1$ to $1: 1$ ) to afford pure product 2 $(9.6 \mathrm{~g}, 55 \%)$ as a colorless oil. $[\alpha]_{\mathrm{D}}=+30.3\left(\mathrm{CHCl}_{3}\right) .{ }^{1} \mathrm{H} \mathrm{NMR}\left(600 \mathrm{MHz}, \mathrm{CDCl}_{3}\right): \delta=7.37-$ $7.53(\mathrm{~m}, 25 \mathrm{H}, 25 \times \mathrm{H}-\mathrm{Ph}), 6.06\left(\mathrm{~d}, J_{1,2}=3.7 \mathrm{~Hz}, 1 \mathrm{H}, \mathrm{H}-1\right), 5.07(\mathrm{~d}, J=10.7 \mathrm{~Hz}, 1 \mathrm{H}$, benzylic $\mathrm{H}), 5.01(\mathrm{~d}, J=11.0 \mathrm{~Hz}, 1 \mathrm{H}$, benzylic H), $4.93(\mathrm{~d}, J=10.7 \mathrm{~Hz}, 1 \mathrm{H}$, benzylic H), $4.91(\mathrm{~d}, J=$ $11.8 \mathrm{~Hz}, 1 \mathrm{H}$, benzylic H), $4.90(\mathrm{~d}, J=11.1 \mathrm{~Hz}, 1 \mathrm{H}$, benzylic $\mathrm{H}), 4.90(\mathrm{~d}, J=11.0 \mathrm{~Hz}, 1 \mathrm{H}$, benzylic H), $4.84(\mathrm{~d}, J=11.0 \mathrm{~Hz}, 1 \mathrm{H}$, benzylic $\mathrm{H}), 4.79(\mathrm{~d}, J=11.7 \mathrm{~Hz}, 1 \mathrm{H}$, benzylic $\mathrm{H}), 4.74$ $\left(\mathrm{d}, J=11.0 \mathrm{~Hz}, 1 \mathrm{H}\right.$, benzylic H), $4.69(\mathrm{~d}, J=11.0 \mathrm{~Hz}, 1 \mathrm{H}$, benzylic $\mathrm{H}), 4.65\left(\mathrm{~d}, J_{3^{\prime}, 4^{\prime}}=8.2\right.$

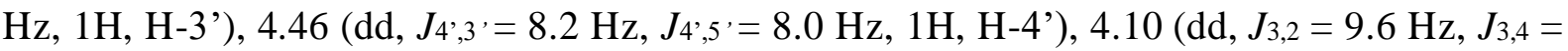
9.1 Hz, 1H, H-3), 4.04-4.07 (m, 2H, H-5, H-6'a), 3.97-4.01 (m, 2H, H-5', H-6'b), 3.91 (d, $J_{1}{ }^{\prime} \mathrm{a}, 1^{\prime} \mathrm{b}=11.1 \mathrm{~Hz}, 1 \mathrm{H}, \mathrm{H}-1$ 'a), $3.86\left(\mathrm{dd}, J_{4,5}=10.0 \mathrm{~Hz}, J_{4,3}=9.1 \mathrm{~Hz}, 1 \mathrm{H}, \mathrm{H}-4\right), 3.78\left(\mathrm{~d}, J_{1}{ }^{\prime} \mathrm{b}, 1^{\prime} \mathrm{a}\right.$ $=11.1 \mathrm{~Hz}, 1 \mathrm{H}, \mathrm{H}-1$ 'b), $3.73\left(\mathrm{dd}, J_{6 \mathrm{a}, 6 \mathrm{~b}}=11.7 \mathrm{~Hz}, J_{6 \mathrm{a}, 5}=2.2 \mathrm{~Hz}, 1 \mathrm{H}, \mathrm{H}-6 \mathrm{a}\right), 3.65\left(\mathrm{dd}, J_{2,1}=3.7\right.$ $\left.\mathrm{Hz}, J_{2,3}=9.6 \mathrm{~Hz}, 1 \mathrm{H}, \mathrm{H}-2\right), 3.59$ (dd, $\left.J_{6 \mathrm{~b}, 6 \mathrm{a}}=10.7 \mathrm{~Hz}, 1 \mathrm{H}, \mathrm{H}-6 \mathrm{~b}\right), 1.05$ (s, 9H, $\left.{ }^{t} \mathrm{Bu}\right), 1.05$ (s, $\left.9 \mathrm{H},{ }^{t} \mathrm{Bu}\right), 1.02\left(\mathrm{~s}, 9 \mathrm{H},{ }^{t} \mathrm{Bu}\right), 0.23$ (s, 3H, SiCH$)_{3}, 0.21$ (s, 3H, SiCH 3$), 0.21$ (s, 3H, SiCH $), 0.20$ $\left(\mathrm{s}, 3 \mathrm{H}, \mathrm{SiCH}_{3}\right), 0.15$ (s, 3H, $\left.\mathrm{SiCH}_{3}\right), 0.14\left(\mathrm{~s}, 3 \mathrm{H}, \mathrm{SiCH}_{3}\right) \mathrm{ppm} .{ }^{13} \mathrm{C}\{\mathrm{H}\} \mathrm{NMR}(150 \mathrm{MHz}$, $\left.\mathrm{CDCl}_{3}\right): \delta=139.26,139.22,138.83,138.72,138.30\left(\mathrm{C}_{\text {quat }}, 5 \times \mathrm{C}-\mathrm{Ph}\right), 127.50-128.46(\mathrm{~m}, 25$ $\times$ C-Ph), 104.53 (C-2'), 88.94 (C-1), 83.17 (C-3'), 82.32 (C-3), 81.11 (C-4'), 80.72 (C-5'), 80.60 (C-2), 77.41 (C-4), 75.83, 74.86, 73.27, 72.79, 72.08 (5 × $\left.\mathrm{O}_{\underline{C}} \mathrm{H}_{2} \mathrm{Ph}\right), 71.53(\mathrm{C}-5), 65.96$ (C-1'), 63.57 (C-6'), 61.66 (C-6), 26.14 (triple intensity, 3C- ${ }^{t} \mathrm{Bu}$ ), 26.13 (triple intensity, 3C$\left.{ }^{t} \mathrm{Bu}\right), 26.07$ (triple intensity, $\left.3 \mathrm{C}-{ }^{t} \mathrm{Bu}\right), 18.52,18.45,18.43\left(3 \times \mathrm{C}_{\text {quat, }}{ }^{t} \mathrm{Bu}\right),-4.91,-5.04,-5.10$, 5.24, $-5.24,-5.28\left(6 \times \mathrm{SiCH}_{3}\right)$ ppm. HRMS (ESI-TOF) calcd for $\mathrm{C}_{65} \mathrm{H}_{94} \mathrm{O}_{11} \mathrm{Si} 3 \mathrm{Na}[\mathrm{M}+\mathrm{Na}]^{+}$: 
1157.6002, found: 1157.6008. Anal. calcd for $\mathrm{C}_{67} \mathrm{H}_{94} \mathrm{O}_{12} \mathrm{Si}_{3}\left(\mathrm{C}_{65} \mathrm{H}_{94} \mathrm{O}_{11} \mathrm{Si}_{3}+\mathrm{H}_{2} \mathrm{O}\right)$ : C, 67.83; H, 8.54; found: C, 67.89; H, 8.42.

\section{6. $2,3,3$ ', $4,4^{\prime}$ '-Penta- $O$-benzylsucrose (3)}
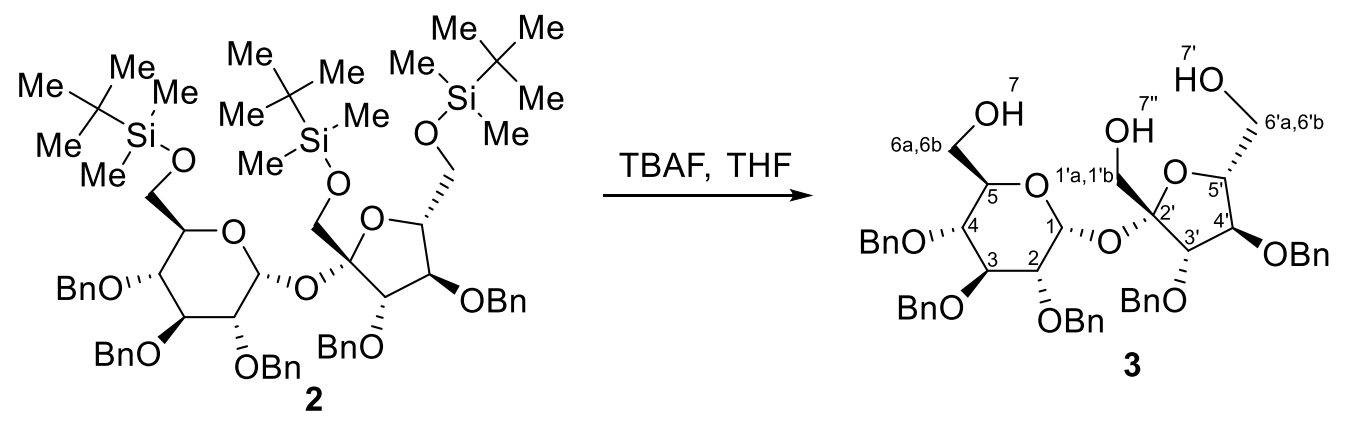

To a solution of $2(7 \mathrm{~g}, 6.16 \mathrm{mmol})$ in THF $(30 \mathrm{~mL})$, a $1 \mathrm{M}$ TBAF solution in THF $(27.7 \mathrm{~mL})$ was added and the mixture was stirred for $24 \mathrm{~h}$ at $60{ }^{\circ} \mathrm{C}$. The solvent was evaporated in vacuo and the residue was purified by column chromatography (hexanes/ethyl acetate $=4: 1$ to $1: 2$ ) to give pure triol $3(3.8 \mathrm{~g}, 78 \%)$ as a colorless oil. $[\alpha]_{\mathrm{D}}=+18.8\left(\mathrm{CHCl}_{3}\right) .{ }^{1} \mathrm{H} \mathrm{NMR}(600 \mathrm{MHz}$, $\left.\mathrm{CDCl}_{3}\right): \delta=7.22-7.42(\mathrm{~m}, 25 \mathrm{H}, 25 \times \mathrm{H}-\mathrm{Ph}), 5.25\left(\mathrm{~d}, J_{1,2}=3.5 \mathrm{~Hz}, 1 \mathrm{H}, \mathrm{H}-1\right), 4.92(\mathrm{~d}, J=11.7$ $\mathrm{Hz}, 1 \mathrm{H}$, benzylic $\mathrm{H}), 4.87(\mathrm{~d}, J=11.0 \mathrm{~Hz}, 1 \mathrm{H}$, benzylic $\mathrm{H}), 4.83(\mathrm{~d}, J=11.5 \mathrm{~Hz}, 1 \mathrm{H}$, benzylic $\mathrm{H}), 4.80(\mathrm{~s}, 2 \mathrm{H}$, benzylic H), $4.69(\mathrm{~d}, J=11.6 \mathrm{~Hz}, 1 \mathrm{H}$, benzylic $\mathrm{H}), 4.64(\mathrm{~d}, J=11.1 \mathrm{~Hz}, 1 \mathrm{H}$, benzylic H), $4.62(\mathrm{~d}, J=11.7 \mathrm{~Hz}, 1 \mathrm{H}$, benzylic $\mathrm{H}), 4.56(\mathrm{~d}, J=11.7 \mathrm{~Hz}, 1 \mathrm{H}$, benzylic $\mathrm{H}), 4.49$ (d, $J=11.6 \mathrm{~Hz}, 1 \mathrm{H}$, benzylic H), 4.11 (m, 1H, H-3'), 4.09 (m, 1H, H-4'), 4.07 (m, 1H, H-5), $4.02\left(\mathrm{dd}, J_{3,2}=9.4 \mathrm{~Hz}, J_{3,4}=9.4 \mathrm{~Hz}, 1 \mathrm{H}, \mathrm{H}-3\right), 3.98\left(\mathrm{dd}, J^{\prime}, 4^{\prime}=9.2 \mathrm{~Hz}, J^{\prime}, 6^{\prime} \mathrm{a}=4.5 \mathrm{~Hz}, 1 \mathrm{H}, \mathrm{H}-\right.$ 5'), $3.89\left(\mathrm{dd}, J_{7^{\prime}, 6^{\prime} \mathrm{a}}=10.9 \mathrm{~Hz}, J_{7^{\prime}, 6^{\prime} \mathrm{b}}=3.3 \mathrm{~Hz}, 1 \mathrm{H}, \mathrm{H}-7^{\prime}\right), 3.83$ (d, $\left.J_{6}{ }^{\prime}, 6^{\prime}{ }^{\prime} \mathrm{b}=11.8 \mathrm{~Hz}, 1 \mathrm{H}, \mathrm{H}-6 \mathrm{a}\right)$, $3.68\left(\mathrm{dd}, J_{6 \mathrm{~b}, 6 \mathrm{a}}=12.0 \mathrm{~Hz}, J_{6 \mathrm{~b}, 5}=3.5 \mathrm{~Hz}, 1 \mathrm{H}, \mathrm{H}-6 \mathrm{~b}\right), 3.61$ (s, 2H, H-1'a, H-1'b), 3.55-3.59 (m, 2H, H-2, H-6'a), 3.49 (dd, $\left.J_{4,5}=9.6 \mathrm{~Hz}, J_{4,3}=9.4 \mathrm{~Hz}, 1 \mathrm{H}, \mathrm{H}-4\right), 3.39$ (dd, $J_{6}{ }^{\prime} \mathrm{b}, 7^{\prime}=10.9 \mathrm{~Hz}$, $J_{6}{ }^{\prime}, 6^{\prime} \mathrm{a}=11.3 \mathrm{~Hz}, 1 \mathrm{H}, \mathrm{H}-6$ 'b), 3.25 (s, 1H, H-7'’), 2.63 (s, 1H, H-7) ppm. ${ }^{13} \mathrm{C}\{\mathrm{H}\}$ NMR (150 $\left.\mathrm{MHz}, \mathrm{CDCl}_{3}\right): \delta=138.37,138.35,138.19,137.81,136.74\left(\mathrm{C}_{\text {quat, }}, 5 \times \mathrm{C}-\mathrm{Ph}\right), 127.72-128.91$ (m, $25 \times \mathrm{C}-\mathrm{Ph}), 106.05$ (C-2'), 91.09 (C-1), 86.35 (C-3'), 82.33 (C-5'), 82.23 (C-4'), 81.99 (C-3), 78.83 (C-2), 77.77 (C-4), 75.69, 75.11, $75.11\left(3 \times \mathrm{OCH}_{2} \mathrm{Ph}\right), 73.87(\mathrm{C}-5), 72.89,72.50$ $\left(2 \times \mathrm{OCH}_{2} \mathrm{Ph}\right), 64.83$ (C-6'), 61.71 (C-6), 60.94 (C-1') ppm. HRMS (ESI-TOF) calcd for $\mathrm{C}_{47} \mathrm{H}_{52} \mathrm{O}_{11} \mathrm{Na}[\mathrm{M}+\mathrm{Na}]^{+}:$815.3407, found: 815.3391. Anal. calcd for $\mathrm{C}_{47} \mathrm{H}_{52} \mathrm{O}_{11}: \mathrm{C}, 71.19 ; \mathrm{H}$, 6.61; found: C, 70.98; H, 6.68.

\subsection{2,3,3',4,4'-Penta- $O$-benzyl-1',6,6'-tri- $O$-(2-tert-butoxy-2-oxoethyl)-sucrose (4)}
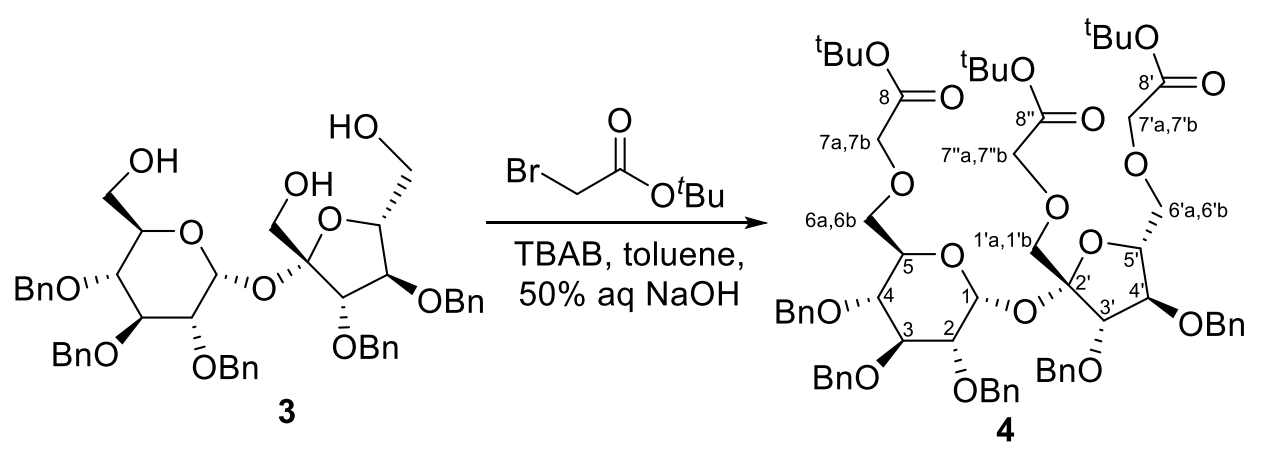
To a solution of triol $3(280 \mathrm{mg}, 0.35 \mathrm{mmol})$ in toluene $(8 \mathrm{~mL}), \mathrm{Bu} 4 \mathrm{NBr}(22.8 \mathrm{mg}, 0.07$ mmol) was added followed by $50 \%$ aqueous $\mathrm{NaOH}(8 \mathrm{~mL})$. Next, tert-butyl bromoacetate $(0.31 \mathrm{~mL}, 2.12 \mathrm{mmol})$ was added and the mixture was vigorously stirred at room temperature for $24 \mathrm{~h}$. The layers were separated and the aqueous one extracted with ether $(3 \times 15 \mathrm{~mL})$. Combined organic solutions were washed with water $(2 \times 10 \mathrm{~mL})$ and brine $(10 \mathrm{~mL})$, dried over $\mathrm{Na}_{2} \mathrm{SO}_{4}$, concentrated, and the residue was purified by flash chromatography (hexanes/ethyl acetate $=90: 10)$ to afford pure product $4(224 \mathrm{mg}, 0.2 \mathrm{mmol}, 56 \%)$ as a yellowish oil. $[\alpha]_{\mathrm{D}}=+33.7\left(\mathrm{CHCl}_{3}\right) .{ }^{1} \mathrm{H}$ NMR $\left(600 \mathrm{MHz}, \mathrm{CDCl}_{3}\right): \delta=7.21-7.37(\mathrm{~m}, 25 \mathrm{H}, 25$ $\times \mathrm{H}-\mathrm{Ph}), 5.69\left(\mathrm{~d}, J_{1,2}=3.6 \mathrm{~Hz}, 1 \mathrm{H}, \mathrm{H}-1\right), 4.91(\mathrm{~d}, J=11.0 \mathrm{~Hz}, 1 \mathrm{H}$, benzylic H), $4.86(\mathrm{~d}, J=$ $10.8 \mathrm{~Hz}, 1 \mathrm{H}$, benzylic $\mathrm{H}), 4.84(\mathrm{~d}, J=11.0 \mathrm{~Hz}, 1 \mathrm{H}$, benzylic $\mathrm{H}), 4.77(\mathrm{~d}, J=11.0 \mathrm{~Hz}, 1 \mathrm{H}$, benzylic $\mathrm{H}), 4.72(\mathrm{~d}, J=11.5 \mathrm{~Hz}, 1 \mathrm{H}$, benzylic $\mathrm{H}), 4.70(\mathrm{~d}, J=12.2 \mathrm{~Hz}, 1 \mathrm{H}$, benzylic $\mathrm{H}), 4.67$ $(\mathrm{d}, J=11.3 \mathrm{~Hz}, 1 \mathrm{H}$, benzylic $\mathrm{H}), 4.62(\mathrm{~d}, J=11.8 \mathrm{~Hz}, 1 \mathrm{H}$, benzylic $\mathrm{H}), 4.59(\mathrm{~d}, J=11.7 \mathrm{~Hz}$, $1 \mathrm{H}$, benzylic H), 4.58 (d, $J=11.7 \mathrm{~Hz}, 1 \mathrm{H}$, benzylic $\mathrm{H}), 4.50\left(\mathrm{~d}, J_{3^{\prime}, 4^{\prime}}=7.4 \mathrm{~Hz}, 1 \mathrm{H}, \mathrm{H}-3^{\prime}\right)$, 4.10-4.14 (m, 2H, H-4', H-5'), 4.06 (m, 1H, H-5), 4.04 (d, J7'a,7'”b = 16.5 Hz, 1H, H-7'’a), $4.03\left(\mathrm{~d}, J_{7}{ }^{\prime} \mathrm{a}, 7^{\prime} \mathrm{b}=16.5 \mathrm{~Hz}, 1 \mathrm{H}, \mathrm{H}-7\right.$ 'a), $3.96\left(\mathrm{~d}, J_{7} \mathrm{~b}, 7^{\prime} \mathrm{a}=16.5 \mathrm{~Hz}, 1 \mathrm{H}, \mathrm{H}-7\right.$ 'b), 3.94 (dd, $J_{3,4}=$ $\left.9.8 \mathrm{~Hz}, J_{3,2}=9.7 \mathrm{~Hz}, 1 \mathrm{H}, \mathrm{H}-3\right), 3.92\left(\mathrm{~d}, J_{7}{ }^{\prime}{ }^{\prime}, 7{ }^{\prime}{ }^{\prime} \mathrm{a}=16.5 \mathrm{~Hz}, 1 \mathrm{H}, \mathrm{H}-7\right.$ ' ’b), 3.90 (d, $J_{1}{ }^{\prime} \mathrm{a}, 1^{\prime} \mathrm{b}=11.1$ Hz, 1H, H-1'a), 3.89 (d, J7a,7b $=16.4$ Hz, 1H, H-7a), 3.85 (d, J Jb,7a $=16.4$ Hz, $1 \mathrm{H}, \mathrm{H}-7 \mathrm{~b}), 3.79$ $\left(\mathrm{dd}, J_{6}{ }^{\prime} \mathrm{a}, 6^{\prime} \mathrm{b}=10.4 \mathrm{~Hz}, J_{6}{ }^{\prime}, 5^{\prime}=6.1 \mathrm{~Hz}, 1 \mathrm{H}, \mathrm{H}-6^{\prime} \mathrm{a}\right), 3.76\left(\mathrm{dd}, J_{6}{ }^{\prime} \mathrm{b}, 6^{\prime} \mathrm{a}=10.4 \mathrm{~Hz}, J_{6}{ }^{\prime} \mathrm{b}, 5^{\prime}=3.6 \mathrm{~Hz}\right.$, $1 \mathrm{H}, \mathrm{H}-6$ 'b), $3.65\left(\mathrm{dd}, J_{4,3}=9.8 \mathrm{~Hz}, J_{4,5}=9.3 \mathrm{~Hz}, 1 \mathrm{H}, \mathrm{H}-4\right), 3.61\left(\mathrm{dd}, J_{6 \mathrm{a}, 6 \mathrm{~b}}=10.8 \mathrm{~Hz}, J_{6 \mathrm{a}, 5}=\right.$ $3.2 \mathrm{~Hz}, 1 \mathrm{H}, \mathrm{H}-6 \mathrm{a}), 3.54\left(\mathrm{dd}, J_{2,3}=9.7 \mathrm{~Hz}, J_{2,1}=3.6 \mathrm{~Hz}, 1 \mathrm{H}, \mathrm{H}-2\right), 3.52\left(\mathrm{~d}, J_{1}{ }^{\prime} \mathrm{b}, 1^{\prime} \mathrm{a}=11.1 \mathrm{~Hz}\right.$, $1 \mathrm{H}, \mathrm{H}-1$ 'b), $3.47\left(\mathrm{dd}, J_{6 \mathrm{~b}, 6 \mathrm{a}}=10.8 \mathrm{~Hz}, J_{6 \mathrm{~b}, 5}=1.7 \mathrm{~Hz}, 1 \mathrm{H}, \mathrm{H}-6 \mathrm{~b}\right), 1.45(\mathrm{~s}, 9 \mathrm{H}, 9 \times-$ $\left.\mathrm{CO}_{2} \mathrm{C}\left(\mathrm{C} \underline{H}_{3}\right)_{3}\right), 1.44\left(\mathrm{~s}, 9 \mathrm{H}, 9 \times-\mathrm{CO}_{2} \mathrm{C}\left(\mathrm{C}_{3}\right)_{3}\right), 1.43\left(\mathrm{~s}, 9 \mathrm{H}, 9 \times-\mathrm{CO}_{2} \mathrm{C}\left(\mathrm{C}_{3}\right)_{3}\right) \mathrm{ppm} .{ }^{13} \mathrm{C}\{\mathrm{H}\}$ NMR (150 MHz, $\left.\mathrm{CDCl}_{3}\right): \delta=169.33,169.29,169.19$ (C-8, C-8', C-8”), 138.96, 138.81, 138.39, 138.39, 138.32 ( $\left.\mathrm{C}_{\text {quat }}, 5 \times \mathrm{C}-\mathrm{Ph}\right), 127.36-128.23(\mathrm{~m}, 25 \times \mathrm{C}-\mathrm{Ph}), 104.46(\mathrm{C}-2$ '), 89.96 (C-1), 83.85 (C-3'), 82.16 (C-5'), 81.91 (C-3), 81.42, 81.31, $81.19\left(3 \times \mathrm{C}_{\text {quat }}, \mathrm{CO}_{2} \underline{C}\left(\mathrm{CH}_{3}\right)_{3}\right)$, 79.59 (C-4'), 79.53 (C-2), 77.29 (C-4), 75.40, 74.70, 73.06 (3 × $\left.\mathrm{O}_{\underline{C}} \mathrm{H}_{2} \mathrm{Ph}\right), 72.62$ (C-6'), 72.41 (C-1'), 72.40, $72.26\left(2 \times \mathrm{OCH}_{2} \mathrm{Ph}\right), 70.54(\mathrm{C}-5), 69.72(\mathrm{C}-6), 69.09$ (C-7'”), 69.04 (C7), 68.97 (C-7'), 28.10, 28.08, 28.08 (triple intensity, $\left.9 \times-\mathrm{CO}_{2} \mathrm{C}\left(\underline{\mathrm{C}} \mathrm{H}_{3}\right)_{3}\right) \mathrm{ppm}$. HRMS (ESITOF) calcd for $\mathrm{C}_{65} \mathrm{H}_{82} \mathrm{O}_{17} \mathrm{Na}[\mathrm{M}+\mathrm{Na}]^{+}: 1157.5450$, found: 1157.5457 . Anal. calcd for $\mathrm{C}_{65} \mathrm{H}_{82} \mathrm{O}_{17}$ : C, 68.76; H, 7.28; found: C, 68.76; H, 7.20.

\subsection{2,3,3',4,4'-Penta-O-benzyl-1',6,6'-tri-O-(2-hydroxyethyl)-sucrose (5)}

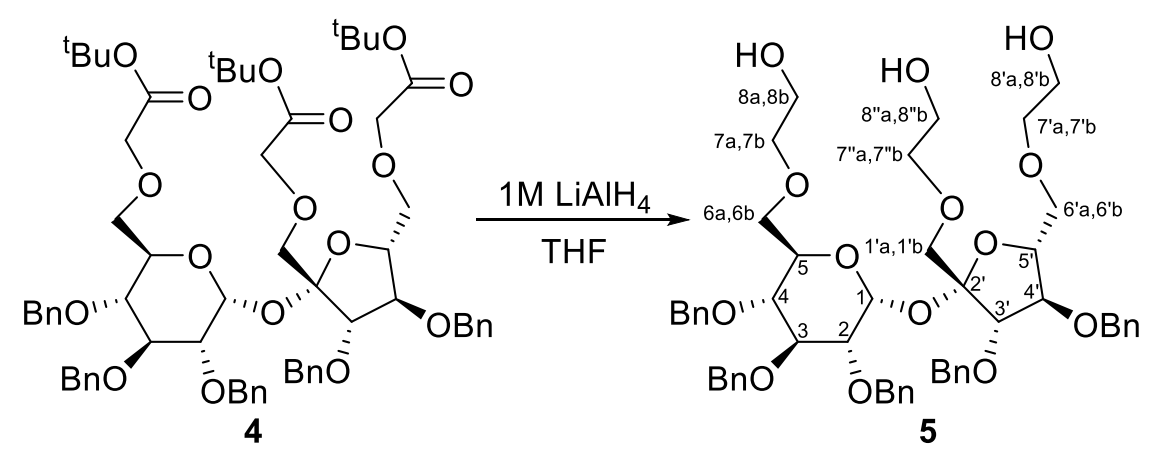

To the cooled to $-78{ }^{\circ} \mathrm{C}$ solution of compound $4(559 \mathrm{mg}, 0.49 \mathrm{mmol})$ in dry THF $(16 \mathrm{~mL})$, $1 \mathrm{M} \mathrm{LiAlH}_{4}$ in THF $(2.95 \mathrm{~mL}, 2.95 \mathrm{mmol})$ was added dropwise, the mixture was allowed to reach room temperature, and stirred additional $20 \mathrm{~min}$ at rt. Excess of hydride was carefully 
decomposed with aqueous saturated $\mathrm{Na}_{2} \mathrm{SO}_{4}(5 \mathrm{~mL})$ and then Celite was added. Next, the resulting residue was filtered and washed with ethyl acetate $(3 \times 10 \mathrm{~mL})$. The organic solution was dried over $\mathrm{Na}_{2} \mathrm{SO}_{4}$, concentrated, and purified by flash chromatography (hexanes/ethyl acetate $=1: 1$ to $1: 4)$ to give pure product $5(408 \mathrm{mg}, 0.44 \mathrm{mmol}, 90 \%)$ as a yellowish oil. $[\alpha]_{\mathrm{D}}=+37.9\left(\mathrm{CHCl}_{3}\right) .{ }^{1} \mathrm{H} \mathrm{NMR}\left(600 \mathrm{MHz}, \mathrm{CDCl}_{3}\right): \delta=7.21-7.38(\mathrm{~m}, 25 \mathrm{H}, 25 \times \mathrm{H}-\mathrm{Ph}), 6.12$ $\left(\mathrm{d}, J_{1,2}=3.9 \mathrm{~Hz}, 1 \mathrm{H}, \mathrm{H}-1\right), 4.98(\mathrm{~d}, J=11.0 \mathrm{~Hz}, 1 \mathrm{H}$, benzylic $\mathrm{H}), 4.82(\mathrm{~d}, J=11.1 \mathrm{~Hz}, 2 \mathrm{H}, 2$ $\times$ benzylic $\mathrm{H}), 4.74(\mathrm{~d}, J=11.2 \mathrm{~Hz}, 2 \mathrm{H}, 2 \times$ benzylic $\mathrm{H}), 4.69\left(\mathrm{dd}, J_{4}, 5^{\prime}=8.6 \mathrm{~Hz}, J_{4}{ }^{\prime}, 3^{\prime}=8.5\right.$ $\mathrm{Hz}, 1 \mathrm{H}, \mathrm{H}-4$ ') 4.66 (s, 2H, $2 \times$ benzylic H), 4.61 (d, $J=11.8 \mathrm{~Hz}, 1 \mathrm{H}$, benzylic H), 4.59 (d, $J$ $=11.1 \mathrm{~Hz}, 1 \mathrm{H}$, benzylic $\mathrm{H}), 4.52(\mathrm{~d}, J=11.2 \mathrm{~Hz}, 1 \mathrm{H}$, benzylic $\mathrm{H}), 4.30\left(\mathrm{~d}, J_{3^{\prime}, 4^{\prime}}=8.5 \mathrm{~Hz}, 1 \mathrm{H}\right.$, H-3'), 4.15 (m, 1H, H-5), 3.92 (dd, $\left.J_{3,2}=9.3 \mathrm{~Hz}, J_{3,4}=9.2 \mathrm{~Hz}, 1 \mathrm{H}, \mathrm{H}-3\right), 3.84-3.94$ (m, 2H, H-5', H-6'a), 3.62-3.71 (m, 5H, H-4, H-1'a, H-8''a, H-8' 'b, H-8a), 3.61 (m, 2H, H-7' 'a, H7 ' 'b), 3.55 (d, $J_{1}$ 'b, '’a $=10.8 \mathrm{~Hz}, 1 \mathrm{H}, \mathrm{H}-1$ 'b), 3.52 (m, 1H, H-8b), 3.46 (dd, $J_{2,3}=9.6 \mathrm{~Hz}, J_{2,1}=$ $3.9 \mathrm{~Hz}, 1 \mathrm{H}, \mathrm{H}-2$ ), 3.42 (m, 1H, H-8'b), 3.38 (m, 1H, H-6'b), 3.24-3.35 (m, 7H, H-8'b, H-6a, H-6b, H-7a, H-7b, H-7'a, H-7’b) ppm. ${ }^{13} \mathrm{C}\{\mathrm{H}\} \mathrm{NMR}\left(150 \mathrm{MHz}, \mathrm{CDCl}_{3}\right)$ : $\delta=139.08,138.68$, 138.02, 137.95, 137.65 (C quat, $5 \times \mathrm{C}-\mathrm{Ph}), 127.34-128.54(\mathrm{~m}, 25 \times \mathrm{C}-\mathrm{Ph}), 103.57\left(\mathrm{C}-2{ }^{\prime}\right), 88.05$ (C-1), 83.74 (C-3'), 81.92 (C-3), 79.01 (C-5'), 78.92 (C-4'), 78.76 (C-2), 77.03 (C-4), 75.27,

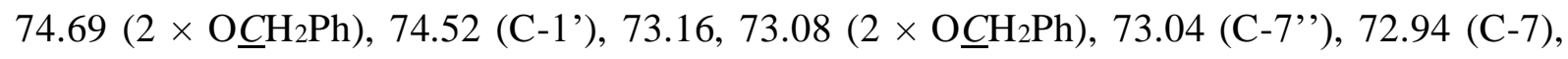
72.25 (C-7'), $72.02\left(\mathrm{O} \underline{\mathrm{C}} \mathrm{H}_{2} \mathrm{Ph}\right), 70.61$ (C-5), 68.44 (C-6'), 68.40 (C-6), 61.62, 61.61, 61.57 (C-8, C-8', C-8'') ppm. HRMS (ESI-TOF) calcd for $\mathrm{C}_{53} \mathrm{H}_{64} \mathrm{O}_{14} \mathrm{Na}[\mathrm{M}+\mathrm{Na}]^{+}: 947.4194$, found: 947.4183. Anal. calcd for $\mathrm{C}_{53} \mathrm{H}_{64} \mathrm{O}_{14}$ : C, 68.81; $\mathrm{H}, 6.97$; found: $\mathrm{C}, 68.75 ; \mathrm{H}, 6.95$.

\subsection{2,3,3',4,4'-Penta- $O$-benzyl-1',6,6'-tri-O-(2-iodoethyl)-sucrose (6)}

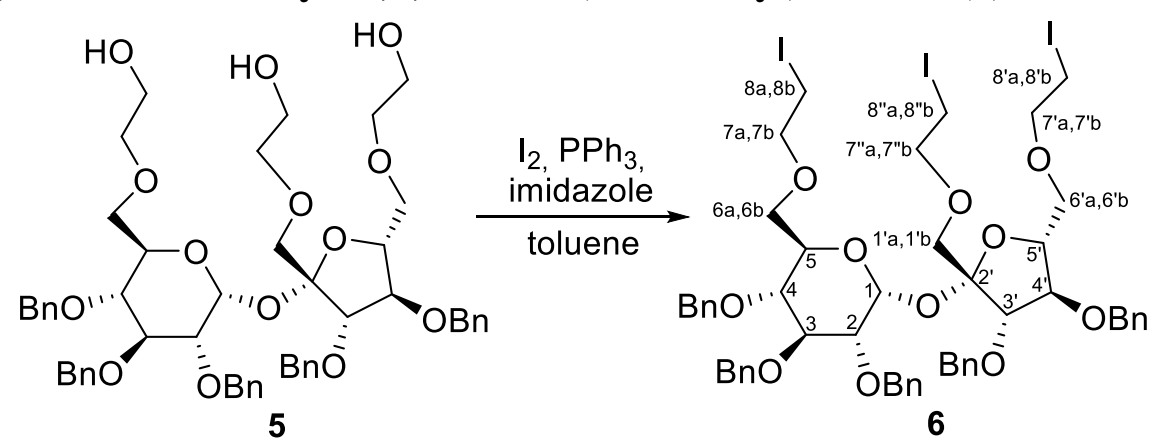

To a solution of triol 5 (702 mg, $0.759 \mathrm{mmol})$ in toluene $(25 \mathrm{~mL}), \mathrm{PPh}_{3}(716 \mathrm{mg}, 2.73 \mathrm{mmol})$ was added, the mixture was boiled under reflux for $15 \mathrm{~min}$, and then cooled to $50{ }^{\circ} \mathrm{C}$. Imidazole ( $310 \mathrm{mg}, 4.55 \mathrm{mmol}$ ) was added, the mixture was allowed to reach room temperature, and the solution of iodine $(693 \mathrm{mg}, 2.73 \mathrm{mmol})$ in toluene $(20 \mathrm{~mL})$ was added dropwise during $20 \mathrm{~min}$. After another $20 \mathrm{~min}$, the excess of iodine was decomposed with saturated $\mathrm{Na}_{2} \mathrm{~S}_{2} \mathrm{O}_{3}(5 \mathrm{~mL})$. Water $(20 \mathrm{~mL})$ was added, the phases were separated, the aqueous one was extracted with ethyl acetate $(2 \times 20 \mathrm{~mL})$ and combined organic phases were washed with water $(30 \mathrm{~mL})$, brine $(30 \mathrm{~mL})$, dried over $\mathrm{Na}_{2} \mathrm{SO}_{4}$ and concentrated. The resulting residue was purified by flash chromatography (hexanes/ethyl acetate $=9: 1$ ) to afford compound $6(810 \mathrm{mg}, 0.64 \mathrm{mmol}, 85 \%)$ as a colorless oil. $[\alpha]_{\mathrm{D}}=+33.8\left(\mathrm{CHCl}_{3}\right) .{ }^{1} \mathrm{H}$ NMR (600 MHz, $\left.\mathrm{CDCl}_{3}\right): \delta=7.24-7.37(\mathrm{~m}, 25 \mathrm{H}, 25 \times \mathrm{H}-\mathrm{Ph}), 5.65\left(\mathrm{~d}, J_{1,2}=3.5 \mathrm{~Hz}, 1 \mathrm{H}, \mathrm{H}-1\right), 4.94$ $(\mathrm{d}, J=10.9 \mathrm{~Hz}, 1 \mathrm{H}$, benzylic $\mathrm{H}), 4.90(\mathrm{~d}, J=11.1 \mathrm{~Hz}, 1 \mathrm{H}$, benzylic $\mathrm{H}), 4.80(\mathrm{~d}, J=10.9 \mathrm{~Hz}$, $1 \mathrm{H}$, benzylic $\mathrm{H}), 4.79(\mathrm{~d}, J=11.4 \mathrm{~Hz}, 1 \mathrm{H}$, benzylic $\mathrm{H}), 4.68(\mathrm{~d}, J=11.7 \mathrm{~Hz}, 1 \mathrm{H}$, benzylic $\mathrm{H})$, $4.67(\mathrm{~d}, J=11.5 \mathrm{~Hz}, 1 \mathrm{H}$, benzylic H), $4.66(\mathrm{~d}, J=11.8 \mathrm{~Hz}, 1 \mathrm{H}$, benzylic $\mathrm{H}), 4.64(\mathrm{~d}, J=11.5$ 
$\mathrm{Hz}, 1 \mathrm{H}$, benzylic $\mathrm{H}), 4.63(\mathrm{~d}, J=11.8 \mathrm{~Hz}, 1 \mathrm{H}$, benzylic $\mathrm{H}), 4.59(\mathrm{~d}, J=11.8 \mathrm{~Hz}, 1 \mathrm{H}$, benzylic H), $4.45\left(\mathrm{~d}, J_{3^{\prime}, 4^{\prime}}=7.3 \mathrm{~Hz}, 1 \mathrm{H}, \mathrm{H}-3^{\prime}\right), 4.12\left(\mathrm{dd}, J_{4^{\prime}, 3^{\prime}}=7.3 \mathrm{~Hz}, J_{4^{\prime}, 5^{\prime}}=7.3 \mathrm{~Hz}, 1 \mathrm{H}, \mathrm{H}-4^{\prime}\right), 4.07$ (m, 1H, H-5'), 4.04 (m, 1H, H-5), 3.95 (dd, J3,2 = 9.4 Hz, J3,4 = 9.3 Hz, 1H, H-3), 3.65-3.73 (m, 7H, H-7a, H-7'a, H-7''a, H-7' 'b, H-6'a, H-6'b, H-1'a), 3.64 (m, 1H, H-4), 3.57 (m, 1H, H-6a), 3.51-3.56 (m, 3H, H-7b, H-7’b, H-2), 3.46 (d, J'’b, 'a = 11.0 Hz, 1H, H-1'b), 3.39 (dd, $\left.J_{6 \mathrm{~b}, 6 \mathrm{a}}=10.7 \mathrm{~Hz}, J_{6 \mathrm{~b}, 5}=1.4 \mathrm{~Hz}, 1 \mathrm{H}, \mathrm{H}-6 \mathrm{~b}\right), 3.08-3.22$ (m, 6H, H-8a, H-8b, H-8'a, H-8'b, H8 "'a, H-8"'b) ppm. ${ }^{13} \mathrm{C}\{\mathrm{H}\}$ NMR (150 MHz, $\left.\mathrm{CDCl}_{3}\right): \delta=138.76,138.59,138.23,138.16$, $138.13\left(\mathrm{C}_{\text {quat }}, 5 \times \mathrm{C}-\mathrm{Ph}\right), 127.53-128.38(\mathrm{~m}, 25 \times \mathrm{C}-\mathrm{Ph}), 104.38\left(\mathrm{C}-2^{\prime}\right), 90.10(\mathrm{C}-1), 83.67$ (C-3'), 82.18 (C-4'), 81.95 (C-3), 79.83 (C-2), 79.59 (C-5'), 77.39 (C-4), 75.54, 74.94, 73.13, 72.67, $72.53\left(5 \times \mathrm{OCH}_{2} \mathrm{Ph}\right), 72.16\left(\mathrm{C}^{\prime} 7^{\prime}\right), 72.06$ (C-7), 71.95 (C-7’'), 71.81 (C-6'), 71.67 (C1'), 70.63 (C-5), 69.25 (C-6), 2.82, 2.80, 2.73 (C-8, C-8', C-8'’) ppm. HRMS (ESI-TOF) calcd for $\mathrm{C}_{53} \mathrm{H}_{61} \mathrm{O}_{11} \mathrm{I}_{3} \mathrm{Na}\left[\mathrm{M}+\mathrm{Na}^{+}:\right.$1277.1246, found: 1277.1244. Anal. calcd for $\mathrm{C}_{53} \mathrm{H}_{61} \mathrm{O}_{11} \mathrm{I}_{3}$ : C, 50.73; H, 4.90; found: C, 50.72; H, 5.01.

\subsection{General procedure for the synthesis of compounds $M-8$ and $P-8$}

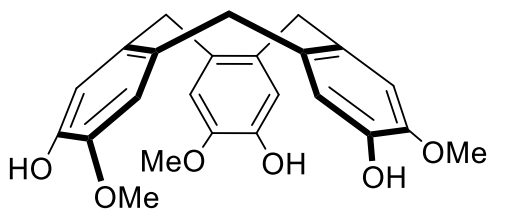

(士) 7

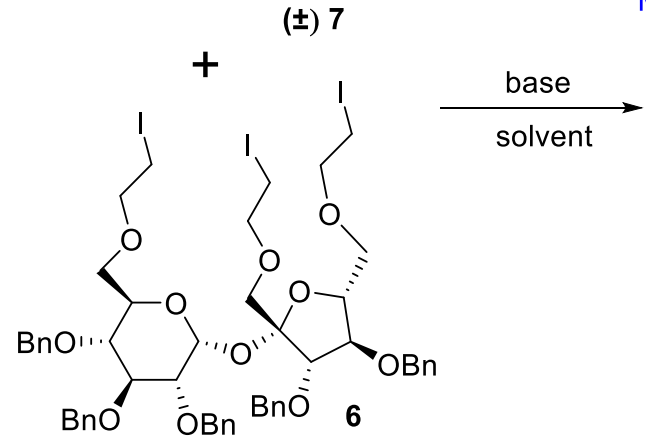

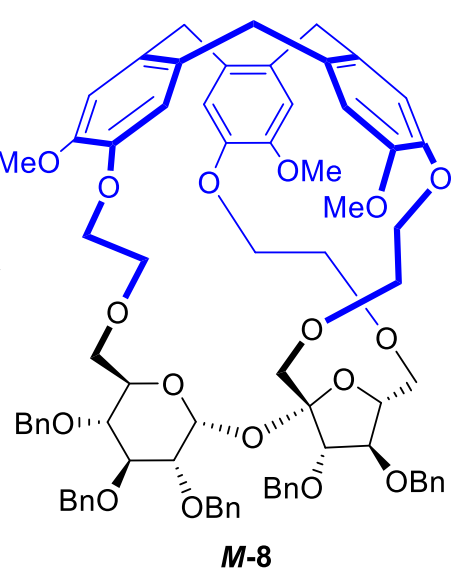

M-8

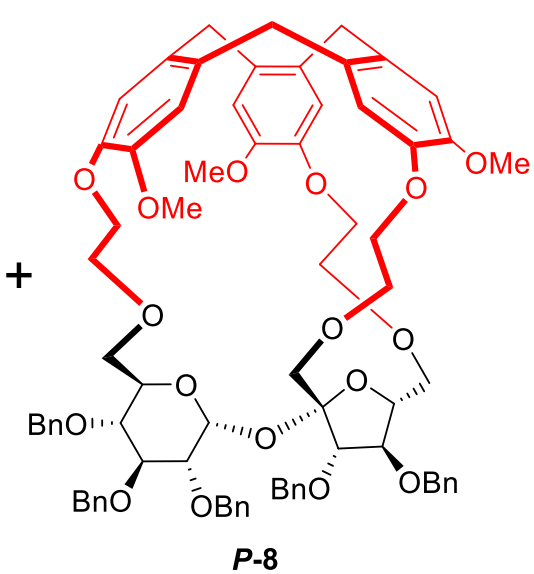

To a solution $(0.002 \mathrm{M}, 160 \mathrm{~mL}$ or $0.001 \mathrm{M}, 320 \mathrm{~mL})$ of $\boldsymbol{r a c}-7(130 \mathrm{mg}, 0.319 \mathrm{mmol})$ in dry solvent (acetone/acetonitrile/propionitrile/DMF), cesium carbonate (623 mg, $1.91 \mathrm{mmol}$ ) was added and the mixture was stirred at room temperature for 30 min under an argon atmosphere. The solution of compound $6(400 \mathrm{mg}, 0.319 \mathrm{mmol})$ in appropriate dry solvent $(10 \mathrm{~mL})$ was added dropwise during $40 \mathrm{~min}$, the mixture was stirred at required temperature for $48-96 \mathrm{~h}$ (for details see Table 1 of the main text). After cooling to rt., the mixture was filtered through Celite and the solvents were removed under vacuum. The residue was dissolved in $\mathrm{CH}_{2} \mathrm{Cl}_{2}$ $(20 \mathrm{~mL})$ and washed with water $(2 \times 10 \mathrm{~mL})$. The aqueous phases were extracted with $\mathrm{CH}_{2} \mathrm{Cl}_{2}$ $(2 \times 20 \mathrm{~mL})$ and the combined organic solutions were washed with brine $(20 \mathrm{~mL})$, dried over $\mathrm{Na}_{2} \mathrm{SO}_{4}$, and concentrated. The resulting residue was purified by flash chromatography (hexanes/ethyl acetate $=4: 1$ to 3:1) to afford pure compounds $\boldsymbol{M - 8}$ and $\boldsymbol{P - 8}$ as colorless solids. 


\subsubsection{Characterization of compound $M-8$}

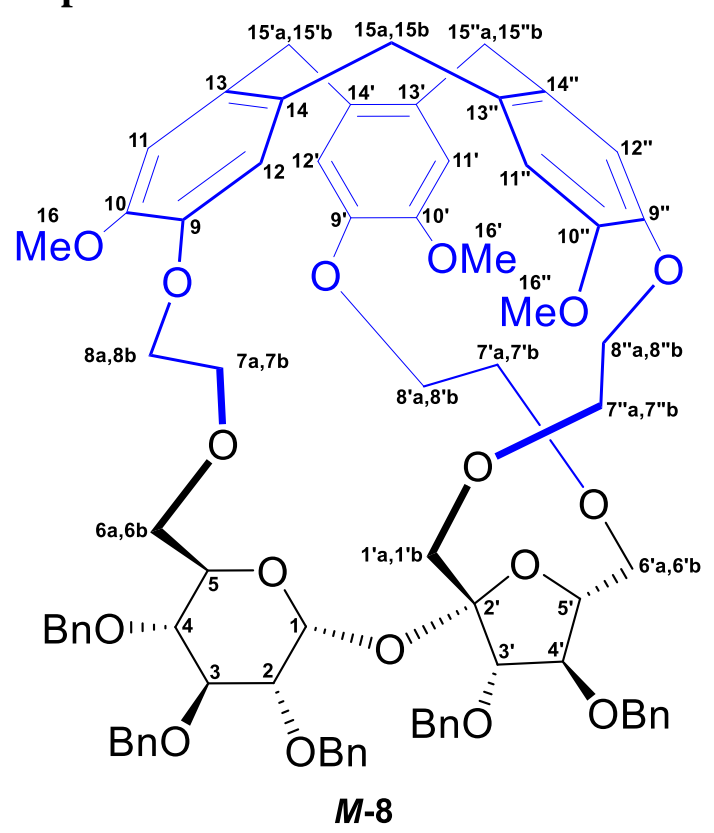

$[\alpha]_{\mathrm{D}}=-60.7\left(\mathrm{CHCl}_{3}\right) .{ }^{1} \mathrm{H}$ NMR $\left(600 \mathrm{MHz}, \mathrm{CD}_{3} \mathrm{CN}\right): \delta=7.16-7.43(\mathrm{~m}, 25 \mathrm{H}, 25 \times \mathrm{H}-\mathrm{Ph})$, 7.024 (s, 1H, H-12'), 7.009 (s, 1H, H-11), 6.972 (s, 1H, H-12), 6.963 (s, 1H, H-11'), 6.910 (s, 1H, H-11"), 6.837 (s, 1H, H-12”), 5.26 (d, J1,2 = 3.1 Hz, 1H, H-1), 4.90 (d, J= $11.3 \mathrm{~Hz}, 1 \mathrm{H}$, benzylic H), 4.75 (d, $J_{15}$ ”b,15”a $=13.3 \mathrm{~Hz}, 1 \mathrm{H}, \mathrm{H}-15 ”$ ”), $4.74(\mathrm{~d}, J=11.3 \mathrm{~Hz}, 1 \mathrm{H}$, benzylic H), $4.72(\mathrm{~d}, J=11.0 \mathrm{~Hz}, 1 \mathrm{H}$, benzylic H), 4.72 (d, 1H, H-15'b), 4.69 (d, $J=11.2 \mathrm{~Hz}, 1 \mathrm{H}$, benzylic H), $4.68\left(\mathrm{~d}, J_{15 \mathrm{~b}, 15 \mathrm{a}}=13.7 \mathrm{~Hz}, 1 \mathrm{H}, \mathrm{H}-15 \mathrm{~b}\right), 4.65(\mathrm{~d}, J=11.8 \mathrm{~Hz}, 1 \mathrm{H}$, benzylic H), $4.62(\mathrm{~d}, J=11.6 \mathrm{~Hz}, 1 \mathrm{H}$, benzylic H), $4.59(\mathrm{~d}, J=11.0 \mathrm{~Hz}, 1 \mathrm{H}$, benzylic $\mathrm{H}), 4.54(\mathrm{~d}, J=11.2$ $\mathrm{Hz}, 1 \mathrm{H}$, benzylic $\mathrm{H}), 4.49(\mathrm{~d}, J=11.8 \mathrm{~Hz}, 1 \mathrm{H}$, benzylic $\mathrm{H}), 4.45(\mathrm{~d}, J=11.6 \mathrm{~Hz}, 1 \mathrm{H}$, benzylic

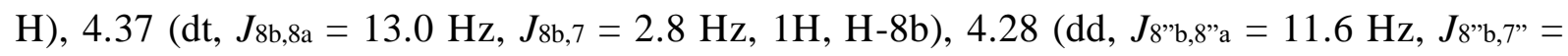
$7.0 \mathrm{~Hz}, 1 \mathrm{H}, \mathrm{H}-8$ '”), 4.23 (m, 1H, H-8'b), 4.19 (m, 1H, H-8'a), 4.16 (m, 1H, H-8”a), 4.12 (m,

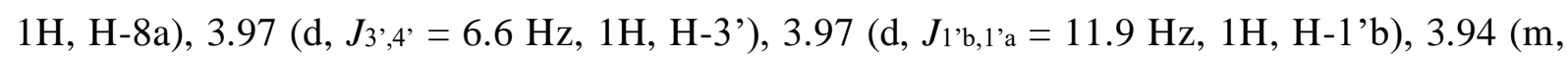
1H, H-7”'b), 3.79 (s, 3H, $3 \times \mathrm{H}-16$ "), 3.77 (dd, $J_{6 \mathrm{a}, 6 \mathrm{~b}}=13.0 \mathrm{~Hz}, J_{6 \mathrm{a}, 5}=2.4 \mathrm{~Hz}, 1 \mathrm{H}, \mathrm{H}-6 \mathrm{~b}$ ), 3.74 (s, $3 \mathrm{H}, 3 \times \mathrm{H}-16), 3.74\left(\mathrm{dd}, J_{7 \mathrm{~b}, 7 \mathrm{a}}=13.3 \mathrm{~Hz}, J_{7 \mathrm{~b}, 8}=2.4 \mathrm{~Hz}, 1 \mathrm{H}, \mathrm{H}-7 \mathrm{~b}\right), 3.72$ (s, 3H, $3 \times \mathrm{H}-16$ '), 3.72 (m, 1H, H-7’'a), 3.65 (m, 1H, H-4'), 3.65 (m, 1H, H-3), 3.56 (m, 1H, H-7a), 3.53 (m, 1H, H-4), 3.52 (d, J15”a,15”b = 13.3 Hz, 1H, H-15”a), 3.50 (d, J15'a,15’b = 13.7 Hz ,1H, H-15'a), 3.49 $\left(\mathrm{d}, J_{15 \mathrm{a}, 15 \mathrm{~b}}=13.7 \mathrm{~Hz}, 1 \mathrm{H}, \mathrm{H}-15 \mathrm{a}\right), 3.42\left(\mathrm{~d}, J_{1}^{\prime} \mathrm{a}, 1^{\prime} \mathrm{b}=11.9 \mathrm{~Hz}, 1 \mathrm{H}, \mathrm{H}-1\right.$ 'a), 3.37 (dd, $J_{2,3}=9.7$ $\left.\mathrm{Hz}, J_{2,1}=3.1 \mathrm{~Hz}, 1 \mathrm{H}, \mathrm{H}-2\right), 3.36$ (m, 1H, H-5'), 3.34 (m, 1H, H-5), 3.28 (m, 1H, H-7’b), 2.96 $\left(\mathrm{dt}, J_{7}{ }^{\prime}, 7^{\prime} \mathrm{b}=10.8 \mathrm{~Hz}, J_{7}{ }^{\mathrm{a}, 8}=3.5 \mathrm{~Hz}, 1 \mathrm{H}, \mathrm{H}-7\right.$ 'a), $2.89\left(\mathrm{dd}, J_{6 \mathrm{a}, 6 \mathrm{~b}}=13.0 \mathrm{~Hz}, J_{6 \mathrm{a}, 5}=0.8 \mathrm{~Hz}, 1 \mathrm{H}\right.$,

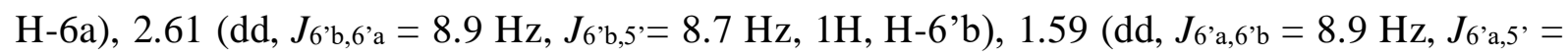
$5.0 \mathrm{~Hz}, 1 \mathrm{H}, \mathrm{H}-6$ 'a) ppm. ${ }^{13} \mathrm{C}\{\mathrm{H}\}$ NMR $\left(150 \mathrm{MHz}, \mathrm{CD}_{3} \mathrm{CN}\right): \delta=150.55(\mathrm{C}-10$ ') $149.75(\mathrm{C}-$ 10), 148.06 (C-10”), 146.77 (C-9”), 145.60 (C-9), 145.03 (C-9'), 140.15, 140.00, 140.01, 139.87, 139.81 (C quat, $5 \times \mathrm{C}-\mathrm{Ph}), 135.15$ (C-13'), 134.06 (C-13), 133.30 (C-14), 132.95 (C14”), 131.91 (C-13”), 131.74 (C-14'), 128.21-129.33 (m, $25 \times \mathrm{C}-\mathrm{Ph}), 121.82$ (C-12'), 117.41 (C-12), 114.96 (C-11), 114.14 (C-11'), 113.62 (C-12”), 113.04 (C-11”), 105.34 (C-2'), 91.64 (C-1), 86.59 (C-4'), 85.06 (C-3'), 82.22 (C-3), 81.81 (C-2), 80.54 (C-5'), 78.08 (C-4), 75.93, $75.34,73.83\left(3 \times \mathrm{OC}_{2} \mathrm{Ph}\right), 73.67(\mathrm{C}-6)$ ') $73.22(\mathrm{C}-5), 72.47,72.39\left(2 \times \mathrm{O}_{\mathrm{C}} \mathrm{H}_{2} \mathrm{Ph}\right), 70.83(\mathrm{C}-$ 1'), 70.76 (C-6), 70.65 (C-8”), 69.91 (C-7), 69.69 (C-8'), 69.21 (C-7’), 68.94 (C-8), 67.57 (C7'), 56.89 (C-16), 56.39 (C-16'), 56.24 (C-16”), 36.32 (C-15”), 36.18 (C-15), 35.87 (C-15') 
ppm. HRMS (ESI-TOF) calcd for $\mathrm{C}_{77} \mathrm{H}_{82} \mathrm{O}_{17} \mathrm{Na}[\mathrm{M}+\mathrm{Na}]^{+}:$1301.5450, found: 1301.5447. Anal. calcd for $\mathrm{C}_{77} \mathrm{H}_{82} \mathrm{O}_{17}$ : C, 72.28; H, 6.46; found: C, 72.26; H, 6.67.

\subsubsection{Characterization of compound $P-8$}

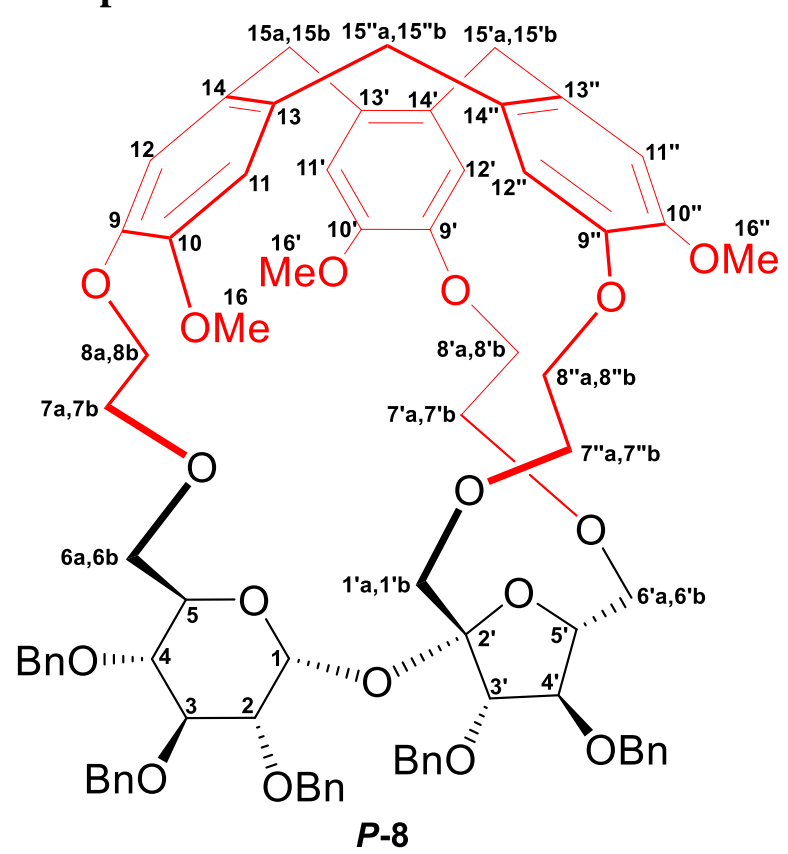

$[\alpha]_{\mathrm{D}}=+101.4\left(\mathrm{CHCl}_{3}\right) .{ }^{1} \mathrm{H} \mathrm{NMR}\left(600 \mathrm{MHz}, \mathrm{CD}_{3} \mathrm{CN}\right): \delta=7.20-7.35(\mathrm{~m}, 25 \mathrm{H}, 25 \times \mathrm{H}-\mathrm{Ph})$, 7.004 (s, 1H, H-11), 6.971 (s, 1H, H-12'), 6.919 (s, 1H, H-11”), 6.915 (s, 1H, H-11'), 6.902 (s, 1H, H-12), 6.896 (s, 1H, H-12”), 4.93 (d, $\left.J_{1,2}=3.5 \mathrm{~Hz}, 1 \mathrm{H}, \mathrm{H}-1\right), 4.75$ (d, $J_{15 \mathrm{~b}, 15 \mathrm{a}}=13.3$ $\mathrm{Hz}, 1 \mathrm{H}, \mathrm{H}-15 \mathrm{~b}), 4.74$ (d, $J=11.3 \mathrm{~Hz}, 1 \mathrm{H}$, benzylic $\mathrm{H}), 4.72(\mathrm{~d}, J=11.3 \mathrm{~Hz}, 1 \mathrm{H}$, benzylic $\mathrm{H})$, $4.70\left(\mathrm{~m}, 1 \mathrm{H}, \mathrm{H}-15^{\prime} \mathrm{b}\right), 4.66(\mathrm{~m}, 2 \mathrm{H}, 2 \times$ benzylic $\mathrm{H}), 4.65(\mathrm{~m}, 1 \mathrm{H}$, benzylic $\mathrm{H}), 4.65(\mathrm{~d}, J=$ $11.3 \mathrm{~Hz}, 1 \mathrm{H}$, benzylic H), 4.65 (d, $J_{15}$ ”b,15”a $\left.=13.5 \mathrm{~Hz}, 1 \mathrm{H}, \mathrm{H}-15 ” b\right), 4.62$ (d, J=11.7 Hz, 1H, benzylic H), 4.55 (d, $J=11.3 \mathrm{~Hz}, 1 \mathrm{H}$, benzylic $\mathrm{H}), 4.52(\mathrm{~d}, J=11.7 \mathrm{~Hz}, 1 \mathrm{H}$, benzylic $\mathrm{H}), 4.47$ $\left(\mathrm{d}, J=11.3 \mathrm{~Hz}, 1 \mathrm{H}\right.$, benzylic H), $4.28\left(\mathrm{ddd}, J_{8} " \mathrm{~b}, 8{ }^{\prime a}=12.3 \mathrm{~Hz}, J_{8}{ }^{\prime \prime}, 7{ }^{\prime} \mathrm{a}=6.1 \mathrm{~Hz}, J_{8}{ }^{\prime} \mathrm{b}, 7{ }^{\prime} \mathrm{b}=1.5\right.$ Hz, 1H, H-8'b), 4.22 (m, 2H, H-8a, H-8b), 4.12 (d, J J', ' = 7.5 Hz, 1H, H-3'), 4.09 (ddd,

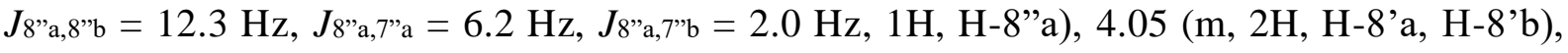
3.772 (s, $3 \mathrm{H}, 3 \times \mathrm{H}-16$ ), 3.75 (m, 1H, H-7'’b), 3.71 (dd, $J^{\prime}{ }^{\prime}{ }^{\prime}=7.6 \mathrm{~Hz}, J^{\prime}{ }^{\prime}{ }^{\prime}=7.5 \mathrm{~Hz}, 1 \mathrm{H}, \mathrm{H}-$ 4'), 3.678 (s, 3H, $3 \times \mathrm{H}-16$ '), 3.653 (s, 3H, $3 \times \mathrm{H}-16$ "), 3.646 (m, 1H, H-3), 3.62 (m, 1H, H7”a), 3.56 (m, 1H, H-7b), 3.49 (d, J15a,15b = $13.3 \mathrm{~Hz}, 1 \mathrm{H}, \mathrm{H}-15 \mathrm{a}), 3.48$ (d, $J_{15}$ ”, 15 ”b $=13.5 \mathrm{~Hz}$, 1H, H-15”a), 3.47 (m, 1H, H-7a), 3.47 (d, J'b,l'a = 11.6 Hz, 1H, H-1 'b), 3.46 (m, 1H, H-15'a), $3.456(\mathrm{~m}, 1 \mathrm{H}, \mathrm{H}-4), 3.436(\mathrm{~m}, 1 \mathrm{H}, \mathrm{H}-5), 3.42(\mathrm{~m}, 1 \mathrm{H}, \mathrm{H}-6 \mathrm{~b}), 3.354\left(\mathrm{dd}, J_{2,3}=9.6 \mathrm{~Hz}, J_{2,1}=\right.$ $3.5 \mathrm{~Hz}, 1 \mathrm{H}, \mathrm{H}-2), 3.32$ (m, 1H, H-7'b), 3.21 (d, $J_{1}^{\prime} \mathrm{a}, 1^{\prime} \mathrm{b}=11.6 \mathrm{~Hz}, 1 \mathrm{H}, \mathrm{H}-1$ 'a), 3.20 (m, 1H, H-

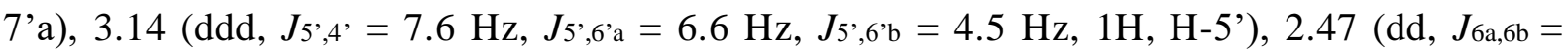
$\left.11.6 \mathrm{~Hz}, J_{6 \mathrm{a}, 5}=1.6 \mathrm{~Hz}, 1 \mathrm{H}, \mathrm{H}-6 \mathrm{a}\right), 2.29\left(\mathrm{dd}, J_{6}{ }^{\prime}, 6^{\prime}{ }^{\circ} \mathrm{b}=11.5 \mathrm{~Hz}, J_{6}{ }^{\prime}, 5^{\prime}=6.6 \mathrm{~Hz}, 1 \mathrm{H}, \mathrm{H}-6^{\prime} \mathrm{a}\right)$, $2.02\left(\mathrm{dd}, J_{6}{ }^{\prime} \mathrm{b}, 6^{\prime} \mathrm{a}=11.5 \mathrm{~Hz}, J_{6}{ }^{\prime} \mathrm{b}, 5{ }^{\prime}=4.5 \mathrm{~Hz}, 1 \mathrm{H}, \mathrm{H}-6\right.$ 'b $)$ ppm. ${ }^{13} \mathrm{C}\{\mathrm{H}\}$ NMR $(150 \mathrm{MHz}$, $\left.\mathrm{CD}_{3} \mathrm{CN}\right): \delta=150.39$ (C-10'), 150.23 (C-10), 148.33 (C-10”), 146.47 (C-9”), 145.75 (C-9), 145.45 (C-9'), 140.15, 139.99, 139.92, 139.75, 139.68 (C quat, $5 \times \mathrm{C}-\mathrm{Ph}), 135.48$ (C-13'), 134.01 (C-14), 133.88 (C-14”), 133.40 (C-13”), 132.47, 132.42 (C-13, C-14'), 128.31-129.29 (m, $25 \times \mathrm{C}-\mathrm{Ph}), 122.32$ (C-12'), 119.49 (C-12), 115.98 (C-11), 115.41 (C-12”), 113.92 (C11'), 113.85 (C-11'), 104.22 (C-2'), 92.04 (C-1), 84.90 (C-3'), 84.61 (C-4'), 82.51 (C-3), 81.19 (C-2), 78.62 (C-5'), $78.17(\mathrm{C}-4), 75.83,75.16,73.90,72.87$ (4 × $\left.\underline{\mathrm{O}}^{\mathrm{H}} \mathrm{H}_{2} \mathrm{Ph}\right), 72.74(\mathrm{C}-$ 
6'), $72.71\left(\mathrm{O}_{\mathrm{CH}} \mathrm{H}_{2} \mathrm{Ph}\right), 71.83$ (C-5), 70.63 (C-7'), 70.39 (C-1'), 70.17 (C-6), 69.96 (C-8'), 69.89 (C-7”), 69.46 (C-7), 68.09 (C-8), 66.94 (C-8”), 57.59 (C-16), 56.40 (C-16'), 56.11 (C16”), 36.33 (C-15”), 36.20 (C-15), 35.95 (C-15') ppm. HRMS (ESI-TOF) calcd for $\mathrm{C}_{77} \mathrm{H}_{82} \mathrm{O}_{17} \mathrm{Na}[\mathrm{M}+\mathrm{Na}]^{+}:$1301.5450, found: 1301.5426 . Anal. calcd for $\mathrm{C}_{77} \mathrm{H}_{82} \mathrm{O}_{17}: \mathrm{C}, 72.28$; $\mathrm{H}, 6.46$; found: $\mathrm{C}, 72.30 ; \mathrm{H}, 6.53$.

\subsection{Compound $M-9$}
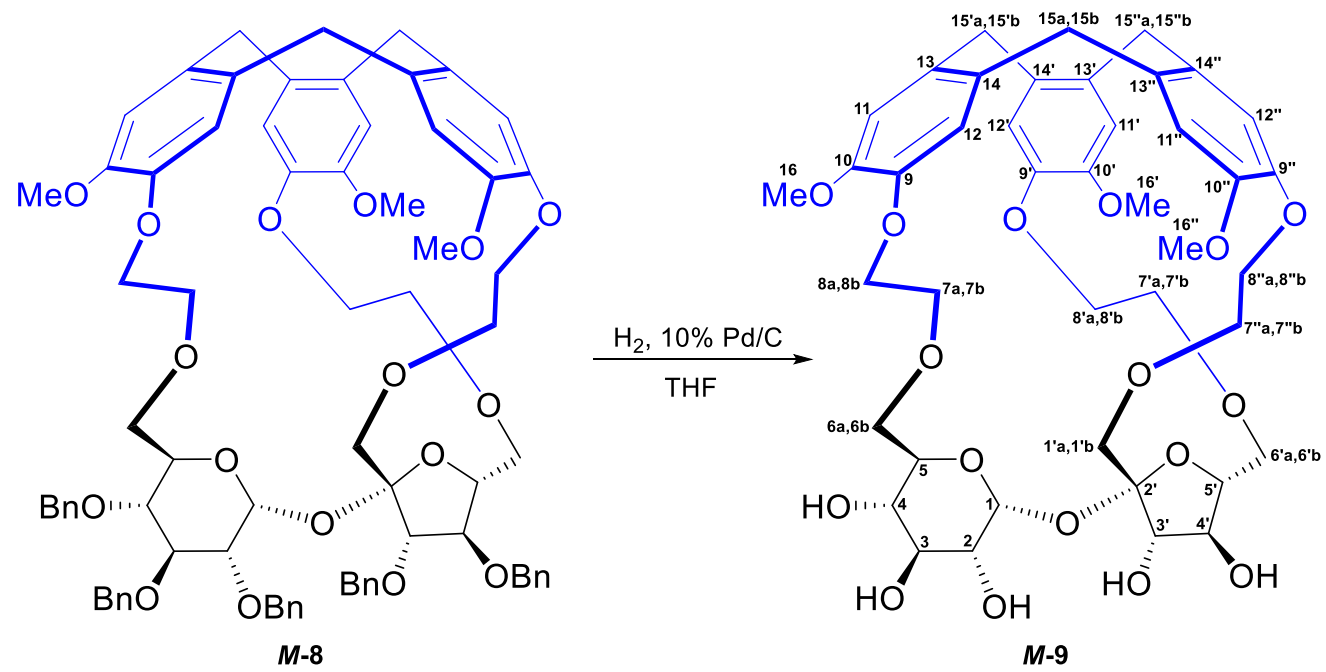

To a solution of compound $\boldsymbol{M - 8}(51 \mathrm{mg}, 0.04 \mathrm{mmol})$ in THF (4 mL), 10\% Pd/C (10.6 mg, $0.01 \mathrm{mmol}$ ) was added and the mixture was stirred under a hydrogen atmosphere in an autoclave for $24 \mathrm{~h}$ at room temperature. The mixture was filtered through Celite, washed with THF $(20 \mathrm{~mL})$, and concentrated under reduced pressure. The pure product $\boldsymbol{M} \mathbf{- 9}(32.5 \mathrm{mg}$, $98 \%)$ was obtained as a white solid without further purification. $[\alpha]_{\mathrm{D}}=-43.7\left(\mathrm{CHCl}_{3}\right) .{ }^{1} \mathrm{H}$ NMR (500 MHz, CD $3 \mathrm{OD}$ ): $\delta=7.07$ (s, 1H, H-11'), 7.04 (s, 1H, H-12), 7.03 (s, 1H, H-11), 7.01 (s, 1H, H-12'), 6.91 (s, 1H, H-11' '), 6.89 (s, 1H, H-12''), 5.00 (d, J1,2 = 3.6 Hz, 1H, H1), 4.79 (d, $\left.J_{15}{ }^{\prime}, 15^{\prime} \mathrm{a}=13.3 \mathrm{~Hz}, 1 \mathrm{H}, \mathrm{H}-15^{\prime} \mathrm{b}\right), 4.75$ (d, $J_{15}$ ', $15^{\prime}$ 'a $=13.5 \mathrm{~Hz}, 1 \mathrm{H}, \mathrm{H}-15^{\prime}$ 'b), 4.71 $\left(\mathrm{d}, J_{15 \mathrm{~b}, 15 \mathrm{a}}=13.3 \mathrm{~Hz}, 1 \mathrm{H}, \mathrm{H}-15 \mathrm{~b}\right), 4.40$ (m, 1H, H-7a), 4.29 (m, 1H, H-8' 'a), 4.19-4.23 (m, 2H, H-8'a, H-8'b), 4.11-4.18 (m, 2H, H-7b, H-8'’b), 3.96 (d, J' $3^{\prime}, 4^{\prime}=8.3 \mathrm{~Hz}, 1 \mathrm{H}, \mathrm{H}-3^{\prime}$ '), 3.92 (m, 1H, H-7'a), 3.82 (s, 3H, $3 \times \mathrm{H}-16$ '), 3.77 (s, 3H, $3 \times \mathrm{H}-16$ ), 3.77 (s, 3H, $3 \times \mathrm{H}-16$ ''), 3.70-3.78 (m, 3H, H-7'’b, H-1'a, H-6a), 3.62-3.68 (m, 2H, H-4', H-8a), 3.46-3.60 (m, 6H, H3, H-1'b, H-8b, H-15a, H-15'a, H-15'a), 3.30-3.38 (m, 2H, H-4, H-5'), 3.20-3.27 (m, 3H, H2, H-5, H-7'a), 3.01 (m, 1H, H-7'b), 2.74-2.80 (m, 2H, H-6'a, H-6b), 1.73 (dd, J6'b,6'a = 9.2 $\left.\mathrm{Hz}, J_{6}{ }^{\prime}, 5^{\prime}=5.9 \mathrm{~Hz}, 1 \mathrm{H}, \mathrm{H}-6^{\prime}\right) \mathrm{ppm} .{ }^{13} \mathrm{C}\{\mathrm{H}\} \mathrm{NMR}\left(125 \mathrm{MHz}, \mathrm{CD}_{3} \mathrm{OD}\right): \delta=150.56\left(\mathrm{C}-10^{\prime}\right)$, 149.84 (C-10), 148.70 (C-10”), 147.10 (C-9'’), 145.99 (C-9), 144.97 (C-9'), 136.03 (C-13'), 134.87 (C-13), 134.10 (C-14), 133.48 (C-13'’), 132.69 (C-14'’), 132.63 (C-14'), 121.85 (C11 '), 117.96 (C-12), 115.12 (C-11), 114.44 (C-12'), 114.04 (C-12'), 113.51 (C-11”), 105.89 (C-2'), 94.33 (C-1), 80.69 (C-5'), 79.68 (C-4'), 78.15 (C-3'), 75.02 (C-6'), 74.51 (C-3), 73.75 (C-5), 73.45 (C-2), 71.38 (C-6), 71.23 (C-8'), 70.96 (C-8), 70.71 (C-4), 70.40 (C-8'), 70.22 (C-7'”), 69.86 (C-1'), 69.45 (C-7), 67.96 (C-7'), 57.00 (C-16), 56.33 (C-16'), 56.06 (C-16'”), 36.60 (C-15'), 36.50 (C-15), 36.14 (C-15') ppm. HRMS (ESI-TOF) calcd for $\mathrm{C}_{42} \mathrm{H}_{52} \mathrm{O}_{17} \mathrm{~K}$ $[\mathrm{M}+\mathrm{K}]^{+}:$867.2842, found: 867.2832. 


\subsection{Compound $P-9$}
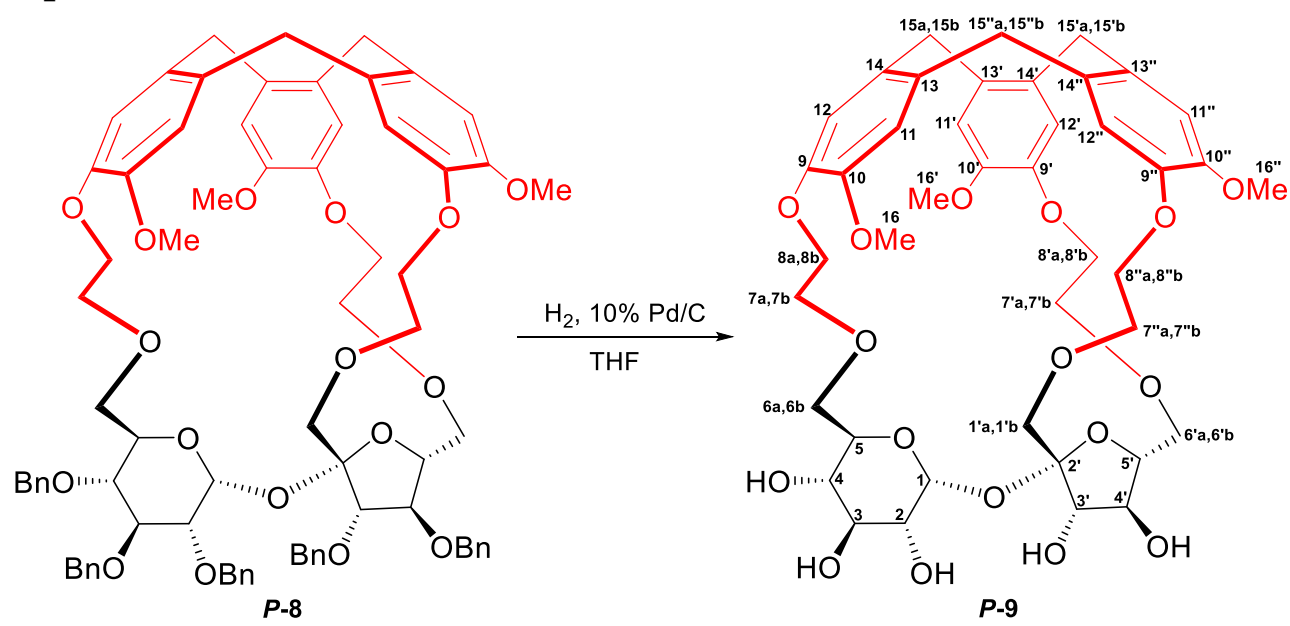

Compound $\boldsymbol{P}$-9 was synthesized according to previously described procedure for compound M-9. Compound $\boldsymbol{P - 8}(48 \mathrm{mg}, 0.038 \mathrm{mmol}), 10 \% \mathrm{Pd} / \mathrm{C}(10 \mathrm{mg}, 0.009 \mathrm{mmol})$ in THF (4 mL) gave unprotected $\boldsymbol{P - 9}(31 \mathrm{mg}, 99 \%)$ as a white solid. $[\alpha]_{\mathrm{D}}=+120.5\left(\mathrm{CHCl}_{3}\right) .{ }^{1} \mathrm{H}$ NMR (500 $\mathrm{MHz}, \mathrm{CD}_{3} \mathrm{OD}$ ): $\delta=7.05$ (s, 1H, H-11'), 7.02 (s, 1H, H-12), 7.00 (s, 1H, H-12')', 6.98 (s, 2H, H-11, H-12'), 6.95 (s, 1H, H-11' '), 4.80 (d, J15b,15a = 13.2 Hz, 1H, H-15b), 4.78 (d, J15'b,15'a = $13.4 \mathrm{~Hz}, 1 \mathrm{H}, \mathrm{H}-15$ 'b), 4.77 (d, $\left.J_{1,2}=3.6 \mathrm{~Hz}, 1 \mathrm{H}, \mathrm{H}-1\right), 4.72$ (d, $J_{15}$ ”b,15"a $=13.4 \mathrm{~Hz}, 1 \mathrm{H}, \mathrm{H}-$ 15' 'b), 4.40 (m, 1H, H-8' 'a), 4.31 (m, 1H, H-8a), 4.22-4.28 (m, 2H, H-8b, H-8'a), 4.16-4.21 (m, 2H, H-8'b, H-8' 'b), 4.03 (d, J3', ' $^{\prime}=8.8 \mathrm{~Hz}, 1 \mathrm{H}, \mathrm{H}-3$ '), 3.85 (s, 3H, $3 \times \mathrm{H}-16^{\prime}$ ') 3.832 (s, $3 \mathrm{H}, 3 \times \mathrm{H}-16$ ), 3.828 (s, 3H, $3 \times \mathrm{H}-16$ '), 3.80 (m, 1H, H-7'’a), 3.73 (m, 1H, H-7' 'b), 3.68 (dd, $J_{4}{ }^{\prime}, 3^{\prime}=8.8 \mathrm{~Hz}, J^{\prime}, 5^{\prime}=8.2 \mathrm{~Hz}, 1 \mathrm{H}, \mathrm{H}-4^{\prime}$ ), 3.51-3.61 (m, 7H, H-15a, H-15'a, H-15'’a, H-7'a, H7a, H-7b, H-1'a), 3.49 (dd, $\left.J_{6 \mathrm{a}, 6 \mathrm{~b}}=12.2 \mathrm{~Hz}, J_{6 \mathrm{a}, 5}=1.5 \mathrm{~Hz}, 1 \mathrm{H}, \mathrm{H}-6 \mathrm{a}\right), 3.45$ (dd, $J_{3,2}=9.7 \mathrm{~Hz}$, $\left.J_{3,4}=8.7 \mathrm{~Hz}, 1 \mathrm{H}, \mathrm{H}-3\right), 3.37-3.41$ (m, 2H, H-4, H-7’b), 3.35 (m, 1H, H-5), 3.27 (dd, $J_{2,3}=9.7$ $\left.\mathrm{Hz}, J_{2,1}=3.6 \mathrm{~Hz}, 1 \mathrm{H}, \mathrm{H}-2\right), 3.23$ (d, $J_{1}{ }^{\prime} \mathrm{b}, 1^{\prime} \mathrm{a}=12.0 \mathrm{~Hz}, 1 \mathrm{H}, \mathrm{H}-1$ 'b), 3.11 (ddd, $J_{5}{ }^{\prime}, 4^{\prime}=8.2 \mathrm{~Hz}$, $\left.J_{5}, 6^{\prime} \mathrm{a}=6.9 \mathrm{~Hz}, J_{5}, 6^{\prime} \mathrm{b}=4.5 \mathrm{~Hz}, 1 \mathrm{H}, \mathrm{H}-5^{\prime}\right), 2.50\left(\mathrm{dd}, J_{6}{ }^{\prime}{ }^{\prime},{ }^{\prime} \mathrm{b}=11.4 \mathrm{~Hz}, J_{6}{ }^{\prime} \mathrm{a}, 5^{\prime}=6.9 \mathrm{~Hz}, 1 \mathrm{H}, \mathrm{H}-\right.$ 6'a), $2.44\left(\mathrm{dd}, J_{6 \mathrm{~b}, 6 \mathrm{a}}=12.2 \mathrm{~Hz}, J_{6 \mathrm{~b}, 5}=1.8 \mathrm{~Hz}, 1 \mathrm{H}, \mathrm{H}-6 \mathrm{~b}\right), 2.15\left(\mathrm{dd}, J_{6}{ }^{\prime} \mathrm{b}, 6^{\prime} \mathrm{a}=11.4 \mathrm{~Hz}, J_{6}{ }^{\prime} \mathrm{b}, 5^{\prime}=\right.$ $4.4 \mathrm{~Hz}, 1 \mathrm{H}, \mathrm{H}-6$ 'b) ppm. ${ }^{13} \mathrm{C}\{\mathrm{H}\} \mathrm{NMR}\left(125 \mathrm{MHz}, \mathrm{CD}_{3} \mathrm{OD}\right): \delta=150.69$ (C-10), $150.40(\mathrm{C}-$ 10'), 148.66 (C-10”), 146.30 (C-9'’), 145.93 (C-9'), 145.55 (C-9), 135.51 (C-13'), 134.98 (C13), 134.46 (C-14), 134.11 (C-13'), 133.20 (C-14'), 132.96 (C-14”), 121.83 (C-11'), 120.69 (C-12'), 115.94 (C-11'’), 115.22 (C-12), 114.42 (C-11), 114.22 (C-12”), 104.60 (C-2'), 94.63 (C-1), 79.65 (C-5'), 78.11 (C-3'), 77.54 (C-4'), $74.69(\mathrm{C}-3), 73.63$ (C-5), 73.35 (C-2), 73.17(C-6'), 71.28 (C-7), 71.17 (C-7'), 70.59 (C-4), 70.44 (C-7'’), 70.44 (C-6), 70.41 (C-8'), 69.21 (C-8), 68.61 (C-1'), 67.58 (C-8'’), 56.93 (C-16), 56.53 (C-16'), 56.25 (C-16’'), 36.69 (C-15”), 36.53 (C-15), 36.37 (C-15') ppm. HRMS (ESI-TOF) calcd for $\mathrm{C}_{42} \mathrm{H}_{52} \mathrm{O}_{17} \mathrm{~K}[\mathrm{M}+$ $\mathrm{K}]^{+}:$867.2842, found: 867.2838 . 

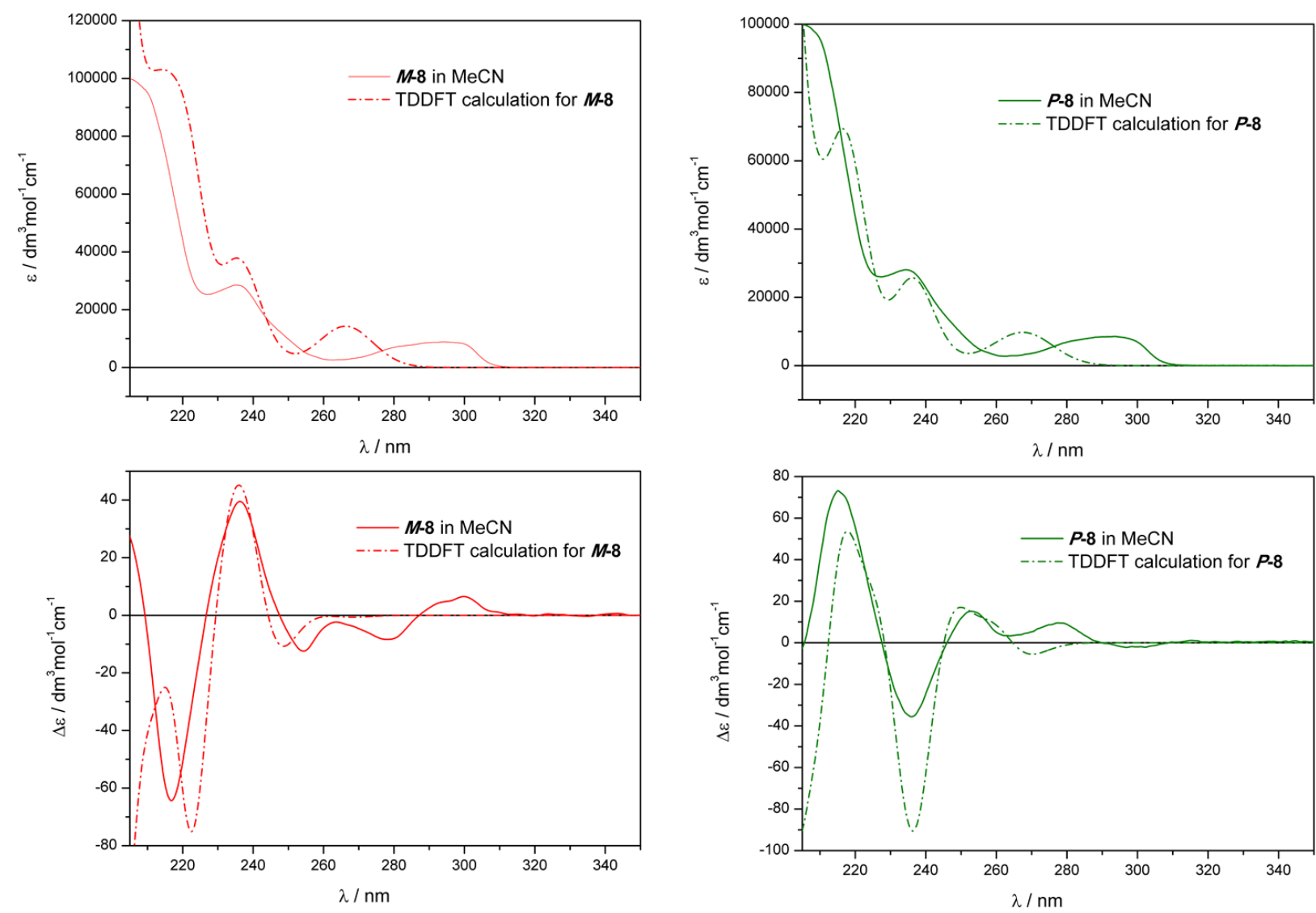

Figure S1.Comparison of experimental UV (top) and ECD (bottom) spectra of compounds $\boldsymbol{M - 8}$ and $\boldsymbol{P}-\mathbf{8}$ in $\mathrm{MeCN}$ solution with the simulated curves calculated at CAMB3LYP/SVP/PCM(MeCN) level of theory. 
Table S1. Calculated at CAM-B3LYP/SVP/PCM $\left(\mathrm{CH}_{3} \mathrm{CN}\right)$ level of theory relative energies and conformer distribution at $25^{\circ} \mathrm{C}$ for $M-8$.

\begin{tabular}{|c|c|c|}
\hline Conformer. & $\begin{array}{c}\Delta G \\
{\left[\mathrm{kcal} \mathrm{mol}^{-1}\right]}\end{array}$ & $\begin{array}{c}\text { Pop. } \\
{[\%]}\end{array}$ \\
\hline $\boldsymbol{M - 8}(1)$ & 0.00 & 43.7 \\
\hline $\boldsymbol{M - 8}(2)$ & 0.46 & 20.2 \\
\hline $\boldsymbol{M - 8}(3)$ & 0.60 & 15.8 \\
\hline $\boldsymbol{M - 8}(4)$ & 0.99 & 8.2 \\
\hline $\boldsymbol{M - 8}(5)$ & 1.13 & 6.4 \\
\hline $\boldsymbol{M}-8(6)$ & 1.21 & 5.7 \\
\hline
\end{tabular}

Cartesian coordinates for individual conformers of compound $M-8$.

Compound $\boldsymbol{M - 8}$, conformer 1

\begin{tabular}{|c|c|c|c|c|c|}
\hline \multirow{2}{*}{$\begin{array}{l}\text { Center } \\
\text { Number }\end{array}$} & \multirow{2}{*}{\multicolumn{2}{|c|}{$\begin{array}{l}\text { Atomic } \\
\text { Number }\end{array}$}} & \multirow{2}{*}{$\begin{array}{r}\text { Atomic } \\
\text { Type }\end{array}$} & \multicolumn{2}{|c|}{ Coordinates (Angstroms } \\
\hline & & & & $\mathrm{X}$ & Z \\
\hline 1 & 6 & 0 & -4.595787 & -1.927003 & 2.696787 \\
\hline 2 & 6 & 0 & -5.340892 & -0.780099 & 2.396877 \\
\hline 3 & 6 & 0 & -4.904630 & 0.452196 & 2.911562 \\
\hline 4 & 6 & 0 & -3.731207 & 0.487324 & 3.676380 \\
\hline 5 & 6 & 0 & -2.970757 & -0.651411 & 3.930932 \\
\hline 6 & 6 & 0 & -3.427503 & -1.887920 & 3.445321 \\
\hline 7 & 1 & 0 & -4.923842 & -2.905747 & 2.340691 \\
\hline 8 & 1 & 0 & -3.406022 & 1.437411 & 4.103947 \\
\hline 9 & 6 & 0 & -5.658107 & 1.756041 & 2.702008 \\
\hline 10 & 1 & 0 & -5.495160 & 2.383434 & 3.589458 \\
\hline 11 & 1 & 0 & -6.736388 & 1.564441 & 2.666166 \\
\hline 12 & 6 & 0 & -6.591441 & -0.937961 & 1.551553 \\
\hline 13 & 1 & 0 & -7.364920 & -0.238196 & 1.887228 \\
\hline 14 & 1 & 0 & -6.997691 & -1.941890 & 1.739563 \\
\hline 15 & 6 & 0 & -5.222826 & 2.552703 & 1.483372 \\
\hline 16 & 6 & 0 & -5.901554 & 2.521284 & 0.253559 \\
\hline 17 & 6 & 0 & -4.083575 & 3.359474 & 1.598887 \\
\hline 18 & 6 & 0 & -5.393590 & 3.275126 & -0.810570 \\
\hline 19 & 6 & 0 & -3.574474 & 4.091631 & 0.532995 \\
\hline 20 & 1 & 0 & -3.549300 & 3.425873 & 2.548824 \\
\hline 21 & 6 & 0 & -4.237010 & 4.034922 & -0.701602 \\
\hline 22 & 1 & 0 & -5.904703 & 3.272759 & -1.775505 \\
\hline 23 & 6 & 0 & -6.387888 & -0.803912 & 0.050294 \\
\hline 24 & 6 & 0 & -5.834494 & -1.885867 & -0.643002 \\
\hline 25 & 6 & 0 & -6.723622 & 0.352012 & -0.674026 \\
\hline 26 & 6 & 0 & -5.622683 & -1.862440 & -2.015862 \\
\hline 27 & 1 & 0 & -5.522431 & -2.784413 & -0.106766 \\
\hline 28 & 6 & 0 & -6.592837 & 0.333935 & -2.066988 \\
\hline 29 & 6 & 0 & -6.064167 & -0.752192 & -2.751361 \\
\hline 30 & 1 & 0 & -6.895899 & 1.197165 & -2.662738 \\
\hline
\end{tabular}




\begin{tabular}{|c|c|c|c|c|c|}
\hline 31 & 6 & 0 & -7.123982 & 1.658306 & -0.016217 \\
\hline 32 & 1 & 0 & -7.792480 & 2.205657 & -0.694985 \\
\hline 33 & 1 & 0 & -7.694240 & 1.485615 & 0.902511 \\
\hline 34 & 8 & 0 & -2.733233 & -3.043992 & 3.661797 \\
\hline 35 & 8 & 0 & -1.831497 & -0.637619 & 4.669046 \\
\hline 36 & 8 & 0 & -2.428254 & 4.812337 & 0.698880 \\
\hline 37 & 8 & 0 & -3.771491 & 4.745635 & -1.774989 \\
\hline 38 & 8 & 0 & -5.940989 & -0.687846 & -4.109480 \\
\hline 39 & 8 & 0 & -4.999038 & -2.925827 & -2.608948 \\
\hline 40 & 6 & 0 & -6.719545 & -1.619707 & -4.843453 \\
\hline 41 & 1 & 0 & -6.528945 & -1.430374 & -5.907331 \\
\hline 42 & 1 & 0 & -6.442054 & -2.657485 & -4.602968 \\
\hline 43 & 1 & 0 & -7.793741 & -1.477205 & -4.640030 \\
\hline 44 & 6 & 0 & -2.685816 & -3.501769 & 5.003909 \\
\hline 45 & 1 & 0 & -2.182937 & -2.774868 & 5.657390 \\
\hline 46 & 1 & 0 & -3.701575 & -3.697908 & 5.386444 \\
\hline 47 & 1 & 0 & -2.117945 & -4.440655 & 5.000954 \\
\hline 48 & 6 & 0 & -2.562332 & 6.222865 & 0.612599 \\
\hline 49 & 1 & 0 & -1.560822 & 6.648660 & 0.750346 \\
\hline 50 & 1 & 0 & -2.955912 & 6.527938 & -0.367627 \\
\hline 51 & 1 & 0 & -3.226633 & 6.601049 & 1.407738 \\
\hline 52 & 6 & 0 & -3.773963 & -2.644644 & -3.277799 \\
\hline 53 & 1 & 0 & -3.941833 & -2.013886 & -4.163876 \\
\hline 54 & 1 & 0 & -3.383396 & -3.616221 & -3.609182 \\
\hline 55 & 6 & 0 & -2.759502 & -1.981666 & -2.360345 \\
\hline 56 & 1 & 0 & -3.089083 & -0.968617 & -2.076300 \\
\hline 57 & 1 & 0 & -2.661473 & -2.564966 & -1.428609 \\
\hline 58 & 6 & 0 & -0.891744 & 0.410707 & 4.496744 \\
\hline 59 & 1 & 0 & -1.243308 & 1.114107 & 3.728633 \\
\hline 60 & 1 & 0 & -0.786275 & 0.975675 & 5.436860 \\
\hline 61 & 6 & 0 & 0.454419 & -0.184391 & 4.117059 \\
\hline 62 & 1 & 0 & 1.173055 & 0.640563 & 3.949968 \\
\hline 63 & 1 & 0 & 0.831685 & -0.796270 & 4.948925 \\
\hline 64 & 6 & 0 & -3.021139 & 4.027410 & -2.743641 \\
\hline 65 & 1 & 0 & -3.394905 & 2.994767 & -2.837435 \\
\hline 66 & 1 & 0 & -3.169279 & 4.532024 & -3.709045 \\
\hline 67 & 6 & 0 & -1.543487 & 4.021006 & -2.397319 \\
\hline 68 & 1 & 0 & -1.387991 & 3.571241 & -1.400678 \\
\hline 69 & 1 & 0 & -1.178044 & 5.063633 & -2.341123 \\
\hline 70 & 8 & 0 & -1.540656 & -1.922011 & -3.066295 \\
\hline 71 & 8 & 0 & 0.386171 & -1.042825 & 3.007154 \\
\hline 72 & 8 & 0 & -0.865333 & 3.313126 & -3.405702 \\
\hline 73 & 6 & 0 & 2.438100 & 3.065281 & -1.704466 \\
\hline 74 & 6 & 0 & 1.181876 & 2.480954 & -2.349716 \\
\hline 75 & 6 & 0 & 1.994826 & 0.245623 & -2.211756 \\
\hline 76 & 6 & 0 & 3.296167 & 0.703345 & -1.532449 \\
\hline 77 & 6 & 0 & 3.099524 & 2.028066 & -0.804079 \\
\hline 78 & 1 & 0 & 0.461815 & 2.268631 & -1.542223 \\
\hline 79 & 1 & 0 & 3.150149 & 3.353944 & -2.499335 \\
\hline 80 & 1 & 0 & 2.189879 & -0.604526 & -2.876544 \\
\hline
\end{tabular}




$\begin{array}{cccccc}81 & 1 & 0 & 4.035405 & 0.871294 & -2.335896 \\ 82 & 1 & 0 & 2.428171 & 1.862329 & 0.055197 \\ 83 & 8 & 0 & 1.502146 & 1.274018 & -3.030624 \\ 84 & 8 & 0 & 1.077587 & -0.096634 & -1.215909 \\ 85 & 8 & 0 & 3.783775 & -0.233290 & -0.610144 \\ 86 & 8 & 0 & 4.339188 & 2.534617 & -0.362491 \\ 87 & 8 & 0 & 2.053184 & 4.191087 & -0.951552 \\ 88 & 6 & 0 & 4.621645 & 2.363460 & 1.008061 \\ 89 & 1 & 0 & 3.891824 & 2.920943 & 1.623578 \\ 90 & 1 & 0 & 4.531497 & 1.296733 & 1.277282 \\ 91 & 6 & 0 & 2.947936 & 5.278871 & -0.961707 \\ 92 & 1 & 0 & 2.967229 & 5.747570 & -1.964776 \\ 93 & 1 & 0 & 3.971056 & 4.928698 & -0.748328 \\ 94 & 6 & 0 & 6.016263 & 2.843502 & 1.314180 \\ 95 & 6 & 0 & 6.336244 & 3.285161 & 2.601254 \\ 96 & 6 & 0 & 7.018001 & 2.818137 & 0.340971 \\ 97 & 6 & 0 & 7.633537 & 3.683622 & 2.913590 \\ 98 & 1 & 0 & 5.558688 & 3.320417 & 3.369118 \\ 99 & 6 & 0 & 8.315062 & 3.223025 & 0.650437 \\ 100 & 1 & 0 & 6.768709 & 2.485053 & -0.667148 \\ 101 & 6 & 0 & 8.628374 & 3.654336 & 1.937640 \\ 102 & 1 & 0 & 7.867149 & 4.026684 & 3.924011 \\ 103 & 1 & 0 & 9.087684 & 3.200997 & -0.121671 \\ 104 & 1 & 0 & 9.645091 & 3.971647 & 2.179538 \\ 105 & 6 & 0 & 2.545171 & 6.305785 & 0.065543 \\ 106 & 6 & 0 & 1.489658 & 6.088314 & 0.951592 \\ 107 & 6 & 0 & 3.262555 & 7.504386 & 0.148270 \\ 108 & 6 & 0 & 1.161968 & 7.050849 & 1.908036 \\ 109 & 1 & 0 & 0.921309 & 5.160552 & 0.884508 \\ 110 & 6 & 0 & 2.934798 & 8.464196 & 1.100087 \\ 111 & 1 & 0 & 4.090590 & 7.686464 & -0.542552 \\ 112 & 6 & 0 & 1.881067 & 8.239832 & 1.986651 \\ 113 & 1 & 0 & 0.336086 & 6.865657 & 2.598938 \\ 114 & 1 & 0 & 3.505195 & 9.394370 & 1.151454 \\ 115 & 1 & 0 & 1.623083 & 8.991579 & 2.735707 \\ 116 & 6 & 0 & 0.535468 & 3.429266 & -3.349047 \\ 117 & 1 & 0 & 0.816112 & 4.466900 & -3.101060 \\ 118 & 1 & 0 & 0.926832 & 3.189888 & -4.348714 \\ 119 & 6 & 0 & 0.066920 & -1.074664 & -1.430064 \\ 120 & 8 & 0 & -0.837188 & -0.915502 & -0.384568 \\ 121 & 6 & 0 & -0.329443 & -1.536381 & 0.802050 \\ 122 & 6 & 0 & 0.760289 & -2.525807 & 0.331421 \\ 123 & 6 & 0 & 0.614694 & -2.481813 & -1.188479 \\ 124 & 1 & 0 & -1.151226 & -2.102157 & 1.267048 \\ 125 & 1 & 0 & 1.765467 & -2.166836 & 0.611480 \\ 126 & 1 & 0 & -0.180343 & -3.198105 & -1.460792 \\ 127 & 6 & 0 & -0.666274 & -0.873287 & -2.755976 \\ 128 & 1 & 0 & -1.188319 & 0.099645 & -2.713310 \\ 129 & 1 & 0 & 0.064195 & -0.824125 & -3.572838 \\ 130 & 8 & 0 & 1.763901 & -2.733123 & -1.938438\end{array}$




$\begin{array}{rrrrrr}131 & 8 & 0 & 0.564892 & -3.847196 & 0.764567 \\ 132 & 6 & 0 & 0.953712 & -4.100915 & 2.105296 \\ 133 & 1 & 0 & 0.443765 & -3.395124 & 2.780668 \\ 134 & 1 & 0 & 2.041184 & -3.938771 & 2.216185 \\ 135 & 6 & 0 & 0.591848 & -5.519325 & 2.447410 \\ 136 & 6 & 0 & 1.562366 & -6.521062 & 2.500940 \\ 137 & 6 & 0 & -0.745595 & -5.858420 & 2.681319 \\ 138 & 6 & 0 & 1.208624 & -7.839247 & 2.787698 \\ 139 & 1 & 0 & 2.609338 & -6.265423 & 2.318022 \\ 140 & 6 & 0 & -1.102414 & -7.174155 & 2.962888 \\ 141 & 1 & 0 & -1.509063 & -5.076267 & 2.650585 \\ 142 & 6 & 0 & -0.124852 & -8.168224 & 3.017098 \\ 143 & 1 & 0 & 1.978894 & -8.612564 & 2.829912 \\ 144 & 1 & 0 & -2.149251 & -7.427121 & 3.146073 \\ 145 & 1 & 0 & -0.404399 & -9.200111 & 3.241158 \\ 146 & 6 & 0 & 4.643339 & -1.214633 & -1.134316 \\ 147 & 1 & 0 & 4.137019 & -1.792420 & -1.926817 \\ 148 & 1 & 0 & 5.528645 & -0.734401 & -1.593608 \\ 149 & 6 & 0 & 5.091482 & -2.160848 & -0.049521 \\ 150 & 6 & 0 & 4.656335 & -2.035110 & 1.270194 \\ 151 & 6 & 0 & 5.970364 & -3.200606 & -0.374468 \\ 152 & 6 & 0 & 5.089527 & -2.933411 & 2.247030 \\ 153 & 1 & 0 & 3.971646 & -1.228080 & 1.531012 \\ 154 & 6 & 0 & 6.402136 & -4.096366 & 0.598258 \\ 155 & 1 & 0 & 6.318080 & -3.310948 & -1.405047 \\ 156 & 6 & 0 & 5.961597 & -3.966317 & 1.916050 \\ 157 & 1 & 0 & 4.740480 & -2.820981 & 3.276125 \\ 158 & 1 & 0 & 7.088173 & -4.902094 & 0.327378 \\ 159 & 1 & 0 & 6.300995 & -4.667969 & 2.681062 \\ 160 & 6 & 0 & 3.100393 & -4.309300 & -3.143479 \\ 161 & 6 & 0 & 2.851845 & -3.695551 & -4.374915 \\ 162 & 6 & 0 & 4.194878 & -5.168678 & -3.025913 \\ 163 & 6 & 0 & 3.681906 & -3.937656 & -5.466158 \\ 164 & 1 & 0 & 1.999238 & -3.020939 & -4.471852 \\ 165 & 6 & 0 & 5.022241 & -5.419006 & -4.119579 \\ 166 & 1 & 0 & 4.405748 & -5.644655 & -2.065075 \\ 167 & 6 & 0 & 4.768994 & -4.802211 & -5.342491 \\ 168 & 1 & 0 & 3.477501 & -3.449926 & -6.422000 \\ 169 & 1 & 0 & 5.874125 & -6.094146 & -4.012184 \\ 170 & 1 & 0 & 5.419497 & -4.992692 & -6.198902 \\ 171 & 6 & 0 & 2.178482 & -4.083960 & -1.973649 \\ 172 & 1 & 0 & 1.288348 & -4.734663 & -2.066236 \\ 173 & 1 & 0 & 2.678941 & -4.364959 & -1.031912 \\ 174 & 6 & 0 & 0.114633 & -0.447517 & 1.765355 \\ 175 & 1 & 0 & -0.706720 & 0.285767 & 1.828850 \\ 176 & 1 & 0 & 1.002868 & 0.076290 & 1.369605 \\ ---------------------------------------------------------\end{array}$

Compound $\boldsymbol{M - 8}$, conformer 2 


\begin{tabular}{|c|c|c|c|c|c|}
\hline \multirow{2}{*}{$\begin{array}{l}\text { Center } \\
\text { Number }\end{array}$} & Atomic & \multirow{2}{*}{\multicolumn{2}{|c|}{$\begin{array}{r}\text { Atomic } \\
\text { Type }\end{array}$}} & \multicolumn{2}{|c|}{ Coordinates (Angstrom } \\
\hline & Numbe & & & $\mathrm{X}$ & $\mathrm{Z}$ \\
\hline 1 & 6 & 0 & -4.652068 & -1.541195 & 3.282535 \\
\hline 2 & 6 & 0 & -5.566789 & -0.686133 & 2.664773 \\
\hline 3 & 6 & 0 & -5.353962 & 0.701301 & 2.754023 \\
\hline 4 & 6 & 0 & -4.246448 & 1.155975 & 3.477561 \\
\hline 5 & 6 & 0 & -3.325255 & 0.296730 & 4.074612 \\
\hline 6 & 6 & 0 & -3.526868 & -1.088878 & 3.968019 \\
\hline 7 & 1 & 0 & -4.797646 & -2.622563 & 3.246479 \\
\hline 8 & 1 & 0 & -4.087870 & 2.230251 & 3.595147 \\
\hline 9 & 6 & 0 & -6.266730 & 1.730191 & 2.107133 \\
\hline 10 & 1 & 0 & -6.234800 & 2.643896 & 2.716810 \\
\hline 11 & 1 & 0 & -7.307130 & 1.386696 & 2.136394 \\
\hline 12 & 6 & 0 & -6.760753 & -1.309600 & 1.963385 \\
\hline 13 & 1 & 0 & -7.629344 & -0.646291 & 2.039238 \\
\hline 14 & 1 & 0 & -7.032285 & -2.219048 & 2.517689 \\
\hline 15 & 6 & 0 & -5.871411 & 2.100680 & 0.686308 \\
\hline 16 & 6 & 0 & -6.453165 & 1.526213 & -0.456687 \\
\hline 17 & 6 & 0 & -4.865321 & 3.060603 & 0.514129 \\
\hline 18 & 6 & 0 & -5.978271 & 1.911611 & -1.715275 \\
\hline 19 & 6 & 0 & -4.389828 & 3.429574 & -0.738663 \\
\hline 20 & 1 & 0 & -4.413170 & 3.545636 & 1.381744 \\
\hline 21 & 6 & 0 & -4.946789 & 2.825924 & -1.875384 \\
\hline 22 & 1 & 0 & -6.416111 & 1.483151 & -2.619208 \\
\hline 23 & 6 & 0 & -6.536351 & -1.708791 & 0.514786 \\
\hline 24 & 6 & 0 & -5.868729 & -2.909814 & 0.253333 \\
\hline 25 & 6 & 0 & -6.961108 & -0.928373 & -0.573142 \\
\hline 26 & 6 & 0 & -5.617534 & -3.357858 & -1.037356 \\
\hline 27 & 1 & 0 & -5.499042 & -3.526989 & 1.074510 \\
\hline 28 & 6 & 0 & -6.776176 & -1.424206 & -1.868324 \\
\hline 29 & 6 & 0 & -6.123623 & -2.624220 & -2.120729 \\
\hline 30 & 1 & 0 & -7.139548 & -0.864628 & -2.732562 \\
\hline 31 & 6 & 0 & -7.540065 & 0.464170 & -0.415514 \\
\hline 32 & 1 & 0 & -8.235159 & 0.650881 & -1.245800 \\
\hline 33 & 1 & 0 & -8.132163 & 0.545253 & 0.502064 \\
\hline 34 & 8 & 0 & -2.686973 & -2.039326 & 4.449503 \\
\hline 35 & 8 & 0 & -2.267978 & 0.771907 & 4.793321 \\
\hline 36 & 8 & 0 & -3.374519 & 4.336197 & -0.834659 \\
\hline 37 & 8 & 0 & -4.505674 & 3.162525 & -3.126865 \\
\hline 38 & 8 & 0 & -5.954446 & -3.031698 & -3.412679 \\
\hline 39 & 8 & 0 & -4.896629 & -4.507760 & -1.212587 \\
\hline 40 & 6 & 0 & -6.623426 & -4.229547 & -3.774692 \\
\hline 41 & 1 & 0 & -6.404532 & -4.412205 & -4.834297 \\
\hline 42 & 1 & 0 & -6.272873 & -5.085108 & -3.177503 \\
\hline 43 & 1 & 0 & -7.713268 & -4.122220 & -3.646535 \\
\hline 44 & 6 & 0 & -2.051204 & -1.880680 & 5.705970 \\
\hline 45 & 1 & 0 & -1.133506 & -1.286710 & 5.619180 \\
\hline 46 & 1 & 0 & -2.723797 & -1.403415 & 6.435256 \\
\hline 47 & 1 & 0 & -1.803971 & -2.891738 & 6.057637 \\
\hline
\end{tabular}




$\begin{array}{rrrrrr}48 & 6 & 0 & -3.702844 & 5.568780 & -1.459473 \\ 49 & 1 & 0 & -4.049068 & 5.413324 & -2.490937 \\ 50 & 1 & 0 & -4.481510 & 6.099872 & -0.886790 \\ 51 & 1 & 0 & -2.789180 & 6.175100 & -1.468353 \\ 52 & 6 & 0 & -3.658233 & -4.371099 & -1.901384 \\ 53 & 1 & 0 & -3.817982 & -4.036622 & -2.937839 \\ 54 & 1 & 0 & -3.212971 & -5.374946 & -1.923157 \\ 55 & 6 & 0 & -2.715143 & -3.413651 & -1.191432 \\ 56 & 1 & 0 & -3.115340 & -2.387145 & -1.210012 \\ 57 & 1 & 0 & -2.613379 & -3.703306 & -0.131368 \\ 58 & 6 & 0 & -1.357746 & 1.668720 & 4.178387 \\ 59 & 1 & 0 & -1.715651 & 1.945154 & 3.175530 \\ 60 & 1 & 0 & -1.300006 & 2.594907 & 4.772440 \\ 61 & 6 & 0 & 0.025754 & 1.040924 & 4.122884 \\ 62 & 1 & 0 & 0.715999 & 1.746671 & 3.622119 \\ 63 & 1 & 0 & 0.393425 & 0.877155 & 5.145908 \\ 64 & 6 & 0 & -3.630148 & 2.244553 & -3.764083 \\ 65 & 1 & 0 & -3.927154 & 1.205710 & -3.544939 \\ 66 & 1 & 0 & -3.727806 & 2.405600 & -4.846882 \\ 67 & 6 & 0 & -2.190339 & 2.476474 & -3.344825 \\ 68 & 1 & 0 & -1.900385 & 3.514453 & -3.593557 \\ 69 & 1 & 0 & -2.089493 & 2.366283 & -2.250114 \\ 70 & 8 & 0 & -1.475364 & -3.472205 & -1.861225 \\ 71 & 8 & 0 & 0.044534 & -0.220152 & 3.498606 \\ 72 & 8 & 0 & -1.384669 & 1.556948 & -4.038827 \\ 73 & 6 & 0 & 1.742110 & 2.209664 & -2.156140 \\ 74 & 6 & 0 & 0.637263 & 1.287317 & -2.674140 \\ 75 & 6 & 0 & 1.731980 & -0.666002 & -1.852447 \\ 76 & 6 & 0 & 2.938529 & 0.150478 & -1.357928 \\ 77 & 6 & 0 & 2.490445 & 1.561636 & -0.996829 \\ 78 & 1 & 0 & -0.129558 & 1.221489 & -1.884665 \\ 79 & 1 & 0 & 2.459621 & 2.411863 & -2.972695 \\ 80 & 1 & 0 & 2.053118 & -1.647779 & -2.221095 \\ 81 & 1 & 0 & 3.645394 & 0.217619 & -2.203488 \\ 82 & 1 & 0 & 1.789697 & 1.498435 & -0.148367 \\ 83 & 8 & 0 & 1.148610 & -0.011205 & -2.949219 \\ 84 & 8 & 0 & 0.825467 & -0.794847 & -0.796604 \\ 85 & 8 & 0 & 3.568855 & -0.408762 & -0.235597 \\ 86 & 8 & 0 & 3.590068 & 2.373446 & -0.651840 \\ 87 & 8 & 0 & 1.123249 & 3.401201 & -1.730698 \\ 88 & 6 & 0 & 3.757365 & 2.609081 & 0.725029 \\ 89 & 1 & 0 & 2.859879 & 3.108949 & 1.136632 \\ 90 & 1 & 0 & 3.869093 & 1.650524 & 1.261654 \\ 91 & 6 & 0 & 1.835426 & 4.596029 & -1.959069 \\ 92 & 1 & 0 & 1.787727 & 4.863283 & -3.033552 \\ 93 & 1 & 0 & 2.897779 & 4.464132 & -1.703052 \\ 94 & 6 & 0 & 4.967167 & 3.473351 & 0.973763 \\ 95 & 6 & 0 & 5.234018 & 3.915408 & 2.274544 \\ 96 & 6 & 0 & 5.838737 & 3.836546 & -0.053700 \\ 97 & 6 & 0 & 6.349900 & 4.701425 & 2.542425\end{array}$




$\begin{array}{cccccc}98 & 1 & 0 & 4.556155 & 3.641203 & 3.087821 \\ 99 & 6 & 0 & 6.957414 & 4.627050 & 0.214279 \\ 100 & 1 & 0 & 5.634808 & 3.492120 & -1.067466 \\ 101 & 6 & 0 & 7.217707 & 5.061130 & 1.510609 \\ 102 & 1 & 0 & 6.543628 & 5.038520 & 3.563227 \\ 103 & 1 & 0 & 7.629817 & 4.904674 & -0.600843 \\ 104 & 1 & 0 & 8.093285 & 5.679608 & 1.719495 \\ 105 & 6 & 0 & 1.261479 & 5.718356 & -1.131935 \\ 106 & 6 & 0 & 0.024829 & 5.605962 & -0.494578 \\ 107 & 6 & 0 & 1.983987 & 6.909368 & -1.001569 \\ 108 & 6 & 0 & -0.475834 & 6.664913 & 0.263773 \\ 109 & 1 & 0 & -0.550247 & 4.684976 & -0.592082 \\ 110 & 6 & 0 & 1.480719 & 7.968132 & -0.252036 \\ 111 & 1 & 0 & 2.957585 & 7.005957 & -1.490158 \\ 112 & 6 & 0 & 0.246836 & 7.848336 & 0.387354 \\ 113 & 1 & 0 & -1.442133 & 6.558217 & 0.762207 \\ 114 & 1 & 0 & 2.058612 & 8.890480 & -0.159555 \\ 115 & 1 & 0 & -0.147099 & 8.675394 & 0.982045 \\ 116 & 6 & 0 & -0.005954 & 1.817068 & -3.949657 \\ 117 & 1 & 0 & 0.174852 & 2.902781 & -4.028913 \\ 118 & 1 & 0 & 0.479111 & 1.320561 & -4.802850 \\ 119 & 6 & 0 & -0.025031 & -1.927302 & -0.646595 \\ 120 & 8 & 0 & -0.967859 & -1.563269 & 0.309330 \\ 121 & 6 & 0 & -0.383276 & -1.638168 & 1.619559 \\ 122 & 6 & 0 & 0.900291 & -2.486572 & 1.453055 \\ 123 & 6 & 0 & 0.721154 & -3.063981 & 0.049733 \\ 124 & 1 & 0 & -1.085237 & -2.165109 & 2.284183 \\ 125 & 1 & 0 & 1.792690 & -1.840702 & 1.448980 \\ 126 & 1 & 0 & 0.033317 & -3.925575 & 0.118031 \\ 127 & 6 & 0 & -0.736134 & -2.287139 & -1.947709 \\ 128 & 1 & 0 & -1.366946 & -1.428706 & -2.240803 \\ 129 & 1 & 0 & 0.011092 & -2.438458 & -2.737161 \\ 130 & 8 & 0 & 1.880470 & -3.425808 & -0.636211 \\ 131 & 8 & 0 & 1.034835 & -3.530636 & 2.382491 \\ 132 & 6 & 0 & 1.552327 & -3.155988 & 3.652542 \\ 133 & 1 & 0 & 1.426292 & -4.044691 & 4.286888 \\ 134 & 1 & 0 & 0.955425 & -2.334353 & 4.078412 \\ 135 & 6 & 0 & 3.010024 & -2.758936 & 3.599867 \\ 136 & 6 & 0 & 3.392747 & -1.419965 & 3.711328 \\ 137 & 6 & 0 & 3.999380 & -3.727537 & 3.396292 \\ 138 & 6 & 0 & 4.735465 & -1.052953 & 3.619683 \\ 139 & 1 & 0 & 2.622452 & -0.660258 & 3.863016 \\ 140 & 6 & 0 & 5.340380 & -3.365807 & 3.304135 \\ 141 & 1 & 0 & 3.713196 & -4.779651 & 3.316026 \\ 142 & 6 & 0 & 5.711287 & -2.024941 & 3.413810 \\ 143 & 1 & 0 & 5.019230 & -0.001620 & 3.706678 \\ 144 & 1 & 0 & 6.102561 & -4.133027 & 3.150334 \\ 146 & 1 & 0 & 6.763182 & -1.740001 & 3.341535 \\ 147 & 1 & 0 & 4.469147 & -1.471459 & -0.487459 \\ & & & & & \\ 145 & & 0.707638 & -1.881305 & 0.506653\end{array}$




$\begin{array}{rrrrrr}148 & 1 & 0 & 3.976230 & -2.276808 & -1.050439 \\ 149 & 6 & 0 & 5.746949 & -1.059637 & -1.183823 \\ 150 & 6 & 0 & 6.347847 & -1.920791 & -2.105805 \\ 151 & 6 & 0 & 6.371669 & 0.155279 & -0.888862 \\ 152 & 6 & 0 & 7.557102 & -1.581851 & -2.711175 \\ 153 & 1 & 0 & 5.864640 & -2.870187 & -2.352269 \\ 154 & 6 & 0 & 7.574369 & 0.500175 & -1.499824 \\ 155 & 1 & 0 & 5.899796 & 0.842233 & -0.185700 \\ 156 & 6 & 0 & 8.173703 & -0.369422 & -2.410493 \\ 157 & 1 & 0 & 8.015132 & -2.266911 & -3.428299 \\ 158 & 1 & 0 & 8.046906 & 1.456012 & -1.261457 \\ 159 & 1 & 0 & 9.118072 & -0.099581 & -2.888446 \\ 160 & 6 & 0 & 3.511157 & -5.060161 & -1.246263 \\ 161 & 6 & 0 & 3.280961 & -4.937160 & -2.620280 \\ 162 & 6 & 0 & 4.733467 & -5.574427 & -0.809653 \\ 163 & 6 & 0 & 4.254801 & -5.321415 & -3.537581 \\ 164 & 1 & 0 & 2.329123 & -4.530944 & -2.968329 \\ 165 & 6 & 0 & 5.707156 & -5.968270 & -1.726639 \\ 166 & 1 & 0 & 4.929088 & -5.662092 & 0.262062 \\ 167 & 6 & 0 & 5.471033 & -5.840673 & -3.093119 \\ 168 & 1 & 0 & 4.063393 & -5.218095 & -4.608033 \\ 169 & 1 & 0 & 6.658678 & -6.368392 & -1.369710 \\ 170 & 1 & 0 & 6.234419 & -6.143462 & -3.813100 \\ 171 & 6 & 0 & 2.450124 & -4.663563 & -0.254277 \\ 172 & 1 & 0 & 1.653944 & -5.432134 & -0.221888 \\ 173 & 1 & 0 & 2.876349 & -4.600415 & 0.758861 \\ 174 & 6 & 0 & -0.220311 & -0.212179 & 2.119448 \\ 175 & 1 & 0 & -1.164986 & 0.309120 & 1.891703 \\ 176 & 1 & 0 & 0.586035 & 0.299923 & 1.564679 \\ -----------------------------------------------------------\end{array}$

Compound $\boldsymbol{M - 8}$, conformer 3

\begin{tabular}{|c|c|c|c|c|}
\hline \multirow{2}{*}{$\begin{array}{l}\text { Center } \\
\text { Number }\end{array}$} & Atomic & \multirow{2}{*}{$\begin{array}{l}\text { Atomic } \\
\text { Type }\end{array}$} & \multicolumn{2}{|c|}{ Coordinates (Angstroms) } \\
\hline & Number & & $X$ & $\mathrm{Z}$ \\
\hline 1 & 6 & -3.790290 & 2.492733 & 3.347959 \\
\hline 2 & 1 & -3.941692 & 1.830806 & 4.214046 \\
\hline 3 & 1 & -3.411525 & 3.458645 & 3.708333 \\
\hline 4 & 6 & -2.771663 & 1.876471 & 2.402636 \\
\hline 5 & 1 & -3.082731 & 0.865071 & 2.092822 \\
\hline 6 & 1 & -2.693933 & 2.487837 & 1.487054 \\
\hline 7 & 6 & -0.927831 & -0.250745 & -4.535584 \\
\hline 8 & 1 & -1.266592 & -0.983089 & -3.789065 \\
\hline 9 & 1 & -0.827357 & -0.782029 & -5.495732 \\
\hline 10 & 6 & 0.417667 & 0.340682 & -4.148043 \\
\hline 11 & 1 & 1.143884 & -0.484330 & -4.018514 \\
\hline 12 & 1 & 0.781931 & 0.985052 & -4.960984 \\
\hline 13 & 6 & -2.933370 & -4.090642 & 2.591201 \\
\hline 14 & 1 & -3.303455 & -3.056255 & 2.676874 \\
\hline
\end{tabular}




\begin{tabular}{rrrrrr}
15 & 1 & 0 & -3.110127 & -4.595542 & 3.551918 \\
16 & 6 & 0 & -1.446874 & -4.090502 & 2.285171 \\
17 & 1 & 0 & -1.263346 & -3.652859 & 1.287827 \\
18 & 1 & 0 & -1.081225 & -5.134271 & 2.250198 \\
19 & 8 & 0 & -1.545798 & 1.823146 & 3.096807 \\
20 & 8 & 0 & 0.355033 & 1.158039 & -3.007122 \\
21 & 8 & 0 & -0.797067 & -3.373572 & 3.305786 \\
22 & 6 & 0 & 2.528041 & -3.063038 & 1.655851 \\
23 & 6 & 0 & 1.253908 & -2.506441 & 2.290062 \\
24 & 6 & 0 & 2.024218 & -0.253560 & 2.176158 \\
25 & 6 & 0 & 3.330279 & -0.681938 & 1.485501 \\
26 & 6 & 0 & 3.159859 & -2.008493 & 0.754306 \\
27 & 1 & 0 & 0.539727 & -2.298423 & 1.476162 \\
28 & 1 & 0 & 3.243794 & -3.323795 & 2.457561 \\
29 & 1 & 0 & 2.210336 & 0.589443 & 2.852386 \\
30 & 1 & 0 & 4.077761 & -0.837209 & 2.283917 \\
31 & 1 & 0 & 2.478839 & -1.857026 & -0.100105 \\
32 & 8 & 0 & 1.549261 & -1.299367 & 2.983033 \\
33 & 8 & 0 & 1.096763 & 0.092186 & 1.190379 \\
34 & 8 & 0 & 3.792928 & 0.268719 & 0.564667 \\
35 & 8 & 0 & 4.408143 & -2.483022 & 0.301925 \\
36 & 8 & 0 & 2.184466 & -4.204177 & 0.906396 \\
37 & 6 & 0 & 4.664526 & -2.319582 & -1.074554 \\
38 & 1 & 0 & 3.915444 & -2.869319 & -1.673394 \\
39 & 1 & 0 & 4.581700 & -1.252334 & -1.345605 \\
40 & 6 & 0 & 3.120441 & -5.270842 & 0.949698 \\
41 & 1 & 0 & 3.189878 & -5.657428 & 1.983702 \\
42 & 1 & 0 & 4.116468 & -4.909893 & 0.654159 \\
43 & 6 & 0 & 6.045748 & -2.820521 & -1.405997 \\
44 & 6 & 0 & 6.322433 & -3.319181 & -2.682235 \\
45 & 6 & 0 & 7.078159 & -2.760559 & -0.466452 \\
46 & 6 & 0 & 7.607509 & -3.739427 & -3.017798 \\
47 & 1 & 0 & 5.520801 & -3.381258 & -3.423136 \\
48 & 6 & 0 & 8.362319 & -3.186816 & -0.798740 \\
49 & 1 & 0 & 6.862991 & -2.383497 & 0.534130 \\
50 & 6 & 0 & 8.632520 & -3.674778 & -2.075797 \\
51 & 1 & 0 & 7.807272 & -4.126933 & -4.019349 \\
52 & 1 & 0 & 9.159191 & -3.137020 & -0.053048 \\
53 & 1 & 0 & 9.639432 & -4.008806 & -2.335485 \\
54 & 6 & 0 & 2.659209 & -6.359025 & 0.020021 \\
55 & 6 & 0 & 3.261084 & -6.535776 & -1.227652 \\
56 & 6 & 0 & 1.593119 & -7.190141 & 0.378250 \\
57 & 6 & 0 & 2.811232 & -7.524842 & -2.101171 \\
58 & 1 & 0 & 4.095485 & -5.892122 & -1.518136 \\
59 & 6 & 0 & 1.138499 & -8.177169 & -0.491944 \\
64 & 1 & 0 & 1.117263 & -7.063957 & 1.354146 \\
& 6 & 0 & 1.747783 & -8.346253 & -1.735474 \\
& 1 & 0 & 3.294171 & -7.653652 & -3.072302 \\
63 & 0 & 0.308177 & -8.822821 & -0.197357 \\
\hline 0 & & & 1.394505 & -9.122677 & -2.417615
\end{tabular}




$\begin{array}{rrrrrr}65 & 6 & 0 & 0.606468 & -3.468810 & 3.274697 \\ 66 & 1 & 0 & 0.907477 & -4.501024 & 3.028942 \\ 67 & 1 & 0 & 0.975528 & -3.226728 & 4.282134 \\ 68 & 6 & 0 & 0.070296 & 1.046878 & 1.433545 \\ 69 & 8 & 0 & -0.836567 & 0.899467 & 0.388617 \\ 70 & 6 & 0 & -0.351685 & 1.566248 & -0.782398 \\ 71 & 6 & 0 & 0.724846 & 2.559880 & -0.291475 \\ 72 & 6 & 0 & 0.591328 & 2.470084 & 1.227348 \\ 73 & 1 & 0 & -1.187827 & 2.131689 & -1.221640 \\ 74 & 1 & 0 & 1.733700 & 2.225799 & -0.589087 \\ 75 & 1 & 0 & -0.214428 & 3.163678 & 1.525665 \\ 76 & 6 & 0 & -0.653683 & 0.798716 & 2.756912 \\ 77 & 1 & 0 & -1.157733 & -0.182405 & 2.692161 \\ 78 & 1 & 0 & 0.080884 & 0.742525 & 3.569551 \\ 79 & 8 & 0 & 1.742033 & 2.721990 & 1.975117 \\ 80 & 8 & 0 & 0.504923 & 3.889854 & -0.684763 \\ 81 & 6 & 0 & 0.876799 & 4.189100 & -2.020804 \\ 82 & 1 & 0 & 0.362849 & 3.502669 & -2.712888 \\ 83 & 1 & 0 & 1.963694 & 4.036741 & -2.149632 \\ 84 & 6 & 0 & 0.503359 & 5.615809 & -2.312343 \\ 85 & 6 & 0 & 1.468908 & 6.622847 & -2.354694 \\ 86 & 6 & 0 & -0.839554 & 5.955967 & -2.510708 \\ 87 & 6 & 0 & 1.104833 & 7.947342 & -2.595984 \\ 88 & 1 & 0 & 2.520135 & 6.366460 & -2.199283 \\ 89 & 6 & 0 & -1.206628 & 7.277801 & -2.746725 \\ 90 & 1 & 0 & -1.599117 & 5.169779 & -2.488709 \\ 91 & 6 & 0 & -0.233968 & 8.277204 & -2.790345 \\ 92 & 1 & 0 & 1.871283 & 8.724848 & -2.630051 \\ 93 & 1 & 0 & -2.257708 & 7.531515 & -2.902530 \\ 94 & 1 & 0 & -0.521555 & 9.313993 & -2.978768 \\ 95 & 6 & 0 & 4.635488 & 1.264601 & 1.088822 \\ 96 & 1 & 0 & 4.125245 & 1.822704 & 1.892756 \\ 97 & 1 & 0 & 5.537032 & 0.800685 & 1.533241 \\ 98 & 6 & 0 & 5.048785 & 2.231751 & 0.008719 \\ 99 & 6 & 0 & 4.605600 & 2.105406 & -1.308260 \\ 100 & 6 & 0 & 5.902353 & 3.291938 & 0.335185 \\ 101 & 6 & 0 & 5.006177 & 3.023083 & -2.280972 \\ 102 & 1 & 0 & 3.940082 & 1.282704 & -1.569714 \\ 103 & 6 & 0 & 6.301670 & 4.206945 & -0.633474 \\ 104 & 1 & 0 & 6.255799 & 3.402958 & 1.363733 \\ 105 & 6 & 0 & 5.853355 & 4.076082 & -1.948590 \\ 106 & 1 & 0 & 4.651107 & 2.910099 & -3.307942 \\ 107 & 1 & 0 & 6.968233 & 5.028512 & -0.361538 \\ 108 & 1 & 0 & 6.167274 & 4.792892 & -2.710430 \\ 109 & 6 & 0 & 3.076261 & 4.281710 & 3.202564 \\ 110 & 6 & 0 & 2.870972 & 3.621693 & 4.417984 \\ 111 & 6 & 0 & 4.153592 & 5.162594 & 3.086028 \\ 112 & 6 & 0 & 3.726882 & 3.839122 & 5.494304 \\ 113 & 1 & 0 & 2.031898 & 2.930172 & 4.514086 \\ 114 & 6 & 0 & 5.006806 & 5.388170 & 4.165177\end{array}$




$\begin{array}{rrrrrr}115 & 1 & 0 & 4.330609 & 5.675151 & 2.137237 \\ 116 & 6 & 0 & 4.796885 & 4.724966 & 5.371874 \\ 117 & 1 & 0 & 3.556372 & 3.315155 & 6.437529 \\ 118 & 1 & 0 & 5.844903 & 6.080498 & 4.058756 \\ 119 & 1 & 0 & 5.467742 & 4.896014 & 6.216603 \\ 120 & 6 & 0 & 2.127642 & 4.079839 & 2.050057 \\ 121 & 1 & 0 & 1.226086 & 4.705902 & 2.190878 \\ 122 & 1 & 0 & 2.595656 & 4.407159 & 1.106716 \\ 123 & 6 & 0 & 0.102609 & 0.516932 & -1.783963 \\ 124 & 1 & 0 & -0.706893 & -0.227528 & -1.866265 \\ 125 & 1 & 0 & 1.002352 & -0.004759 & -1.411811 \\ 126 & 6 & 0 & -4.034005 & -3.312406 & -1.724911 \\ 127 & 6 & 0 & -5.197104 & -2.546392 & -1.577272 \\ 128 & 6 & 0 & -5.867453 & -2.574532 & -0.342991 \\ 129 & 6 & 0 & -5.332593 & -3.351296 & 0.691355 \\ 130 & 6 & 0 & -4.153862 & -4.071171 & 0.551364 \\ 131 & 6 & 0 & -3.494318 & -4.060888 & -0.685973 \\ 132 & 1 & 0 & -3.501076 & -3.328027 & -2.677739 \\ 133 & 1 & 0 & -5.837609 & -3.396246 & 1.658476 \\ 134 & 6 & 0 & -7.104351 & -1.745922 & -0.034046 \\ 135 & 1 & 0 & -7.756730 & -2.328358 & 0.631084 \\ 136 & 1 & 0 & -7.685590 & -1.551922 & -0.941553 \\ 137 & 6 & 0 & -5.660086 & -1.720519 & -2.765830 \\ 138 & 1 & 0 & -6.741014 & -1.549013 & -2.713862 \\ 139 & 1 & 0 & -5.494780 & -2.316110 & -3.674557 \\ 140 & 6 & 0 & -6.720146 & -0.456091 & 0.665297 \\ 141 & 6 & 0 & -6.407818 & 0.729453 & -0.020855 \\ 142 & 6 & 0 & -6.577128 & -0.484281 & 2.056961 \\ 143 & 6 & 0 & -5.863613 & 1.794418 & 0.705242 \\ 144 & 6 & 0 & -6.057535 & 0.584815 & 2.774380 \\ 145 & 1 & 0 & -6.862224 & -1.372131 & 2.624770 \\ 146 & 6 & 0 & -5.638326 & 1.726208 & 2.074383 \\ 147 & 1 & 0 & -5.569438 & 2.715614 & 0.198160 \\ 148 & 6 & 0 & -4.929214 & -0.398687 & -2.937004 \\ 149 & 6 & 0 & -3.759868 & -0.389640 & -3.708682 \\ 150 & 6 & 0 & -5.378906 & 0.807094 & -2.373906 \\ 151 & 6 & 0 & -3.014912 & 0.767397 & -3.922299 \\ 152 & 1 & 0 & -3.424297 & -1.318569 & -4.172847 \\ 153 & 6 & 0 & -4.650921 & 1.974525 & -2.634529 \\ 154 & 6 & 0 & -3.485574 & 1.978655 & -3.388622 \\ 155 & 1 & 0 & -4.989540 & 2.934813 & -2.240060 \\ 156 & 6 & 0 & -6.625569 & 0.914152 & -1.514862 \\ 157 & 1 & 0 & -7.049282 & 1.917510 & -1.663497 \\ 158 & 1 & 0 & -7.390240 & 0.214387 & -1.870106 \\ 159 & 8 & 0 & -2.318215 & -4.723075 & -0.878343 \\ 160 & 8 & 0 & -3.658960 & -4.801420 & 1.598045 \\ 161 & 8 & 0 & -5.921279 & 0.475246 & 4.128374 \\ 162 & 8 & 0 & -5.024714 & 2.777578 & 2.698370 \\ 163 & 8 & 0 & -2.806009 & 3.150340 & -3.564022 \\ 164 & 8 & 0 & -1.876840 & 0.795955 & -4.661583\end{array}$




$\begin{array}{llllll}165 & 6 & 0 & -2.773064 & 3.659661 & -4.887867 \\ 166 & 1 & 0 & -2.215211 & 4.603873 & -4.852890 \\ 167 & 1 & 0 & -2.267077 & 2.963841 & -5.572146 \\ 168 & 1 & 0 & -3.793576 & 3.859049 & -5.255794 \\ 169 & 6 & 0 & -2.381836 & -6.139968 & -0.847546 \\ 170 & 1 & 0 & -2.763295 & -6.502706 & 0.118013 \\ 171 & 1 & 0 & -3.023594 & -6.520162 & -1.660185 \\ 172 & 1 & 0 & -1.359554 & -6.510727 & -0.995212 \\ 173 & 6 & 0 & -6.706475 & 1.369952 & 4.900514 \\ 174 & 1 & 0 & -6.446885 & 2.419315 & 4.692448 \\ 175 & 1 & 0 & -7.780364 & 1.217953 & 4.702383 \\ 176 & 1 & 0 & -6.502677 & 1.148065 & 5.955621\end{array}$

Compound $\boldsymbol{M - 8}$, conformer 4

\begin{tabular}{|c|c|c|c|c|c|}
\hline \multirow{2}{*}{$\begin{array}{l}\text { Center } \\
\text { Number }\end{array}$} & \multicolumn{2}{|l|}{ Atomic } & \multirow{2}{*}{$\begin{array}{l}\text { Atomic } \\
\text { Type }\end{array}$} & \multicolumn{2}{|c|}{ Coordinates (Angstrom } \\
\hline & Numbe & & & $X$ & $\mathrm{Z}$ \\
\hline 1 & 6 & 0 & 4.038976 & 1.813632 & -3.489743 \\
\hline 2 & 1 & 0 & 4.078171 & 1.127670 & -4.349209 \\
\hline 3 & 1 & 0 & 3.826878 & 2.825872 & -3.859518 \\
\hline 4 & 6 & 0 & 2.933685 & 1.385657 & -2.538146 \\
\hline 5 & 1 & 0 & 3.069963 & 0.338548 & -2.221806 \\
\hline 6 & 1 & 0 & 2.968440 & 2.007067 & -1.627212 \\
\hline 7 & 6 & 0 & 0.935346 & -0.101158 & 4.570199 \\
\hline 8 & 1 & 0 & 1.162010 & -0.926837 & 3.880827 \\
\hline 9 & 1 & 0 & 0.717723 & -0.543979 & 5.555210 \\
\hline 10 & 6 & 0 & -0.277032 & 0.682372 & 4.096888 \\
\hline 11 & 1 & 0 & -1.133265 & -0.012212 & 4.001616 \\
\hline 12 & 1 & 0 & -0.539763 & 1.439326 & 4.849918 \\
\hline 13 & 6 & 0 & 1.281189 & -3.884915 & -1.808525 \\
\hline 14 & 1 & 0 & 0.377989 & -4.071576 & -1.205396 \\
\hline 15 & 1 & 0 & 1.592235 & -2.839871 & -1.644056 \\
\hline 16 & 6 & 0 & 1.017912 & -4.098182 & -3.291224 \\
\hline 17 & 1 & 0 & 1.968500 & -3.992519 & -3.833811 \\
\hline 18 & 1 & 0 & 0.645293 & -5.125541 & -3.461390 \\
\hline 19 & 8 & 0 & 1.709852 & 1.537821 & -3.222811 \\
\hline 20 & 8 & 0 & -0.050831 & 1.388142 & 2.903813 \\
\hline 21 & 8 & 0 & 0.132166 & -3.147275 & -3.828497 \\
\hline 22 & 6 & 0 & -2.900044 & -2.725738 & -1.645816 \\
\hline 23 & 6 & 0 & -1.642496 & -2.287363 & -2.394952 \\
\hline 24 & 6 & 0 & -2.140660 & 0.029079 & -2.153875 \\
\hline 25 & 6 & 0 & -3.428307 & -0.277602 & -1.369654 \\
\hline 26 & 6 & 0 & -3.338783 & -1.633727 & -0.675877 \\
\hline 27 & 1 & 0 & -0.833458 & -2.187845 & -1.655011 \\
\hline 28 & 1 & 0 & -3.713227 & -2.900007 & -2.374236 \\
\hline 29 & 1 & 0 & -2.275637 & 0.911964 & -2.789533 \\
\hline 30 & 1 & 0 & -4.247691 & -0.334008 & -2.108802 \\
\hline 31 & 1 & 0 & -2.577274 & -1.573999 & 0.119997 \\
\hline
\end{tabular}




$\begin{array}{rrrrrr}32 & 8 & 0 & -1.858793 & -1.033472 & -3.026579 \\ 33 & 8 & 0 & -1.111276 & 0.230802 & -1.229728 \\ 34 & 8 & 0 & -3.715887 & 0.691751 & -0.398269 \\ 35 & 8 & 0 & -4.585809 & -1.997426 & -0.128212 \\ 36 & 8 & 0 & -2.598000 & -3.912237 & -0.947259 \\ 37 & 6 & 0 & -4.740468 & -1.775038 & 1.255114 \\ 38 & 1 & 0 & -3.991006 & -2.356101 & 1.823777 \\ 39 & 1 & 0 & -4.568773 & -0.709136 & 1.484236 \\ 40 & 6 & 0 & -3.623966 & -4.894671 & -0.931112 \\ 41 & 1 & 0 & -3.839837 & -5.224693 & -1.963339 \\ 42 & 1 & 0 & -4.544116 & -4.460033 & -0.512143 \\ 43 & 6 & 0 & -6.126995 & -2.170587 & 1.691265 \\ 44 & 6 & 0 & -7.203477 & -2.127653 & 0.802051 \\ 45 & 6 & 0 & -6.360575 & -2.550544 & 3.016308 \\ 46 & 6 & 0 & -8.488542 & -2.454535 & 1.230899 \\ 47 & 1 & 0 & -7.022852 & -1.841802 & -0.234757 \\ 48 & 6 & 0 & -7.645361 & -2.871111 & 3.447573 \\ 49 & 1 & 0 & -5.525059 & -2.598056 & 3.720000 \\ 50 & 6 & 0 & -8.714910 & -2.824625 & 2.554838 \\ 51 & 1 & 0 & -9.320391 & -2.419412 & 0.523524 \\ 52 & 1 & 0 & -7.810977 & -3.166506 & 4.486093 \\ 53 & 1 & 0 & -9.722260 & -3.080829 & 2.890234 \\ 54 & 6 & 0 & -3.167439 & -6.062565 & -0.101638 \\ 55 & 6 & 0 & -2.549344 & -7.165051 & -0.696501 \\ 56 & 6 & 0 & -3.320252 & -6.043885 & 1.288053 \\ 57 & 6 & 0 & -2.098236 & -8.232027 & 0.078323 \\ 58 & 1 & 0 & -2.425299 & -7.190450 & -1.782239 \\ 59 & 6 & 0 & -2.868887 & -7.106368 & 2.066777 \\ 60 & 1 & 0 & -3.807081 & -5.188466 & 1.763359 \\ 61 & 6 & 0 & -2.257111 & -8.204027 & 1.462192 \\ 62 & 1 & 0 & -1.622089 & -9.090087 & -0.401140 \\ 63 & 1 & 0 & -2.999087 & -7.080930 & 3.150883 \\ 64 & 1 & 0 & -1.906604 & -9.040125 & 2.071300 \\ 65 & 6 & 0 & -1.221813 & -3.282504 & -3.461888 \\ 66 & 1 & 0 & -1.427643 & -4.303004 & -3.101332 \\ 67 & 1 & 0 & -1.837901 & -3.101389 & -4.357387 \\ 68 & 6 & 0 & 0.023762 & 1.035660 & -1.523719 \\ 69 & 8 & 0 & 0.941132 & 0.771776 & -0.510785 \\ 70 & 6 & 0 & 0.643009 & 1.547472 & 0.653950 \\ 71 & 6 & 0 & -0.282092 & 2.690728 & 0.184736 \\ 72 & 6 & 0 & -0.280359 & 2.524039 & -1.332968 \\ 73 & 1 & 0 & 1.586611 & 1.978839 & 1.021985 \\ 74 & 1 & 0 & -1.306387 & 2.550688 & 0.571325 \\ 75 & 1 & 0 & 0.588621 & 3.086405 & -1.717722 \\ 76 & 6 & 0 & 0.659836 & 0.679864 & -2.868616 \\ 81 & 1 & 0 & 0.986597 & -0.374452 & -2.831787 \\ & 1 & 0 & -0.095314 & 0.764567 & -3.660316 \\ & 8 & 0 & -1.439978 & 2.913594 & -2.005292 \\ 79 & 8 & 0 & 0.190334 & 3.978160 & 0.489255 \\ 77 & 0 & 0.000222 & 4.391404 & 1.833578\end{array}$




$\begin{array}{cccccc}82 & 1 & 0 & 0.476109 & 3.671582 & 2.517811 \\ 83 & 1 & 0 & -1.079639 & 4.409260 & 2.068363 \\ 84 & 6 & 0 & 0.603660 & 5.758447 & 1.997188 \\ 85 & 6 & 0 & -0.182648 & 6.909330 & 1.914545 \\ 86 & 6 & 0 & 1.983143 & 5.892364 & 2.187660 \\ 87 & 6 & 0 & 0.394431 & 8.173643 & 2.023956 \\ 88 & 1 & 0 & -1.261495 & 6.813707 & 1.766077 \\ 89 & 6 & 0 & 2.563003 & 7.153993 & 2.294067 \\ 90 & 1 & 0 & 2.601063 & 4.993351 & 2.262347 \\ 91 & 6 & 0 & 1.769085 & 8.297852 & 2.212160 \\ 92 & 1 & 0 & -0.232652 & 9.065825 & 1.961407 \\ 93 & 1 & 0 & 3.640867 & 7.246911 & 2.445602 \\ 94 & 1 & 0 & 2.223203 & 9.287541 & 2.298517 \\ 95 & 6 & 0 & -4.433506 & 1.813050 & -0.851179 \\ 96 & 1 & 0 & -3.887604 & 2.319899 & -1.665813 \\ 97 & 1 & 0 & -5.412470 & 1.497148 & -1.259645 \\ 98 & 6 & 0 & -4.648113 & 2.794010 & 0.273277 \\ 99 & 6 & 0 & -4.078013 & 2.611668 & 1.533815 \\ 100 & 6 & 0 & -5.431000 & 3.931423 & 0.044327 \\ 101 & 6 & 0 & -4.284336 & 3.550267 & 2.546352 \\ 102 & 1 & 0 & -3.468320 & 1.727552 & 1.720117 \\ 103 & 6 & 0 & -5.635906 & 4.867709 & 1.052559 \\ 104 & 1 & 0 & -5.883690 & 4.085769 & -0.938775 \\ 105 & 6 & 0 & -5.061242 & 4.680561 & 2.310394 \\ 106 & 1 & 0 & -3.831852 & 3.392619 & 3.528140 \\ 107 & 1 & 0 & -6.250068 & 5.749635 & 0.857026 \\ 108 & 1 & 0 & -5.222822 & 5.413977 & 3.103319 \\ 109 & 6 & 0 & -2.688297 & 4.602506 & -3.142574 \\ 110 & 6 & 0 & -2.673098 & 3.937747 & -4.373117 \\ 111 & 6 & 0 & -3.674941 & 5.561277 & -2.905313 \\ 112 & 6 & 0 & -3.625523 & 4.227854 & -5.345621 \\ 113 & 1 & 0 & -1.906053 & 3.184474 & -4.563903 \\ 114 & 6 & 0 & -4.625505 & 5.859246 & -3.881176 \\ 115 & 1 & 0 & -3.703881 & 6.078368 & -1.943067 \\ 116 & 6 & 0 & -4.604259 & 5.191674 & -5.102998 \\ 117 & 1 & 0 & -3.603277 & 3.700223 & -6.301828 \\ 118 & 1 & 0 & -5.390966 & 6.612271 & -3.681096 \\ 119 & 1 & 0 & -5.351009 & 5.419898 & -5.866643 \\ 120 & 6 & 0 & -1.636744 & 4.310691 & -2.105086 \\ 121 & 1 & 0 & -0.680984 & 4.791557 & -2.387789 \\ 122 & 1 & 0 & -1.929981 & 4.735810 & -1.130961 \\ 123 & 6 & 0 & 0.073530 & 0.633451 & 1.726593 \\ 124 & 1 & 0 & 0.767078 & -0.216114 & 1.848002 \\ 125 & 1 & 0 & -0.903055 & 0.229726 & 1.403694 \\ 126 & 6 & 0 & 3.300489 & -3.644439 & 1.933943 \\ 127 & 6 & 0 & 4.600680 & -3.174120 & 1.707591 \\ 128 & 6 & 0 & 5.167624 & -3.348786 & 0.435672 \\ 129 & 6 & 0 & 4.385540 & -3.932308 & -0.568976 \\ 130 & 6 & 0 & 3.072246 & -4.322433 & -0.357223 \\ 131 & 6 & 0 & 2.524431 & -4.206607 & 0.929522\end{array}$




\begin{tabular}{|c|c|c|c|c|c|}
\hline 132 & 1 & 0 & 2.843427 & -3.552942 & 2.921049 \\
\hline 133 & 1 & 0 & 4.780749 & -4.047350 & -1.579771 \\
\hline 134 & 6 & 0 & 6.543958 & -2.830817 & 0.044739 \\
\hline 135 & 1 & 0 & 7.021544 & -3.569249 & -0.614172 \\
\hline 136 & 1 & 0 & 7.192954 & -2.735297 & 0.921473 \\
\hline 137 & 6 & 0 & 5.292074 & -2.438778 & 2.841966 \\
\hline 138 & 1 & 0 & 6.380667 & -2.479175 & 2.728120 \\
\hline 139 & 1 & 0 & 5.065698 & -2.961191 & 3.782024 \\
\hline 140 & 6 & 0 & 6.417977 & -1.517088 & -0.702448 \\
\hline 141 & 6 & 0 & 6.350517 & -0.268457 & -0.059967 \\
\hline 142 & 6 & 0 & 6.250429 & -1.567945 & -2.090291 \\
\hline 143 & 6 & 0 & 5.994753 & 0.850879 & -0.820580 \\
\hline 144 & 6 & 0 & 5.925411 & -0.447576 & -2.843297 \\
\hline 145 & 1 & 0 & 6.356137 & -2.513396 & -2.625991 \\
\hline 146 & 6 & 0 & 5.730470 & 0.774796 & -2.183438 \\
\hline 147 & 1 & 0 & 5.887183 & 1.829132 & -0.347370 \\
\hline 148 & 6 & 0 & 4.823944 & -0.998858 & 2.977702 \\
\hline 149 & 6 & 0 & 3.700764 & -0.749734 & 3.776022 \\
\hline 150 & 6 & 0 & 5.446290 & 0.080236 & 2.328559 \\
\hline 151 & 6 & 0 & 3.160860 & 0.523879 & 3.932483 \\
\hline 152 & 1 & 0 & 3.227712 & -1.582231 & 4.299295 \\
\hline 153 & 6 & 0 & 4.938803 & 1.367191 & 2.547247 \\
\hline 154 & 6 & 0 & 3.816413 & 1.609571 & 3.328394 \\
\hline 155 & 1 & 0 & 5.422497 & 2.234116 & 2.092352 \\
\hline 156 & 6 & 0 & 6.652437 & -0.073698 & 1.419501 \\
\hline 157 & 1 & 0 & 7.257701 & 0.839460 & 1.510775 \\
\hline 158 & 1 & 0 & 7.291810 & -0.888935 & 1.776702 \\
\hline 159 & 8 & 0 & 1.238220 & -4.565357 & 1.202111 \\
\hline 160 & 8 & 0 & 2.325926 & -4.767329 & -1.414227 \\
\hline 161 & 8 & 0 & 5.752108 & -0.578598 & -4.191248 \\
\hline 162 & 8 & 0 & 5.303996 & 1.896533 & -2.839878 \\
\hline 163 & 8 & 0 & 3.357269 & 2.889168 & 3.458657 \\
\hline 164 & 8 & 0 & 2.051256 & 0.770301 & 4.676496 \\
\hline 165 & 6 & 0 & 3.426031 & 3.446986 & 4.761423 \\
\hline 166 & 1 & 0 & 3.046598 & 4.474032 & 4.688739 \\
\hline 167 & 1 & 0 & 2.808196 & 2.880421 & 5.472535 \\
\hline 168 & 1 & 0 & 4.468422 & 3.473868 & 5.120838 \\
\hline 169 & 6 & 0 & 0.912200 & -5.936368 & 1.033499 \\
\hline 170 & 1 & 0 & 1.521403 & -6.567132 & 1.702242 \\
\hline 171 & 1 & 0 & -0.146174 & -6.049818 & 1.298361 \\
\hline 172 & 1 & 0 & 1.062844 & -6.262776 & -0.006299 \\
\hline 173 & 6 & 0 & 6.678588 & 0.127712 & -5.001010 \\
\hline 174 & 1 & 0 & 6.620106 & 1.213711 & -4.830040 \\
\hline 175 & 1 & 0 & 7.708534 & -0.213102 & -4.804473 \\
\hline 176 & 1 & 0 & 6.423337 & -0.089203 & -6.045917 \\
\hline
\end{tabular}

Compound $\boldsymbol{M - 8}$, conformer 5

Center Atomic Atomic Coordinates (Angstroms) 


\begin{tabular}{|c|c|c|c|c|c|}
\hline Number & \multicolumn{2}{|c|}{ Number } & Type & $X$ & $\mathrm{Z}$ \\
\hline 1 & 6 & 0 & -4.660058 & -1.273441 & 2.852401 \\
\hline 2 & 6 & 0 & -5.292521 & -0.070090 & 2.515628 \\
\hline 3 & 6 & 0 & -4.728086 & 1.130521 & 2.978046 \\
\hline 4 & 6 & 0 & -3.539810 & 1.076030 & 3.717330 \\
\hline 5 & 6 & 0 & -2.883827 & -0.120428 & 3.994379 \\
\hline 6 & 6 & 0 & -3.475624 & -1.323623 & 3.575376 \\
\hline 7 & 1 & 0 & -5.091943 & -2.227503 & 2.543075 \\
\hline 8 & 1 & 0 & -3.109838 & 2.004027 & 4.097796 \\
\hline 9 & 6 & 0 & -5.333571 & 2.499731 & 2.709167 \\
\hline 10 & 1 & 0 & -5.098355 & 3.149297 & 3.563862 \\
\hline 11 & 1 & 0 & -6.426346 & 2.433349 & 2.671811 \\
\hline 12 & 6 & 0 & -6.564521 & -0.135548 & 1.689315 \\
\hline 13 & 1 & 0 & -7.249906 & 0.665575 & 1.988455 \\
\hline 14 & 1 & 0 & -7.078055 & -1.074050 & 1.942003 \\
\hline 15 & 6 & 0 & -4.788328 & 3.150028 & 1.450087 \\
\hline 16 & 6 & 0 & -5.437349 & 3.094346 & 0.206698 \\
\hline 17 & 6 & 0 & -3.543702 & 3.789668 & 1.530398 \\
\hline 18 & 6 & 0 & -4.775462 & 3.607876 & -0.915328 \\
\hline 19 & 6 & 0 & -2.893925 & 4.297646 & 0.413751 \\
\hline 20 & 1 & 0 & -3.034450 & 3.888894 & 2.491047 \\
\hline 21 & 6 & 0 & -3.507908 & 4.163505 & -0.840869 \\
\hline 22 & 1 & 0 & -5.230835 & 3.539674 & -1.904828 \\
\hline 23 & 6 & 0 & -6.378349 & -0.115213 & 0.178647 \\
\hline 24 & 6 & 0 & -5.984148 & -1.294931 & -0.462255 \\
\hline 25 & 6 & 0 & -6.588632 & 1.031634 & -0.606387 \\
\hline 26 & 6 & 0 & -5.816233 & -1.375716 & -1.840128 \\
\hline 27 & 1 & 0 & -5.766901 & -2.193796 & 0.118568 \\
\hline 28 & 6 & 0 & -6.516764 & 0.915891 & -1.998455 \\
\hline 29 & 6 & 0 & -6.151796 & -0.265826 & -2.629526 \\
\hline 30 & 1 & 0 & -6.734392 & 1.774250 & -2.637175 \\
\hline 31 & 6 & 0 & -6.780285 & 2.415401 & -0.018128 \\
\hline 32 & 1 & 0 & -7.362118 & 3.023091 & -0.725056 \\
\hline 33 & 1 & 0 & -7.362260 & 2.381239 & 0.908636 \\
\hline 34 & 8 & 0 & -2.898801 & -2.535658 & 3.826679 \\
\hline 35 & 8 & 0 & -1.718150 & -0.180797 & 4.689588 \\
\hline 36 & 8 & 0 & -1.661393 & 4.868001 & 0.553103 \\
\hline 37 & 8 & 0 & -2.866811 & 4.531213 & -1.992688 \\
\hline 38 & 8 & 0 & -6.077099 & -0.296813 & -3.992350 \\
\hline 39 & 8 & 0 & -5.340339 & -2.537238 & -2.384132 \\
\hline 40 & 6 & 0 & -6.989815 & -1.169983 & -4.638623 \\
\hline 41 & 1 & 0 & -6.822064 & -1.068678 & -5.718252 \\
\hline 42 & 1 & 0 & -6.827351 & -2.217579 & -4.341807 \\
\hline 43 & 1 & 0 & -8.030369 & -0.887357 & -4.408573 \\
\hline 44 & 6 & 0 & -2.837065 & -2.927959 & 5.188851 \\
\hline 45 & 1 & 0 & -2.228919 & -2.228473 & 5.779699 \\
\hline 46 & 1 & 0 & -3.848764 & -2.992445 & 5.623398 \\
\hline 47 & 1 & 0 & -2.373835 & -3.922365 & 5.213296 \\
\hline 48 & 6 & 0 & -1.574594 & 6.240809 & 0.198587 \\
\hline
\end{tabular}




$\begin{array}{rrrrrr}49 & 1 & 0 & -2.261768 & 6.845066 & 0.813464 \\ 50 & 1 & 0 & -0.543320 & 6.556470 & 0.397112 \\ 51 & 1 & 0 & -1.807691 & 6.396444 & -0.865231 \\ 52 & 6 & 0 & -4.127238 & -2.440574 & -3.125358 \\ 53 & 1 & 0 & -4.273907 & -1.869144 & -4.054195 \\ 54 & 1 & 0 & -3.854816 & -3.471644 & -3.388047 \\ 55 & 6 & 0 & -3.009404 & -1.816049 & -2.306855 \\ 56 & 1 & 0 & -3.223878 & -0.755746 & -2.094943 \\ 57 & 1 & 0 & -2.929809 & -2.331195 & -1.334595 \\ 58 & 6 & 0 & -0.683446 & 0.745904 & 4.396510 \\ 59 & 1 & 0 & -1.014925 & 1.453394 & 3.623261 \\ 60 & 1 & 0 & -0.445862 & 1.331445 & 5.298944 \\ 61 & 6 & 0 & 0.559212 & -0.005880 & 3.949482 \\ 62 & 1 & 0 & 1.347706 & 0.729774 & 3.700385 \\ 63 & 1 & 0 & 0.928282 & -0.627156 & 4.778172 \\ 64 & 6 & 0 & -1.763972 & 3.695760 & -2.326106 \\ 65 & 1 & 0 & -0.876988 & 3.988705 & -1.741798 \\ 66 & 1 & 0 & -2.004807 & 2.647106 & -2.083738 \\ 67 & 6 & 0 & -1.506575 & 3.809828 & -3.819704 \\ 68 & 1 & 0 & -1.196799 & 4.841250 & -4.071417 \\ 69 & 1 & 0 & -2.444036 & 3.601213 & -4.355446 \\ 70 & 8 & 0 & -1.820110 & -1.935792 & -3.055776 \\ 71 & 8 & 0 & 0.322266 & -0.888213 & 2.882650 \\ 72 & 8 & 0 & -0.558390 & 2.874840 & -4.272191 \\ 73 & 6 & 0 & 2.493758 & 2.821993 & -2.079259 \\ 74 & 6 & 0 & 1.266568 & 2.240029 & -2.781483 \\ 75 & 6 & 0 & 1.943924 & -0.007167 & -2.381103 \\ 76 & 6 & 0 & 3.236096 & 0.446870 & -1.684379 \\ 77 & 6 & 0 & 3.056542 & 1.829480 & -1.064591 \\ 78 & 1 & 0 & 0.469651 & 2.145384 & -2.027956 \\ 79 & 1 & 0 & 3.275769 & 3.034884 & -2.831881 \\ 80 & 1 & 0 & 2.109695 & -0.931827 & -2.946218 \\ 81 & 1 & 0 & 4.014814 & 0.524571 & -2.464114 \\ 82 & 1 & 0 & 2.331609 & 1.753174 & -0.236999 \\ 83 & 8 & 0 & 1.557324 & 0.959665 & -3.322368 \\ 84 & 8 & 0 & 0.972077 & -0.187351 & -1.393152 \\ 85 & 8 & 0 & 3.644211 & -0.437220 & -0.675058 \\ 86 & 8 & 0 & 4.287280 & 2.327797 & -0.590895 \\ 87 & 8 & 0 & 2.076128 & 4.010716 & -1.450666 \\ 88 & 6 & 0 & 4.525496 & 2.180345 & 0.792454 \\ 89 & 1 & 0 & 3.781122 & 2.755401 & 1.372161 \\ 90 & 1 & 0 & 4.421117 & 1.119696 & 1.077378 \\ 91 & 6 & 0 & 3.039673 & 5.018264 & -1.226560 \\ 92 & 1 & 0 & 2.837546 & 5.860888 & -1.914134 \\ 93 & 1 & 0 & 4.048849 & 4.644616 & -1.449211 \\ 94 & 6 & 0 & 5.912711 & 2.659280 & 1.131226 \\ 96 & 6 & 0 & 6.951683 & 2.577223 & 0.200893 \\ 98 & 1 & 0 & 6.738439 & 2.199455 & -0.799801\end{array}$




$\begin{array}{cccccc}99 & 6 & 0 & 7.477563 & 3.555856 & 2.752559 \\ 100 & 1 & 0 & 5.381127 & 3.240401 & 3.140659 \\ 101 & 6 & 0 & 8.509290 & 3.469680 & 1.819317 \\ 102 & 1 & 0 & 9.042515 & 2.915197 & -0.197095 \\ 103 & 1 & 0 & 7.675881 & 3.943774 & 3.754276 \\ 104 & 1 & 0 & 9.519842 & 3.786684 & 2.086203 \\ 105 & 6 & 0 & 3.003917 & 5.521751 & 0.197355 \\ 106 & 6 & 0 & 1.944081 & 5.222703 & 1.055168 \\ 107 & 6 & 0 & 4.045187 & 6.329554 & 0.666069 \\ 108 & 6 & 0 & 1.927634 & 5.721599 & 2.358311 \\ 109 & 1 & 0 & 1.124694 & 4.597790 & 0.698634 \\ 110 & 6 & 0 & 4.028982 & 6.827924 & 1.965413 \\ 111 & 1 & 0 & 4.883548 & 6.567524 & 0.005436 \\ 112 & 6 & 0 & 2.968017 & 6.524277 & 2.818671 \\ 113 & 1 & 0 & 1.089436 & 5.480097 & 3.016248 \\ 114 & 1 & 0 & 4.852591 & 7.453605 & 2.316757 \\ 115 & 1 & 0 & 2.954463 & 6.912984 & 3.839255 \\ 116 & 6 & 0 & 0.781689 & 3.126482 & -3.916184 \\ 117 & 1 & 0 & 0.918803 & 4.181205 & -3.629365 \\ 118 & 1 & 0 & 1.413095 & 2.923157 & -4.796351 \\ 119 & 6 & 0 & -0.098083 & -1.112670 & -1.525957 \\ 120 & 8 & 0 & -0.974315 & -0.815721 & -0.486232 \\ 121 & 6 & 0 & -0.514775 & -1.405969 & 0.734137 \\ 122 & 6 & 0 & 0.474873 & -2.518729 & 0.325949 \\ 123 & 6 & 0 & 0.350617 & -2.538288 & -1.196055 \\ 124 & 1 & 0 & -1.382449 & -1.862398 & 1.234278 \\ 125 & 1 & 0 & 1.507469 & -2.249395 & 0.607205 \\ 126 & 1 & 0 & -0.489668 & -3.210868 & -1.443289 \\ 127 & 6 & 0 & -0.835282 & -0.959423 & -2.856130 \\ 128 & 1 & 0 & -1.254754 & 0.061470 & -2.903561 \\ 129 & 1 & 0 & -0.117864 & -1.062540 & -3.680425 \\ 130 & 8 & 0 & 1.489202 & -2.910147 & -1.912385 \\ 131 & 8 & 0 & 0.142727 & -3.792791 & 0.815755 \\ 132 & 6 & 0 & 0.456673 & -4.013689 & 2.181980 \\ 133 & 1 & 0 & -0.029459 & -3.247543 & 2.806501 \\ 134 & 1 & 0 & 1.547643 & -3.922521 & 2.333051 \\ 135 & 6 & 0 & -0.020348 & -5.387960 & 2.560802 \\ 136 & 6 & 0 & 0.854227 & -6.476151 & 2.579925 \\ 137 & 6 & 0 & -1.371709 & -5.599394 & 2.854918 \\ 138 & 6 & 0 & 0.391902 & -7.754150 & 2.890838 \\ 139 & 1 & 0 & 1.911784 & -6.319795 & 2.351477 \\ 140 & 6 & 0 & -1.837204 & -6.875244 & 3.162344 \\ 141 & 1 & 0 & -2.058396 & -4.748410 & 2.850521 \\ 142 & 6 & 0 & -0.955521 & -7.955947 & 3.180650 \\ 143 & 1 & 0 & 1.087519 & -8.596188 & 2.905939 \\ 144 & 1 & 0 & -2.893915 & -7.028492 & 3.393118 \\ 145 & 1 & 0 & -1.319867 & -8.956393 & 3.424610 \\ 146 & 6 & 0 & 4.453999 & -1.507155 & -1.094935 \\ 147 & 1 & 0 & 3.931075 & -2.116716 & -1.852310 \\ 148 & 1 & 0 & 5.377179 & -1.119869 & -1.567138\end{array}$




\begin{tabular}{|c|c|c|c|c|c|}
\hline 149 & 6 & 0 & 4.817795 & -2.387493 & 0.073725 \\
\hline 150 & 6 & 0 & 4.328932 & -2.152820 & 1.359402 \\
\hline 151 & 6 & 0 & 5.667177 & -3.479980 & -0.136531 \\
\hline 152 & 6 & 0 & 4.680453 & -2.995838 & 2.415139 \\
\hline 153 & 1 & 0 & 3.666448 & -1.304619 & 1.531763 \\
\hline 154 & 6 & 0 & 6.017369 & -4.320725 & 0.914894 \\
\hline 155 & 1 & 0 & 6.056567 & -3.675552 & -1.139195 \\
\hline 156 & 6 & 0 & 5.523553 & -4.081562 & 2.198022 \\
\hline 157 & 1 & 0 & 4.289865 & -2.798161 & 3.416070 \\
\hline 158 & 1 & 0 & 6.681557 & -5.168677 & 0.733116 \\
\hline 159 & 1 & 0 & 5.798860 & -4.739928 & 3.024756 \\
\hline 160 & 6 & 0 & 2.779875 & -4.626038 & -2.962614 \\
\hline 161 & 6 & 0 & 2.627610 & -4.081892 & -4.241796 \\
\hline 162 & 6 & 0 & 3.828921 & -5.517369 & -2.728604 \\
\hline 163 & 6 & 0 & 3.507033 & -4.423955 & -5.265255 \\
\hline 164 & 1 & 0 & 1.811390 & -3.381958 & -4.430503 \\
\hline 165 & 6 & 0 & 4.705902 & -5.867658 & -3.754308 \\
\hline 166 & 1 & 0 & 3.965116 & -5.939497 & -1.729891 \\
\hline 167 & 6 & 0 & 4.548289 & -5.320110 & -5.025100 \\
\hline 168 & 1 & 0 & 3.377792 & -3.990065 & -6.259385 \\
\hline 169 & 1 & 0 & 5.521345 & -6.566728 & -3.555847 \\
\hline 170 & 1 & 0 & 5.237569 & -5.589004 & -5.828507 \\
\hline 171 & 6 & 0 & 1.805449 & -4.287243 & -1.865396 \\
\hline 172 & 1 & 0 & 0.877006 & -4.876624 & -1.989241 \\
\hline 173 & 1 & 0 & 2.224604 & -4.559849 & -0.882525 \\
\hline 174 & 6 & 0 & 0.046990 & -0.314619 & 1.631123 \\
\hline 175 & 1 & 0 & -0.709927 & 0.485356 & 1.697824 \\
\hline 176 & 1 & 0 & 0.957998 & 0.117558 & 1.180351 \\
\hline
\end{tabular}

Compound $\boldsymbol{M - 8}$, conformer 6

\begin{tabular}{cccccc} 
Center & \multicolumn{2}{c}{ Atomic } & \multicolumn{2}{c}{ Atomic } & \multicolumn{3}{c}{ Coordinates } \\
Number & Number & Type & X & Y & Z \\
- & Nums & \\
1 & 6 & 0 & 0.134797 & -4.404015 & 3.341042 \\
2 & 1 & 0 & 0.678850 & -4.144646 & 4.261765 \\
3 & 1 & 0 & -0.883885 & -4.712379 & 3.612071 \\
4 & 6 & 0 & 0.064351 & -3.191007 & 2.427700 \\
5 & 1 & 0 & 1.072848 & -2.802414 & 2.208208 \\
6 & 1 & 0 & -0.391275 & -3.476738 & 1.463905 \\
7 & 6 & 0 & 0.933055 & -0.340542 & -4.465530 \\
8 & 1 & 0 & 1.667209 & -0.146576 & -3.670613 \\
9 & 1 & 0 & 1.322597 & 0.112520 & -5.391263 \\
10 & 6 & 0 & -0.410913 & 0.285102 & -4.131918 \\
11 & 1 & 0 & -0.266197 & 1.370656 & -3.974375 \\
12 & 1 & 0 & -1.094823 & 0.157012 & -4.983123 \\
13 & 6 & 0 & 4.701709 & 0.456170 & 2.943033 \\
14 & 1 & 0 & 4.143382 & -0.491294 & 3.017575 \\
15 & 1 & 0 & 5.125396 & 0.680221 & 3.932453
\end{tabular}




\begin{tabular}{|c|c|c|c|c|c|}
\hline 16 & 6 & 0 & 3.774285 & 1.583897 & 2.527085 \\
\hline 17 & 1 & 0 & 3.368195 & 1.393672 & 1.517983 \\
\hline 18 & 1 & 0 & 4.352146 & 2.525529 & 2.471253 \\
\hline 19 & 8 & 0 & -0.701739 & -2.216465 & 3.098266 \\
\hline 20 & 8 & 0 & -1.045784 & -0.314928 & -3.031807 \\
\hline 21 & 8 & 0 & 2.756941 & 1.685214 & 3.493090 \\
\hline 22 & 6 & 0 & 0.448397 & 4.070735 & 1.764199 \\
\hline 23 & 6 & 0 & 0.843892 & 2.724477 & 2.371650 \\
\hline 24 & 6 & 0 & -1.358318 & 1.844208 & 2.191618 \\
\hline 25 & 6 & 0 & -1.873629 & 3.130616 & 1.525012 \\
\hline 26 & 6 & 0 & -0.753724 & 3.911662 & 0.837540 \\
\hline 27 & 1 & 0 & 1.171028 & 2.066650 & 1.549370 \\
\hline 28 & 1 & 0 & 0.167288 & 4.759894 & 2.581568 \\
\hline 29 & 1 & 0 & -2.135598 & 1.422176 & 2.840548 \\
\hline 30 & 1 & 0 & -2.261765 & 3.772028 & 2.336276 \\
\hline 31 & 1 & 0 & -0.417826 & 3.353139 & -0.052813 \\
\hline 32 & 8 & 0 & -0.275301 & 2.145764 & 3.030122 \\
\hline 33 & 8 & 0 & -0.993697 & 0.934376 & 1.196582 \\
\hline 34 & 8 & 0 & -2.883128 & 2.874788 & 0.584798 \\
\hline 35 & 8 & 0 & -1.197049 & 5.202799 & 0.480015 \\
\hline 36 & 8 & 0 & 1.556483 & 4.572496 & 1.056520 \\
\hline 37 & 6 & 0 & -1.756726 & 5.365465 & -0.816583 \\
\hline 38 & 1 & 0 & -2.439842 & 4.535626 & -1.041364 \\
\hline 39 & 1 & 0 & -2.353423 & 6.287538 & -0.757470 \\
\hline 40 & 6 & 0 & 1.807340 & 5.949484 & 1.202741 \\
\hline 41 & 1 & 0 & 2.015778 & 6.187924 & 2.263986 \\
\hline 42 & 1 & 0 & 0.916036 & 6.528394 & 0.910396 \\
\hline 43 & 6 & 0 & -0.720492 & 5.503633 & -1.906577 \\
\hline 44 & 6 & 0 & -0.491034 & 4.469006 & -2.816557 \\
\hline 45 & 6 & 0 & 0.032371 & 6.677454 & -2.022771 \\
\hline 46 & 6 & 0 & 0.470372 & 4.599119 & -3.818663 \\
\hline 47 & 1 & 0 & -1.080776 & 3.551275 & -2.745521 \\
\hline 48 & 6 & 0 & 0.992239 & 6.812558 & -3.020663 \\
\hline 49 & 1 & 0 & -0.143928 & 7.499621 & -1.324275 \\
\hline 50 & 6 & 0 & 1.214893 & 5.770831 & -3.921526 \\
\hline 51 & 1 & 0 & 0.636199 & 3.782030 & -4.524510 \\
\hline 52 & 1 & 0 & 1.570688 & 7.735747 & -3.098589 \\
\hline 53 & 1 & 0 & 1.967041 & 5.876178 & -4.706431 \\
\hline 54 & 6 & 0 & 2.982973 & 6.367351 & 0.358194 \\
\hline 55 & 6 & 0 & 3.658121 & 5.466259 & -0.465142 \\
\hline 56 & 6 & 0 & 3.401254 & 7.702251 & 0.387518 \\
\hline 57 & 6 & 0 & 4.728569 & 5.895249 & -1.251292 \\
\hline 58 & 1 & 0 & 3.336676 & 4.424909 & -0.488920 \\
\hline 59 & 6 & 0 & 4.470159 & 8.129342 & -0.393704 \\
\hline 60 & 1 & 0 & 2.879824 & 8.417449 & 1.030026 \\
\hline 61 & 6 & 0 & 5.138609 & 7.224988 & -1.219508 \\
\hline 62 & 1 & 0 & 5.243556 & 5.181797 & -1.899018 \\
\hline 63 & 1 & 0 & 4.783947 & 9.175104 & -0.359336 \\
\hline 64 & 1 & 0 & 5.976298 & 7.558399 & -1.835870 \\
\hline 65 & 6 & 0 & 1.969799 & 2.846421 & 3.386212 \\
\hline
\end{tabular}




$\begin{array}{cccccc}66 & 1 & 0 & 2.604669 & 3.707833 & 3.119869 \\ 67 & 1 & 0 & 1.521016 & 3.032497 & 4.373330 \\ 68 & 6 & 0 & -1.046266 & -0.470114 & 1.421865 \\ 69 & 8 & 0 & -0.276562 & -1.045141 & 0.415144 \\ 70 & 6 & 0 & -1.027507 & -1.118591 & -0.801784 \\ 71 & 6 & 0 & -2.510465 & -0.954943 & -0.398681 \\ 72 & 6 & 0 & -2.446203 & -1.009202 & 1.126288 \\ 73 & 1 & 0 & -0.881902 & -2.122266 & -1.230435 \\ 74 & 1 & 0 & -2.897605 & 0.029067 & -0.714733 \\ 75 & 1 & 0 & -2.459664 & -2.075228 & 1.412722 \\ 76 & 6 & 0 & -0.463847 & -0.873870 & 2.777400 \\ 77 & 1 & 0 & 0.615162 & -0.636769 & 2.770572 \\ 78 & 1 & 0 & -0.930491 & -0.278525 & 3.571151 \\ 79 & 8 & 0 & -3.431760 & -0.313888 & 1.826696 \\ 80 & 8 & 0 & -3.349180 & -1.987236 & -0.847655 \\ 81 & 6 & 0 & -3.721744 & -1.908528 & -2.214551 \\ 82 & 1 & 0 & -2.819681 & -1.873746 & -2.845929 \\ 83 & 1 & 0 & -4.289184 & -0.976963 & -2.392824 \\ 84 & 6 & 0 & -4.558126 & -3.112117 & -2.548520 \\ 85 & 6 & 0 & -5.951988 & -3.037090 & -2.577922 \\ 86 & 6 & 0 & -3.940169 & -4.343974 & -2.789821 \\ 87 & 6 & 0 & -6.719054 & -4.169529 & -2.848251 \\ 88 & 1 & 0 & -6.442817 & -2.078568 & -2.389730 \\ 89 & 6 & 0 & -4.703803 & -5.477443 & -3.056361 \\ 90 & 1 & 0 & -2.848483 & -4.405987 & -2.775969 \\ 91 & 6 & 0 & -6.095798 & -5.392259 & -3.086175 \\ 92 & 1 & 0 & -7.808637 & -4.096241 & -2.872070 \\ 93 & 1 & 0 & -4.210998 & -6.433773 & -3.246059 \\ 94 & 1 & 0 & -6.694826 & -6.280717 & -3.298234 \\ 95 & 6 & 0 & -4.194729 & 2.838639 & 1.090454 \\ 96 & 1 & 0 & -4.291126 & 2.057268 & 1.863837 \\ 97 & 1 & 0 & -4.443276 & 3.805685 & 1.567985 \\ 98 & 6 & 0 & -5.179361 & 2.554872 & -0.015426 \\ 99 & 6 & 0 & -4.768128 & 2.282928 & -1.320894 \\ 100 & 6 & 0 & -6.548543 & 2.553715 & 0.274878 \\ 101 & 6 & 0 & -5.707334 & 2.011378 & -2.317305 \\ 102 & 1 & 0 & -3.703475 & 2.285776 & -1.554701 \\ 103 & 6 & 0 & -7.485090 & 2.282136 & -0.717222 \\ 104 & 1 & 0 & -6.884470 & 2.765615 & 1.293401 \\ 105 & 6 & 0 & -7.067002 & 2.008112 & -2.020152 \\ 106 & 1 & 0 & -5.369266 & 1.802113 & -3.334888 \\ 107 & 1 & 0 & -8.549857 & 2.285487 & -0.473328 \\ 108 & 1 & 0 & -7.801469 & 1.796607 & -2.800261 \\ 109 & 6 & 0 & -5.522965 & -0.390965 & 2.984909 \\ 110 & 6 & 0 & -4.931014 & -0.202025 & 4.237609 \\ 111 & 6 & 0 & -6.885842 & -0.126717 & 2.835270 \\ 112 & 6 & 0 & -5.688230 & 0.241884 & 5.318125 \\ 115 & 1 & 0 & -3.865330 & -0.404728 & 4.359275 \\ & 6 & 0 & -7.647796 & 0.310109 & 3.918051 \\ & & 0 & -7.356517 & -0.258918 & 1.857990\end{array}$




$\begin{array}{rrrrrr}116 & 6 & 0 & -7.050273 & 0.497695 & 5.162044 \\ 117 & 1 & 0 & -5.212816 & 0.387127 & 6.290837 \\ 118 & 1 & 0 & -8.712975 & 0.512324 & 3.785509 \\ 119 & 1 & 0 & -7.644408 & 0.845286 & 6.010026 \\ 120 & 6 & 0 & -4.712576 & -0.912310 & 1.826870 \\ 121 & 1 & 0 & -4.600732 & -2.009621 & 1.911219 \\ 122 & 1 & 0 & -5.235210 & -0.719483 & 0.875016 \\ 123 & 6 & 0 & -0.470676 & -0.089418 & -1.771405 \\ 124 & 1 & 0 & 0.624960 & -0.215508 & -1.788078 \\ 125 & 1 & 0 & -0.685115 & 0.932060 & -1.408992 \\ 126 & 6 & 0 & 5.104844 & -0.838981 & -1.361355 \\ 127 & 6 & 0 & 5.226513 & -2.228183 & -1.228904 \\ 128 & 6 & 0 & 5.585958 & -2.755094 & 0.022901 \\ 129 & 6 & 0 & 5.777223 & -1.871133 & 1.091282 \\ 130 & 6 & 0 & 5.607188 & -0.499493 & 0.963527 \\ 131 & 6 & 0 & 5.280211 & 0.031345 & -0.292554 \\ 132 & 1 & 0 & 4.852509 & -0.396935 & -2.327345 \\ 133 & 1 & 0 & 6.060156 & -2.253032 & 2.074353 \\ 134 & 6 & 0 & 5.709319 & -4.243457 & 0.309133 \\ 135 & 1 & 0 & 6.529096 & -4.393704 & 1.025086 \\ 136 & 1 & 0 & 5.986106 & -4.796692 & -0.594407 \\ 137 & 6 & 0 & 4.959750 & -3.086497 & -2.454045 \\ 138 & 1 & 0 & 5.507575 & -4.032618 & -2.382294 \\ 139 & 1 & 0 & 5.376192 & -2.565456 & -3.327474 \\ 140 & 6 & 0 & 4.425966 & -4.776554 & 0.915752 \\ 141 & 6 & 0 & 3.355881 & -5.265425 & 0.147804 \\ 142 & 6 & 0 & 4.269173 & -4.679827 & 2.302702 \\ 143 & 6 & 0 & 2.140930 & -5.526181 & 0.790987 \\ 144 & 6 & 0 & 3.068190 & -4.964924 & 2.937922 \\ 145 & 1 & 0 & 5.100000 & -4.356067 & 2.932651 \\ 146 & 6 & 0 & 1.964441 & -5.337910 & 2.156251 \\ 147 & 1 & 0 & 1.274168 & -5.864228 & 0.219207 \\ 148 & 6 & 0 & 3.489834 & -3.356478 & -2.733403 \\ 149 & 6 & 0 & 2.797811 & -2.447604 & -3.544113 \\ 150 & 6 & 0 & 2.806123 & -4.480256 & -2.239360 \\ 151 & 6 & 0 & 1.452387 & -2.607733 & -3.866658 \\ 152 & 1 & 0 & 3.333010 & -1.589332 & -3.954312 \\ 153 & 6 & 0 & 1.467326 & -4.658936 & -2.608387 \\ 154 & 6 & 0 & 0.781432 & -3.752065 & -3.405081 \\ 155 & 1 & 0 & 0.916118 & -5.539236 & -2.271157 \\ 156 & 6 & 0 & 3.451135 & -5.525604 & -1.348022 \\ 157 & 1 & 0 & 2.954098 & -6.485836 & -1.545888 \\ 158 & 1 & 0 & 4.498767 & -5.670751 & -1.634741 \\ 159 & 8 & 0 & 5.092968 & 1.369855 & -0.476365 \\ 160 & 8 & 0 & 5.790995 & 0.324767 & 2.040986 \\ 161 & 8 & 0 & 2.980997 & -4.822010 & 4.292789 \\ 162 & 8 & 0 & 0.722614 & -5.529466 & 2.696852 \\ 163 & 8 & 0 & -0.534648 & -3.976649 & -3.693322 \\ 164 & 8 & 0 & 0.766966 & -1.737041 & -4.651457 \\ 165 & 6 & 0 & -0.838289 & -4.249570 & -5.052113\end{array}$




$\begin{array}{lllrrr}166 & 1 & 0 & -1.918993 & -4.431841 & -5.106913 \\ 167 & 1 & 0 & -0.579659 & -3.399052 & -5.698664 \\ 168 & 1 & 0 & -0.304467 & -5.150201 & -5.399113 \\ 169 & 6 & 0 & 6.249442 & 2.181983 & -0.346857 \\ 170 & 1 & 0 & 6.709374 & 2.067882 & 0.645280 \\ 171 & 1 & 0 & 6.990524 & 1.933800 & -1.125163 \\ 172 & 1 & 0 & 5.927569 & 3.222499 & -0.477255 \\ 173 & 6 & 0 & 2.727216 & -6.012147 & 5.022416 \\ 174 & 1 & 0 & 1.761840 & -6.460876 & 4.742113 \\ 175 & 1 & 0 & 3.529415 & -6.750219 & 4.857229 \\ 176 & 1 & 0 & 2.705624 & -5.739315 & 6.085004\end{array}$


Table S2. Calculated at CAM-B3LYP/SVP/PCM $\left(\mathrm{CH}_{3} \mathrm{CN}\right)$ level of theory relative energies and conformer distribution at $25^{\circ} \mathrm{C}$ for $\boldsymbol{P}-8$.

\begin{tabular}{|c|c|c|}
\hline Conformer & $\begin{array}{c}\Delta G \\
{\left[\mathrm{kcal} \mathrm{mol}^{-1}\right]}\end{array}$ & $\begin{array}{c}\text { Pop. } \\
{[\%]}\end{array}$ \\
\hline $\boldsymbol{P - 8}(1)$ & 0.00 & 52.7 \\
\hline $\boldsymbol{P - 8}(2)$ & 0.38 & 28.0 \\
\hline $\boldsymbol{P}-8(3)$ & 1.17 & 7.3 \\
\hline $\boldsymbol{P}-8(4)$ & 1.27 & 6.2 \\
\hline $\boldsymbol{P - 8}(5)$ & 1.69 & 3.1 \\
\hline $\boldsymbol{P}-8(6)$ & 1.73 & 2.7 \\
\hline
\end{tabular}

\section{Cartesian coordinates for individual conformers of compound $\boldsymbol{P}-8$.}

Compound $\boldsymbol{P}-\mathbf{8}$, conformer 1

\begin{tabular}{cccccc} 
Center & Atomic & \multicolumn{2}{c}{ Atomic } & \multicolumn{2}{c}{ Coordinates } \\
Number & Number & Type & X & Y & Z \\
\hline 1 & 8 & 0 & -3.775853 & -0.352682 & 4.666314 \\
2 & 6 & 0 & 1.271063 & 4.526839 & 2.002037 \\
3 & 1 & 0 & 2.320184 & 4.816751 & 2.149278 \\
4 & 1 & 0 & 0.773500 & 4.555417 & 2.986014 \\
5 & 6 & 0 & 1.206385 & 3.126222 & 1.417288 \\
6 & 1 & 0 & 0.162404 & 2.776913 & 1.363632 \\
7 & 1 & 0 & 1.591161 & 3.149010 & 0.382966 \\
8 & 6 & 0 & -2.548915 & -1.070968 & 4.651204 \\
9 & 1 & 0 & -2.255197 & -1.306527 & 5.686447 \\
10 & 1 & 0 & -1.747803 & -0.456058 & 4.216946 \\
11 & 6 & 0 & -2.717988 & -2.362020 & 3.878419 \\
12 & 1 & 0 & -1.792720 & -2.960520 & 3.989249 \\
13 & 1 & 0 & -3.547698 & -2.938246 & 4.313787 \\
14 & 6 & 0 & -2.847029 & 0.530449 & -3.810268 \\
15 & 1 & 0 & -2.582311 & 0.693196 & -4.864837 \\
16 & 1 & 0 & -2.250198 & 1.223848 & -3.195488 \\
17 & 6 & 0 & -2.555989 & -0.897364 & -3.387874 \\
18 & 1 & 0 & -3.025305 & -1.087945 & -2.414779 \\
19 & 1 & 0 & -2.992413 & -1.605884 & -4.117115 \\
20 & 8 & 0 & 1.970797 & 2.276419 & 2.239702 \\
21 & 8 & 0 & -3.033585 & -2.179637 & 2.520873 \\
22 & 8 & 0 & -1.156781 & -1.067978 & -3.316952 \\
23 & 6 & 0 & 3.237587 & 0.267923 & 0.537699 \\
24 & 6 & 0 & 1.804149 & 0.121088 & 1.051885 \\
25 & 6 & 0 & 0.943578 & -0.148346 & -1.150854 \\
26 & 6 & 0 & 2.316506 & 0.117693 & -1.784044 \\
27 & 6 & 0 & 3.418615 & -0.352429 & -0.842234 \\
28 & 1 & 0 & 1.630659 & -0.940624 & 1.297178 \\
29 & 1 & 0 & 3.470664 & 1.342691 & 0.459083 \\
30 & 1 & 0 & 0.133929 & 0.253694 & -1.770129 \\
& & & & &
\end{tabular}




$\begin{array}{llllll}31 & 1 & 0 & 2.406808 & 1.212600 & -1.888422 \\ 32 & 1 & 0 & 3.353616 & -1.448778 & -0.744828 \\ 33 & 8 & 0 & 0.867214 & 0.534274 & 0.069655 \\ 34 & 8 & 0 & 0.787987 & -1.534329 & -0.977389 \\ 35 & 8 & 0 & 2.490714 & -0.508048 & -3.028192 \\ 36 & 8 & 0 & 4.696507 & 0.014077 & -1.312924 \\ 37 & 8 & 0 & 4.095061 & -0.349985 & 1.471472 \\ 38 & 6 & 0 & 1.850032 & 0.103482 & -4.141024 \\ 39 & 1 & 0 & 2.124407 & -0.527614 & -4.998993 \\ 40 & 6 & 0 & 5.403643 & -0.991635 & -1.999151 \\ 41 & 1 & 0 & 5.590231 & -1.853151 & -1.330718 \\ 42 & 1 & 0 & 4.799782 & -1.362472 & -2.845349 \\ 43 & 6 & 0 & 5.249745 & 0.390779 & 1.835073 \\ 44 & 1 & 0 & 4.942997 & 1.358076 & 2.270834 \\ 45 & 1 & 0 & 5.859905 & 0.592272 & 0.940650 \\ 46 & 6 & 0 & 6.716798 & -0.455622 & -2.507750 \\ 47 & 6 & 0 & 7.746942 & -1.343067 & -2.835674 \\ 48 & 6 & 0 & 6.921486 & 0.913200 & -2.694473 \\ 49 & 6 & 0 & 8.952717 & -0.873826 & -3.349189 \\ 50 & 1 & 0 & 7.603223 & -2.416528 & -2.684819 \\ 51 & 6 & 0 & 8.131264 & 1.384572 & -3.203383 \\ 52 & 1 & 0 & 6.123466 & 1.608174 & -2.432390 \\ 53 & 6 & 0 & 9.149441 & 0.494205 & -3.534901 \\ 54 & 1 & 0 & 9.747122 & -1.580431 & -3.599940 \\ 55 & 1 & 0 & 8.278054 & 2.458458 & -3.340841 \\ 56 & 1 & 0 & 10.096706 & 0.864190 & -3.933247 \\ 57 & 6 & 0 & 6.032029 & -0.409198 & 2.839313 \\ 58 & 6 & 0 & 5.757140 & -0.300215 & 4.205107 \\ 59 & 6 & 0 & 7.003542 & -1.320762 & 2.417430 \\ 60 & 6 & 0 & 6.440493 & -1.083698 & 5.132413 \\ 61 & 1 & 0 & 4.999223 & 0.410187 & 4.545479 \\ 62 & 6 & 0 & 7.689271 & -2.106664 & 3.340722 \\ 63 & 1 & 0 & 7.226441 & -1.412587 & 1.351173 \\ 64 & 6 & 0 & 7.407826 & -1.989942 & 4.700906 \\ 65 & 1 & 0 & 6.218616 & -0.985569 & 6.197347 \\ 66 & 1 & 0 & 8.448490 & -2.812882 & 2.997549 \\ 67 & 1 & 0 & 7.946176 & -2.603677 & 5.426494 \\ 68 & 6 & 0 & 1.559848 & 0.933053 & 2.313417 \\ 69 & 1 & 0 & 2.138143 & 0.478792 & 3.129054 \\ 70 & 1 & 0 & 0.485645 & 0.866689 & 2.566865 \\ 71 & 6 & 0 & -0.475506 & -2.102877 & -1.197153 \\ 72 & 8 & 0 & -1.480740 & -1.304749 & -0.614516 \\ 73 & 6 & 0 & -2.304548 & -2.033540 & 0.286776 \\ 74 & 6 & 0 & -2.010525 & -3.508317 & 0.000663 \\ 75 & 6 & 0 & -0.553714 & -3.474280 & -0.471296 \\ 80 & 1 & 0 & -3.354108 & -1.787692 & 0.065574 \\ & 1 & 0 & -2.107827 & -4.125530 & 0.908610 \\ 79 & 1 & 0 & -0.355949 & -4.307441 & -1.166126 \\ 76 & 0 & -0.750635 & -2.265551 & -2.701985 \\ 73 & 0 & 0.183644 & -2.598227 & -3.177819\end{array}$




$\begin{array}{cccccc}81 & 1 & 0 & -1.514848 & -3.046001 & -2.840049 \\ 82 & 8 & 0 & 0.271141 & -3.559465 & 0.655279 \\ 83 & 8 & 0 & -2.793773 & -4.033875 & -1.043314 \\ 84 & 6 & 0 & -2.016643 & -1.626046 & 1.722674 \\ 85 & 1 & 0 & -2.002889 & -0.522837 & 1.775830 \\ 86 & 1 & 0 & -1.023792 & -1.999381 & 2.027845 \\ 87 & 6 & 0 & -4.150741 & -4.252998 & -0.731234 \\ 88 & 1 & 0 & -4.716469 & -3.304822 & -0.738911 \\ 89 & 1 & 0 & -4.230447 & -4.669031 & 0.291986 \\ 90 & 1 & 0 & 0.755059 & 0.054109 & -4.034093 \\ 91 & 6 & 0 & 2.286229 & 1.528256 & -4.390710 \\ 92 & 6 & 0 & 3.629217 & 1.823787 & -4.650713 \\ 93 & 6 & 0 & 1.363072 & 2.574574 & -4.352596 \\ 94 & 6 & 0 & 4.036729 & 3.135771 & -4.870015 \\ 95 & 1 & 0 & 4.362853 & 1.014390 & -4.676688 \\ 96 & 6 & 0 & 1.766567 & 3.891443 & -4.573737 \\ 97 & 1 & 0 & 0.313206 & 2.358232 & -4.138839 \\ 98 & 6 & 0 & 3.104620 & 4.174292 & -4.833156 \\ 99 & 1 & 0 & 5.088242 & 3.351632 & -5.072654 \\ 100 & 1 & 0 & 1.030492 & 4.696826 & -4.530961 \\ 101 & 1 & 0 & 3.424812 & 5.204324 & -5.005305 \\ 102 & 6 & 0 & 2.201101 & -4.391430 & 1.770874 \\ 103 & 6 & 0 & 3.225034 & -3.588675 & 2.277980 \\ 104 & 6 & 0 & 1.746290 & -5.471857 & 2.534879 \\ 105 & 6 & 0 & 3.792733 & -3.865700 & 3.522259 \\ 106 & 1 & 0 & 3.581598 & -2.726623 & 1.709718 \\ 107 & 6 & 0 & 2.307386 & -5.748099 & 3.777752 \\ 108 & 1 & 0 & 0.944108 & -6.104706 & 2.146459 \\ 109 & 6 & 0 & 3.335418 & -4.945075 & 4.273416 \\ 110 & 1 & 0 & 4.593835 & -3.228206 & 3.903944 \\ 111 & 1 & 0 & 1.945561 & -6.596799 & 4.362590 \\ 112 & 1 & 0 & 3.779244 & -5.163277 & 5.247405 \\ 113 & 6 & 0 & 1.567402 & -4.094693 & 0.438829 \\ 114 & 1 & 0 & 2.182716 & -3.387002 & -0.135823 \\ 115 & 1 & 0 & 1.477028 & -5.023784 & -0.153353 \\ 116 & 6 & 0 & -4.767182 & -5.214178 & -1.716337 \\ 117 & 6 & 0 & -3.987685 & -6.131559 & -2.423241 \\ 118 & 6 & 0 & -6.152962 & -5.218736 & -1.903211 \\ 119 & 6 & 0 & -4.582855 & -7.036136 & -3.301070 \\ 120 & 1 & 0 & -2.906192 & -6.127323 & -2.283720 \\ 121 & 6 & 0 & -6.749322 & -6.126653 & -2.774572 \\ 122 & 1 & 0 & -6.773882 & -4.502614 & -1.358102 \\ 123 & 6 & 0 & -5.964478 & -7.038579 & -3.478660 \\ 124 & 1 & 0 & -3.960245 & -7.746063 & -3.850479 \\ 125 & 1 & 0 & -7.833235 & -6.117672 & -2.909164 \\ 126 & 1 & 0 & -6.429756 & -7.747703 & -4.166738 \\ 128 & 6 & 0 & -1.613296 & 5.240706 & 1.672895 \\ 129 & 6 & 0 & -2.943792 & 5.013337 & 1.300696 \\ & 6 & 0 & -3.250185 & 4.894995 & -0.064635 \\ & & & -2.215873 & 5.041764 & -0.997088\end{array}$




\begin{tabular}{|c|c|c|c|c|c|}
\hline 131 & 6 & 0 & -0.892657 & 5.238106 & -0.622910 \\
\hline 132 & 6 & 0 & -0.584371 & 5.321356 & 0.743461 \\
\hline 133 & 1 & 0 & -1.361554 & 5.341274 & 2.730029 \\
\hline 134 & 1 & 0 & -2.424710 & 4.979020 & -2.066889 \\
\hline 135 & 6 & 0 & -4.642317 & 4.585958 & -0.587040 \\
\hline 136 & 1 & 0 & -4.779741 & 5.129489 & -1.532204 \\
\hline 137 & 1 & 0 & -5.405443 & 4.973989 & 0.096348 \\
\hline 138 & 6 & 0 & -3.973210 & 4.865178 & 2.408675 \\
\hline 139 & 1 & 0 & -4.967837 & 5.153579 & 2.052167 \\
\hline 140 & 1 & 0 & -3.720176 & 5.576807 & 3.207145 \\
\hline 141 & 6 & 0 & -4.877958 & 3.109279 & -0.859571 \\
\hline 142 & 6 & 0 & -5.456084 & 2.229089 & 0.072911 \\
\hline 143 & 6 & 0 & -4.489785 & 2.601161 & -2.103450 \\
\hline 144 & 6 & 0 & -5.641959 & 0.891906 & -0.294414 \\
\hline 145 & 6 & 0 & -4.639190 & 1.262300 & -2.446276 \\
\hline 146 & 1 & 0 & -4.050561 & 3.263667 & -2.852448 \\
\hline 147 & 6 & 0 & -5.241214 & 0.391062 & -1.528018 \\
\hline 148 & 1 & 0 & -6.115758 & 0.192223 & 0.397009 \\
\hline 149 & 6 & 0 & -3.999433 & 3.469030 & 3.004784 \\
\hline 150 & 6 & 0 & -3.090672 & 3.190200 & 4.038942 \\
\hline 151 & 6 & 0 & -4.847191 & 2.452377 & 2.547633 \\
\hline 152 & 6 & 0 & -2.985149 & 1.927768 & 4.609841 \\
\hline 153 & 1 & 0 & -2.463295 & 3.998357 & 4.412914 \\
\hline 154 & 6 & 0 & -4.722784 & 1.177432 & 3.120280 \\
\hline 155 & 6 & 0 & -3.806994 & 0.893296 & 4.119172 \\
\hline 156 & 1 & 0 & -5.355642 & 0.354012 & 2.783982 \\
\hline 157 & 6 & 0 & -5.893736 & 2.653689 & 1.463416 \\
\hline 158 & 1 & 0 & -6.775853 & 2.056824 & 1.734809 \\
\hline 159 & 1 & 0 & -6.231084 & 3.696120 & 1.452919 \\
\hline 160 & 8 & 0 & 0.722056 & 5.485415 & 1.111031 \\
\hline 161 & 8 & 0 & 0.071580 & 5.284443 & -1.585060 \\
\hline 162 & 6 & 0 & 0.795573 & 6.497750 & -1.703693 \\
\hline 163 & 1 & 0 & 1.505537 & 6.365203 & -2.529931 \\
\hline 164 & 1 & 0 & 0.119938 & 7.336089 & -1.944033 \\
\hline 165 & 1 & 0 & 1.349109 & 6.728126 & -0.782512 \\
\hline 166 & 8 & 0 & -4.233081 & 0.819756 & -3.678103 \\
\hline 167 & 8 & 0 & -5.407455 & -0.938989 & -1.789489 \\
\hline 168 & 6 & 0 & -6.310215 & -1.266248 & -2.835719 \\
\hline 169 & 1 & 0 & -6.321732 & -2.360314 & -2.917055 \\
\hline 170 & 1 & 0 & -7.327163 & -0.912776 & -2.597232 \\
\hline 171 & 1 & 0 & -5.986270 & -0.831738 & -3.791982 \\
\hline 172 & 8 & 0 & -2.151919 & 1.605210 & 5.625170 \\
\hline 173 & 6 & 0 & -1.260882 & 2.580190 & 6.114450 \\
\hline 174 & 1 & 0 & -0.672868 & 2.100450 & 6.905883 \\
\hline 175 & 1 & 0 & -0.578177 & 2.937261 & 5.325430 \\
\hline 176 & 1 & 0 & -1.797173 & 3.443795 & 6.540805 \\
\hline
\end{tabular}

Compound $\boldsymbol{P} \mathbf{- 8}$, conformer 2 


\begin{tabular}{|c|c|c|c|c|c|}
\hline \multirow{2}{*}{$\begin{array}{l}\text { Center } \\
\text { Number }\end{array}$} & Atomic & \multirow{2}{*}{\multicolumn{2}{|c|}{$\begin{array}{r}\text { Atomic } \\
\text { Type }\end{array}$}} & \multicolumn{2}{|c|}{ Coordinates (Angstrom } \\
\hline & Numbe & & & $\mathrm{X}$ & $\mathrm{Z}$ \\
\hline 1 & 6 & 0 & -4.477254 & -2.852515 & -2.202090 \\
\hline 2 & 6 & 0 & -4.900208 & -3.399695 & -0.981080 \\
\hline 3 & 6 & 0 & -4.242477 & -4.540171 & -0.503601 \\
\hline 4 & 6 & 0 & -3.219373 & -5.116022 & -1.275053 \\
\hline 5 & 6 & 0 & -2.816639 & -4.573774 & -2.489294 \\
\hline 6 & 6 & 0 & -3.449158 & -3.401344 & -2.948516 \\
\hline 7 & 1 & 0 & -4.953249 & -1.955957 & -2.603354 \\
\hline 8 & 1 & 0 & -2.740872 & -6.024138 & -0.910386 \\
\hline 9 & 6 & 0 & -4.538628 & -5.171746 & 0.845168 \\
\hline 10 & 1 & 0 & -4.372536 & -6.255104 & 0.764307 \\
\hline 11 & 1 & 0 & -5.594040 & -5.047022 & 1.109783 \\
\hline 12 & 6 & 0 & -6.047466 & -2.720244 & -0.251044 \\
\hline 13 & 1 & 0 & -6.584082 & -3.447253 & 0.368664 \\
\hline 14 & 1 & 0 & -6.769889 & -2.381398 & -1.006934 \\
\hline 15 & 6 & 0 & -3.641759 & -4.635132 & 1.948183 \\
\hline 16 & 6 & 0 & -4.014299 & -3.591117 & 2.810931 \\
\hline 17 & 6 & 0 & -2.360736 & -5.186932 & 2.075807 \\
\hline 18 & 6 & 0 & -3.095113 & -3.158359 & 3.774340 \\
\hline 19 & 6 & 0 & -1.438371 & -4.716214 & 3.000940 \\
\hline 20 & 1 & 0 & -2.058507 & -6.008074 & 1.422914 \\
\hline 21 & 6 & 0 & -1.814457 & -3.686114 & 3.875040 \\
\hline 22 & 1 & 0 & -3.359853 & -2.360588 & 4.471031 \\
\hline 23 & 6 & 0 & -5.649198 & -1.514696 & 0.581628 \\
\hline 24 & 6 & 0 & -5.604027 & -0.265772 & -0.047064 \\
\hline 25 & 6 & 0 & -5.332361 & -1.591006 & 1.949926 \\
\hline 26 & 6 & 0 & -5.224330 & 0.894780 & 0.618276 \\
\hline 27 & 1 & 0 & -5.872978 & -0.170351 & -1.100917 \\
\hline 28 & 6 & 0 & -4.967354 & -0.416857 & 2.616399 \\
\hline 29 & 6 & 0 & -4.889179 & 0.815184 & 1.976889 \\
\hline 30 & 1 & 0 & -4.733783 & -0.442833 & 3.683014 \\
\hline 31 & 6 & 0 & -5.358165 & -2.885814 & 2.744552 \\
\hline 32 & 1 & 0 & -5.665643 & -2.644991 & 3.771692 \\
\hline 33 & 1 & 0 & -6.127362 & -3.559279 & 2.351232 \\
\hline 34 & 8 & 0 & -3.115863 & -2.850998 & -4.148543 \\
\hline 35 & 8 & 0 & -1.856014 & -5.090205 & -3.288439 \\
\hline 36 & 6 & 0 & -1.151444 & -6.231822 & -2.859192 \\
\hline 37 & 1 & 0 & -0.416463 & -6.460496 & -3.640068 \\
\hline 38 & 1 & 0 & -0.621835 & -6.048079 & -1.909543 \\
\hline 39 & 1 & 0 & -1.820777 & -7.098535 & -2.732529 \\
\hline 40 & 8 & 0 & -0.175480 & -5.234162 & 3.094766 \\
\hline 41 & 8 & 0 & -0.941386 & -3.154305 & 4.776251 \\
\hline 42 & 6 & 0 & -0.524176 & -4.003826 & 5.831649 \\
\hline 43 & 1 & 0 & -1.387182 & -4.335538 & 6.433434 \\
\hline 44 & 1 & 0 & 0.013995 & -4.883782 & 5.450822 \\
\hline 45 & 1 & 0 & 0.147811 & -3.415587 & 6.469757 \\
\hline 46 & 8 & 0 & -4.526122 & 1.932056 & 2.682951 \\
\hline 47 & 8 & 0 & -5.152798 & 2.066101 & -0.080872 \\
\hline
\end{tabular}




$\begin{array}{rrrrrr}48 & 6 & 0 & -6.072094 & 3.080762 & 0.296737 \\ 49 & 1 & 0 & -5.879270 & 3.943995 & -0.352449 \\ 50 & 1 & 0 & -7.110183 & 2.741288 & 0.144935 \\ 51 & 1 & 0 & -5.932323 & 3.373771 & 1.346947 \\ 52 & 6 & 0 & 0.669927 & -5.071269 & 1.966644 \\ 53 & 1 & 0 & 1.617585 & -5.564127 & 2.222932 \\ 54 & 1 & 0 & 0.255675 & -5.576540 & 1.077353 \\ 55 & 6 & 0 & 0.919171 & -3.603942 & 1.660042 \\ 56 & 1 & 0 & -0.010017 & -3.118048 & 1.324091 \\ 57 & 1 & 0 & 1.238420 & -3.085928 & 2.581083 \\ 58 & 6 & 0 & -1.786917 & -2.375954 & -4.320830 \\ 59 & 1 & 0 & -1.330881 & -2.887670 & -5.183256 \\ 60 & 1 & 0 & -1.175575 & -2.614395 & -3.438194 \\ 61 & 6 & 0 & -1.800706 & -0.884400 & -4.581427 \\ 62 & 1 & 0 & -0.772398 & -0.562385 & -4.836973 \\ 63 & 1 & 0 & -2.445398 & -0.675964 & -5.448082 \\ 64 & 6 & 0 & -3.129412 & 2.137277 & 2.858356 \\ 65 & 1 & 0 & -2.994793 & 2.645112 & 3.824372 \\ 66 & 1 & 0 & -2.603413 & 1.170149 & 2.902677 \\ 67 & 6 & 0 & -2.564007 & 2.966482 & 1.720139 \\ 68 & 1 & 0 & -2.902020 & 2.541018 & 0.767720 \\ 69 & 1 & 0 & -2.939724 & 4.005236 & 1.780024 \\ 70 & 8 & 0 & 1.909158 & -3.526515 & 0.659335 \\ 71 & 8 & 0 & -2.311527 & -0.121462 & -3.516885 \\ 72 & 8 & 0 & -1.153898 & 2.950457 & 1.809692 \\ 73 & 6 & 0 & 3.250664 & -0.949616 & 0.881956 \\ 74 & 6 & 0 & 1.949722 & -1.125173 & 0.094590 \\ 75 & 6 & 0 & 0.884786 & 0.632983 & 1.272842 \\ 76 & 6 & 0 & 2.106811 & 0.857508 & 2.172113 \\ 77 & 6 & 0 & 3.386651 & 0.466689 & 1.435886 \\ 78 & 1 & 0 & 2.002696 & -0.506735 & -0.817104 \\ 79 & 1 & 0 & 3.234364 & -1.645819 & 1.738592 \\ 80 & 1 & 0 & -0.046524 & 0.824154 & 1.816901 \\ 81 & 1 & 0 & 2.002488 & 0.182216 & 3.040032 \\ 82 & 1 & 0 & 3.534729 & 1.158734 & 0.591169 \\ 83 & 8 & 0 & 0.839005 & -0.708164 & 0.873948 \\ 84 & 8 & 0 & 0.993515 & 1.488630 & 0.163454 \\ 85 & 8 & 0 & 2.198755 & 2.190673 & 2.606907 \\ 86 & 8 & 0 & 4.501778 & 0.491414 & 2.298888 \\ 87 & 8 & 0 & 4.307859 & -1.281619 & 0.017850 \\ 88 & 6 & 0 & 1.539112 & 2.466264 & 3.833586 \\ 89 & 1 & 0 & 0.460126 & 2.264780 & 3.738357 \\ 90 & 6 & 0 & 5.230115 & 1.711378 & 2.362283 \\ 91 & 1 & 0 & 4.535615 & 2.561080 & 2.410366 \\ 92 & 1 & 0 & 5.783315 & 1.670913 & 3.312035 \\ 93 & 6 & 0 & 5.423334 & -1.963755 & 0.565746 \\ 94 & 1 & 0 & 5.114945 & -2.486198 & 1.486516 \\ 96 & 1 & 0 & 6.210750 & -1.245827 & 0.838591 \\ 97 & 6 & 0 & 6.199990 & 1.884276 & 1.216879 \\ & 0 & 0 & 7.412275 & 1.185178 & 1.205283\end{array}$




$\begin{array}{cccccc}98 & 6 & 0 & 5.903318 & 2.724866 & 0.140767 \\ 99 & 6 & 0 & 8.299892 & 1.313055 & 0.140763 \\ 100 & 1 & 0 & 7.665749 & 0.537678 & 2.048814 \\ 101 & 6 & 0 & 6.788112 & 2.855360 & -0.929610 \\ 102 & 1 & 0 & 4.970356 & 3.294465 & 0.142899 \\ 103 & 6 & 0 & 7.986705 & 2.147006 & -0.933109 \\ 104 & 1 & 0 & 9.244586 & 0.764601 & 0.150064 \\ 105 & 1 & 0 & 6.539868 & 3.516451 & -1.762806 \\ 106 & 1 & 0 & 8.682500 & 2.249511 & -1.768717 \\ 107 & 6 & 0 & 5.941644 & -2.959874 & -0.438154 \\ 108 & 6 & 0 & 5.210966 & -4.122540 & -0.711173 \\ 109 & 6 & 0 & 7.133157 & -2.732632 & -1.128054 \\ 110 & 6 & 0 & 5.667500 & -5.038805 & -1.653450 \\ 111 & 1 & 0 & 4.273521 & -4.302327 & -0.177190 \\ 112 & 6 & 0 & 7.594466 & -3.650623 & -2.072402 \\ 113 & 1 & 0 & 7.707367 & -1.825265 & -0.923402 \\ 114 & 6 & 0 & 6.862024 & -4.804690 & -2.336558 \\ 115 & 1 & 0 & 5.091267 & -5.944408 & -1.856486 \\ 116 & 1 & 0 & 8.529909 & -3.461433 & -2.603688 \\ 117 & 1 & 0 & 7.221255 & -5.525259 & -3.074694 \\ 118 & 6 & 0 & 1.723172 & -2.560639 & -0.347137 \\ 119 & 1 & 0 & 2.452218 & -2.794656 & -1.133156 \\ 120 & 1 & 0 & 0.708018 & -2.634684 & -0.777895 \\ 121 & 6 & 0 & -0.148090 & 2.161404 & -0.289356 \\ 122 & 8 & 0 & -1.242559 & 1.274061 & -0.362696 \\ 123 & 6 & 0 & -1.835747 & 1.244630 & -1.655834 \\ 124 & 6 & 0 & -1.285358 & 2.475631 & -2.380598 \\ 125 & 6 & 0 & 0.096630 & 2.648372 & -1.744264 \\ 126 & 1 & 0 & -2.927089 & 1.312696 & -1.532399 \\ 127 & 1 & 0 & -1.191965 & 2.301399 & -3.464771 \\ 128 & 1 & 0 & 0.402491 & 3.707512 & -1.767803 \\ 129 & 6 & 0 & -0.493732 & 3.344469 & 0.630878 \\ 130 & 1 & 0 & 0.450032 & 3.829548 & 0.917963 \\ 131 & 1 & 0 & -1.108037 & 4.064487 & 0.068321 \\ 132 & 8 & 0 & 1.002084 & 1.850904 & -2.453054 \\ 133 & 8 & 0 & -2.031709 & 3.641255 & -2.127960 \\ 134 & 6 & 0 & -1.513917 & -0.064505 & -2.359296 \\ 135 & 1 & 0 & -1.732388 & -0.896922 & -1.667114 \\ 136 & 1 & 0 & -0.441041 & -0.097427 & -2.613629 \\ 137 & 6 & 0 & -3.275345 & 3.716072 & -2.786349 \\ 138 & 1 & 0 & -4.016127 & 3.049981 & -2.310268 \\ 139 & 1 & 0 & -3.158101 & 3.373648 & -3.833143 \\ 140 & 1 & 0 & 1.946308 & 1.808835 & 4.622756 \\ 141 & 6 & 0 & 1.758331 & 3.909754 & 4.195920 \\ 142 & 6 & 0 & 2.933798 & 4.309212 & 4.838659 \\ 143 & 6 & 0 & 0.805612 & 4.876877 & 3.864203 \\ 144 & 6 & 0 & 3.154089 & 5.649409 & 5.146165 \\ 146 & 1 & 0 & 3.683014 & 3.558972 & 5.104721 \\ 147 & 1 & 0 & -0.109979 & 4.562897 & 3.356811\end{array}$




$\begin{array}{rrrrrr}148 & 6 & 0 & 2.197242 & 6.607421 & 4.813238 \\ 149 & 1 & 0 & 4.075056 & 5.948346 & 5.651692 \\ 150 & 1 & 0 & 0.269431 & 6.965603 & 3.911165 \\ 151 & 1 & 0 & 2.367213 & 7.658622 & 5.056678 \\ 152 & 6 & 0 & 3.136794 & 1.451753 & -3.419246 \\ 153 & 6 & 0 & 2.931241 & 1.660931 & -4.787536 \\ 154 & 6 & 0 & 4.045610 & 0.471494 & -3.014600 \\ 155 & 6 & 0 & 3.622616 & 0.909572 & -5.732527 \\ 156 & 1 & 0 & 2.220559 & 2.425023 & -5.112851 \\ 157 & 6 & 0 & 4.743098 & -0.280475 & -3.960785 \\ 158 & 1 & 0 & 4.209215 & 0.275163 & -1.952419 \\ 159 & 6 & 0 & 4.533658 & -0.063557 & -5.319732 \\ 160 & 1 & 0 & 3.454662 & 1.085276 & -6.797475 \\ 161 & 1 & 0 & 5.450820 & -1.043416 & -3.628092 \\ 162 & 1 & 0 & 5.079441 & -0.652227 & -6.060497 \\ 163 & 6 & 0 & 2.360259 & 2.258687 & -2.413953 \\ 164 & 1 & 0 & 2.773291 & 2.122197 & -1.404033 \\ 165 & 1 & 0 & 2.419131 & 3.334450 & -2.662382 \\ 166 & 6 & 0 & -3.790487 & 5.133226 & -2.773274 \\ 167 & 6 & 0 & -2.923433 & 6.220971 & -2.653635 \\ 168 & 6 & 0 & -5.159864 & 5.373412 & -2.922798 \\ 169 & 6 & 0 & -3.416663 & 7.524334 & -2.682250 \\ 170 & 1 & 0 & -1.855450 & 6.037224 & -2.531843 \\ 171 & 6 & 0 & -5.652888 & 6.675357 & -2.958813 \\ 172 & 1 & 0 & -5.849768 & 4.530003 & -3.013331 \\ 173 & 6 & 0 & -4.781473 & 7.756544 & -2.836871 \\ 174 & 1 & 0 & -2.727042 & 8.365885 & -2.583815 \\ 175 & 1 & 0 & -6.725247 & 6.846258 & -3.076701 \\ 176 & 1 & 0 & -5.166939 & 8.778160 & -2.859367 \\ -------------------------------------------------------\end{array}$

Compound $\boldsymbol{P} \mathbf{- 8}$, conformer 3

\begin{tabular}{cccccc} 
Center & \multicolumn{2}{c}{ Atomic } & \multicolumn{2}{c}{ Atomic } & \multicolumn{3}{c}{ Coordinates } & (Angstroms) \\
Number & Number & Type & X & Y & Z \\
-1 & 6 & 0 & 4.986901 & -0.726446 & 2.492356 \\
2 & 6 & 0 & 4.862443 & -2.123736 & 2.462103 \\
3 & 6 & 0 & 3.988988 & -2.731129 & 3.372698 \\
4 & 6 & 0 & 3.298526 & -1.930941 & 4.298207 \\
5 & 6 & 0 & 3.435143 & -0.548258 & 4.325677 \\
6 & 6 & 0 & 4.286354 & 0.065627 & 3.385203 \\
7 & 1 & 0 & 5.649467 & -0.212659 & 1.793611 \\
8 & 1 & 0 & 2.648635 & -2.417259 & 5.024659 \\
9 & 6 & 0 & 3.707759 & -4.223422 & 3.389757 \\
10 & 1 & 0 & 3.517044 & -4.526608 & 4.428870 \\
11 & 1 & 0 & 4.589971 & -4.787183 & 3.068295 \\
12 & 6 & 0 & 5.675765 & -2.893634 & 1.434703 \\
13 & 1 & 0 & 5.886728 & -3.905414 & 1.798265 \\
14 & 1 & 0 & 6.653041 & -2.399464 & 1.341099
\end{tabular}




$\begin{array}{cccccc}15 & 6 & 0 & 2.495785 & -4.604458 & 2.556225 \\ 16 & 6 & 0 & 2.576467 & -5.059119 & 1.229814 \\ 17 & 6 & 0 & 1.231335 & -4.450679 & 3.137359 \\ 18 & 6 & 0 & 1.388173 & -5.362694 & 0.554057 \\ 19 & 6 & 0 & 0.056496 & -4.696167 & 2.438729 \\ 20 & 1 & 0 & 1.153577 & -4.106752 & 4.170159 \\ 21 & 6 & 0 & 0.134399 & -5.176300 & 1.122717 \\ 22 & 1 & 0 & 1.416479 & -5.736651 & -0.471331 \\ 23 & 6 & 0 & 5.050410 & -2.950780 & 0.052208 \\ 24 & 6 & 0 & 5.302198 & -1.891479 & -0.825957 \\ 25 & 6 & 0 & 4.233712 & -4.008524 & -0.386171 \\ 26 & 6 & 0 & 4.748822 & -1.825740 & -2.099637 \\ 27 & 1 & 0 & 5.951949 & -1.068076 & -0.523109 \\ 28 & 6 & 0 & 3.699278 & -3.946656 & -1.677236 \\ 29 & 6 & 0 & 3.924048 & -2.873453 & -2.531988 \\ 30 & 1 & 0 & 3.077003 & -4.762571 & -2.051236 \\ 31 & 6 & 0 & 3.885435 & -5.212011 & 0.474057 \\ 32 & 1 & 0 & 3.797399 & -6.085971 & -0.186293 \\ 33 & 1 & 0 & 4.705040 & -5.438279 & 1.164797 \\ 34 & 8 & 0 & 4.490322 & 1.411960 & 3.396760 \\ 35 & 8 & 0 & 2.816686 & 0.273876 & 5.203352 \\ 36 & 6 & 0 & 1.900612 & -0.272970 & 6.123059 \\ 37 & 1 & 0 & 1.506351 & 0.564656 & 6.710606 \\ 38 & 1 & 0 & 1.064721 & -0.777534 & 5.610363 \\ 39 & 1 & 0 & 2.387224 & -0.990052 & 6.804374 \\ 40 & 8 & 0 & -1.177348 & -4.487754 & 2.990500 \\ 41 & 8 & 0 & -0.978513 & -5.404288 & 0.370104 \\ 42 & 6 & 0 & -1.846897 & -6.434869 & 0.810186 \\ 43 & 1 & 0 & -2.669166 & -6.488752 & 0.085297 \\ 44 & 1 & 0 & -1.324505 & -7.406364 & 0.830982 \\ 45 & 1 & 0 & -2.255018 & -6.219199 & 1.807770 \\ 46 & 8 & 0 & 3.370635 & -2.868457 & -3.785640 \\ 47 & 8 & 0 & 4.989895 & -0.722350 & -2.867980 \\ 48 & 6 & 0 & 5.743592 & -0.929216 & -4.052816 \\ 49 & 1 & 0 & 5.847957 & 0.048784 & -4.539490 \\ 50 & 1 & 0 & 6.746663 & -1.319849 & -3.813704 \\ 51 & 1 & 0 & 5.232949 & -1.624866 & -4.734008 \\ 52 & 6 & 0 & -1.457260 & -3.181045 & 3.469489 \\ 53 & 1 & 0 & -2.480400 & -3.216866 & 3.867425 \\ 54 & 1 & 0 & -0.784119 & -2.899198 & 4.296484 \\ 55 & 6 & 0 & -1.369795 & -2.158453 & 2.350299 \\ 56 & 1 & 0 & -0.333168 & -2.079040 & 1.985453 \\ 57 & 1 & 0 & -1.983790 & -2.498712 & 1.498656 \\ 58 & 6 & 0 & 3.375683 & 2.263856 & 3.166489 \\ 59 & 1 & 0 & 3.283051 & 2.965716 & 4.010461 \\ 60 & 1 & 0 & 2.445517 & 1.679048 & 3.124734 \\ 64 & 6 & 0 & 3.575678 & 3.052994 & 1.889690 \\ & 1 & 0 & 2.762874 & 3.801003 & 1.809512 \\ & 1 & 0 & 4.530358 & 3.596522 & 1.944802 \\ 63 & 0 & 2.018874 & -2.433942 & -3.873333\end{array}$




$\begin{array}{cccccc}65 & 1 & 0 & 1.554035 & -2.981770 & -4.705841 \\ 66 & 1 & 0 & 1.474201 & -2.686516 & -2.949255 \\ 67 & 6 & 0 & 1.958380 & -0.935341 & -4.101889 \\ 68 & 1 & 0 & 2.613359 & -0.439693 & -3.375088 \\ 69 & 1 & 0 & 2.330730 & -0.689933 & -5.114697 \\ 70 & 8 & 0 & -1.811725 & -0.916878 & 2.847687 \\ 71 & 8 & 0 & 3.639137 & 2.261643 & 0.728870 \\ 72 & 8 & 0 & 0.621439 & -0.507030 & -3.954987 \\ 73 & 6 & 0 & -3.122439 & 0.457045 & 0.630718 \\ 74 & 6 & 0 & -1.608647 & 0.515960 & 0.847353 \\ 75 & 6 & 0 & -1.171385 & -0.238085 & -1.363212 \\ 76 & 6 & 0 & -2.662232 & -0.453321 & -1.651356 \\ 77 & 6 & 0 & -3.484428 & 0.550164 & -0.848930 \\ 78 & 1 & 0 & -1.258816 & 1.532282 & 0.601389 \\ 79 & 1 & 0 & -3.492401 & -0.513684 & 1.005553 \\ 80 & 1 & 0 & -0.547957 & -0.983575 & -1.869961 \\ 81 & 1 & 0 & -2.914295 & -1.465091 & -1.289780 \\ 82 & 1 & 0 & -3.248307 & 1.565316 & -1.209241 \\ 83 & 8 & 0 & -0.945992 & -0.417800 & 0.008223 \\ 84 & 8 & 0 & -0.837639 & 1.058162 & -1.778003 \\ 85 & 8 & 0 & -2.992878 & -0.323447 & -3.009521 \\ 86 & 8 & 0 & -4.866156 & 0.297325 & -0.971861 \\ 87 & 8 & 0 & -3.686778 & 1.502646 & 1.381920 \\ 88 & 6 & 0 & -2.631893 & -1.408030 & -3.852898 \\ 89 & 1 & 0 & -2.982836 & -1.111343 & -4.852396 \\ 90 & 6 & 0 & -5.548209 & 1.080361 & -1.921468 \\ 91 & 1 & 0 & -5.500377 & 2.150003 & -1.641200 \\ 92 & 1 & 0 & -5.063763 & 0.982101 & -2.907849 \\ 93 & 6 & 0 & -5.003904 & 1.329268 & 1.871770 \\ 94 & 1 & 0 & -5.259995 & 0.256779 & 1.868161 \\ 95 & 1 & 0 & -5.729638 & 1.836442 & 1.215249 \\ 96 & 6 & 0 & -6.989471 & 0.650696 & -2.019413 \\ 97 & 6 & 0 & -7.421514 & -0.577197 & -1.515044 \\ 98 & 6 & 0 & -7.916638 & 1.483924 & -2.654410 \\ 99 & 6 & 0 & -8.754816 & -0.966025 & -1.645807 \\ 100 & 1 & 0 & -6.702128 & -1.224701 & -1.013708 \\ 101 & 6 & 0 & -9.245815 & 1.094735 & -2.789413 \\ 102 & 1 & 0 & -7.592812 & 2.451981 & -3.046730 \\ 103 & 6 & 0 & -9.670647 & -0.134110 & -2.284071 \\ 104 & 1 & 0 & -9.078891 & -1.928603 & -1.243148 \\ 105 & 1 & 0 & -9.957180 & 1.757383 & -3.287565 \\ 106 & 1 & 0 & -10.714498 & -0.438966 & -2.385717 \\ 107 & 6 & 0 & -5.097784 & 1.873462 & 3.274430 \\ 108 & 6 & 0 & -4.346528 & 1.286499 & 4.300354 \\ 109 & 6 & 0 & -5.917087 & 2.961500 & 3.575309 \\ 110 & 6 & 0 & -4.418273 & 1.780197 & 5.598770 \\ 111 & 1 & 0 & -3.699753 & 0.434834 & 4.071478 \\ 114 & 6 & 0 & -5.993427 & 3.456982 & 4.877930 \\ & 1 & 0 & -6.504558 & 3.428415 & 2.780419 \\ & & 0 & -5.243434 & 2.867805 & 5.891298\end{array}$




$\begin{array}{llllll}115 & 1 & 0 & -3.829716 & 1.313287 & 6.391902 \\ 116 & 1 & 0 & -6.640158 & 4.309104 & 5.098713 \\ 117 & 1 & 0 & -5.300125 & 3.253943 & 6.911478 \\ 118 & 6 & 0 & -1.216995 & 0.229922 & 2.287047 \\ 119 & 1 & 0 & -1.542761 & 1.075989 & 2.905180 \\ 120 & 1 & 0 & -0.114984 & 0.159816 & 2.339418 \\ 121 & 6 & 0 & 0.430332 & 1.302507 & -2.314947 \\ 122 & 8 & 0 & 1.420779 & 0.609614 & -1.590143 \\ 123 & 6 & 0 & 2.480257 & 1.459551 & -1.164707 \\ 124 & 6 & 0 & 2.289045 & 2.772516 & -1.929264 \\ 125 & 6 & 0 & 0.771005 & 2.803717 & -2.162963 \\ 126 & 1 & 0 & 3.433264 & 0.980301 & -1.435355 \\ 127 & 1 & 0 & 2.616208 & 3.634386 & -1.326952 \\ 128 & 1 & 0 & 0.534463 & 3.364093 & -3.083520 \\ 129 & 6 & 0 & 0.476720 & 0.882363 & -3.795325 \\ 130 & 1 & 0 & -0.474539 & 1.185923 & -4.256453 \\ 131 & 1 & 0 & 1.298546 & 1.417726 & -4.294978 \\ 132 & 8 & 0 & 0.096529 & 3.341038 & -1.060878 \\ 133 & 8 & 0 & 2.924227 & 2.774424 & -3.187672 \\ 134 & 6 & 0 & 2.441195 & 1.630665 & 0.345793 \\ 135 & 1 & 0 & 2.335683 & 0.634331 & 0.810399 \\ 136 & 1 & 0 & 1.563653 & 2.237549 & 0.627414 \\ 137 & 6 & 0 & 4.332887 & 2.919746 & -3.167963 \\ 138 & 1 & 0 & 4.643746 & 2.802638 & -4.216604 \\ 139 & 1 & 0 & 4.806554 & 2.103961 & -2.596504 \\ 140 & 1 & 0 & -1.536356 & -1.510954 & -3.909079 \\ 141 & 6 & 0 & -3.261427 & -2.723771 & -3.457741 \\ 142 & 6 & 0 & -4.651933 & -2.839098 & -3.348943 \\ 143 & 6 & 0 & -2.470527 & -3.840974 & -3.185579 \\ 144 & 6 & 0 & -5.235939 & -4.046368 & -2.979386 \\ 145 & 1 & 0 & -5.280830 & -1.968541 & -3.550089 \\ 146 & 6 & 0 & -3.052062 & -5.054641 & -2.817875 \\ 147 & 1 & 0 & -1.382705 & -3.759601 & -3.254970 \\ 148 & 6 & 0 & -4.436263 & -5.159441 & -2.714142 \\ 149 & 1 & 0 & -6.322704 & -4.122004 & -2.898370 \\ 150 & 1 & 0 & -2.417087 & -5.916806 & -2.603100 \\ 151 & 1 & 0 & -4.895027 & -6.107408 & -2.424347 \\ 152 & 6 & 0 & -0.751788 & 5.210812 & 0.160569 \\ 153 & 6 & 0 & -0.346794 & 6.367119 & 0.831151 \\ 154 & 6 & 0 & -1.860591 & 4.506604 & 0.640173 \\ 155 & 6 & 0 & -1.040521 & 6.818433 & 1.953242 \\ 156 & 1 & 0 & 0.524798 & 6.921624 & 0.473689 \\ 157 & 6 & 0 & -2.545913 & 4.946859 & 1.768999 \\ 158 & 1 & 0 & -2.188455 & 3.598326 & 0.134596 \\ 159 & 6 & 0 & -2.140458 & 6.107464 & 2.427191 \\ 160 & 1 & 0 & -0.711265 & 7.725237 & 2.465491 \\ 161 & 1 & 0 & -3.397897 & 4.369860 & 2.135082 \\ 162 & 1 & 0 & -2.678837 & 6.453865 & 3.312177 \\ 163 & 6 & 0 & -0.018090 & 4.747733 & -1.070668 \\ 164 & 1 & 0 & -0.563909 & 5.061033 & -1.982305\end{array}$




\begin{tabular}{|c|c|c|c|c|c|}
\hline 165 & 1 & 0 & 0.975530 & 5.228543 & -1.113646 \\
\hline 166 & 6 & 0 & 4.788689 & 4.259771 & -2.638518 \\
\hline 167 & 6 & 0 & 4.296885 & 5.441542 & -3.203573 \\
\hline 168 & 6 & 0 & 5.700120 & 4.343062 & -1.585130 \\
\hline 169 & 6 & 0 & 4.714947 & 6.680677 & -2.728442 \\
\hline 170 & 1 & 0 & 3.576141 & 5.384127 & -4.022920 \\
\hline 171 & 6 & 0 & 6.126606 & 5.583630 & -1.110855 \\
\hline 172 & 1 & 0 & 6.071304 & 3.425380 & -1.122567 \\
\hline 173 & 6 & 0 & 5.634679 & 6.754358 & -1.681433 \\
\hline 174 & 1 & 0 & 4.325259 & 7.596348 & -3.178887 \\
\hline 175 & 1 & 0 & 6.841737 & 5.633887 & -0.286791 \\
\hline 176 & 1 & 0 & 5.964766 & 7.726840 & -1.309598 \\
\hline
\end{tabular}

Compound $\boldsymbol{P} \mathbf{- 8}$, conformer 4

\begin{tabular}{|c|c|c|c|c|c|}
\hline \multirow{2}{*}{$\begin{array}{l}\text { Center } \\
\text { Number }\end{array}$} & Atomic & \multirow{2}{*}{\multicolumn{2}{|c|}{$\begin{array}{r}\text { Atomic } \\
\text { Type }\end{array}$}} & \multicolumn{2}{|c|}{ Coordinates (Angstroms) } \\
\hline & Numbe & & & $X$ & $\mathrm{Z}$ \\
\hline 1 & 8 & 0 & 2.828283 & 0.592454 & -5.032082 \\
\hline 2 & 6 & 0 & -1.233508 & 4.982686 & -0.535253 \\
\hline 3 & 1 & 0 & -2.251598 & 5.387012 & -0.454389 \\
\hline 4 & 1 & 0 & -0.881344 & 5.159059 & -1.565714 \\
\hline 5 & 6 & 0 & -1.244145 & 3.494497 & -0.232241 \\
\hline 6 & 1 & 0 & -0.244083 & 3.061894 & -0.395951 \\
\hline 7 & 1 & 0 & -1.489127 & 3.342598 & 0.833271 \\
\hline 8 & 6 & 0 & 1.554521 & -0.032073 & -4.935885 \\
\hline 9 & 1 & 0 & 1.064728 & -0.008101 & -5.922297 \\
\hline 10 & 1 & 0 & 0.908036 & 0.518049 & -4.236993 \\
\hline 11 & 6 & 0 & 1.718498 & -1.472858 & -4.499932 \\
\hline 12 & 1 & 0 & 0.731189 & -1.971304 & -4.557955 \\
\hline 13 & 1 & 0 & 2.397711 & -1.985778 & -5.196822 \\
\hline 14 & 6 & 0 & 3.386207 & -0.502414 & 3.465341 \\
\hline 15 & 1 & 0 & 3.317082 & -0.563437 & 4.560926 \\
\hline 16 & 1 & 0 & 2.759608 & 0.339412 & 3.128271 \\
\hline 17 & 6 & 0 & 2.900999 & -1.784231 & 2.814527 \\
\hline 18 & 1 & 0 & 3.185320 & -1.778614 & 1.755053 \\
\hline 19 & 1 & 0 & 3.384992 & -2.658963 & 3.288890 \\
\hline 20 & 8 & 0 & -2.190454 & 2.879549 & -1.075455 \\
\hline 21 & 8 & 0 & 2.278488 & -1.624341 & -3.219528 \\
\hline 22 & 8 & 0 & 1.499675 & -1.859197 & 2.962978 \\
\hline 23 & 6 & 0 & -3.306554 & 0.648107 & 0.429894 \\
\hline 24 & 6 & 0 & -2.013972 & 0.517961 & -0.379640 \\
\hline 25 & 6 & 0 & -0.814353 & -0.311567 & 1.492111 \\
\hline 26 & 6 & 0 & -2.022908 & -0.131304 & 2.419990 \\
\hline 27 & 6 & 0 & -3.319812 & -0.295537 & 1.629806 \\
\hline 28 & 1 & 0 & -2.000143 & -0.473326 & -0.863477 \\
\hline 29 & 1 & 0 & -3.373094 & 1.680016 & 0.815889 \\
\hline 30 & 1 & 0 & 0.125662 & -0.118791 & 2.020908 \\
\hline 31 & 1 & 0 & -1.986455 & 0.908583 & 2.787462 \\
\hline
\end{tabular}




$\begin{array}{rrrrrr}32 & 1 & 0 & -3.382944 & -1.331885 & 1.260678 \\ 33 & 8 & 0 & -0.880677 & 0.638077 & 0.465460 \\ 34 & 8 & 0 & -0.832242 & -1.620770 & 0.981548 \\ 35 & 8 & 0 & -2.023038 & -1.028601 & 3.500538 \\ 36 & 8 & 0 & -4.449256 & 0.020608 & 2.412776 \\ 37 & 8 & 0 & -4.374077 & 0.400610 & -0.449937 \\ 38 & 6 & 0 & -1.163582 & -0.708816 & 4.585791 \\ 39 & 1 & 0 & -1.312577 & -1.525566 & 5.307620 \\ 40 & 6 & 0 & -5.057036 & -1.056252 & 3.114551 \\ 41 & 1 & 0 & -4.286912 & -1.661436 & 3.612030 \\ 42 & 1 & 0 & -5.674496 & -0.582888 & 3.891777 \\ 43 & 6 & 0 & -5.557162 & 1.164891 & -0.288302 \\ 44 & 1 & 0 & -5.318147 & 2.098239 & 0.247647 \\ 45 & 1 & 0 & -6.286156 & 0.609935 & 0.320268 \\ 46 & 6 & 0 & -5.924726 & -1.927748 & 2.236783 \\ 47 & 6 & 0 & -7.193829 & -1.492337 & 1.838780 \\ 48 & 6 & 0 & -5.475451 & -3.173309 & 1.790281 \\ 49 & 6 & 0 & -7.988032 & -2.275144 & 1.005773 \\ 50 & 1 & 0 & -7.567036 & -0.528981 & 2.196186 \\ 51 & 6 & 0 & -6.266736 & -3.961618 & 0.954843 \\ 52 & 1 & 0 & -4.494901 & -3.537558 & 2.107887 \\ 53 & 6 & 0 & -7.523119 & -3.512025 & 0.557755 \\ 54 & 1 & 0 & -8.978467 & -1.922868 & 0.708594 \\ 55 & 1 & 0 & -5.899422 & -4.932440 & 0.615005 \\ 56 & 1 & 0 & -8.145600 & -4.127974 & -0.094918 \\ 57 & 6 & 0 & -6.134974 & 1.486124 & -1.641883 \\ 58 & 6 & 0 & -5.507865 & 2.429660 & -2.464466 \\ 59 & 6 & 0 & -7.278275 & 0.836311 & -2.108938 \\ 60 & 6 & 0 & -6.018128 & 2.716995 & -3.726629 \\ 61 & 1 & 0 & -4.608109 & 2.937724 & -2.105911 \\ 62 & 6 & 0 & -7.793373 & 1.123532 & -3.373623 \\ 63 & 1 & 0 & -7.771985 & 0.095655 & -1.474427 \\ 64 & 6 & 0 & -7.163683 & 2.063999 & -4.184292 \\ 65 & 1 & 0 & -5.522622 & 3.457320 & -4.358909 \\ 66 & 1 & 0 & -8.690224 & 0.608996 & -3.725802 \\ 67 & 1 & 0 & -7.565248 & 2.291280 & -5.174343 \\ 68 & 6 & 0 & -1.908305 & 1.560926 & -1.479423 \\ 69 & 1 & 0 & -2.643305 & 1.316045 & -2.256431 \\ 70 & 1 & 0 & -0.896521 & 1.497236 & -1.920281 \\ 71 & 6 & 0 & 0.383886 & -2.300061 & 0.822654 \\ 72 & 8 & 0 & 1.355417 & -1.444470 & 0.263778 \\ 73 & 6 & 0 & 1.942732 & -1.980490 & -0.915520 \\ 74 & 6 & 0 & 1.546031 & -3.459113 & -0.928842 \\ 75 & 6 & 0 & 0.196154 & -3.449144 & -0.205242 \\ 76 & 1 & 0 & 3.034154 & -1.863094 & -0.837211 \\ 81 & 1 & 0 & 1.430517 & -3.839796 & -1.956710 \\ & 1 & 0 & 0.020619 & -4.414664 & 0.297855 \\ 77 & 6 & 0 & 0.884893 & -2.845110 & 2.170992 \\ 79 & 0 & 0.012498 & -3.231460 & 2.718194 \\ 75 & & & & & \\ 75 & 1 & 01315 & -3.676599 & 1.981832\end{array}$




$\begin{array}{llllll}82 & 8 & 0 & -0.800194 & -3.191662 & -1.153275 \\ 83 & 8 & 0 & 2.428283 & -4.272682 & -0.194414 \\ 84 & 6 & 0 & 1.471836 & -1.215471 & -2.142217 \\ 85 & 1 & 0 & 1.571506 & -0.134587 & -1.938284 \\ 86 & 1 & 0 & 0.407873 & -1.434578 & -2.336159 \\ 87 & 6 & 0 & 3.685325 & -4.495866 & -0.791765 \\ 88 & 1 & 0 & 4.338096 & -3.612139 & -0.681876 \\ 89 & 1 & 0 & 3.551339 & -4.666074 & -1.877855 \\ 90 & 1 & 0 & -0.109030 & -0.742684 & 4.270137 \\ 91 & 6 & 0 & -1.472861 & 0.617079 & 5.241543 \\ 92 & 6 & 0 & -0.490257 & 1.601671 & 5.357060 \\ 93 & 6 & 0 & -2.752793 & 0.879249 & 5.743176 \\ 94 & 6 & 0 & -0.772547 & 2.822948 & 5.969468 \\ 95 & 1 & 0 & 0.509574 & 1.413240 & 4.957532 \\ 96 & 6 & 0 & -3.039752 & 2.097379 & 6.350584 \\ 97 & 1 & 0 & -3.530454 & 0.117157 & 5.652794 \\ 98 & 6 & 0 & -2.048327 & 3.073078 & 6.467058 \\ 99 & 1 & 0 & 0.007999 & 3.582642 & 6.049901 \\ 100 & 1 & 0 & -4.042287 & 2.288865 & 6.739826 \\ 101 & 1 & 0 & -2.273603 & 4.028864 & 6.945349 \\ 102 & 6 & 0 & -2.956478 & -3.575732 & -2.077717 \\ 103 & 6 & 0 & -3.958772 & -2.610059 & -2.194644 \\ 104 & 6 & 0 & -2.732058 & -4.451403 & -3.145821 \\ 105 & 6 & 0 & -4.728317 & -2.526422 & -3.355622 \\ 106 & 1 & 0 & -4.140923 & -1.902561 & -1.382227 \\ 107 & 6 & 0 & -3.496023 & -4.367634 & -4.305525 \\ 108 & 1 & 0 & -1.948557 & -5.209114 & -3.063336 \\ 109 & 6 & 0 & -4.499384 & -3.403724 & -4.411920 \\ 110 & 1 & 0 & -5.508518 & -1.765383 & -3.431738 \\ 111 & 1 & 0 & -3.312227 & -5.059622 & -5.130489 \\ 112 & 1 & 0 & -5.101950 & -3.338494 & -5.320667 \\ 113 & 6 & 0 & -2.100852 & -3.666436 & -0.843022 \\ 114 & 1 & 0 & -2.538358 & -3.078986 & -0.022880 \\ 115 & 1 & 0 & -2.028531 & -4.717118 & -0.506922 \\ 116 & 6 & 0 & 4.354616 & -5.696069 & -0.170759 \\ 117 & 6 & 0 & 3.611273 & -6.707948 & 0.439701 \\ 118 & 6 & 0 & 5.745388 & -5.824778 & -0.236818 \\ 119 & 6 & 0 & 4.246848 & -7.827268 & 0.974482 \\ 120 & 1 & 0 & 2.526939 & -6.607894 & 0.497818 \\ 121 & 6 & 0 & 6.380877 & -6.945999 & 0.290867 \\ 122 & 1 & 0 & 6.339199 & -5.037291 & -0.708758 \\ 123 & 6 & 0 & 5.632378 & -7.951551 & 0.900624 \\ 124 & 1 & 0 & 3.652913 & -8.609691 & 1.452541 \\ 125 & 1 & 0 & 7.468133 & -7.031726 & 0.230957 \\ 126 & 1 & 0 & 6.129437 & -8.828956 & 1.320010 \\ 127 & 6 & 0 & 1.737385 & 5.454359 & -0.572881 \\ 130 & 6 & 0 & 3.078984 & 5.060897 & -0.493180 \\ 131 & 6 & 0 & 3.570471 & 4.589303 & 0.735009 \\ & 6 & 0 & 2.701853 & 4.549873 & 1.832523 \\ 19 & 0 & 1.362562 & 4.906457 & 1.739982\end{array}$




\begin{tabular}{|c|c|c|c|c|c|}
\hline 132 & 6 & 0 & 0.869445 & 5.359001 & 0.507014 \\
\hline 133 & 1 & 0 & 1.343245 & 5.833862 & -1.517384 \\
\hline 134 & 1 & 0 & 3.057008 & 4.203944 & 2.805252 \\
\hline 135 & 6 & 0 & 4.991805 & 4.091657 & 0.934049 \\
\hline 136 & 1 & 0 & 5.319804 & 4.392609 & 1.938781 \\
\hline 137 & 1 & 0 & 5.674327 & 4.586832 & 0.234832 \\
\hline 138 & 6 & 0 & 3.910961 & 5.120303 & -1.763397 \\
\hline 139 & 1 & 0 & 4.971612 & 5.254053 & -1.525530 \\
\hline 140 & 1 & 0 & 3.611776 & 6.014470 & -2.328095 \\
\hline 141 & 6 & 0 & 5.131936 & 2.582072 & 0.830095 \\
\hline 142 & 6 & 0 & 5.468616 & 1.914450 & -0.361270 \\
\hline 143 & 6 & 0 & 4.906516 & 1.819669 & 1.980623 \\
\hline 144 & 6 & 0 & 5.582859 & 0.520365 & -0.338337 \\
\hline 145 & 6 & 0 & 4.984922 & 0.431639 & 1.987330 \\
\hline 146 & 1 & 0 & 4.659864 & 2.311978 & 2.923850 \\
\hline 147 & 6 & 0 & 5.341427 & -0.232405 & 0.805587 \\
\hline 148 & 1 & 0 & 5.870330 & -0.023024 & -1.240625 \\
\hline 149 & 6 & 0 & 3.706181 & 3.904119 & -2.649567 \\
\hline 150 & 6 & 0 & 2.626439 & 3.933910 & -3.547635 \\
\hline 151 & 6 & 0 & 4.503991 & 2.755357 & -2.577997 \\
\hline 152 & 6 & 0 & 2.304152 & 2.847577 & -4.351785 \\
\hline 153 & 1 & 0 & 2.036143 & 4.846352 & -3.621694 \\
\hline 154 & 6 & 0 & 4.161133 & 1.658293 & -3.382950 \\
\hline 155 & 6 & 0 & 3.078183 & 1.674718 & -4.245076 \\
\hline 156 & 1 & 0 & 4.747927 & 0.738484 & -3.347078 \\
\hline 157 & 6 & 0 & 5.721305 & 2.630084 & -1.676191 \\
\hline 158 & 1 & 0 & 6.486678 & 2.063176 & -2.224770 \\
\hline 159 & 1 & 0 & 6.157877 & 3.617954 & -1.491546 \\
\hline 160 & 8 & 0 & -0.452624 & 5.696187 & 0.411077 \\
\hline 161 & 8 & 0 & 0.554609 & 4.749053 & 2.826093 \\
\hline 162 & 6 & 0 & -0.015037 & 5.925783 & 3.374410 \\
\hline 163 & 1 & 0 & -0.613075 & 5.615566 & 4.240818 \\
\hline 164 & 1 & 0 & 0.771220 & 6.621383 & 3.713590 \\
\hline 165 & 1 & 0 & -0.663837 & 6.435388 & 2.647934 \\
\hline 166 & 8 & 0 & 4.750957 & -0.263013 & 3.145230 \\
\hline 167 & 8 & 0 & 5.418947 & -1.593993 & 0.731781 \\
\hline 168 & 6 & 0 & 6.446977 & -2.208176 & 1.495023 \\
\hline 169 & 1 & 0 & 6.365358 & -3.289581 & 1.328674 \\
\hline 170 & 1 & 0 & 7.439567 & -1.866356 & 1.157510 \\
\hline 171 & 1 & 0 & 6.330387 & -1.993069 & 2.566642 \\
\hline 172 & 8 & 0 & 1.296443 & 2.823667 & -5.253281 \\
\hline 173 & 6 & 0 & 0.452716 & 3.945921 & -5.365508 \\
\hline 174 & 1 & 0 & -0.294269 & 3.702996 & -6.130529 \\
\hline 175 & 1 & 0 & -0.063324 & 4.161740 & -4.415113 \\
\hline 176 & 1 & 0 & 1.009777 & 4.843226 & -5.681393 \\
\hline
\end{tabular}

Compound $\boldsymbol{P} \mathbf{- 8}$, conformer 5

Center Atomic Atomic Coordinates (Angstroms) 


\begin{tabular}{cccccc} 
Number & Number & Type & X & Y & $\mathrm{Z}$ \\
\hline 1 & 8 & 0 & -3.963527 & -0.447300 & 4.519839 \\
2 & 6 & 0 & 1.187363 & 4.478969 & 2.193778 \\
3 & 1 & 0 & 2.219932 & 4.785307 & 2.409319 \\
4 & 1 & 0 & 0.639951 & 4.442854 & 3.150721 \\
5 & 6 & 0 & 1.189101 & 3.110313 & 1.534810 \\
6 & 1 & 0 & 0.159347 & 2.735969 & 1.417858 \\
7 & 1 & 0 & 1.615929 & 3.198047 & 0.520628 \\
8 & 6 & 0 & -2.711758 & -1.126147 & 4.534810 \\
9 & 1 & 0 & -2.448241 & -1.378426 & 5.574807 \\
10 & 1 & 0 & -1.918506 & -0.473260 & 4.143093 \\
11 & 6 & 0 & -2.813812 & -2.404461 & 3.730119 \\
12 & 1 & 0 & -1.876077 & -2.977995 & 3.863738 \\
13 & 1 & 0 & -3.641539 & -3.012413 & 4.124071 \\
14 & 6 & 0 & -2.701745 & 0.628491 & -3.906869 \\
15 & 1 & 0 & -2.402904 & 0.810839 & -4.949001 \\
16 & 1 & 0 & -2.129398 & 1.313736 & -3.260489 \\
17 & 6 & 0 & -2.419066 & -0.805208 & -3.499729 \\
18 & 1 & 0 & -2.920108 & -1.013976 & -2.546277 \\
19 & 1 & 0 & -2.828731 & -1.502229 & -4.255142 \\
20 & 8 & 0 & 1.944803 & 2.239326 & 2.343460 \\
21 & 8 & 0 & -3.085152 & -2.201798 & 2.366568 \\
22 & 8 & 0 & -1.022700 & -0.973285 & -3.384845 \\
23 & 6 & 0 & 3.256344 & 0.280788 & 0.617332 \\
24 & 6 & 0 & 1.812236 & 0.117299 & 1.095920 \\
25 & 6 & 0 & 1.000874 & -0.095013 & -1.130678 \\
26 & 6 & 0 & 2.386535 & 0.186764 & -1.727338 \\
27 & 6 & 0 & 3.468832 & -0.304151 & -0.773436 \\
28 & 1 & 0 & 1.634894 & -0.950982 & 1.307534 \\
29 & 1 & 0 & 3.487322 & 1.357915 & 0.571230 \\
30 & 1 & 0 & 0.205055 & 0.321295 & -1.758277 \\
31 & 1 & 0 & 2.477826 & 1.283625 & -1.806934 \\
32 & 1 & 0 & 3.403292 & -1.402646 & -0.704959 \\
33 & 8 & 0 & 0.896983 & 0.556928 & 0.104429 \\
34 & 8 & 0 & 0.843494 & -1.484741 & -0.993372 \\
35 & 8 & 0 & 2.585295 & -0.412841 & -2.981034 \\
36 & 8 & 0 & 4.755955 & 0.074523 & -1.208046 \\
37 & 8 & 0 & 4.095559 & -0.358730 & 1.553192 \\
38 & 6 & 0 & 1.995988 & 0.244509 & -4.093580 \\
39 & 1 & 0 & 2.257230 & -0.384248 & -4.957914 \\
40 & 6 & 0 & 5.480100 & -0.915923 & -1.896860 \\
41 & 1 & 0 & 5.640199 & -1.795669 & -1.245149 \\
42 & 1 & 0 & 4.903567 & -1.262849 & -2.771858 \\
43 & 6 & 0 & 5.235427 & 0.378498 & 1.966908 \\
& 1 & 0 & 4.911674 & 1.333011 & 2.418119 \\
\hline 7 & 6 & 0 & 5.868941 & 0.605248 & 1.094875 \\
& 6 & 0 & 6.813422 & -0.377316 & -2.347706 \\
& & 0 & 7.068307 & 0.994291 & -2.402287
\end{tabular}




\begin{tabular}{|c|c|c|c|c|c|}
\hline 49 & 6 & 0 & 9.042643 & -0.795618 & -3.206629 \\
\hline 50 & 1 & 0 & 7.631099 & -2.343897 & -2.705399 \\
\hline 51 & 6 & 0 & 8.300383 & 1.467285 & -2.854316 \\
\hline 52 & 1 & 0 & 6.291499 & 1.689606 & -2.084017 \\
\hline 53 & 6 & 0 & 9.290216 & 0.576097 & -3.259665 \\
\hline 54 & 1 & 0 & 9.814122 & -1.503772 & -3.517421 \\
\hline 55 & 1 & 0 & 8.486405 & 2.543353 & -2.888315 \\
\hline 56 & 1 & 0 & 10.254635 & 0.947238 & -3.613243 \\
\hline 57 & 6 & 0 & 5.994201 & -0.442750 & 2.972019 \\
\hline 58 & 6 & 0 & 5.696616 & -0.353308 & 4.334365 \\
\hline 59 & 6 & 0 & 6.965984 & -1.355151 & 2.552290 \\
\hline 60 & 6 & 0 & 6.357979 & -1.156769 & 5.260672 \\
\hline 61 & 1 & 0 & 4.938185 & 0.357362 & 4.672986 \\
\hline 62 & 6 & 0 & 7.629518 & -2.161032 & 3.474420 \\
\hline 63 & 1 & 0 & 7.206564 & -1.431746 & 1.488684 \\
\hline 64 & 6 & 0 & 7.325542 & -2.063670 & 4.831369 \\
\hline 65 & 1 & 0 & 6.118453 & -1.073752 & 6.323064 \\
\hline 66 & 1 & 0 & 8.389157 & -2.867696 & 3.133085 \\
\hline 67 & 1 & 0 & 7.846592 & -2.693106 & 5.556142 \\
\hline 68 & 6 & 0 & 1.538863 & 0.893127 & 2.373791 \\
\hline 69 & 1 & 0 & 2.103631 & 0.419275 & 3.187832 \\
\hline 70 & 1 & 0 & 0.460565 & 0.814743 & 2.605193 \\
\hline 71 & 6 & 0 & -0.410881 & -2.050186 & -1.265535 \\
\hline 72 & 8 & 0 & -1.436168 & -1.264058 & -0.701144 \\
\hline 73 & 6 & 0 & -2.287214 & -2.009986 & 0.159482 \\
\hline 74 & 6 & 0 & -1.982032 & -3.478940 & -0.144333 \\
\hline 75 & 6 & 0 & -0.510945 & -3.434915 & -0.568271 \\
\hline 76 & 1 & 0 & -3.329601 & -1.761529 & -0.090688 \\
\hline 77 & 1 & 0 & -2.107932 & -4.113135 & 0.748232 \\
\hline 78 & 1 & 0 & -0.289357 & -4.255046 & -1.271354 \\
\hline 79 & 6 & 0 & -0.635275 & -2.182937 & -2.781595 \\
\hline 80 & 1 & 0 & 0.315008 & -2.504618 & -3.232511 \\
\hline 81 & 1 & 0 & -1.393149 & -2.961291 & -2.960830 \\
\hline 82 & 8 & 0 & 0.276768 & -3.539542 & 0.583016 \\
\hline 83 & 8 & 0 & -2.730432 & -3.985449 & -1.222695 \\
\hline 84 & 6 & 0 & -2.046822 & -1.627651 & 1.611051 \\
\hline 85 & 1 & 0 & -2.041598 & -0.525540 & 1.684196 \\
\hline 86 & 1 & 0 & -1.062080 & -2.001168 & 1.941022 \\
\hline 87 & 6 & 0 & -4.098317 & -4.204410 & -0.961038 \\
\hline 88 & 1 & 0 & -4.663146 & -3.256166 & -0.991224 \\
\hline 89 & 1 & 0 & -4.215863 & -4.618964 & 0.059087 \\
\hline 90 & 1 & 0 & 0.897561 & 0.242425 & -4.011914 \\
\hline 91 & 6 & 0 & 2.503930 & 1.650977 & -4.312123 \\
\hline 92 & 6 & 0 & 1.615856 & 2.722439 & -4.418272 \\
\hline 93 & 6 & 0 & 3.877169 & 1.900443 & -4.411922 \\
\hline 94 & 6 & 0 & 2.085032 & 4.019046 & -4.631091 \\
\hline 95 & 1 & 0 & 0.541546 & 2.542066 & -4.327720 \\
\hline 96 & 6 & 0 & 4.349427 & 3.192536 & -4.618253 \\
\hline 97 & 1 & 0 & 4.581700 & 1.071167 & -4.317754 \\
\hline 98 & 6 & 0 & 3.453082 & 4.256616 & -4.73114 \\
\hline
\end{tabular}




$\begin{array}{cccccc}99 & 1 & 0 & 1.376245 & 4.846223 & -4.710896 \\ 100 & 1 & 0 & 5.424393 & 3.372189 & -4.692895 \\ 101 & 1 & 0 & 3.823827 & 5.270928 & -4.894712 \\ 102 & 6 & 0 & 2.169747 & -4.388688 & 1.747314 \\ 103 & 6 & 0 & 3.186339 & -3.602239 & 2.293107 \\ 104 & 6 & 0 & 1.681341 & -5.472295 & 2.485687 \\ 105 & 6 & 0 & 3.714378 & -3.898725 & 3.550355 \\ 106 & 1 & 0 & 3.567730 & -2.737719 & 1.745104 \\ 107 & 6 & 0 & 2.202650 & -5.767727 & 3.741364 \\ 108 & 1 & 0 & 0.884262 & -6.092106 & 2.066962 \\ 109 & 6 & 0 & 3.224030 & -4.981156 & 4.275777 \\ 110 & 1 & 0 & 4.510406 & -3.273877 & 3.962416 \\ 111 & 1 & 0 & 1.814845 & -6.618628 & 4.306008 \\ 112 & 1 & 0 & 3.636622 & -5.214510 & 5.259934 \\ 113 & 6 & 0 & 1.579245 & -4.071809 & 0.400141 \\ 114 & 1 & 0 & 2.212757 & -3.355946 & -0.143849 \\ 115 & 1 & 0 & 1.507932 & -4.991930 & -0.208506 \\ 116 & 6 & 0 & -4.677777 & -5.167511 & -1.966574 \\ 117 & 6 & 0 & -3.872933 & -6.088396 & -2.639722 \\ 118 & 6 & 0 & -6.055257 & -5.170704 & -2.206967 \\ 119 & 6 & 0 & -4.435111 & -6.995383 & -3.536564 \\ 120 & 1 & 0 & -2.797598 & -6.084888 & -2.458713 \\ 121 & 6 & 0 & -6.618933 & -6.081013 & -3.097411 \\ 122 & 1 & 0 & -6.695701 & -4.451727 & -1.688905 \\ 123 & 6 & 0 & -5.808888 & -6.996661 & -3.767255 \\ 124 & 1 & 0 & -3.792829 & -7.708149 & -4.058993 \\ 125 & 1 & 0 & -7.696825 & -6.071002 & -3.273802 \\ 126 & 1 & 0 & -6.248315 & -7.707748 & -4.470147 \\ 127 & 6 & 0 & -1.701583 & 5.211190 & 1.768399 \\ 128 & 6 & 0 & -3.012103 & 4.989450 & 1.327009 \\ 129 & 6 & 0 & -3.249389 & 4.884526 & -0.052959 \\ 130 & 6 & 0 & -2.167459 & 5.024763 & -0.930514 \\ 131 & 6 & 0 & -0.864356 & 5.210580 & -0.487703 \\ 132 & 6 & 0 & -0.627139 & 5.296959 & 0.892487 \\ 133 & 1 & 0 & -1.502775 & 5.304566 & 2.837695 \\ 134 & 1 & 0 & -2.320704 & 4.963559 & -2.009712 \\ 135 & 6 & 0 & -4.615860 & 4.596541 & -0.649867 \\ 136 & 1 & 0 & -4.704277 & 5.163780 & -1.586870 \\ 137 & 1 & 0 & -5.409849 & 4.972349 & 0.004532 \\ 138 & 6 & 0 & -4.096296 & 4.828443 & 2.379697 \\ 139 & 1 & 0 & -5.072008 & 5.124165 & 1.979345 \\ 140 & 1 & 0 & -3.882206 & 5.525746 & 3.201611 \\ 141 & 6 & 0 & -4.843005 & 3.128669 & -0.969370 \\ 142 & 6 & 0 & -5.456210 & 2.226738 & -0.081311 \\ 143 & 6 & 0 & -4.408455 & 2.651265 & -2.209866 \\ 144 & 6 & 0 & -5.626500 & 0.898609 & -0.486787 \\ 145 & 6 & 0 & -4.544181 & 1.321175 & -2.590283 \\ 146 & 1 & 0 & -3.942791 & 3.332054 & -2.925785 \\ 147 & 6 & 0 & -5.178775 & 0.427804 & -1.716258 \\ 148 & 1 & 0 & -6.126468 & 0.182597 & 0.168531\end{array}$




\begin{tabular}{|c|c|c|c|c|c|}
\hline 149 & 6 & 0 & -4.150023 & 3.422903 & 2.950890 \\
\hline 150 & 6 & 0 & -3.314212 & 3.124229 & 4.033439 \\
\hline 151 & 6 & 0 & -4.963287 & 2.402687 & 2.427649 \\
\hline 152 & 6 & 0 & -3.232194 & 1.851802 & 4.581501 \\
\hline 153 & 1 & 0 & -2.700286 & 3.903439 & 4.489733 \\
\hline 154 & 6 & 0 & -4.862579 & 1.117655 & 2.974341 \\
\hline 155 & 6 & 0 & -4.000523 & 0.819756 & 4.022236 \\
\hline 156 & 1 & 0 & -5.462654 & 0.296698 & 2.577473 \\
\hline 157 & 6 & 0 & -5.955856 & 2.622817 & 1.296658 \\
\hline 158 & 1 & 0 & -6.848244 & 2.020195 & 1.515969 \\
\hline 159 & 1 & 0 & -6.293835 & 3.664947 & 1.291284 \\
\hline 160 & 8 & 0 & 0.657242 & 5.470776 & 1.327966 \\
\hline 161 & 8 & 0 & 0.149647 & 5.241582 & -1.397616 \\
\hline 162 & 6 & 0 & 0.876505 & 6.454067 & -1.503977 \\
\hline 163 & 1 & 0 & 1.630469 & 6.305105 & -2.287552 \\
\hline 164 & 1 & 0 & 0.213433 & 7.284814 & -1.799487 \\
\hline 165 & 1 & 0 & 1.378427 & 6.706972 & -0.559244 \\
\hline 166 & 8 & 0 & -4.093145 & 0.908745 & -3.816598 \\
\hline 167 & 8 & 0 & -5.333604 & -0.895387 & -2.016075 \\
\hline 168 & 6 & 0 & -6.195967 & -1.197790 & -3.103217 \\
\hline 169 & 1 & 0 & -6.203130 & -2.289638 & -3.210726 \\
\hline 170 & 1 & 0 & -7.221542 & -0.850352 & -2.894800 \\
\hline 171 & 1 & 0 & -5.836702 & -0.740643 & -4.036040 \\
\hline 172 & 8 & 0 & -2.393219 & 1.619012 & 5.634235 \\
\hline 173 & 6 & 0 & -3.031390 & 1.366666 & 6.875448 \\
\hline 174 & 1 & 0 & -2.240755 & 1.191797 & 7.616086 \\
\hline 175 & 1 & 0 & -3.633735 & 2.234776 & 7.190982 \\
\hline 176 & 1 & 0 & -3.681726 & 0.479432 & 6.821603 \\
\hline
\end{tabular}

Compound $\boldsymbol{P} \mathbf{- 8}$, conformer 6

\begin{tabular}{cccccc} 
Center & Atomic & \multicolumn{2}{c}{ Atomic } & \multicolumn{2}{c}{ Coordinates } \\
Number & Number & Type & X & Y & Z \\
- & Numstroms & \\
1 & 8 & 0 & -3.947255 & -0.497412 & 4.525941 \\
2 & 6 & 0 & 1.178825 & 4.471493 & 2.207108 \\
3 & 1 & 0 & 2.211780 & 4.778689 & 2.419474 \\
4 & 1 & 0 & 0.636097 & 4.429728 & 3.166398 \\
5 & 6 & 0 & 1.180356 & 3.105824 & 1.541718 \\
6 & 1 & 0 & 0.150709 & 2.730559 & 1.426175 \\
7 & 1 & 0 & 1.604331 & 3.198504 & 0.526783 \\
8 & 6 & 0 & -2.694045 & -1.173798 & 4.532705 \\
9 & 1 & 0 & -2.429397 & -1.436109 & 5.569917 \\
10 & 1 & 0 & -1.902421 & -0.515287 & 4.147234 \\
11 & 6 & 0 & -2.793934 & -2.443956 & 3.715037 \\
12 & 1 & 0 & -1.855057 & -3.017049 & 3.842283 \\
13 & 1 & 0 & -3.620336 & -3.057537 & 4.102999 \\
14 & 6 & 0 & -2.705949 & 0.646422 & -3.892301 \\
15 & 1 & 0 & -2.407835 & 0.838644 & -4.932874
\end{tabular}




$\begin{array}{llllcc}16 & 1 & 0 & -2.134650 & 1.326872 & -3.239937 \\ 17 & 6 & 0 & -2.419924 & -0.790388 & -3.498560 \\ 18 & 1 & 0 & -2.916316 & -1.008072 & -2.544696 \\ 19 & 1 & 0 & -2.831966 & -1.481392 & -4.258157 \\ 20 & 8 & 0 & 1.939461 & 2.232484 & 2.344522 \\ 21 & 8 & 0 & -3.066270 & -2.227587 & 2.353788 \\ 22 & 8 & 0 & -1.022763 & -0.957306 & -3.391456 \\ 23 & 6 & 0 & 3.262856 & 0.284800 & 0.614572 \\ 24 & 6 & 0 & 1.817722 & 0.115460 & 1.087421 \\ 25 & 6 & 0 & 1.014513 & -0.088633 & -1.143166 \\ 26 & 6 & 0 & 2.402087 & 0.198162 & -1.733892 \\ 27 & 6 & 0 & 3.481860 & -0.294271 & -0.777647 \\ 28 & 1 & 0 & 1.641915 & -0.954179 & 1.293530 \\ 29 & 1 & 0 & 3.490532 & 1.362847 & 0.573217 \\ 30 & 1 & 0 & 0.219711 & 0.329585 & -1.770888 \\ 31 & 1 & 0 & 2.491214 & 1.295712 & -1.807376 \\ 32 & 1 & 0 & 3.418978 & -1.393205 & -0.713738 \\ 33 & 8 & 0 & 0.905480 & 0.557727 & 0.094431 \\ 34 & 8 & 0 & 0.858834 & -1.479383 & -1.013688 \\ 35 & 8 & 0 & 2.608914 & -0.394879 & -2.989104 \\ 36 & 8 & 0 & 4.769950 & 0.089786 & -1.204712 \\ 37 & 8 & 0 & 4.101156 & -0.355360 & 1.550782 \\ 38 & 6 & 0 & 1.997947 & 0.246243 & -4.101225 \\ 39 & 1 & 0 & 2.286322 & -0.367768 & -4.967132 \\ 40 & 6 & 0 & 5.498168 & -0.894177 & -1.900002 \\ 41 & 1 & 0 & 5.670173 & -1.773610 & -1.251223 \\ 42 & 1 & 0 & 4.918082 & -1.243008 & -2.771811 \\ 43 & 6 & 0 & 5.239930 & 0.382666 & 1.966354 \\ 44 & 1 & 0 & 4.914298 & 1.333742 & 2.423525 \\ 45 & 1 & 0 & 5.870750 & 0.616022 & 1.094200 \\ 46 & 6 & 0 & 6.822574 & -0.341340 & -2.359043 \\ 47 & 6 & 0 & 7.033394 & 1.033078 & -2.488376 \\ 48 & 6 & 0 & 7.858400 & -1.218002 & -2.698190 \\ 49 & 6 & 0 & 8.254906 & 1.520788 & -2.952059 \\ 50 & 1 & 0 & 6.230654 & 1.719193 & -2.217605 \\ 51 & 6 & 0 & 9.075892 & -0.732273 & -3.166510 \\ 52 & 1 & 0 & 7.709714 & -2.296147 & -2.592124 \\ 53 & 6 & 0 & 9.278781 & 0.641474 & -3.295073 \\ 54 & 1 & 0 & 8.406294 & 2.598825 & -3.044784 \\ 55 & 1 & 0 & 9.874527 & -1.430614 & -3.426803 \\ 56 & 1 & 0 & 10.235165 & 1.024231 & -3.657984 \\ 57 & 6 & 0 & 6.003028 & -0.442034 & 2.965312 \\ 58 & 6 & 0 & 5.703083 & -0.366198 & 4.327988 \\ 59 & 6 & 0 & 6.981441 & -1.344284 & 2.539142 \\ 60 & 6 & 0 & 6.368529 & -1.173141 & 5.248284 \\ 63 & 1 & 0 & 4.939546 & 0.336562 & 4.671610 \\ 65 & 1 & 0 & 7.649185 & -2.153545 & 3.455294 \\ & 1 & 0 & 6.127069 & -1.100830 & 6.311021\end{array}$




\begin{tabular}{|c|c|c|c|c|c|}
\hline 66 & 1 & 0 & 8.414009 & -2.852154 & 3.108989 \\
\hline 67 & 1 & 0 & 7.867002 & -2.701987 & 5.532669 \\
\hline 68 & 6 & 0 & 1.537845 & 0.884835 & 2.367767 \\
\hline 69 & 1 & 0 & 2.101745 & 0.409207 & 3.181380 \\
\hline 70 & 1 & 0 & 0.459122 & 0.802014 & 2.595584 \\
\hline 71 & 6 & 0 & -0.396487 & -2.044853 & -1.281860 \\
\hline 72 & 8 & 0 & -1.419639 & -1.263670 & -0.706901 \\
\hline 73 & 6 & 0 & -2.268844 & -2.017573 & 0.148677 \\
\hline 74 & 6 & 0 & -1.960878 & -3.483981 & -0.164952 \\
\hline 75 & 6 & 0 & -0.491014 & -3.433695 & -0.592456 \\
\hline 76 & 1 & 0 & -3.311942 & -1.769702 & -0.099217 \\
\hline 77 & 1 & 0 & -2.082454 & -4.123669 & 0.724273 \\
\hline 78 & 1 & 0 & -0.268911 & -4.249211 & -1.300703 \\
\hline 79 & 6 & 0 & -0.629804 & -2.169725 & -2.797274 \\
\hline 80 & 1 & 0 & 0.318059 & -2.487741 & -3.255932 \\
\hline 81 & 1 & 0 & -1.387552 & -2.948163 & -2.976299 \\
\hline 82 & 8 & 0 & 0.299769 & -3.542411 & 0.556352 \\
\hline 83 & 8 & 0 & -2.711353 & -3.986396 & -1.243766 \\
\hline 84 & 6 & 0 & -2.028889 & -1.645550 & 1.602997 \\
\hline 85 & 1 & 0 & -2.025650 & -0.544046 & 1.684927 \\
\hline 86 & 1 & 0 & -1.043481 & -2.019965 & 1.929983 \\
\hline 87 & 6 & 0 & -4.077773 & -4.210584 & -0.978759 \\
\hline 88 & 1 & 0 & -4.645209 & -3.263694 & -1.001647 \\
\hline 89 & 1 & 0 & -4.190726 & -4.631167 & 0.039403 \\
\hline 90 & 1 & 0 & 0.900391 & 0.203058 & -4.020933 \\
\hline 91 & 6 & 0 & 2.451351 & 1.672333 & -4.310063 \\
\hline 92 & 6 & 0 & 3.806617 & 1.964176 & -4.500999 \\
\hline 93 & 6 & 0 & 1.531454 & 2.722138 & -4.307362 \\
\hline 94 & 6 & 0 & 4.229759 & 3.276142 & -4.687962 \\
\hline 95 & 1 & 0 & 4.537172 & 1.151742 & -4.497371 \\
\hline 96 & 6 & 0 & 1.950722 & 4.038993 & -4.498168 \\
\hline 97 & 1 & 0 & 0.471613 & 2.508278 & -4.146752 \\
\hline 98 & 6 & 0 & 3.301083 & 4.318328 & -4.688708 \\
\hline 99 & 1 & 0 & 5.290946 & 3.489002 & -4.836026 \\
\hline 100 & 1 & 0 & 1.217184 & 4.848006 & -4.488084 \\
\hline 101 & 1 & 0 & 3.633228 & 5.348342 & -4.836405 \\
\hline 102 & 6 & 0 & 2.195759 & -4.396809 & 1.711959 \\
\hline 103 & 6 & 0 & 3.208386 & -3.609424 & 2.263790 \\
\hline 104 & 6 & 0 & 1.713548 & -5.489066 & 2.441578 \\
\hline 105 & 6 & 0 & 3.738636 & -3.913461 & 3.518281 \\
\hline 106 & 1 & 0 & 3.584844 & -2.738357 & 1.722785 \\
\hline 107 & 6 & 0 & 2.237064 & -5.792081 & 3.694539 \\
\hline 108 & 1 & 0 & 0.919638 & -6.109727 & 2.018110 \\
\hline 109 & 6 & 0 & 3.254469 & -5.004488 & 4.234951 \\
\hline 110 & 1 & 0 & 4.531518 & -3.287806 & 3.935144 \\
\hline 111 & 1 & 0 & 1.854126 & -6.649704 & 4.252290 \\
\hline 112 & 1 & 0 & 3.668837 & -5.243786 & 5.216933 \\
\hline 113 & 6 & 0 & 1.602686 & -4.071765 & 0.367864 \\
\hline 114 & 1 & 0 & 2.234314 & -3.351475 & -0.172461 \\
\hline 115 & 1 & 0 & 1.531609 & -4.987929 & -0.246711 \\
\hline
\end{tabular}




$\begin{array}{llllll}116 & 6 & 0 & -4.658047 & -5.169529 & -1.987779 \\ 117 & 6 & 0 & -3.853036 & -6.084276 & -2.669039 \\ 118 & 6 & 0 & -6.036360 & -5.175176 & -2.223346 \\ 119 & 6 & 0 & -4.415862 & -6.987650 & -3.569126 \\ 120 & 1 & 0 & -2.777080 & -6.078827 & -2.491788 \\ 121 & 6 & 0 & -6.600639 & -6.081909 & -3.117039 \\ 122 & 1 & 0 & -6.676951 & -4.460987 & -1.698882 \\ 123 & 6 & 0 & -5.790428 & -6.991422 & -3.795004 \\ 124 & 1 & 0 & -3.773449 & -7.695597 & -4.097908 \\ 125 & 1 & 0 & -7.679169 & -6.073894 & -3.289597 \\ 126 & 1 & 0 & -6.230372 & -7.699659 & -4.500445 \\ 127 & 6 & 0 & -1.711467 & 5.189064 & 1.800654 \\ 128 & 6 & 0 & -3.023665 & 4.967682 & 1.364427 \\ 129 & 6 & 0 & -3.269182 & 4.880978 & -0.015371 \\ 130 & 6 & 0 & -2.194533 & 5.045365 & -0.897741 \\ 131 & 6 & 0 & -0.889889 & 5.234819 & -0.460815 \\ 132 & 6 & 0 & -0.643326 & 5.295305 & 0.919222 \\ 133 & 1 & 0 & -1.506386 & 5.265985 & 2.870042 \\ 134 & 1 & 0 & -2.355103 & 5.002358 & -1.976772 \\ 135 & 6 & 0 & -4.636700 & 4.587296 & -0.606871 \\ 136 & 1 & 0 & -4.734580 & 5.159990 & -1.539588 \\ 137 & 1 & 0 & -5.429912 & 4.952651 & 0.054377 \\ 138 & 6 & 0 & -4.101200 & 4.791996 & 2.421659 \\ 139 & 1 & 0 & -5.080117 & 5.087430 & 2.028987 \\ 140 & 1 & 0 & -3.885426 & 5.483635 & 3.247928 \\ 141 & 6 & 0 & -4.854864 & 3.119732 & -0.935050 \\ 142 & 6 & 0 & -5.460854 & 2.207996 & -0.052033 \\ 143 & 6 & 0 & -4.419571 & 2.653136 & -2.179494 \\ 144 & 6 & 0 & -5.624888 & 0.881855 & -0.466601 \\ 145 & 6 & 0 & -4.548841 & 1.325030 & -2.568937 \\ 146 & 1 & 0 & -3.958558 & 3.341292 & -2.891376 \\ 147 & 6 & 0 & -5.177418 & 0.422215 & -1.700257 \\ 148 & 1 & 0 & -6.119552 & 0.158569 & 0.184723 \\ 149 & 6 & 0 & -4.147629 & 3.382297 & 2.982626 \\ 150 & 6 & 0 & -3.306458 & 3.078280 & 4.059475 \\ 151 & 6 & 0 & -4.961172 & 2.363968 & 2.456241 \\ 152 & 6 & 0 & -3.219646 & 1.802482 & 4.598882 \\ 153 & 1 & 0 & -2.692339 & 3.855775 & 4.518440 \\ 154 & 6 & 0 & -4.855768 & 1.075618 & 2.994139 \\ 155 & 6 & 0 & -3.988495 & 0.772607 & 4.036326 \\ 156 & 1 & 0 & -5.456248 & 0.256147 & 2.594834 \\ 157 & 6 & 0 & -5.958441 & 2.590116 & 1.330603 \\ 158 & 1 & 0 & -6.847644 & 1.981872 & 1.547270 \\ 159 & 1 & 0 & -6.300648 & 3.630867 & 1.335520 \\ 160 & 8 & 0 & 0.643385 & 5.466530 & 1.348379 \\ 161 & 8 & 0 & 0.116822 & 5.295302 & -1.377411 \\ 162 & 6 & 0 & 0.836842 & 6.514130 & -1.455882 \\ 163 & 1 & 0 & 1.586046 & 6.390378 & -2.248230 \\ 164 & 1 & 0 & 0.167419 & 7.348965 & -1.724246 \\ 165 & 1 & 0 & 1.344155 & 6.743876 & -0.508237\end{array}$




$\begin{array}{llllll}166 & 8 & 0 & -4.097708 & 0.923445 & -3.798842 \\ 167 & 8 & 0 & -5.326466 & -0.899479 & -2.009520 \\ 168 & 6 & 0 & -6.189987 & -1.197888 & -3.096869 \\ 169 & 1 & 0 & -6.192241 & -2.288927 & -3.212502 \\ 170 & 1 & 0 & -7.216684 & -0.856877 & -2.883452 \\ 171 & 1 & 0 & -5.835095 & -0.732097 & -4.027095 \\ 172 & 8 & 0 & -2.375882 & 1.564630 & 5.646672 \\ 173 & 6 & 0 & -3.008481 & 1.303831 & 6.888997 \\ 174 & 1 & 0 & -2.214505 & 1.125457 & 7.625212 \\ 175 & 1 & 0 & -3.610612 & 2.169192 & 7.212386 \\ 176 & 1 & 0 & -3.657888 & 0.416085 & 6.832440\end{array}$




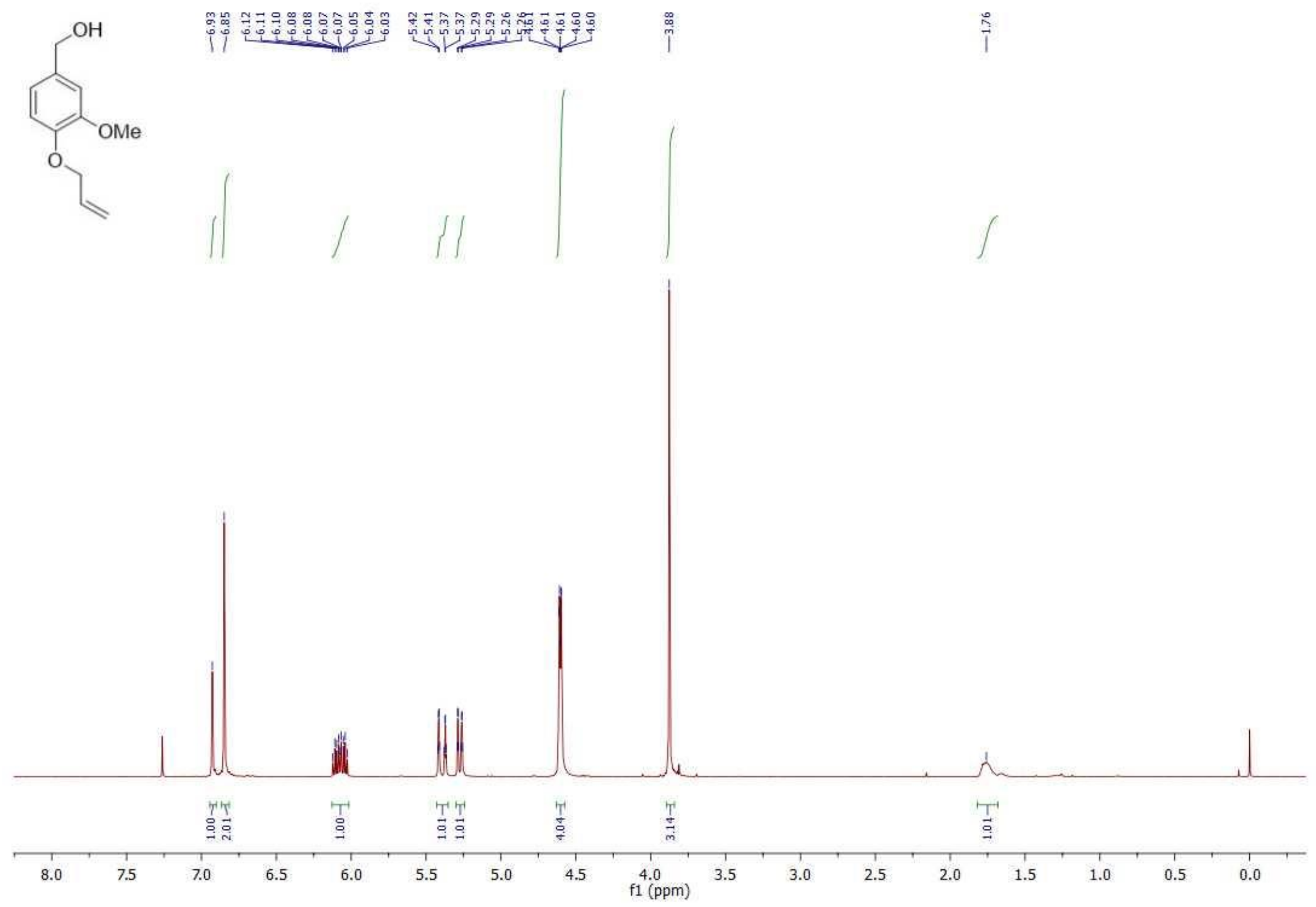

Figure S2. ${ }^{1} \mathrm{H}$ NMR spectrum of compound S1. 

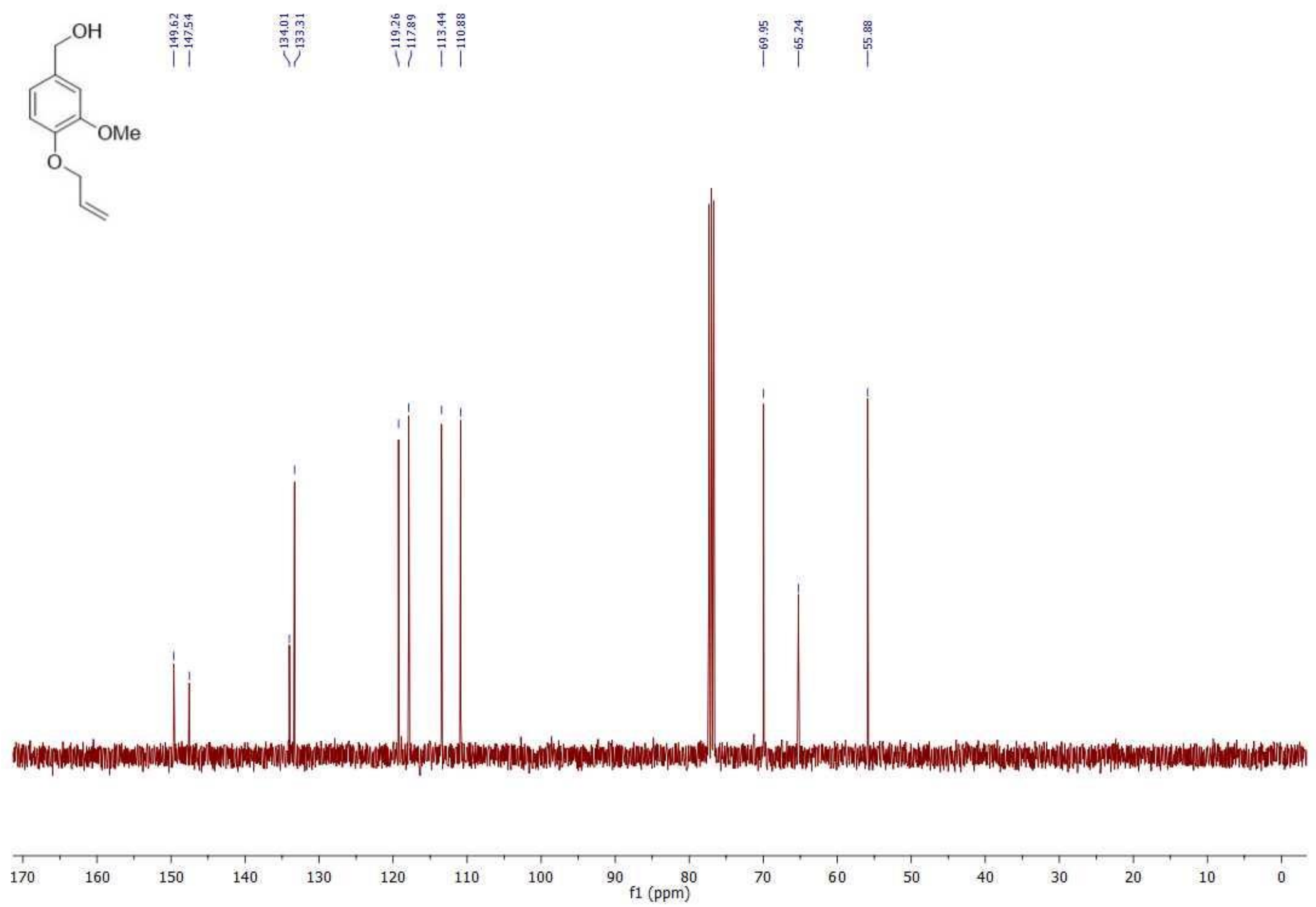

Figure S3. ${ }^{13} \mathrm{C}$ NMR spectrum of compound $\mathbf{S 1}$. 


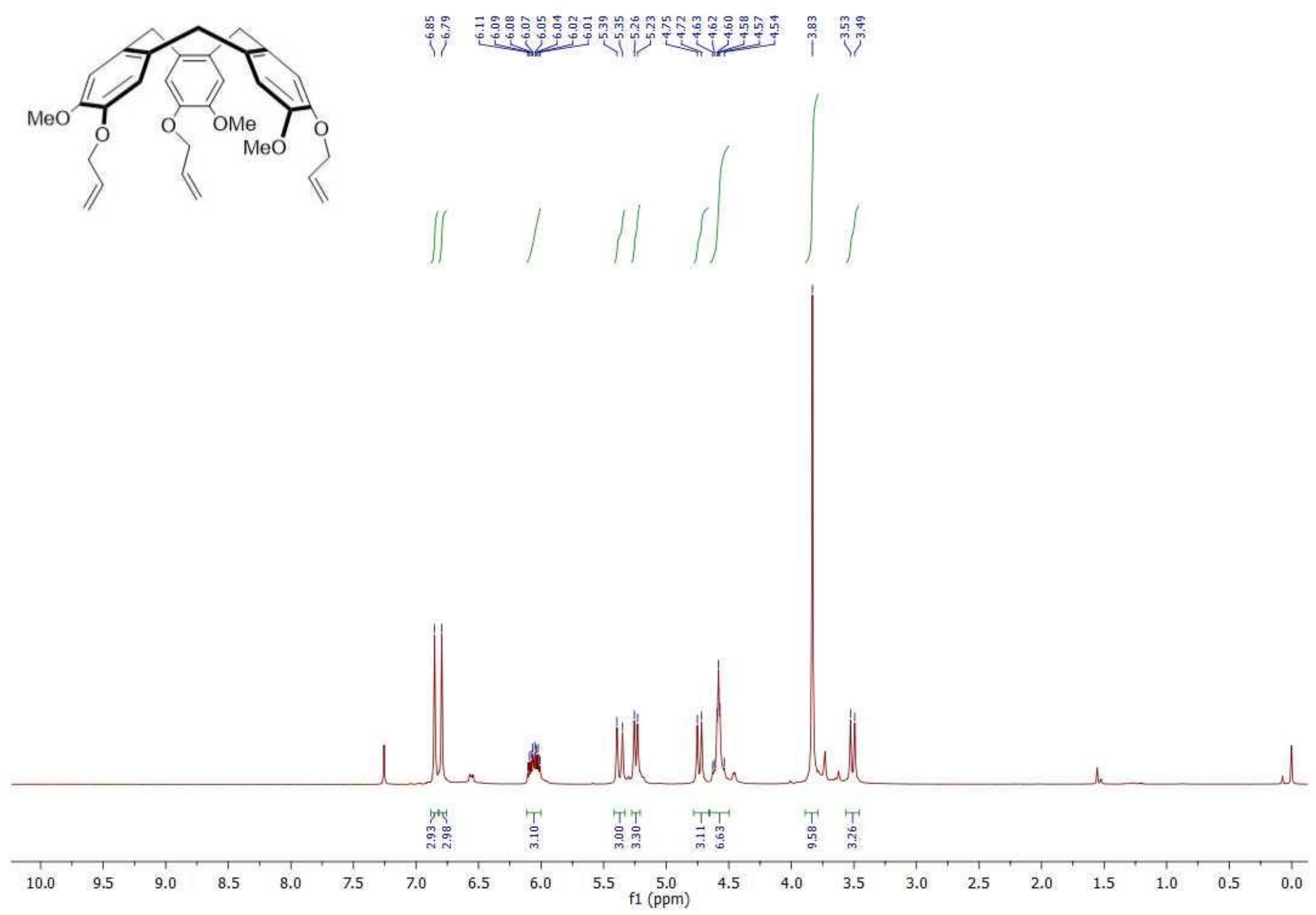

Figure S4. ${ }^{1} \mathrm{H}$ NMR spectrum of compound rac-S2. 


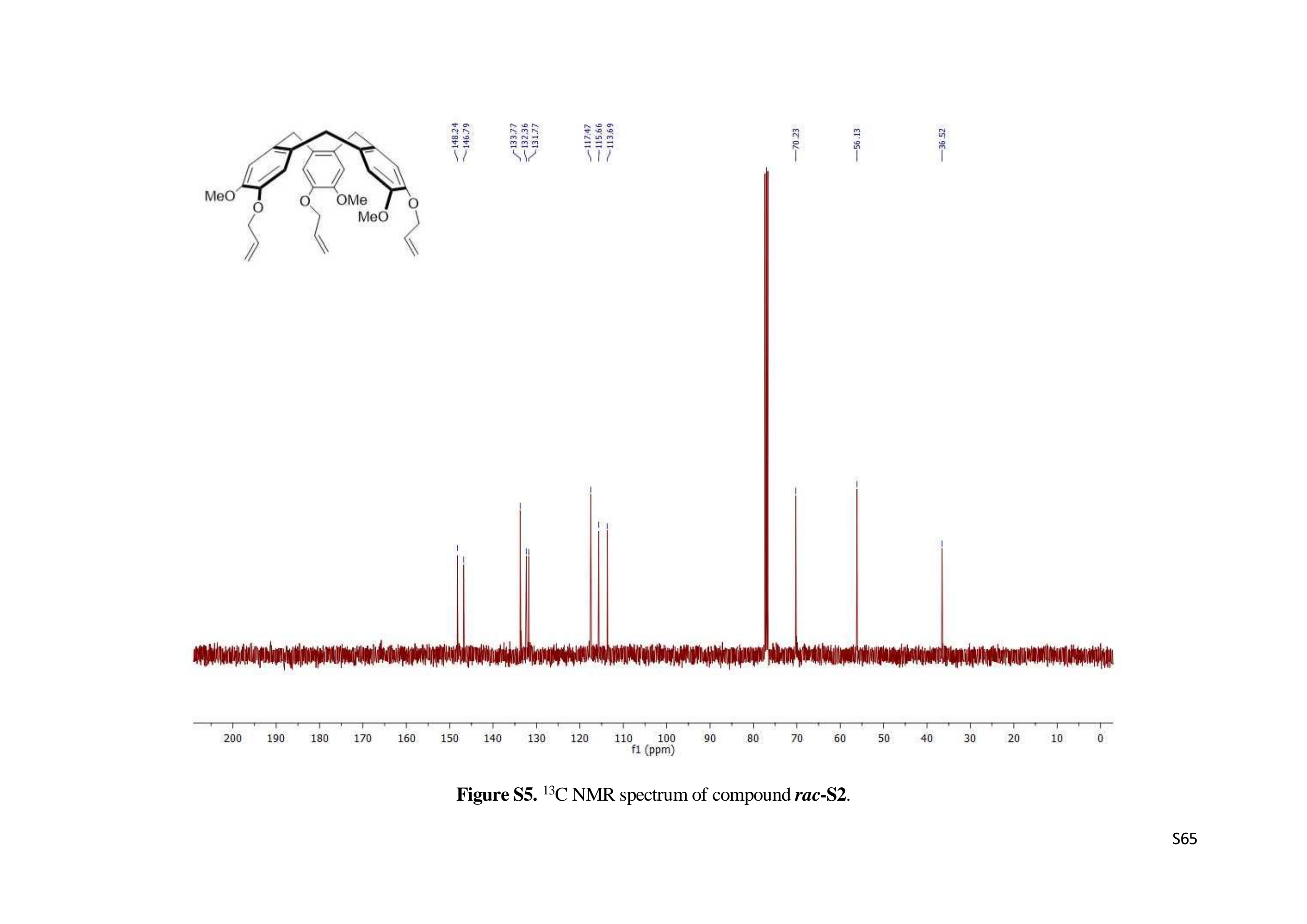




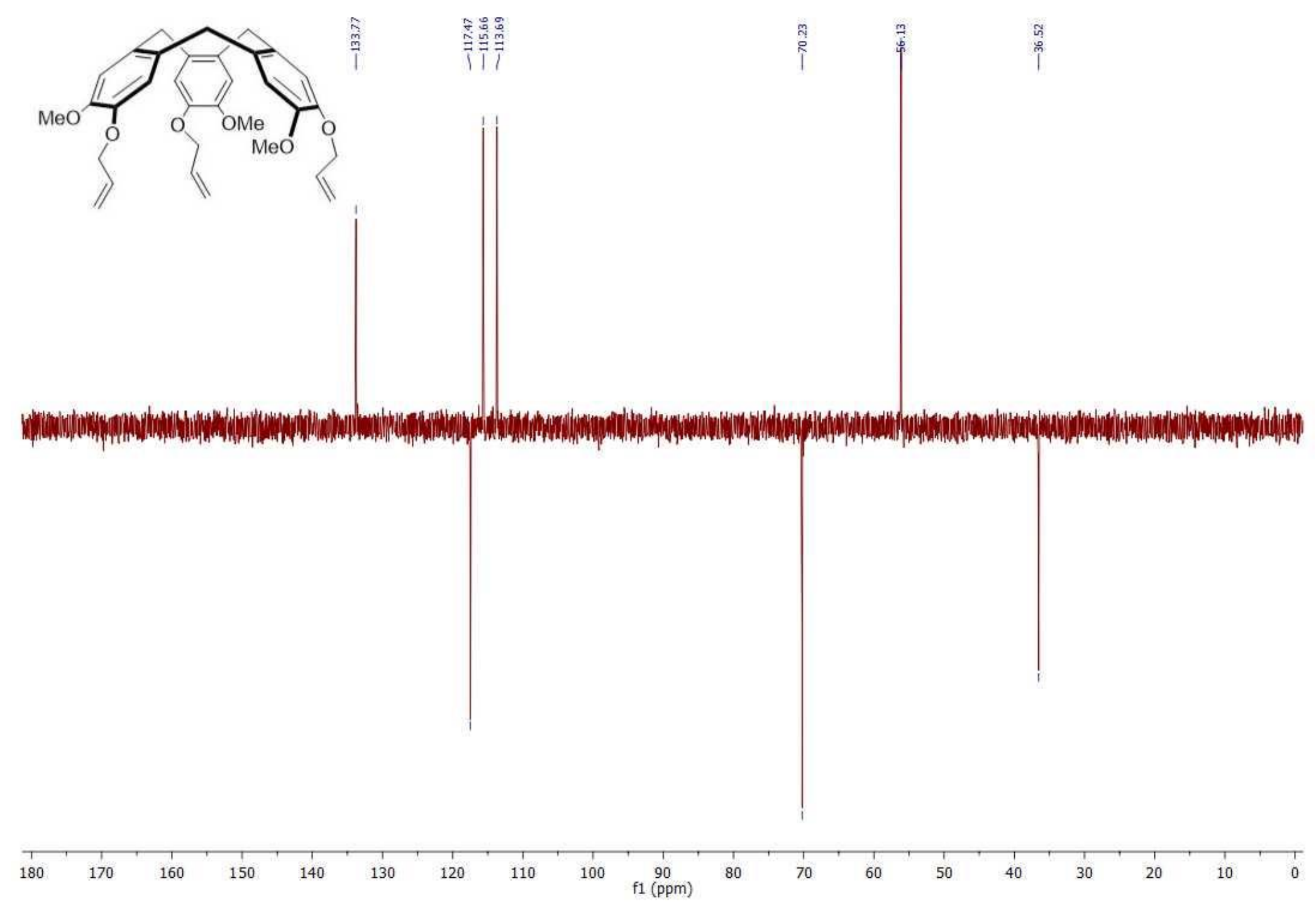

Figure S6. DEPT ${ }^{13} \mathrm{C}$ NMR spectrum of compound rac-S2. 


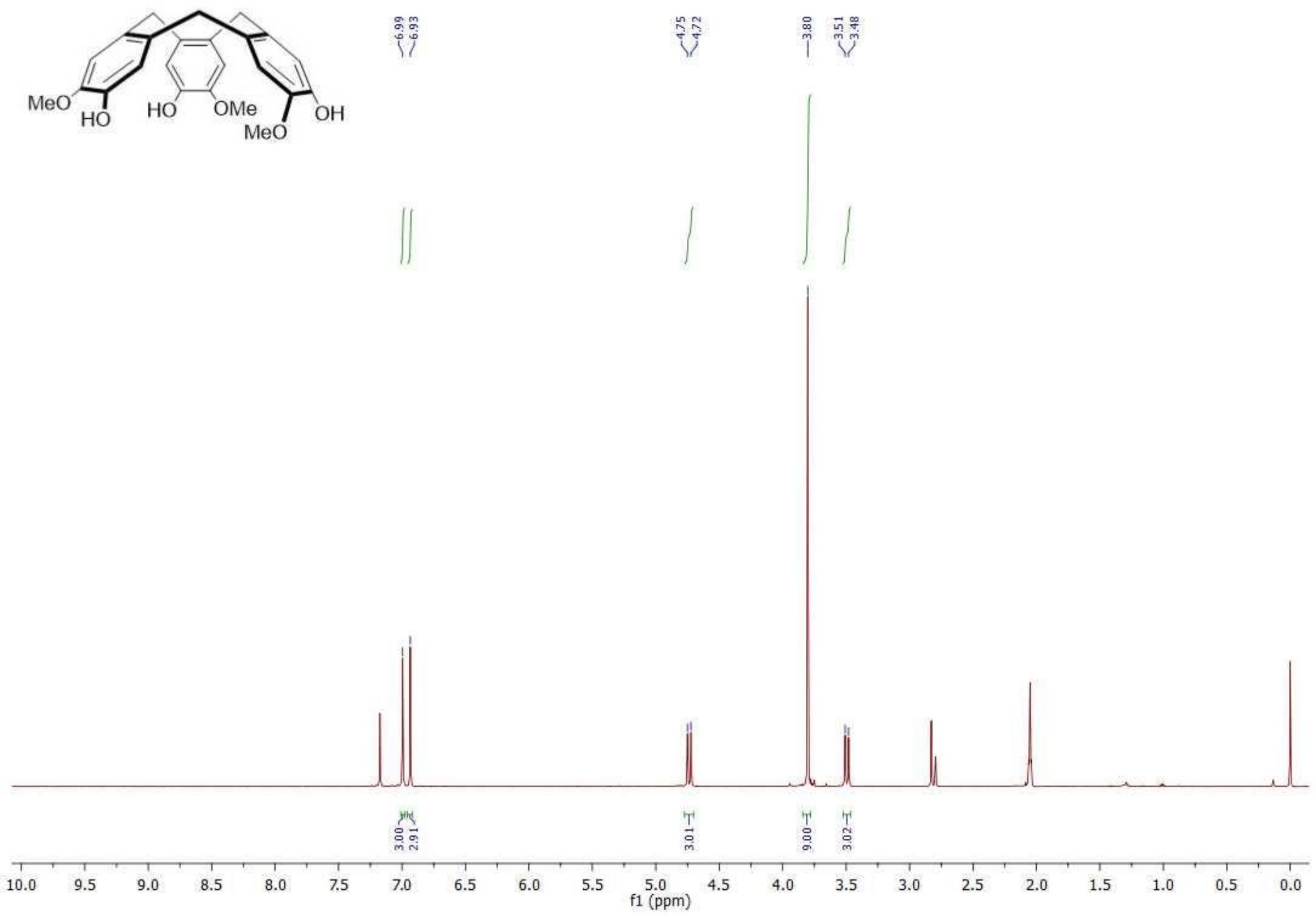

Figure S7. ${ }^{1} \mathrm{H}$ NMR spectrum of compound rac-7. 


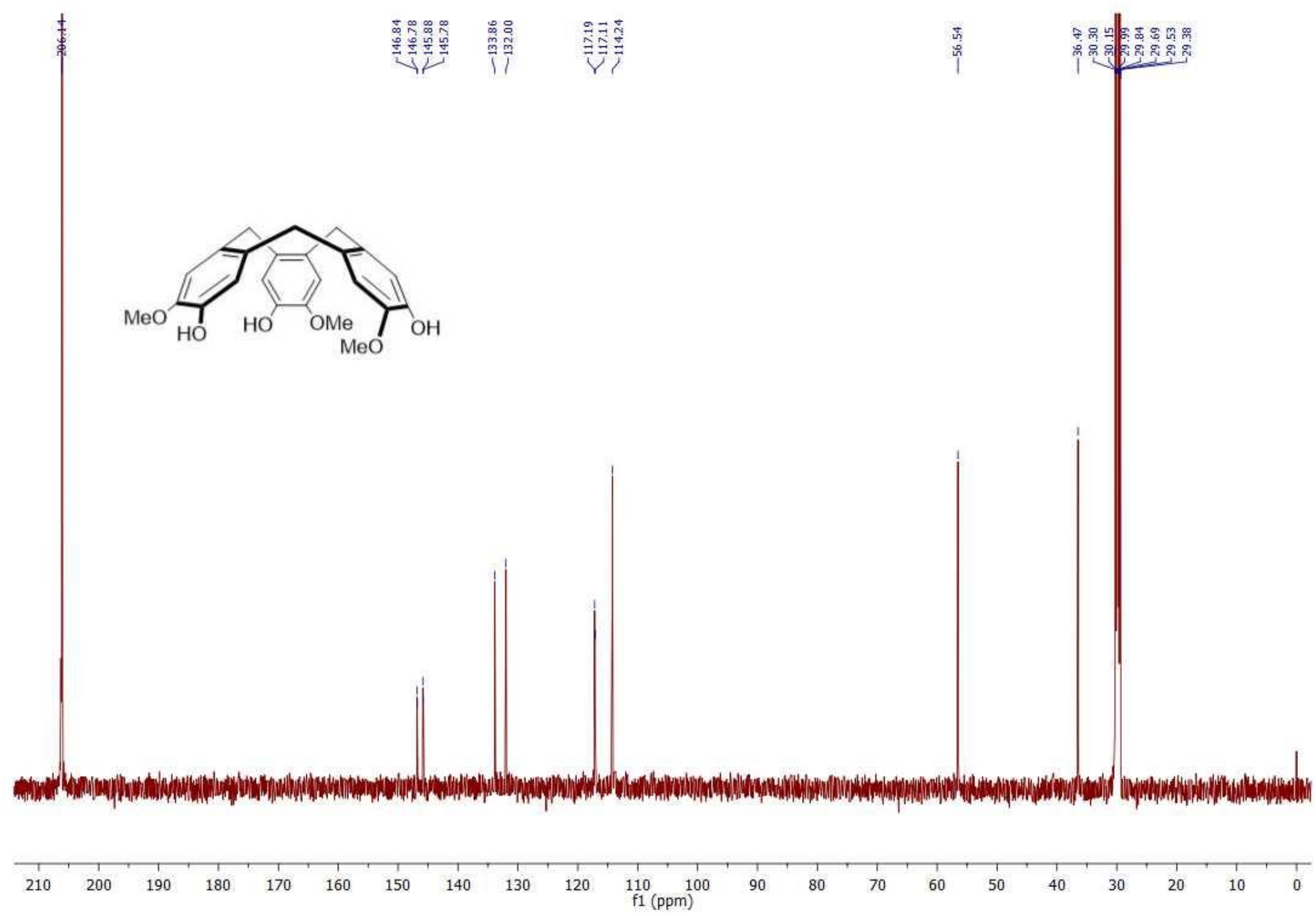

Figure S8. ${ }^{13} \mathrm{C}$ NMR spectrum of compound rac-7. 


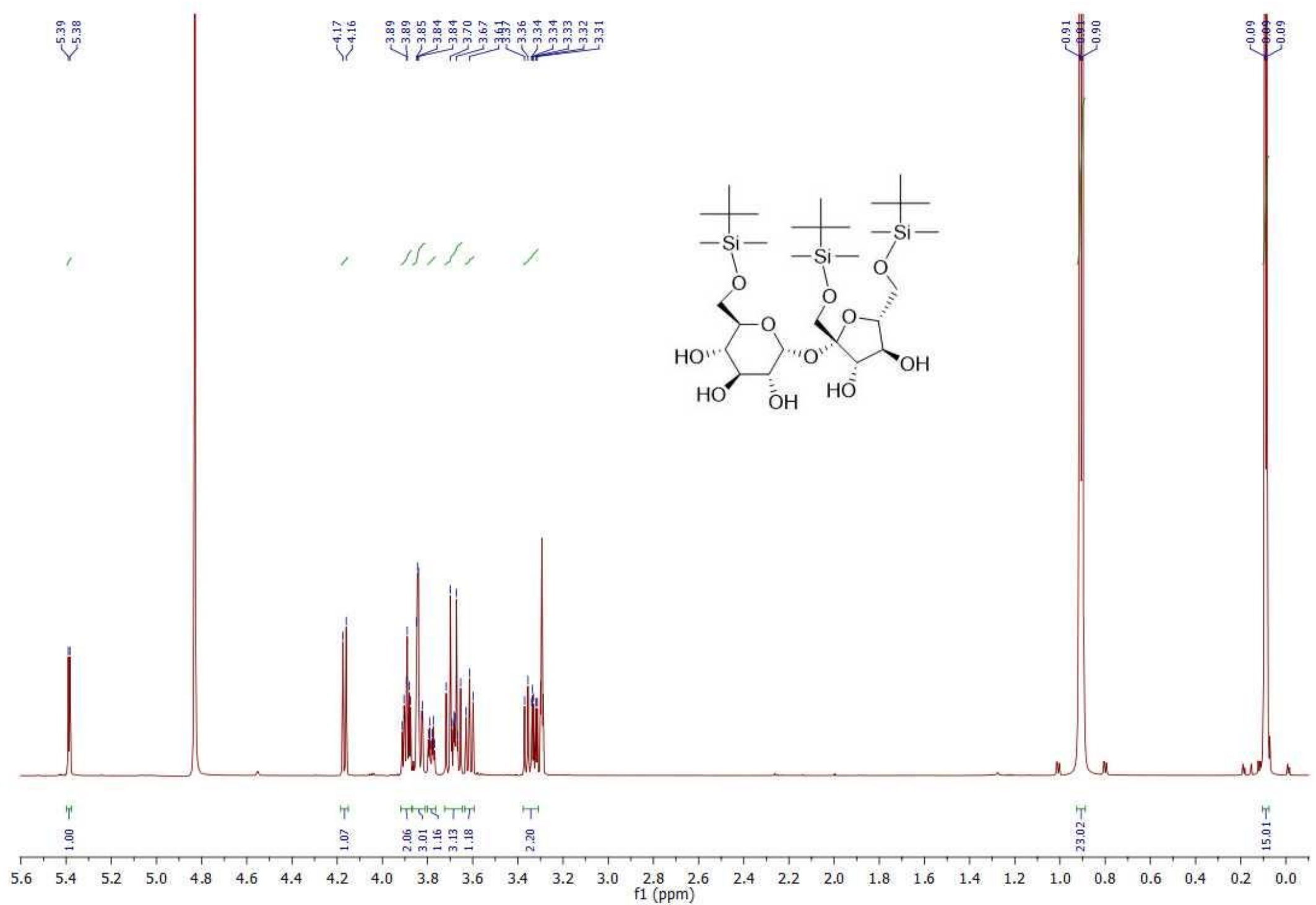

Figure S9. ${ }^{1} \mathrm{H}$ NMR spectrum of compound S3. 
(1)

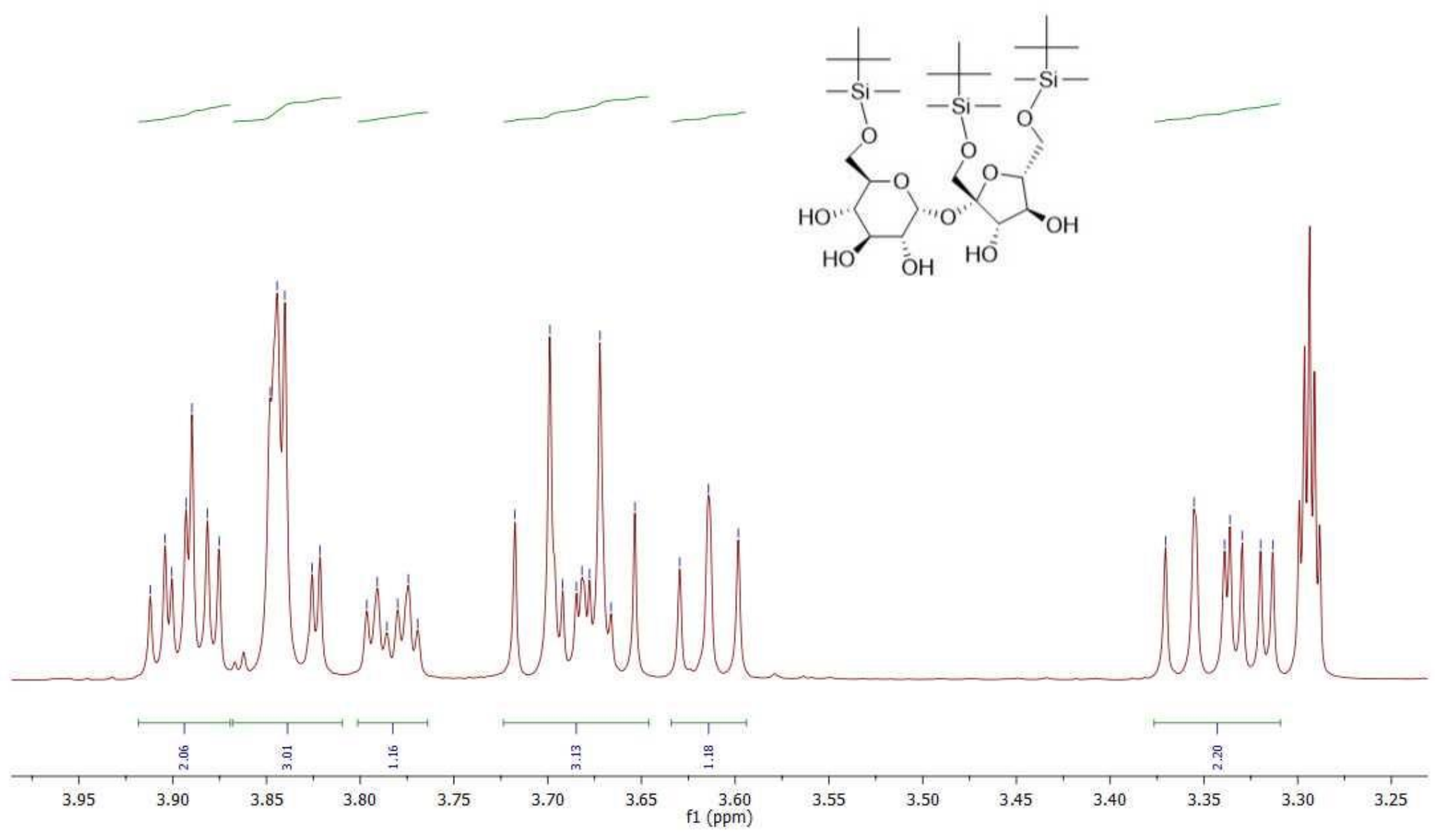

Figure S10. ${ }^{1} \mathrm{H}$ NMR spectrum of compound $\mathbf{S 3}$ (aliphatic part). 


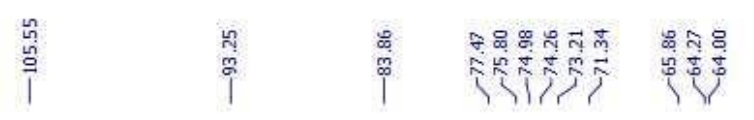
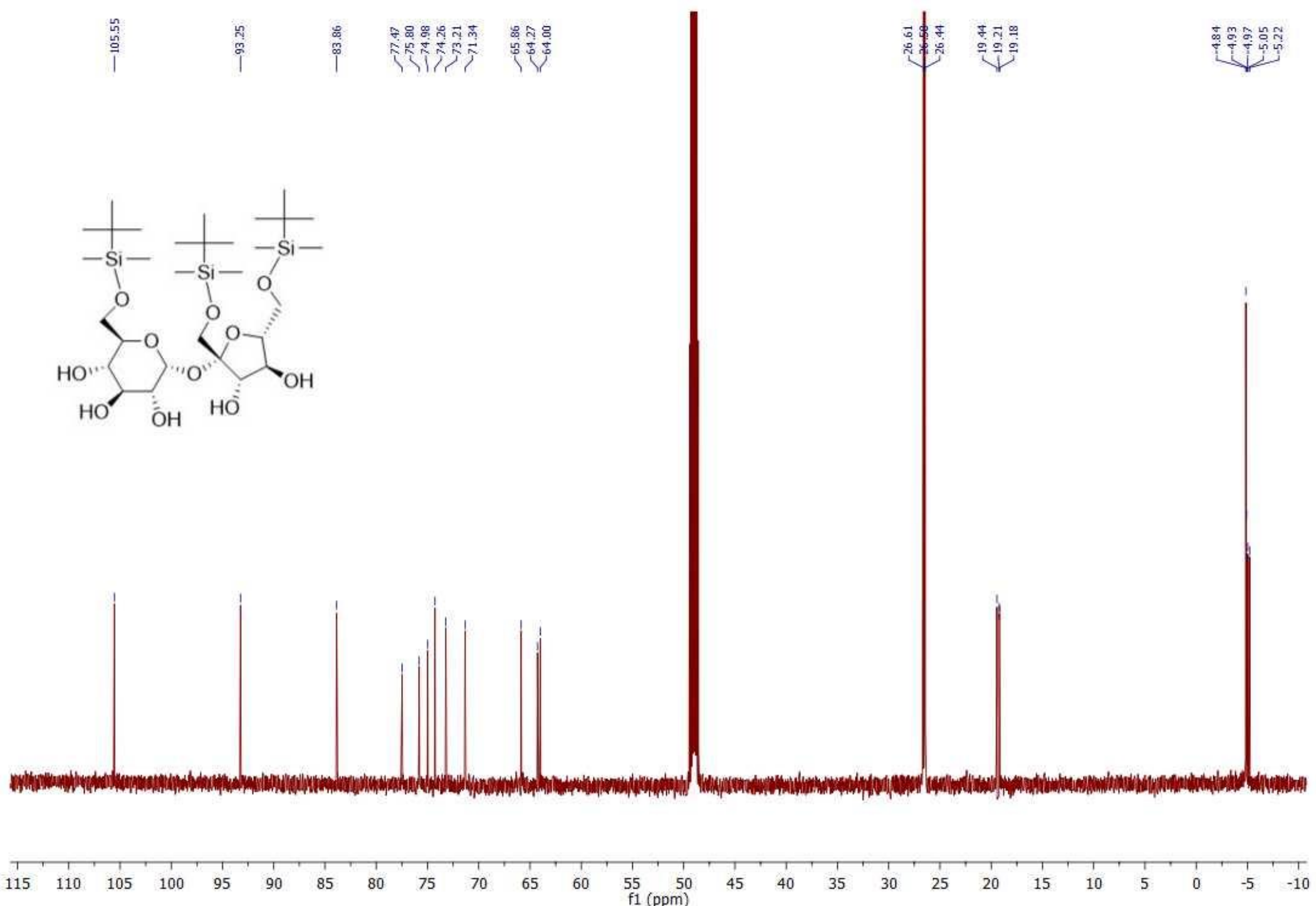

Figure S11. ${ }^{13} \mathrm{C}$ NMR spectrum of compound S3. 


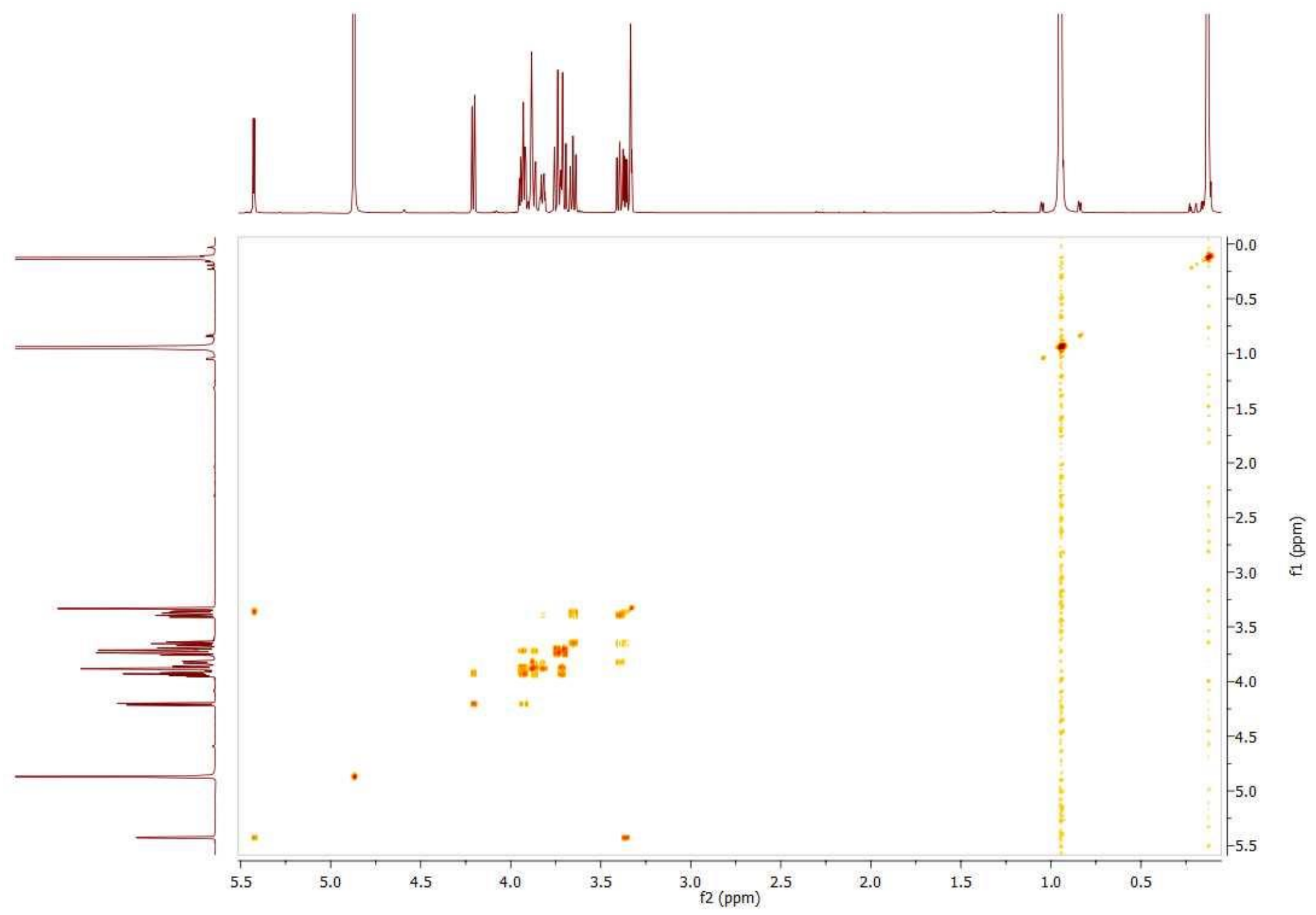

Figure S12. ${ }^{1} \mathrm{H}-{ }^{1} \mathrm{H}$ COSY spectrum of compound S3. 


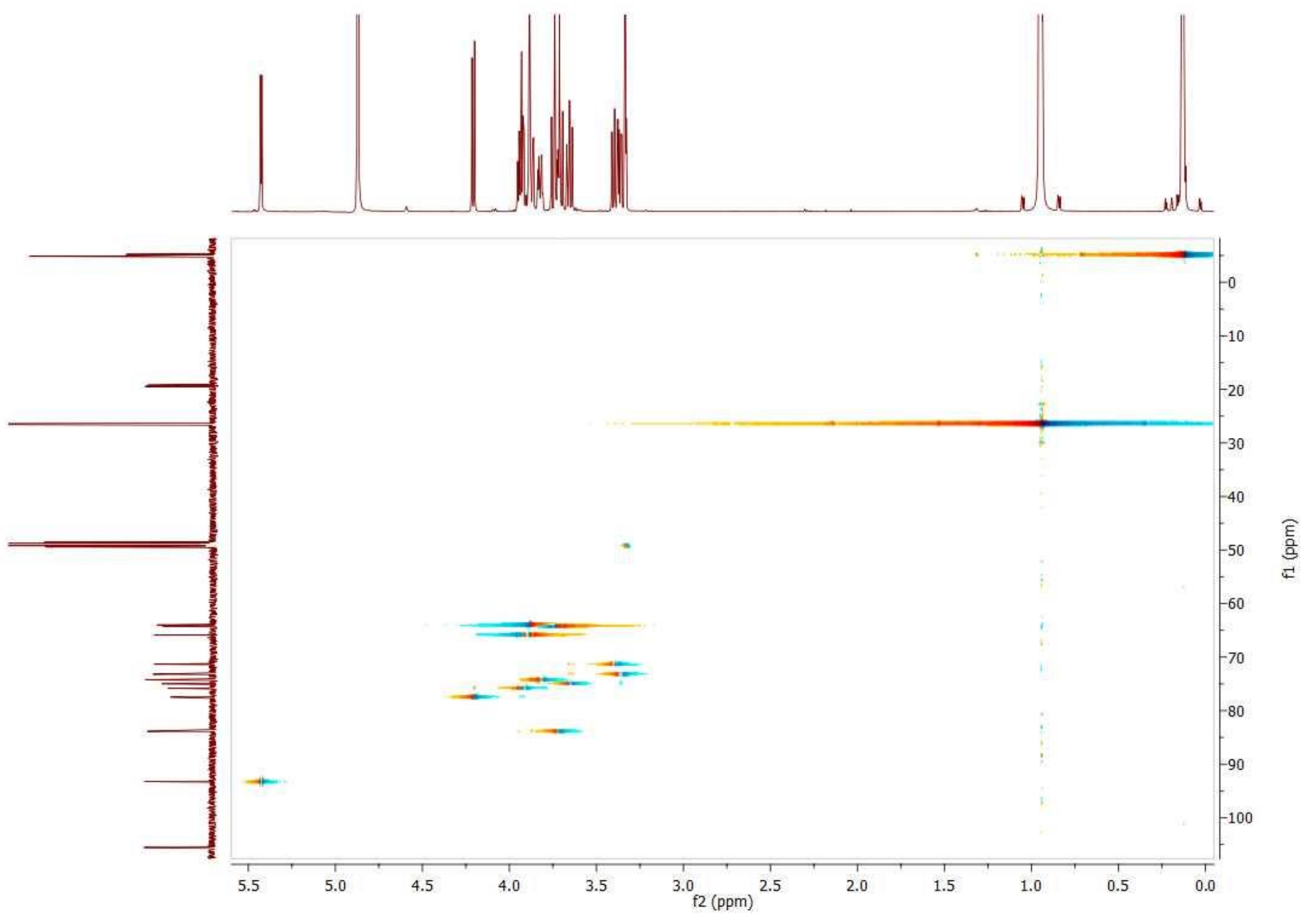

Figure S13. ${ }^{1} \mathrm{H}-{ }^{13} \mathrm{C}$ HSQC spectrum of compound $\mathbf{S 3}$. 


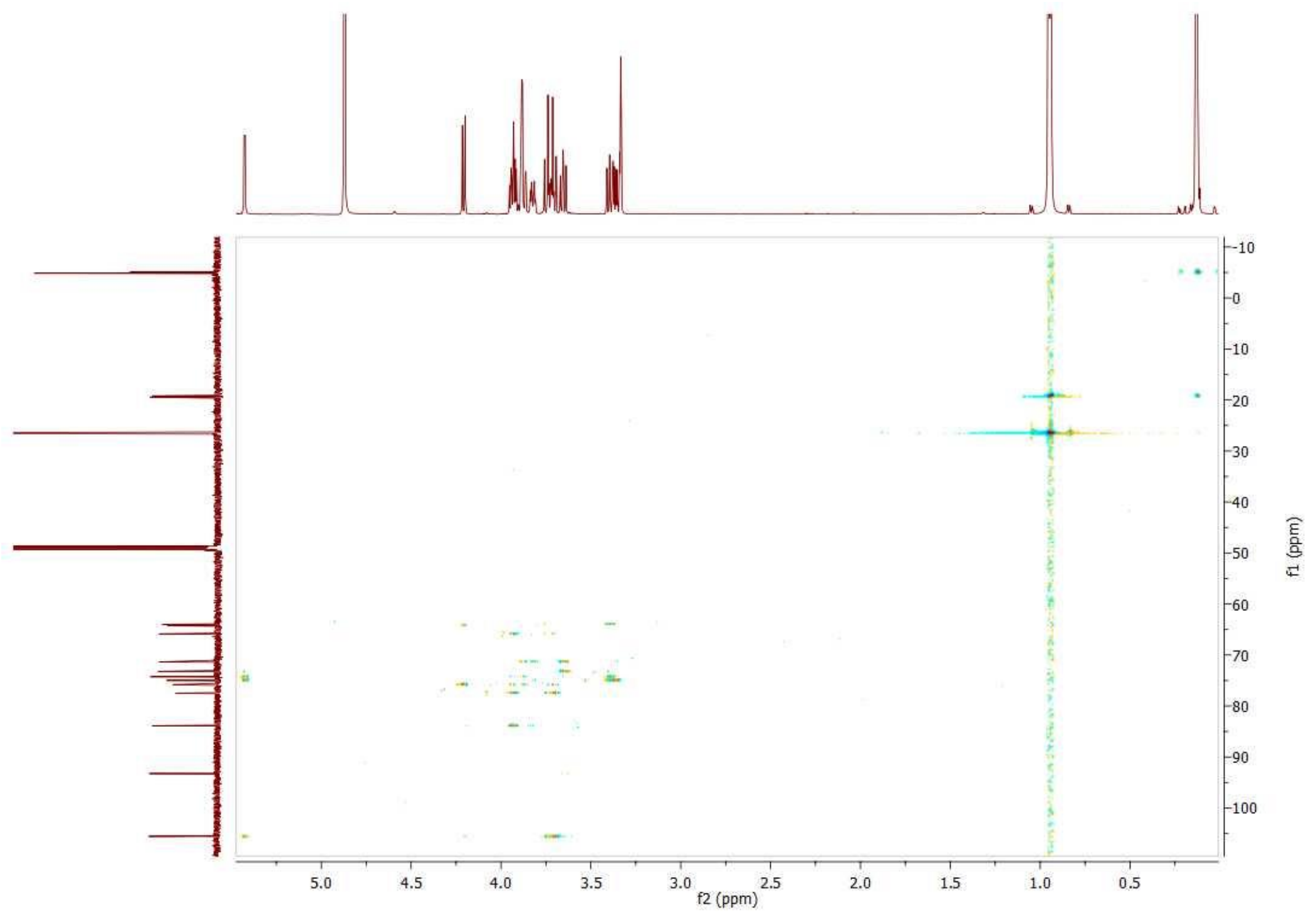

Figure S14. ${ }^{1} \mathrm{H}-{ }^{13} \mathrm{C}$ HMBC spectrum of compound S3. 


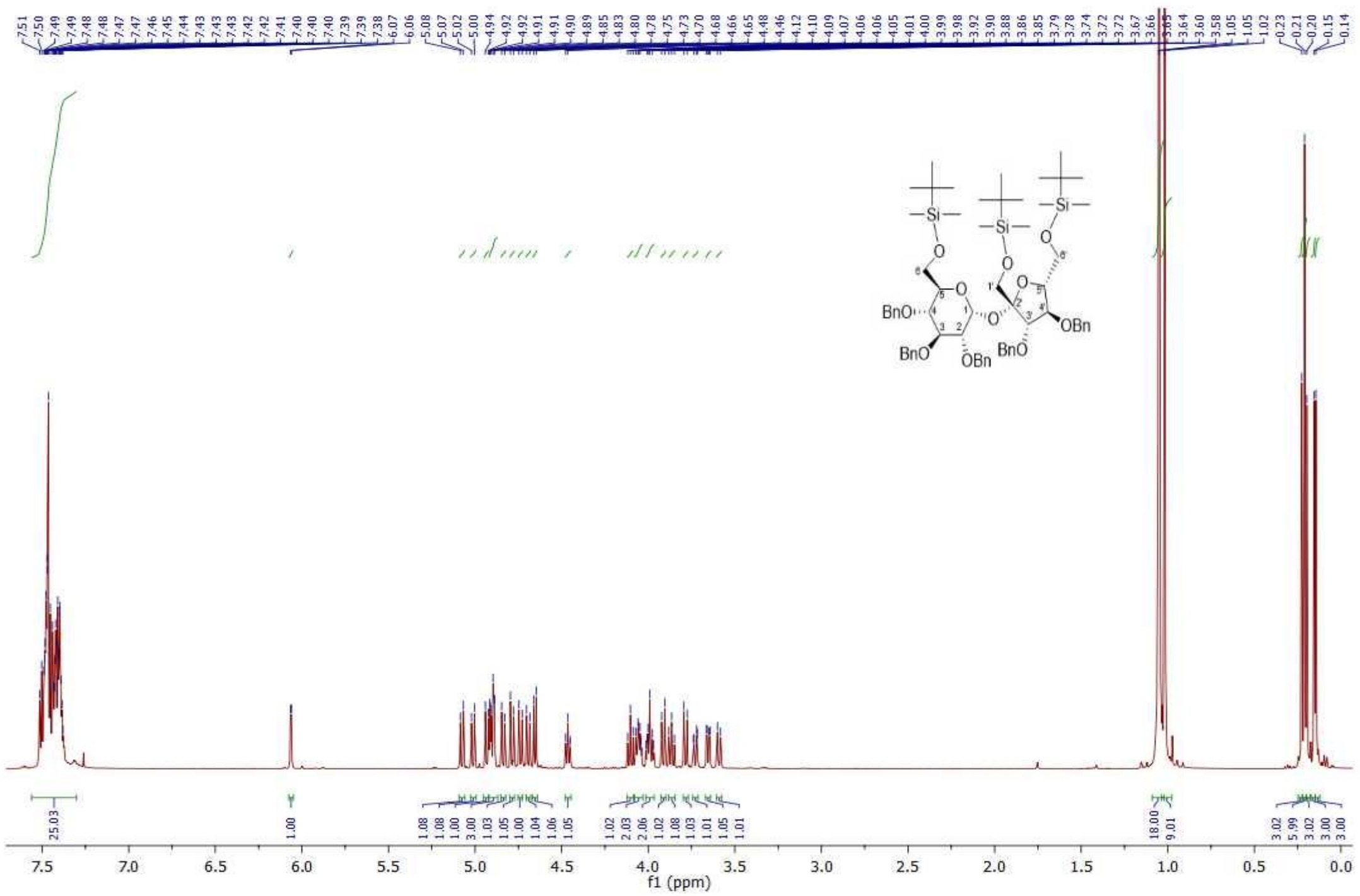

Figure S15. ${ }^{1} \mathrm{H}$ NMR spectrum of compound 2. 


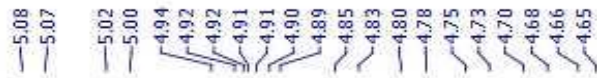

$\ldots \ldots \ldots$

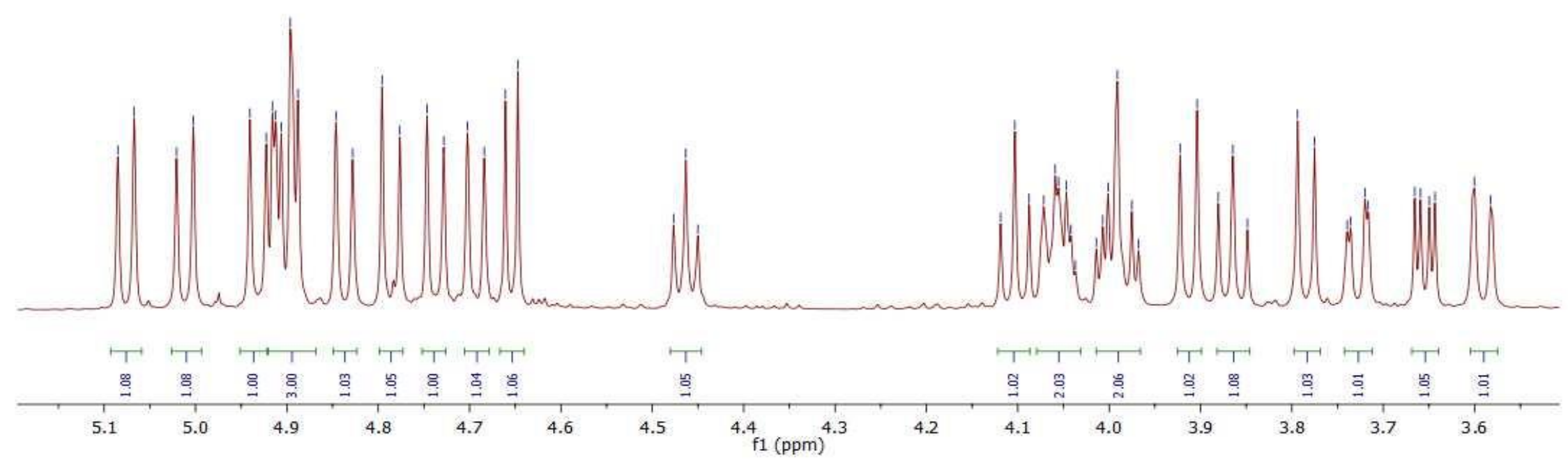

Figure S16. ${ }^{1} \mathrm{H}$ NMR spectrum of compound 2 (aliphatic part). 

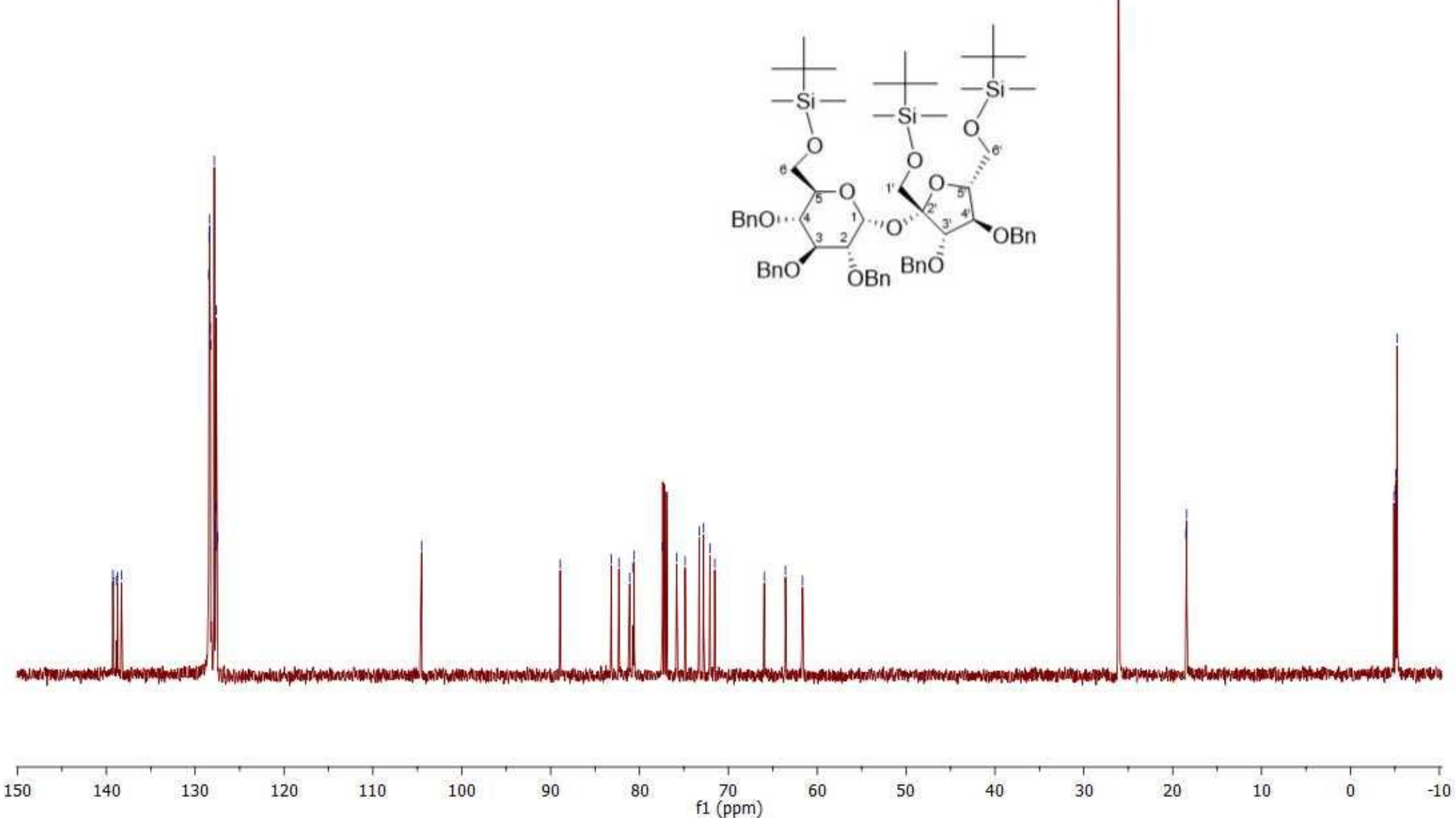

Figure S17. ${ }^{13} \mathrm{C}$ NMR spectrum of compound 2. 


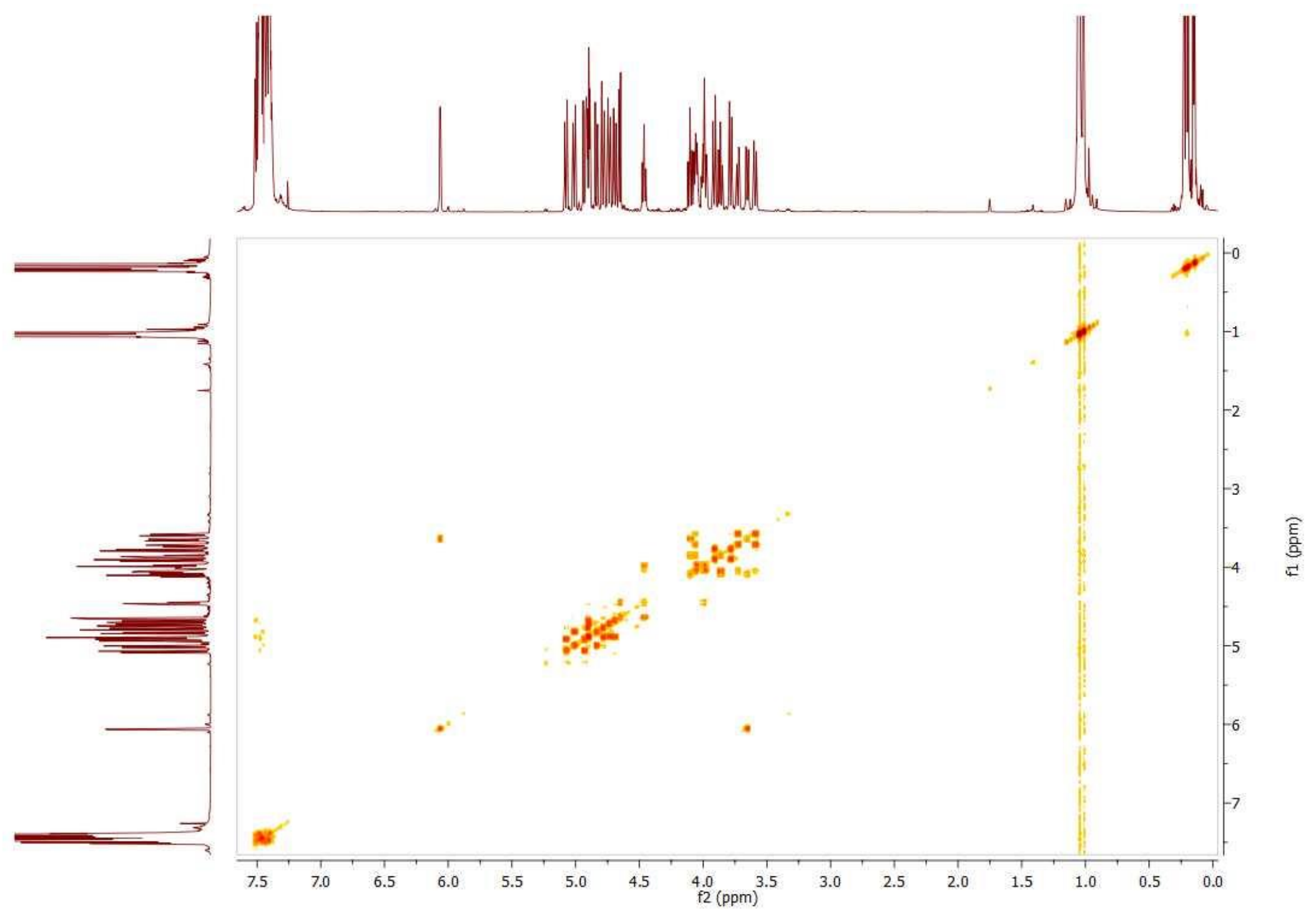

Figure S18. ${ }^{1} \mathrm{H}-{ }^{1} \mathrm{H}$ COSY spectrum of compound 2. 


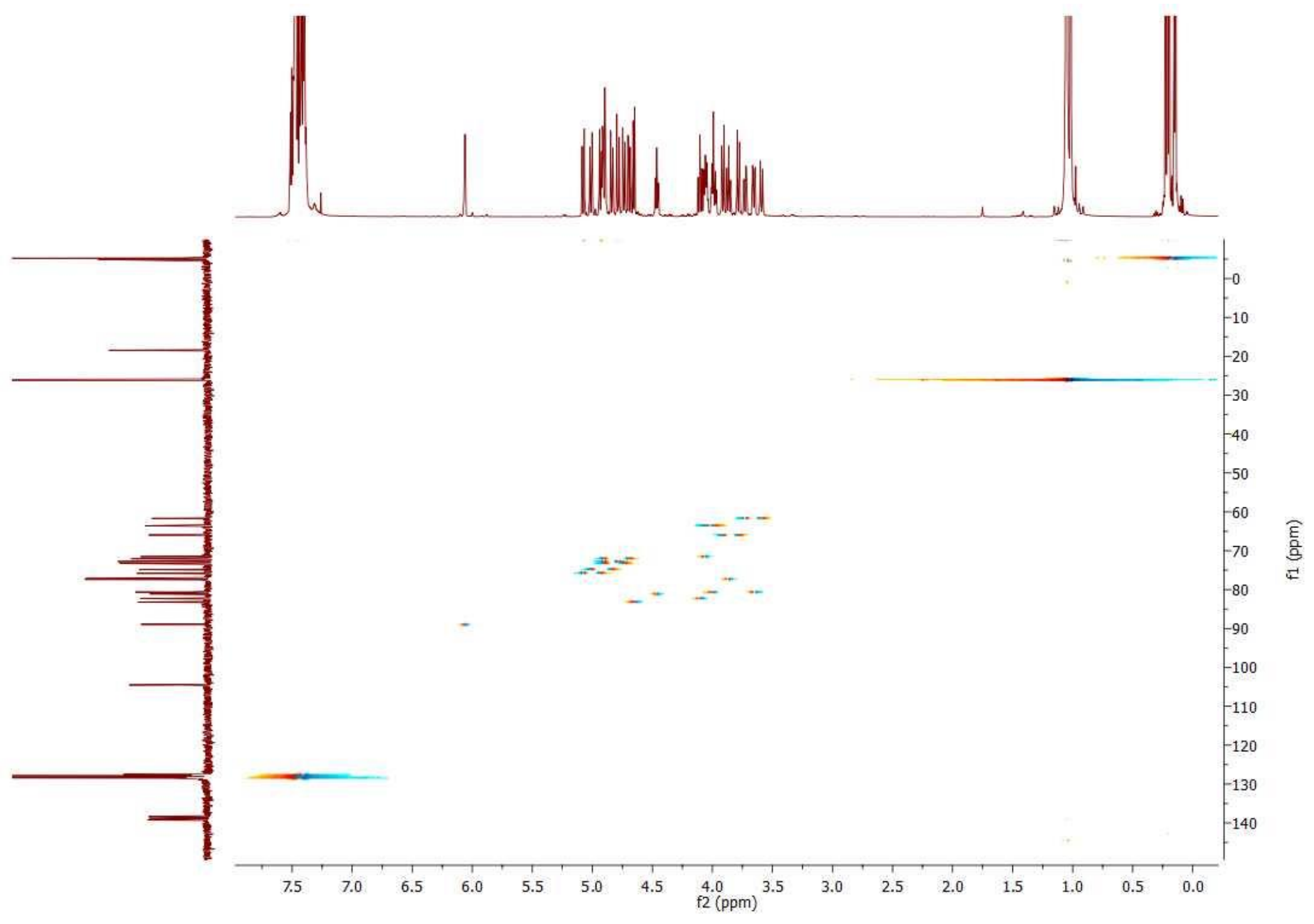

Figure S19. ${ }^{1} \mathrm{H}-{ }^{13} \mathrm{C}$ HSQC spectrum of compound 2. 


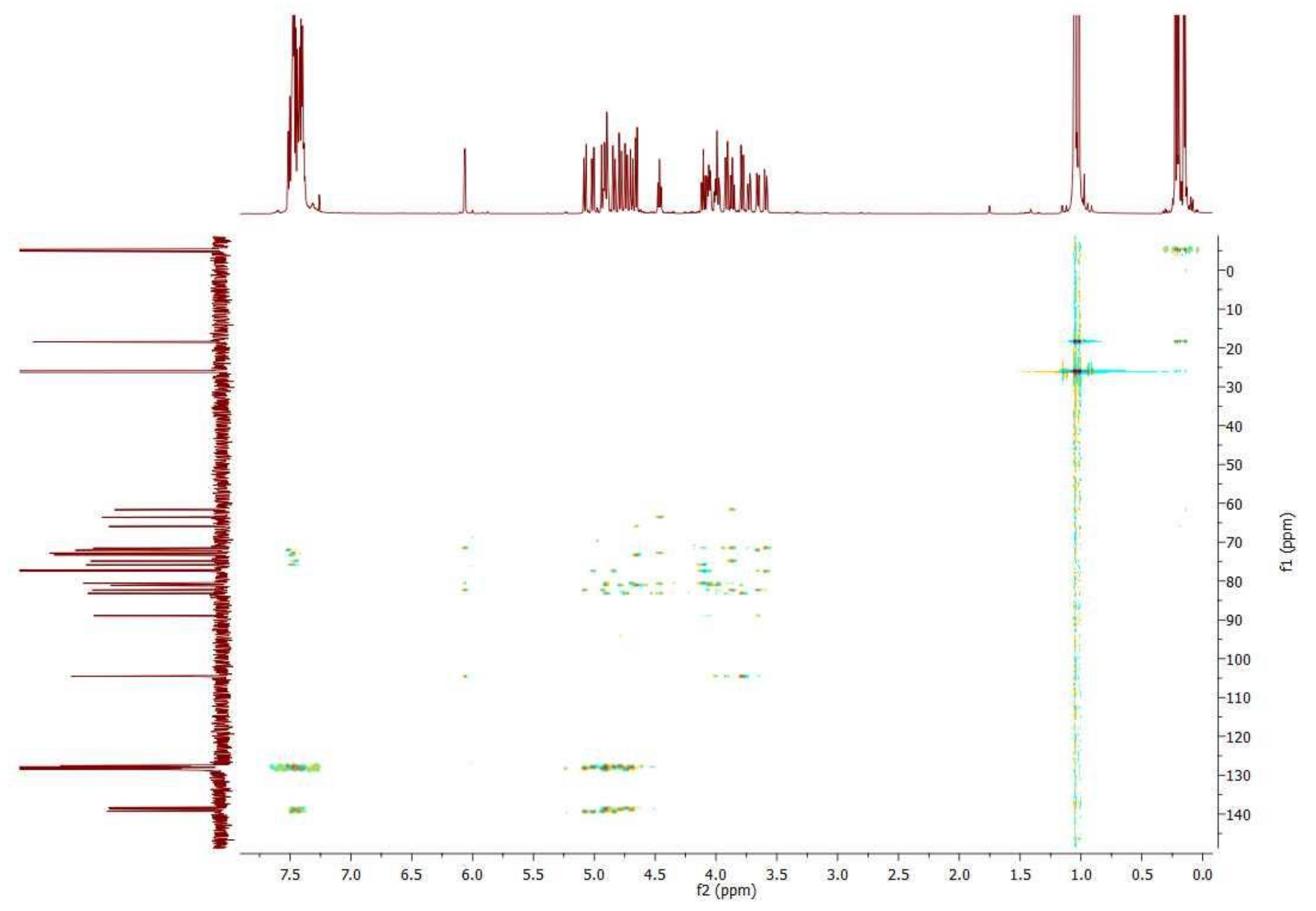

Figure S20. ${ }^{1} \mathrm{H}-{ }^{13} \mathrm{C}$ HMBC spectrum of compound 2. 


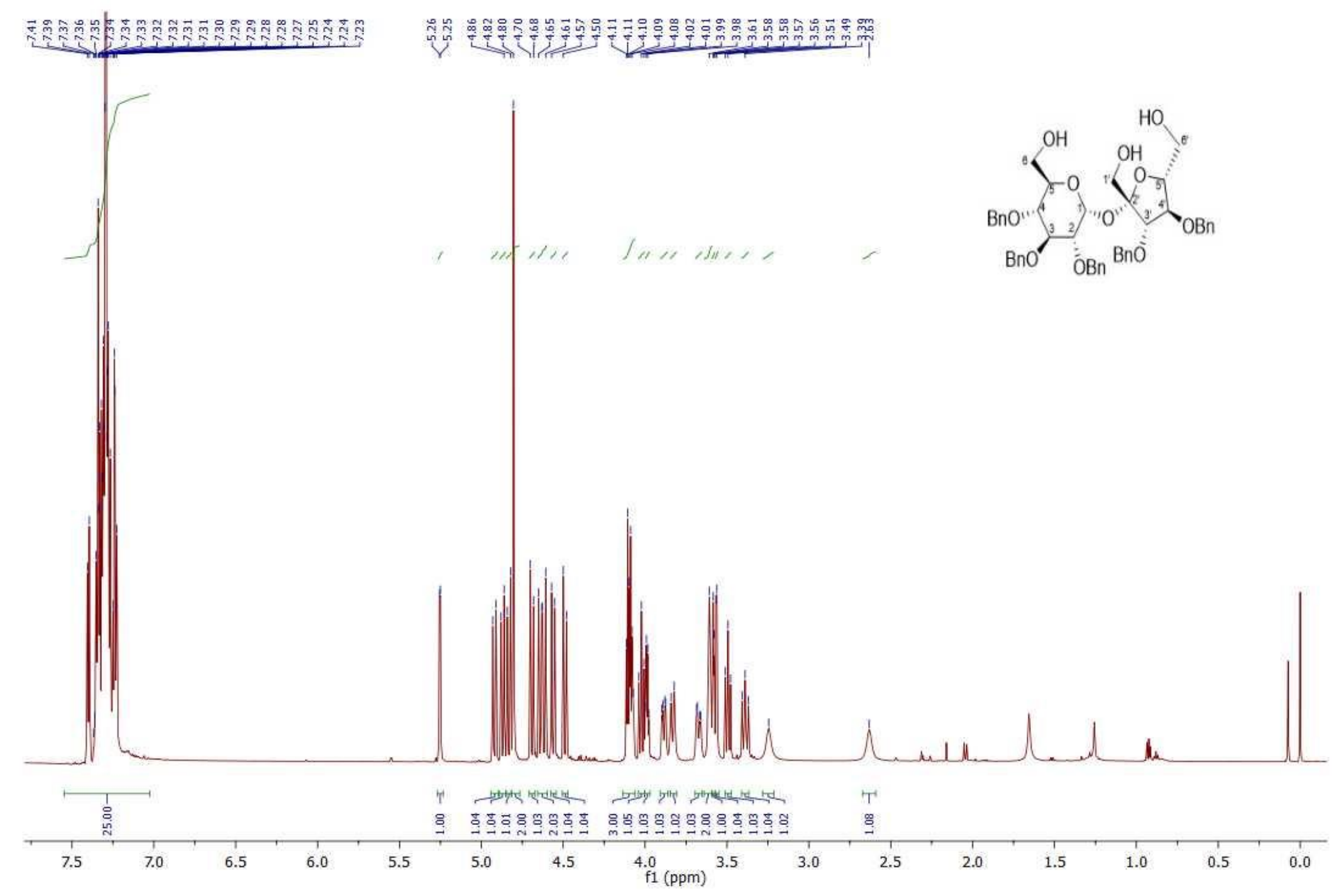

Figure S21. ${ }^{1} \mathrm{H}$ NMR spectrum of compound 3. 


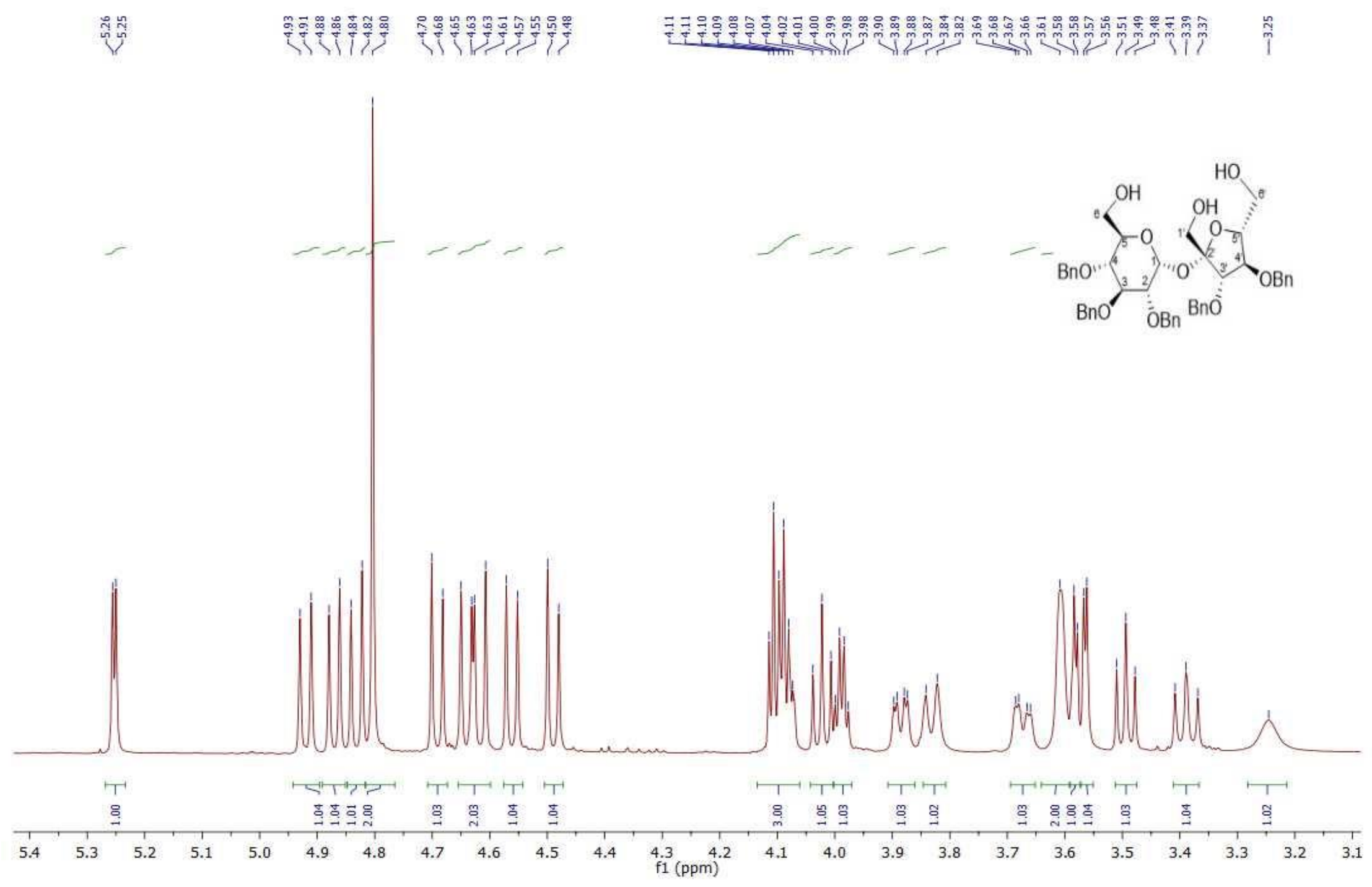

Figure S22. ${ }^{1} \mathrm{H}$ NMR spectrum of compound 3 (aliphatic part). 


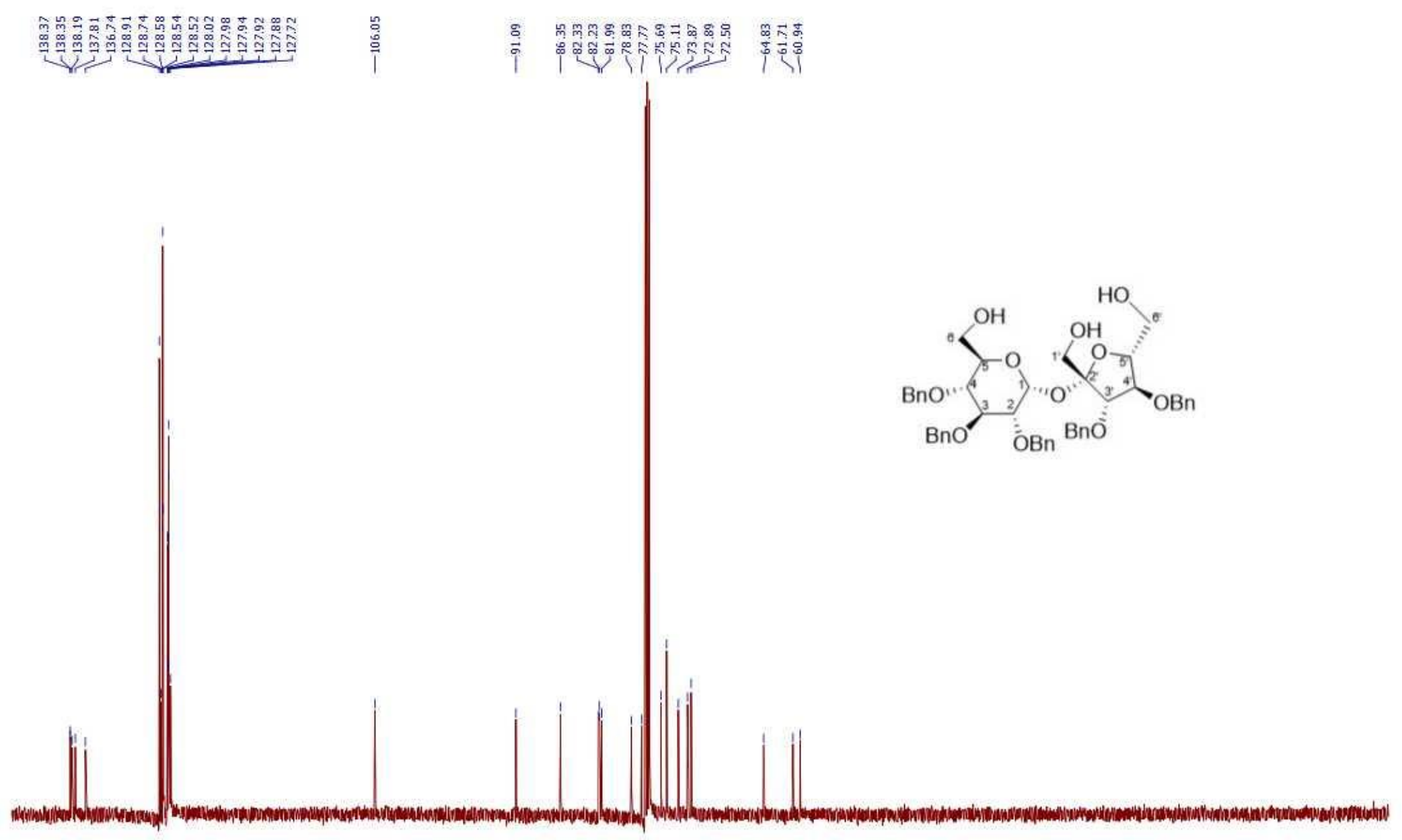

$\begin{array}{lllllllllllllllllllllllllllllllllll}140 & 135 & 130 & 125 & 120 & 115 & 110 & 105 & 100 & 95 & 90 & 85 & 80 & 75 & 70 & 65 & 60 & 55 & 50 & 45 & 40 & 35 & 30 & 25 & 20 & 15 & 10 & 5 & 0\end{array}$

Figure S23. ${ }^{13} \mathrm{C}$ NMR spectrum of compound 3. 


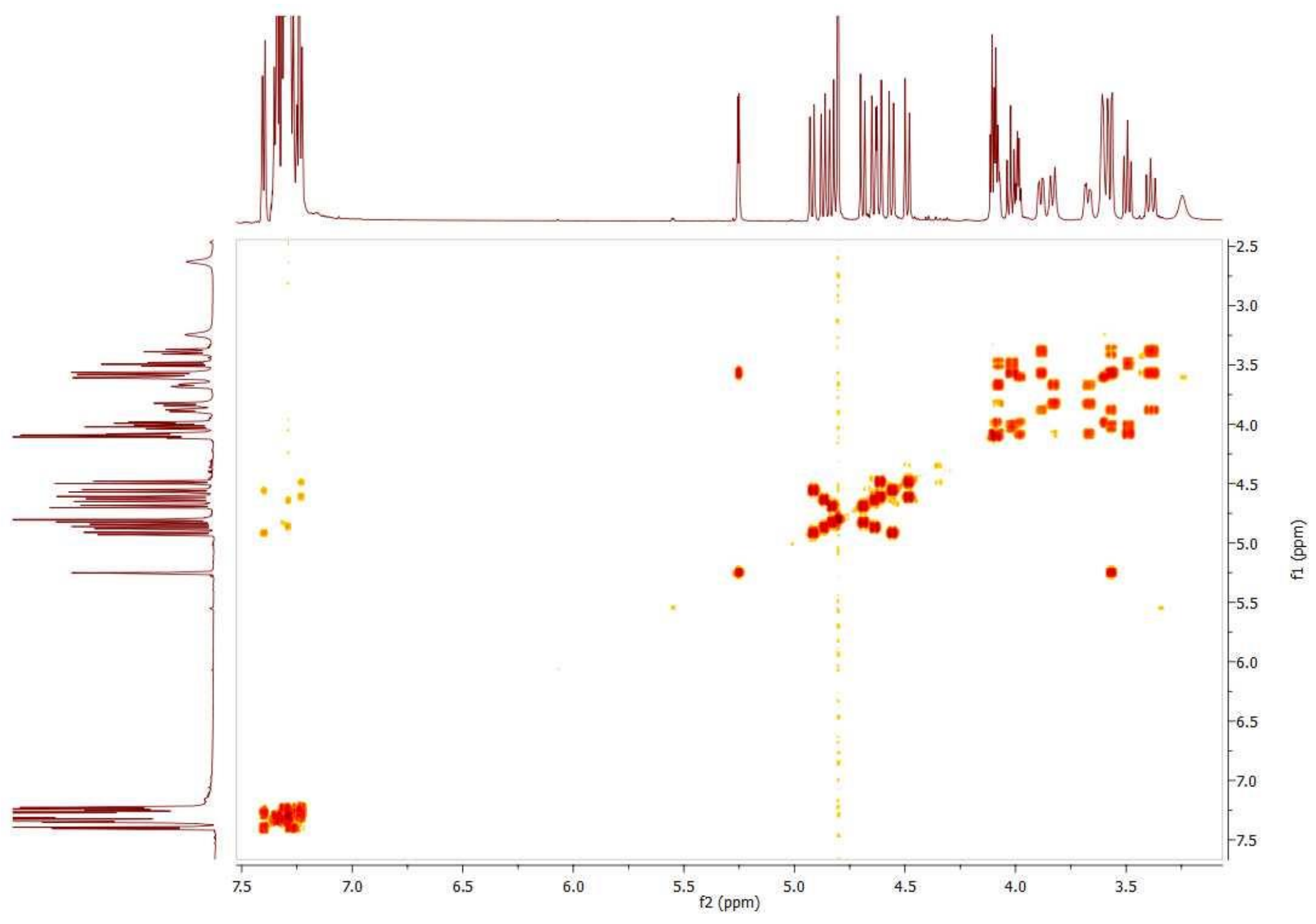

Figure S24. ${ }^{1} \mathrm{H}-{ }^{1} \mathrm{H}$ COSY spectrum of compound 3 . 


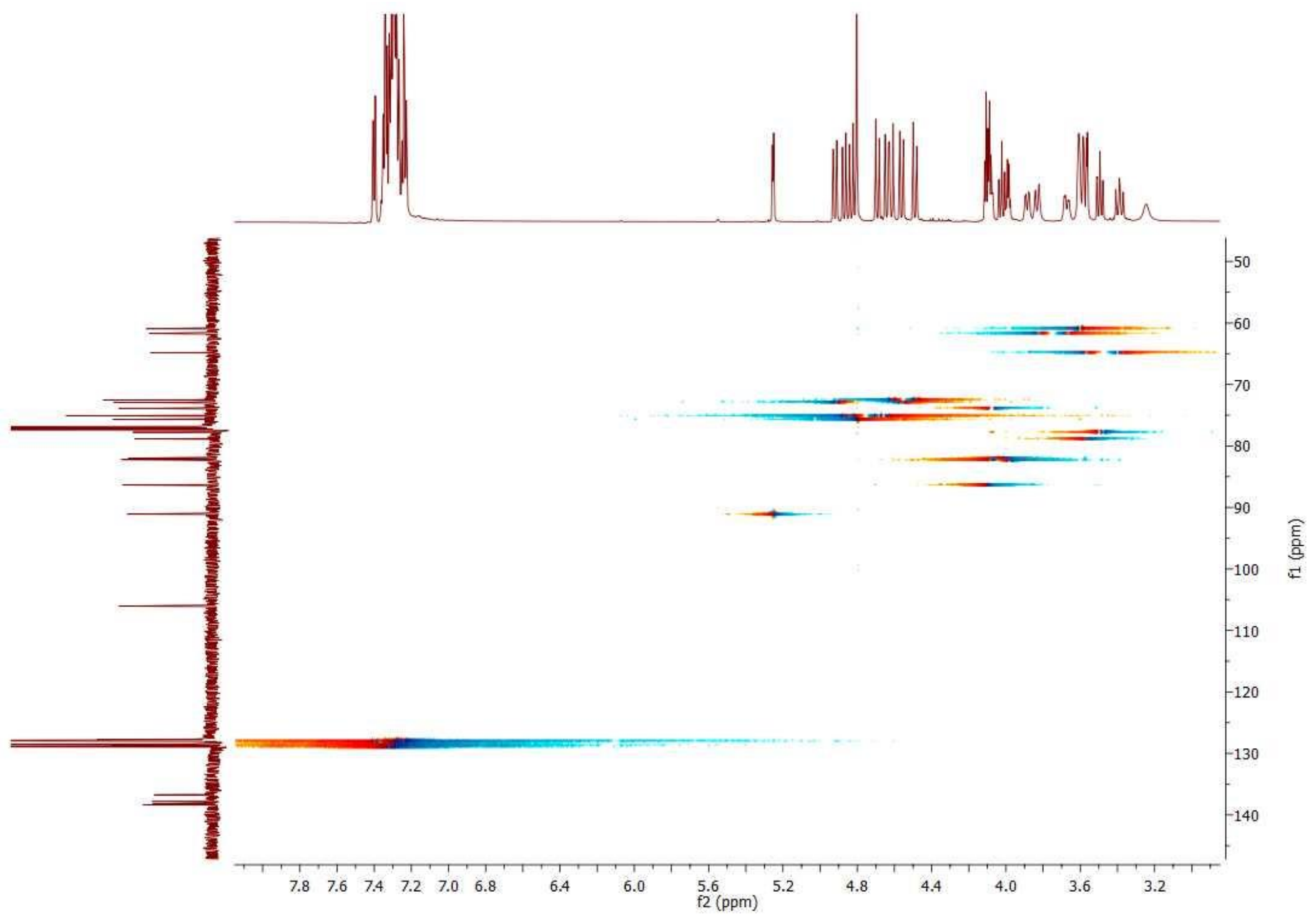

Figure S25. ${ }^{1} \mathrm{H}-{ }^{13} \mathrm{C}$ HSQC spectrum of compound 3 . 


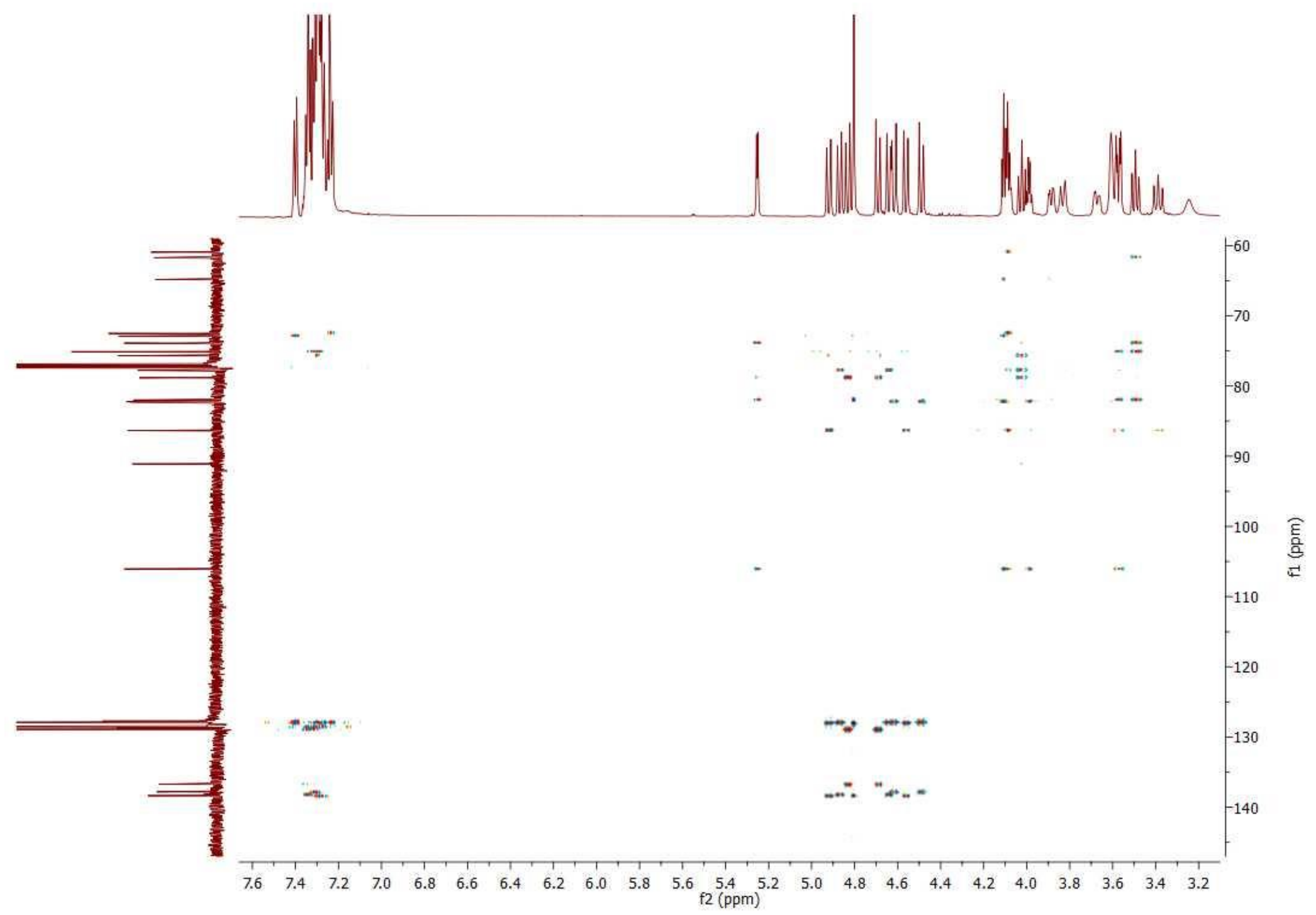

Figure S26. ${ }^{1} \mathrm{H}-{ }^{13} \mathrm{C}$ HMBC spectrum of compound 3 . 


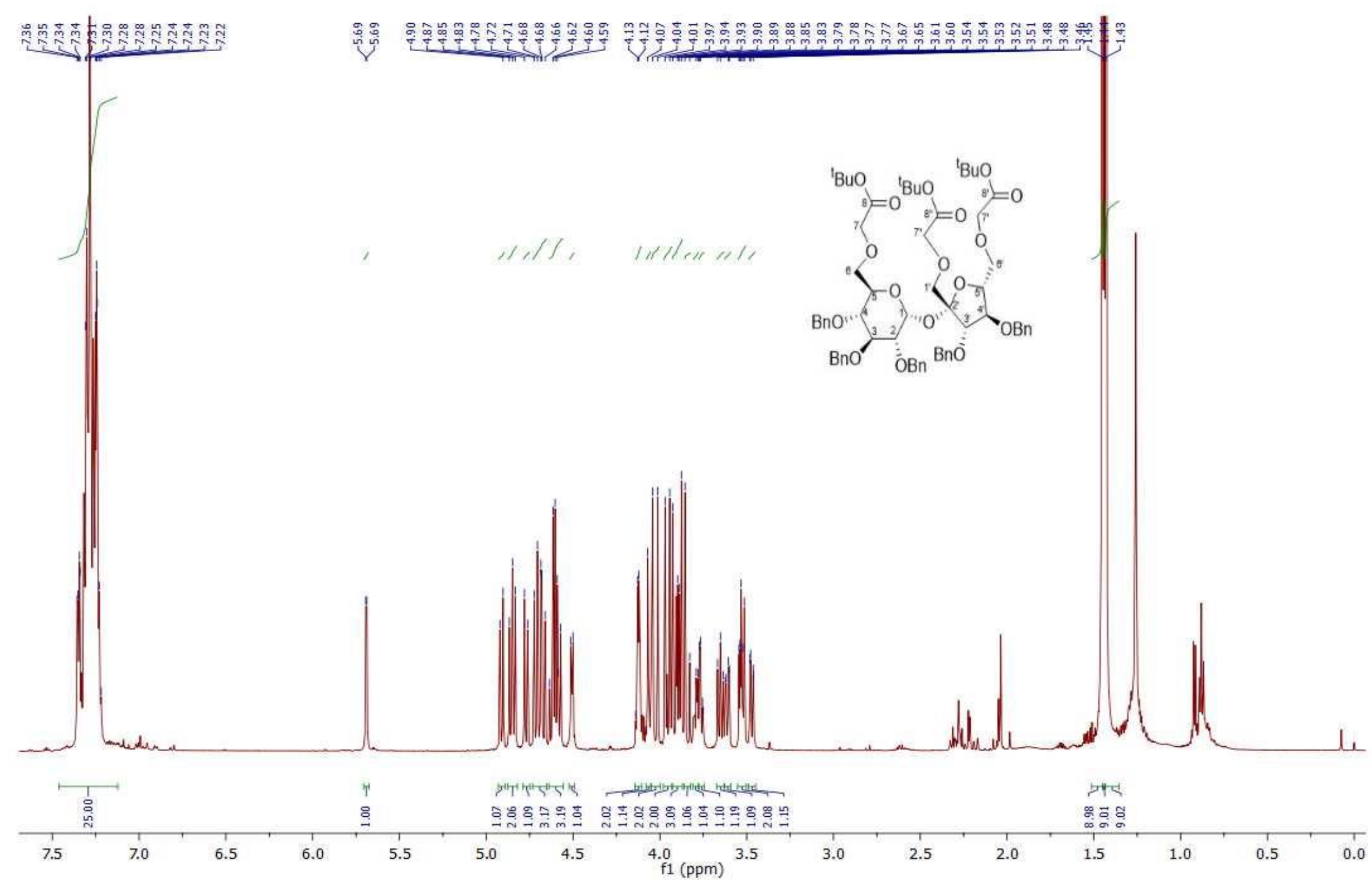

Figure S27. ${ }^{1} \mathrm{H}$ NMR spectrum of compound 4. 

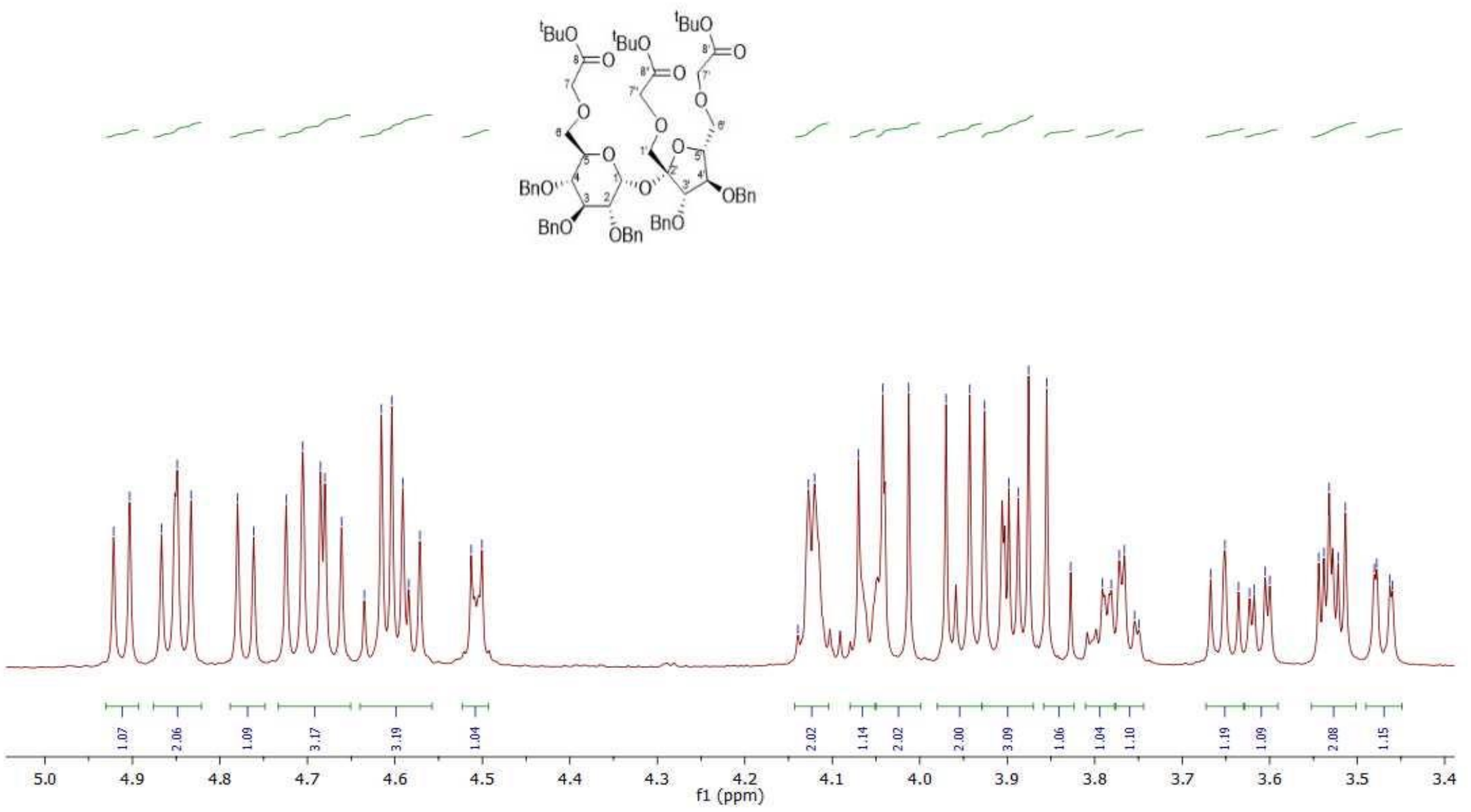

Figure S28. ${ }^{1} \mathrm{H}$ NMR spectrum of compound 4 (aliphatic part). 


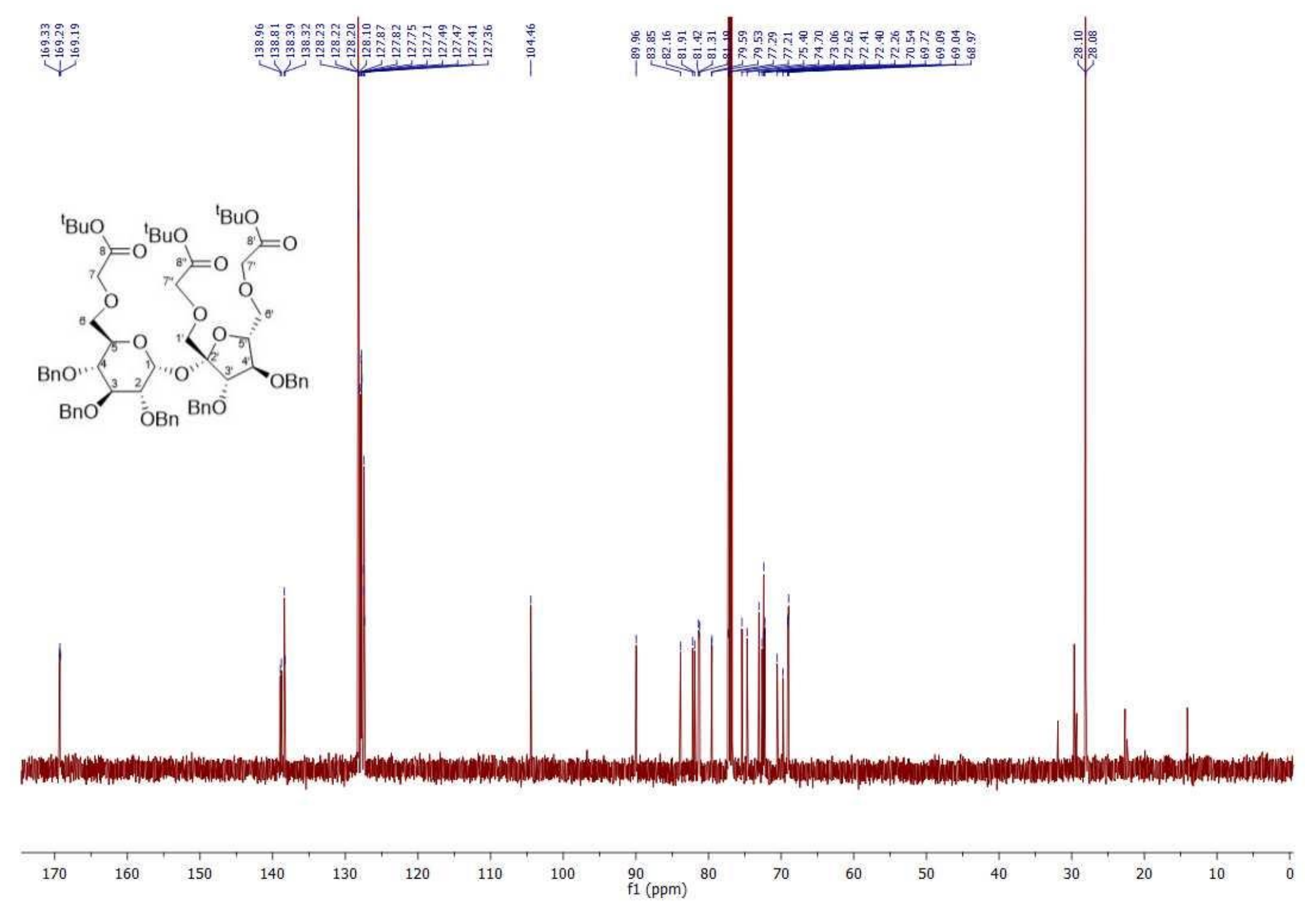

Figure S29. ${ }^{13} \mathrm{C}$ NMR spectrum of compound 4. 


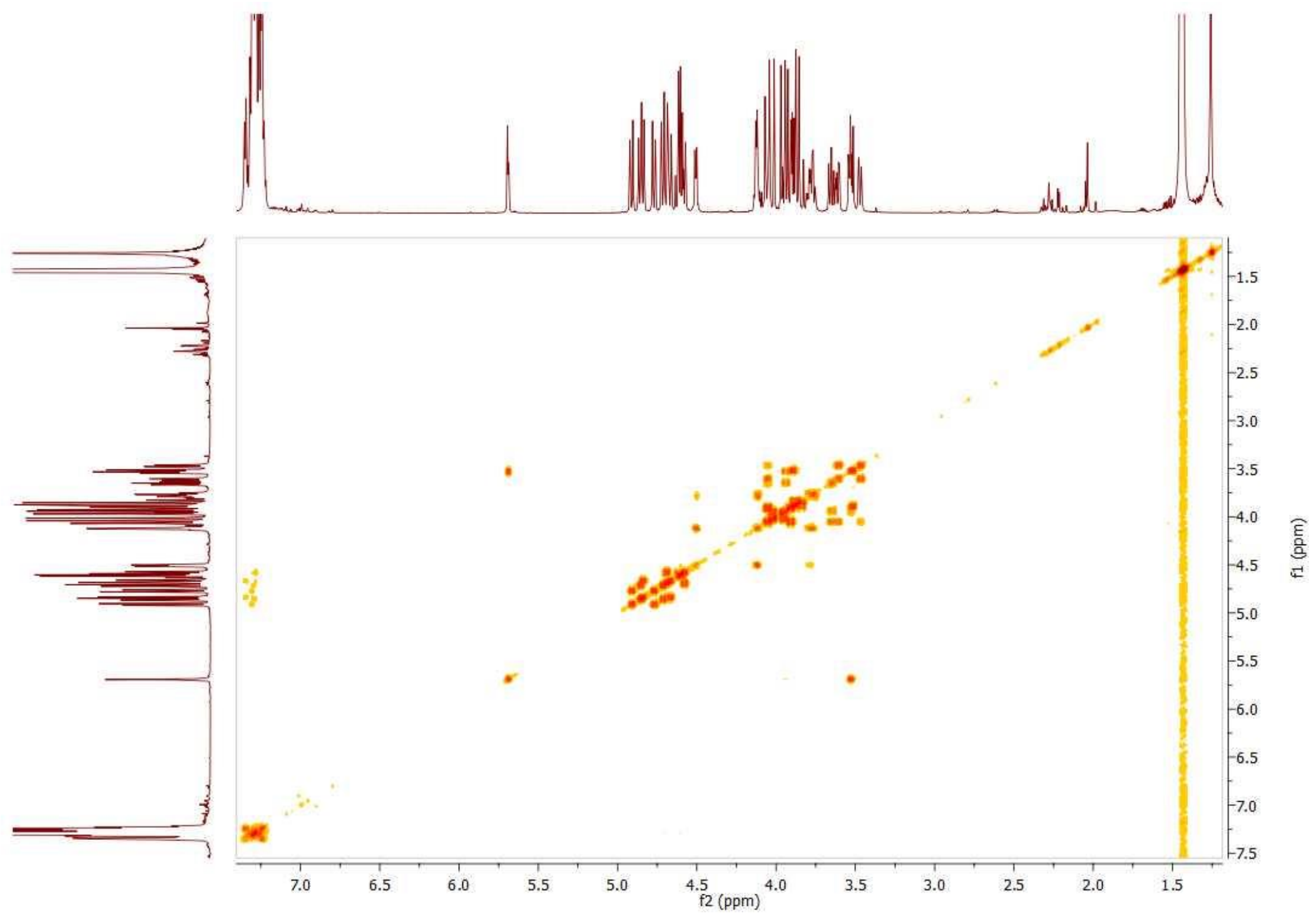

Figure S30. ${ }^{1} \mathrm{H}-{ }^{1} \mathrm{H}$ COSY spectrum of compound 4. 


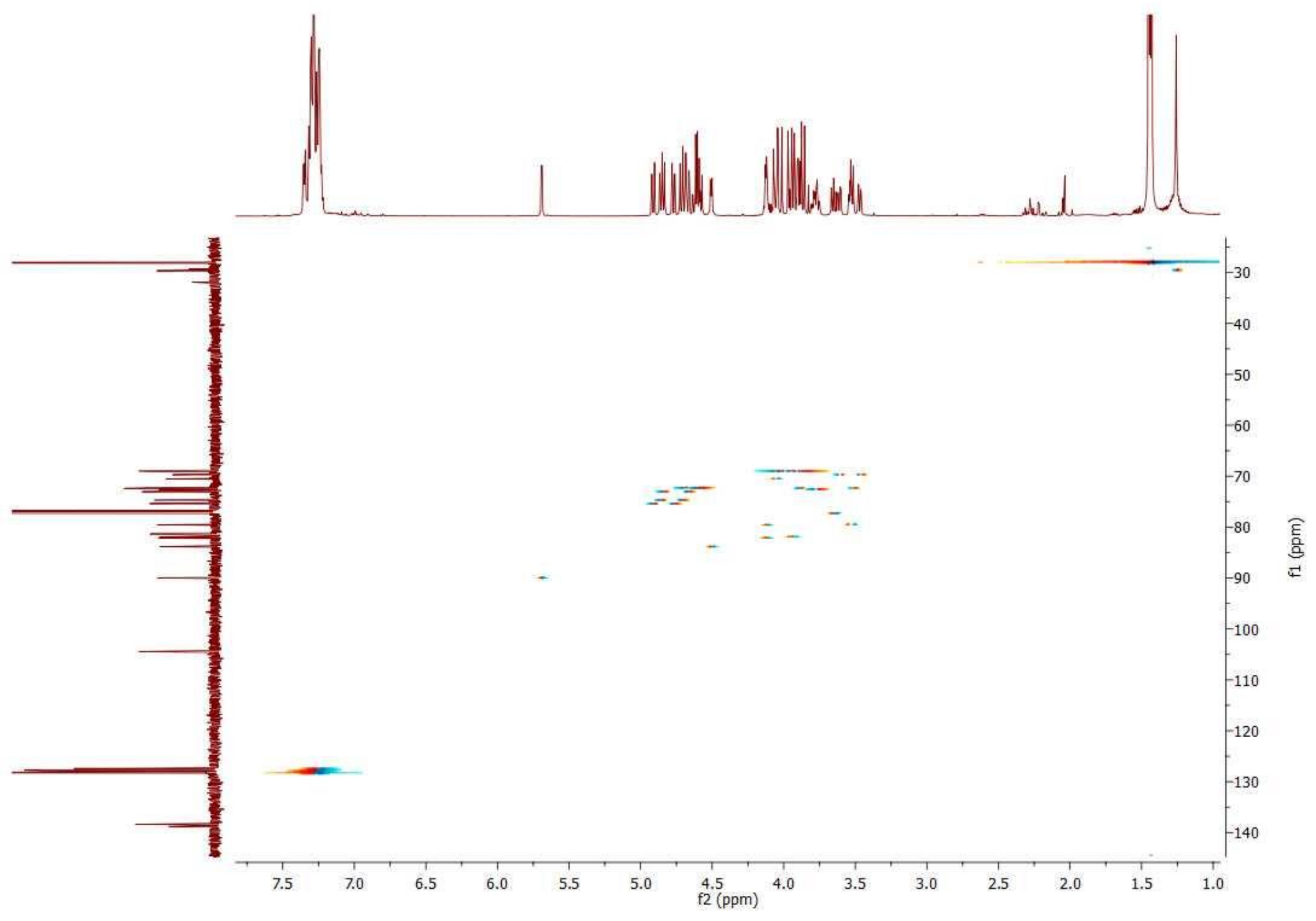

Figure S31. ${ }^{1} \mathrm{H}-{ }^{13} \mathrm{C}$ HSQC spectrum of compound 4. 


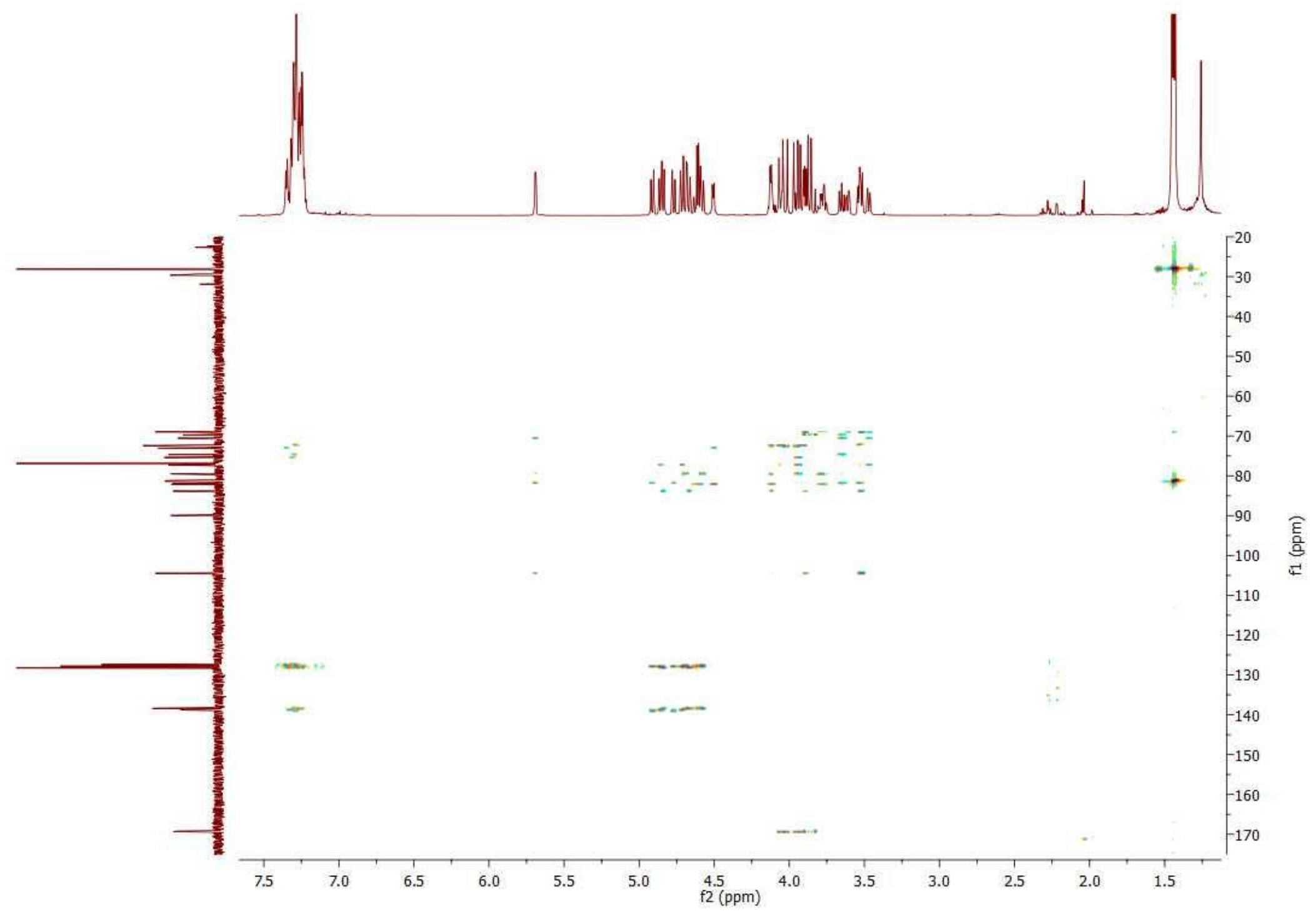

Figure S32. ${ }^{1} \mathrm{H}-{ }^{13} \mathrm{C}$ HMBC spectrum of compound 4. 

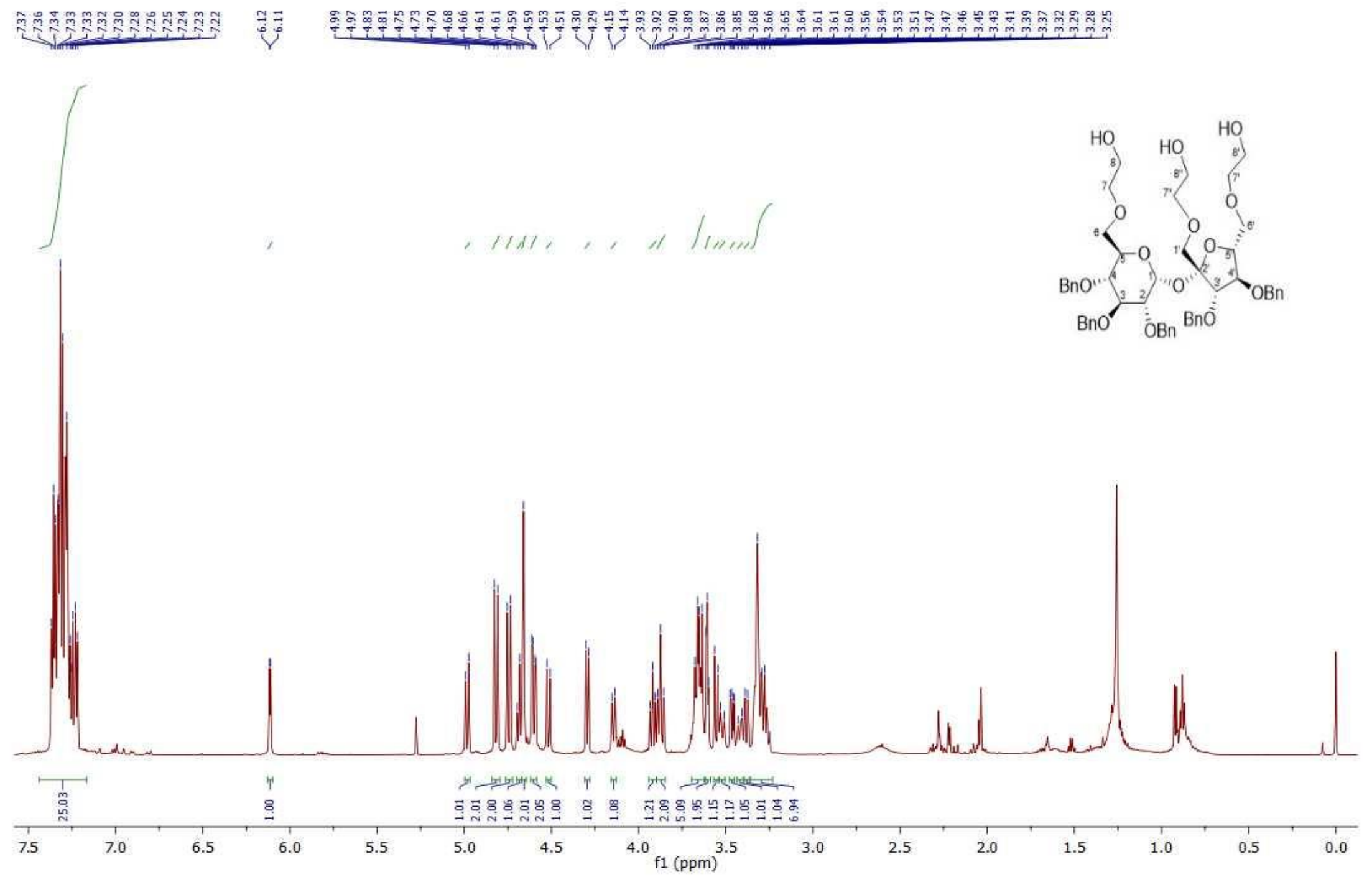

Figure S33. ${ }^{1} \mathrm{H}$ NMR spectrum of compound $\mathbf{5}$. 

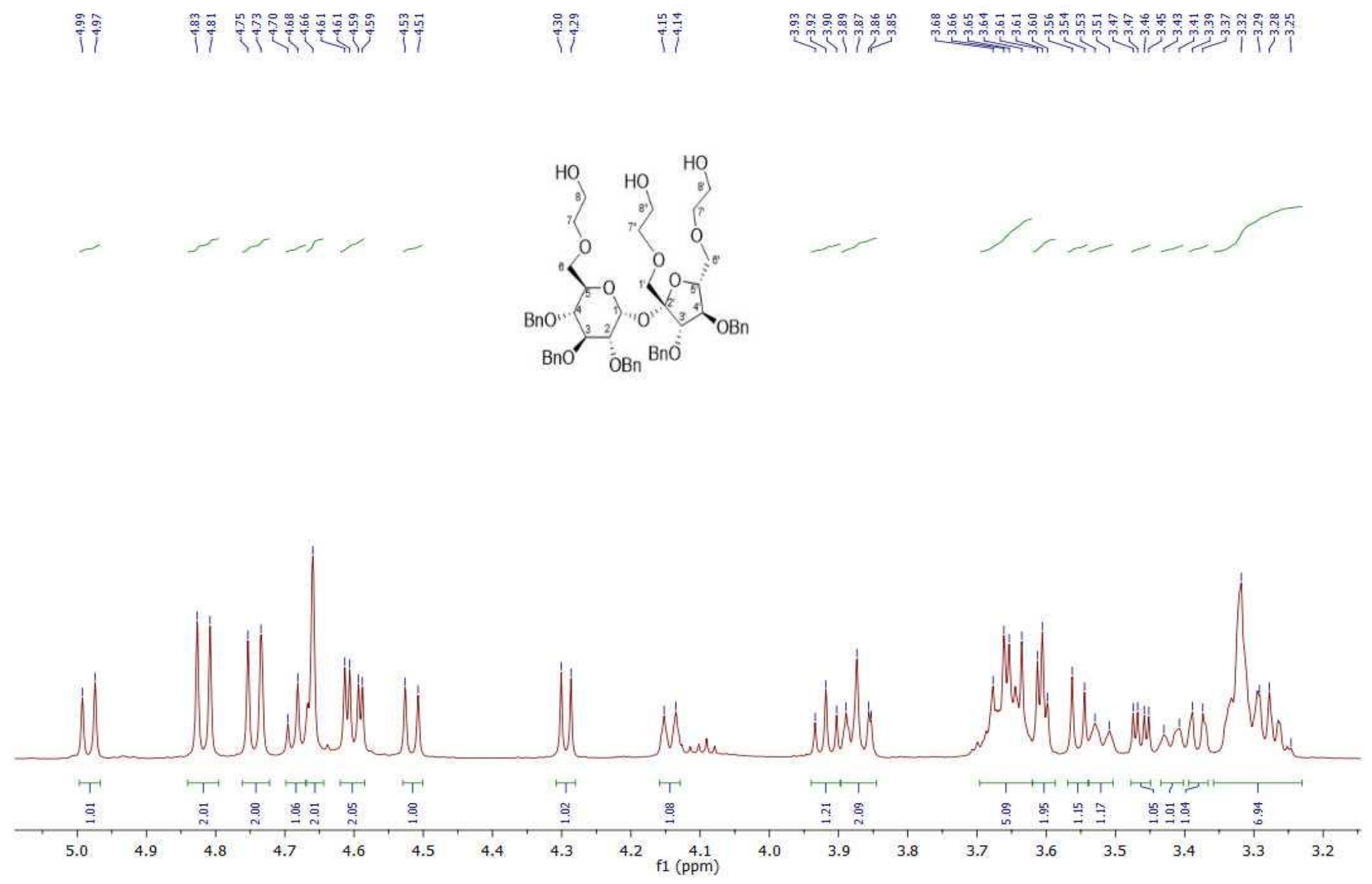

Figure S34. ${ }^{1} \mathrm{H}$ NMR spectrum of compound 5 (aliphatic part). 


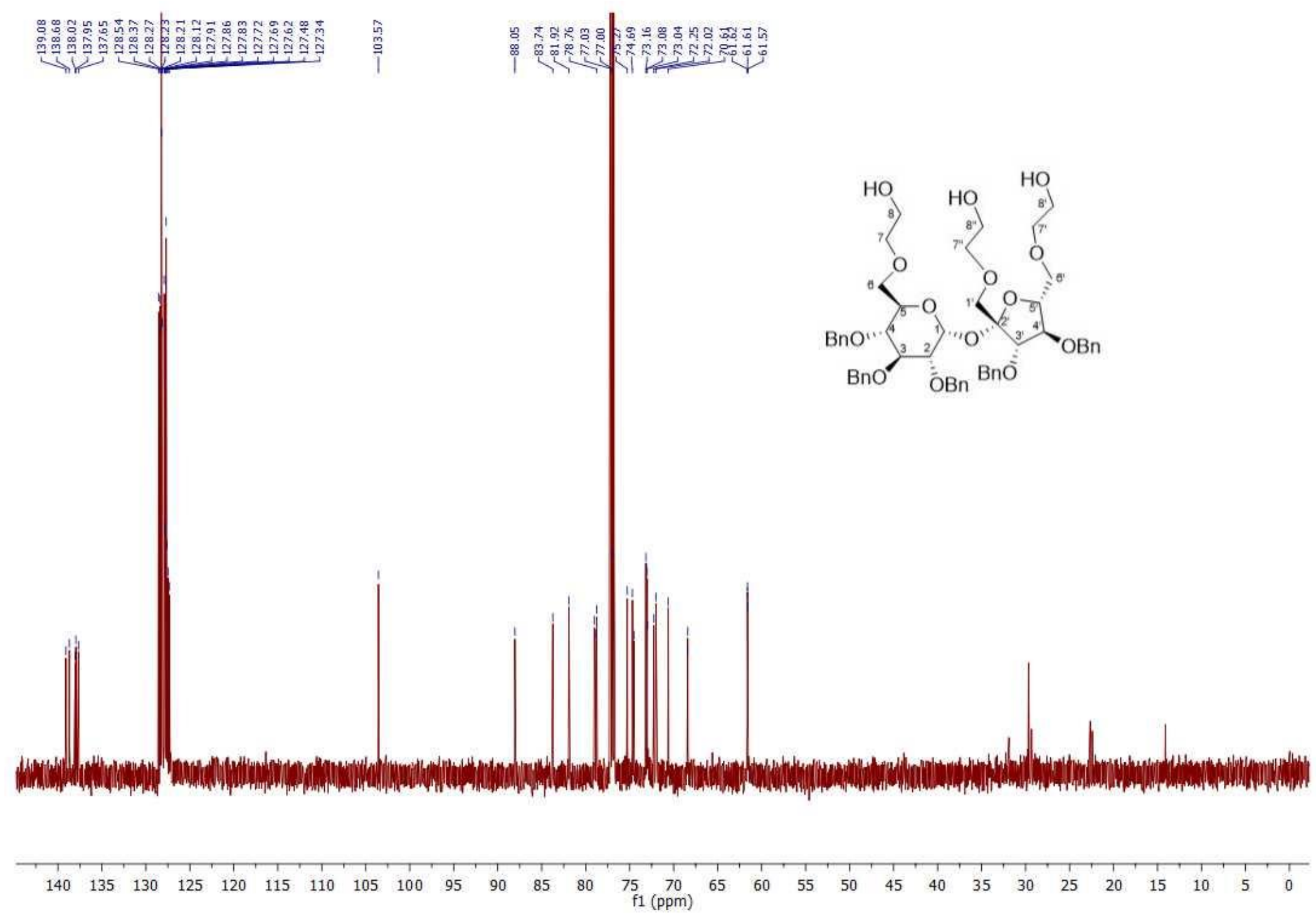

Figure S35. ${ }^{13} \mathrm{C}$ NMR spectrum of compound 5. 


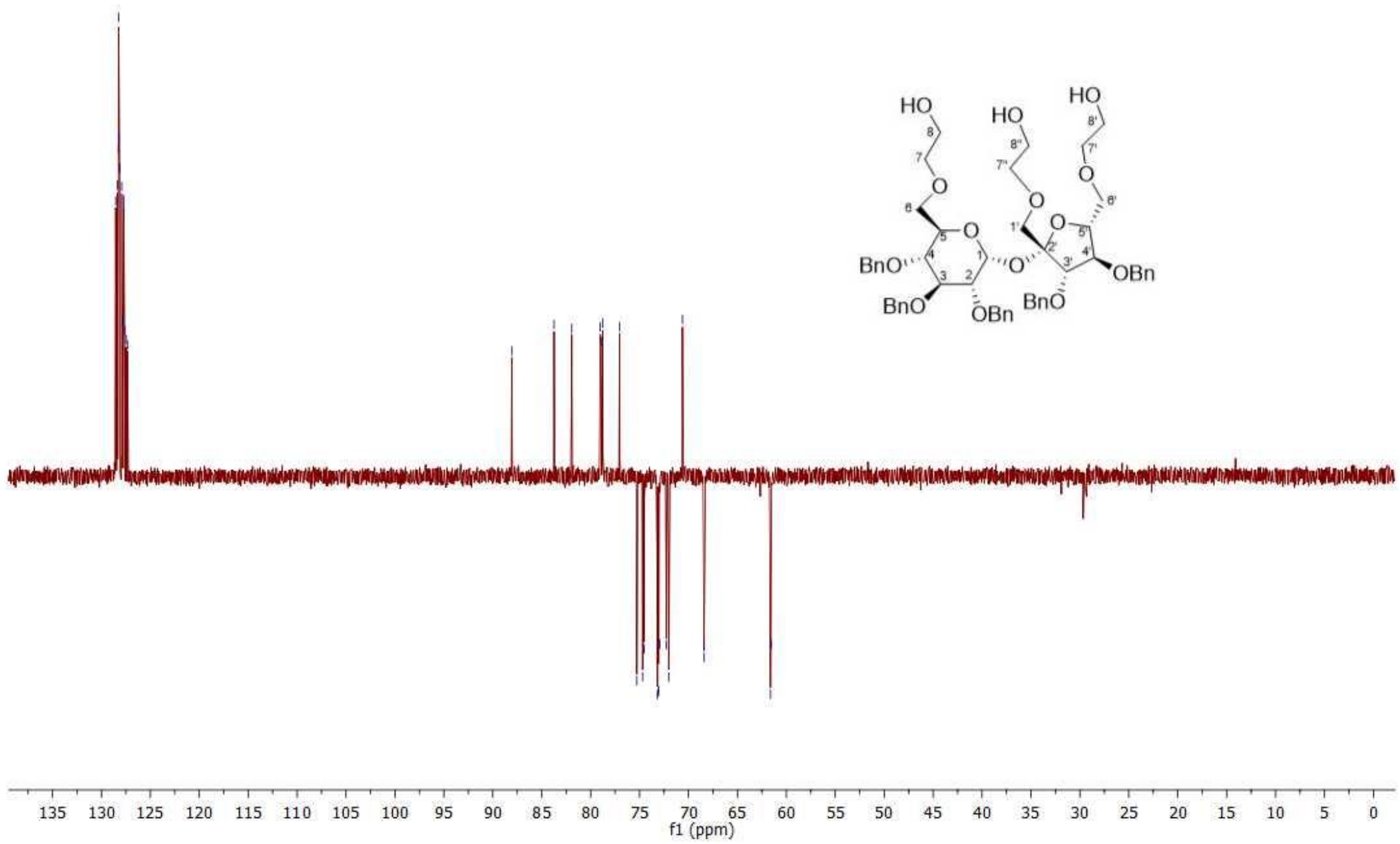

Figure S36. DEPT ${ }^{13} \mathrm{C}$ NMR spectrum of compound 5. 


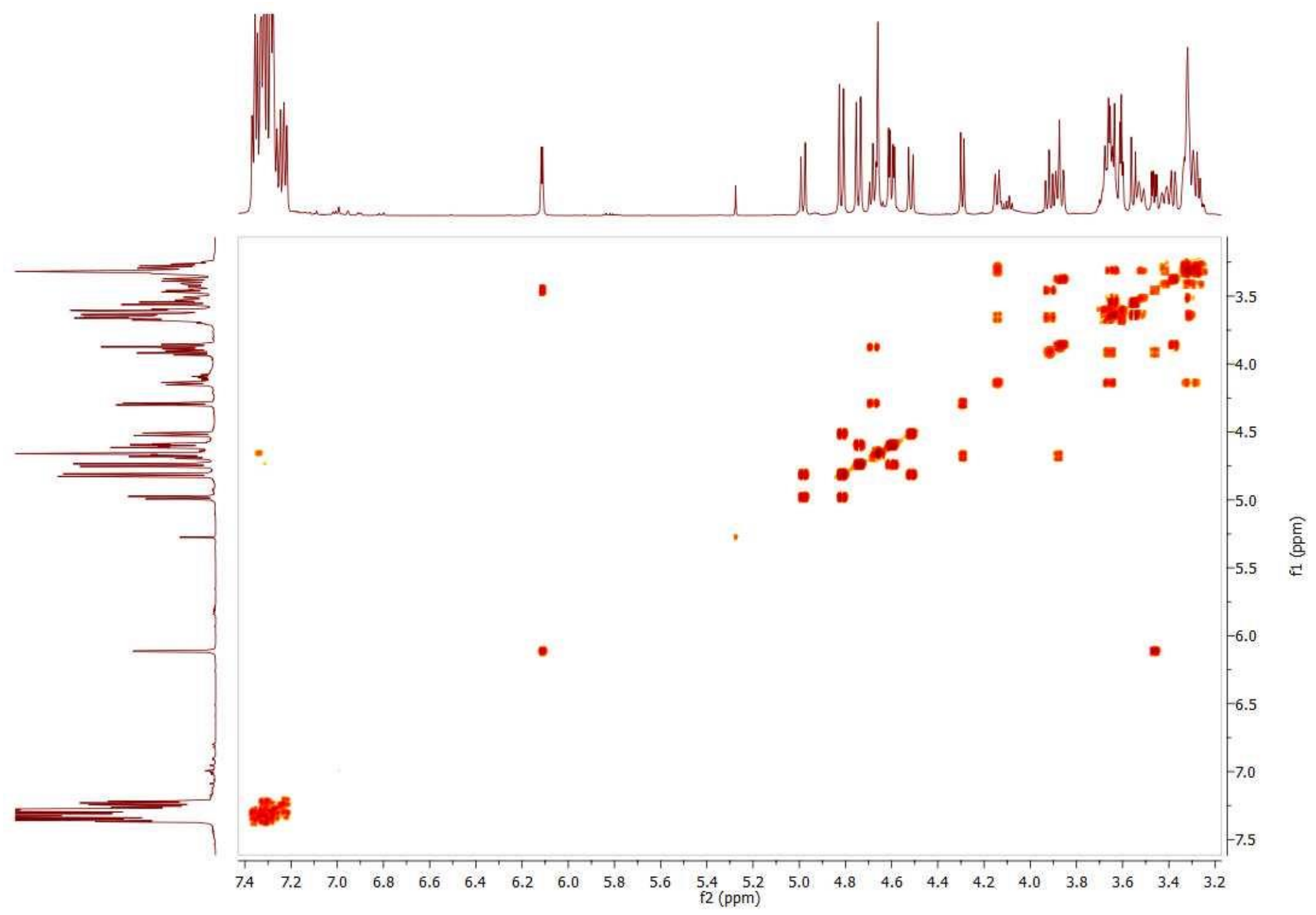

Figure S37. ${ }^{1} \mathrm{H}-{ }^{1} \mathrm{H}$ COSY spectrum of compound 5. 


$$
1
$$




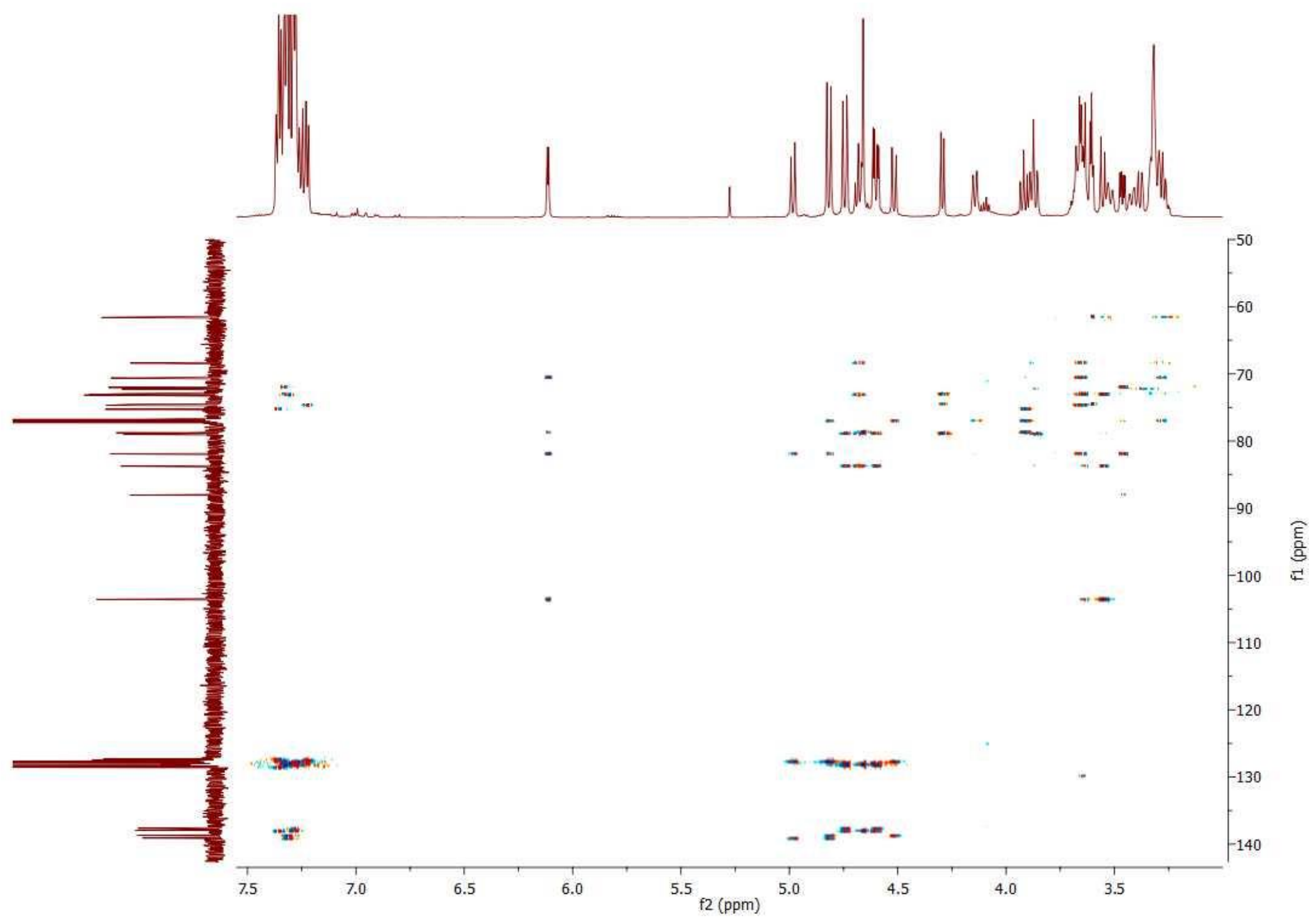

Figure S39. ${ }^{1} \mathrm{H}-{ }^{13} \mathrm{C}$ HMBC spectrum of compound 5. 


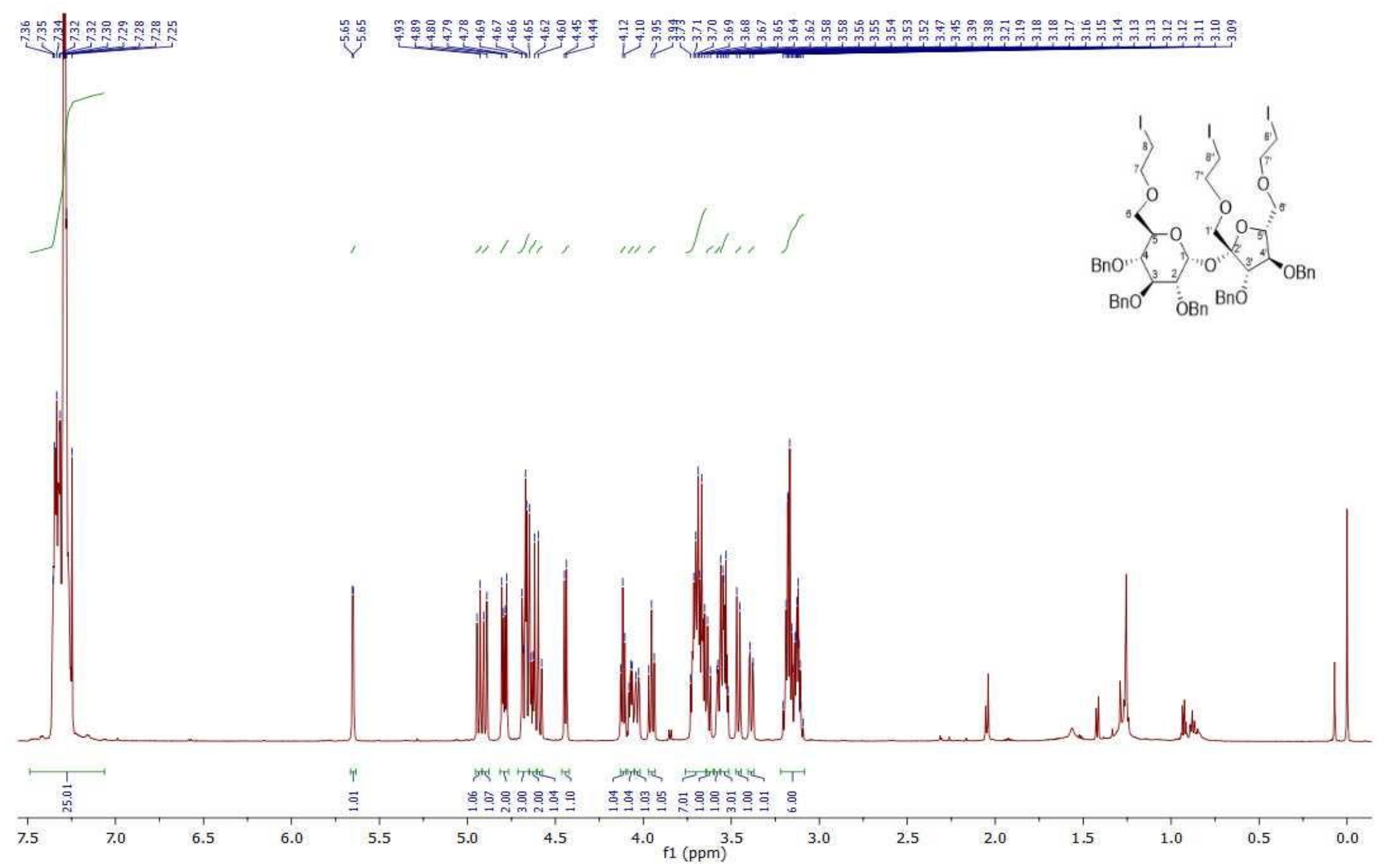

Figure S40. ${ }^{1} \mathrm{H}$ NMR spectrum of compound 6. 

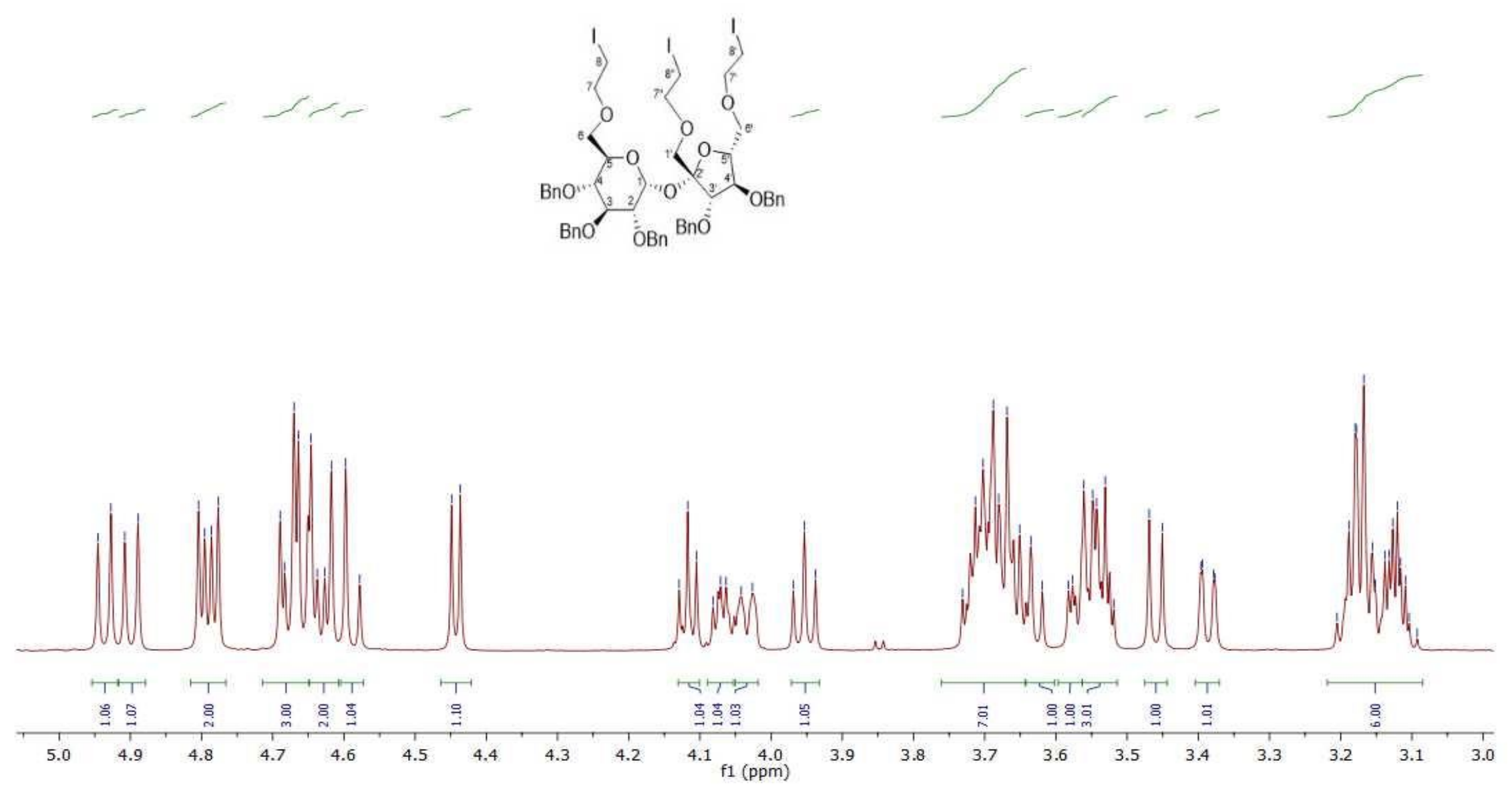

Figure S41. ${ }^{1} \mathrm{H}$ NMR spectrum of compound 6 (aliphatic part). 


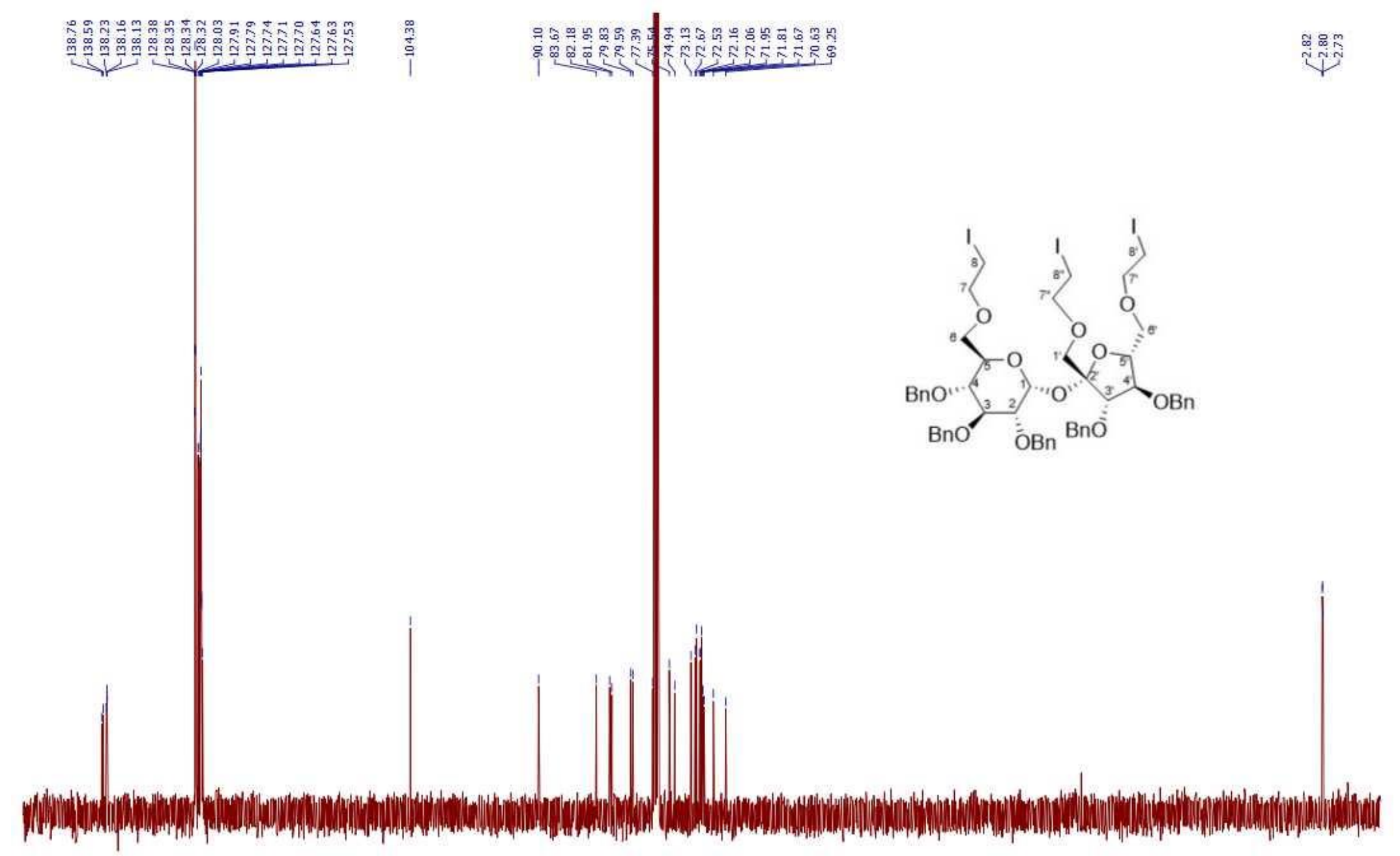

$\begin{array}{lllllllllllllllllllllllllllllllllllllllllllll}145 & 140 & 135 & 130 & 125 & 120 & 115 & 110 & 105 & 100 & 95 & 90 & 85 & 80 & 75 & 70 & 65 & 60 & 55 & 50 & 45 & 40 & 35 & 30 & 25 & 20 & 15 & 10 & 5 & 0\end{array}$

Figure S42. ${ }^{13} \mathrm{C}$ NMR spectrum of compound 6. 


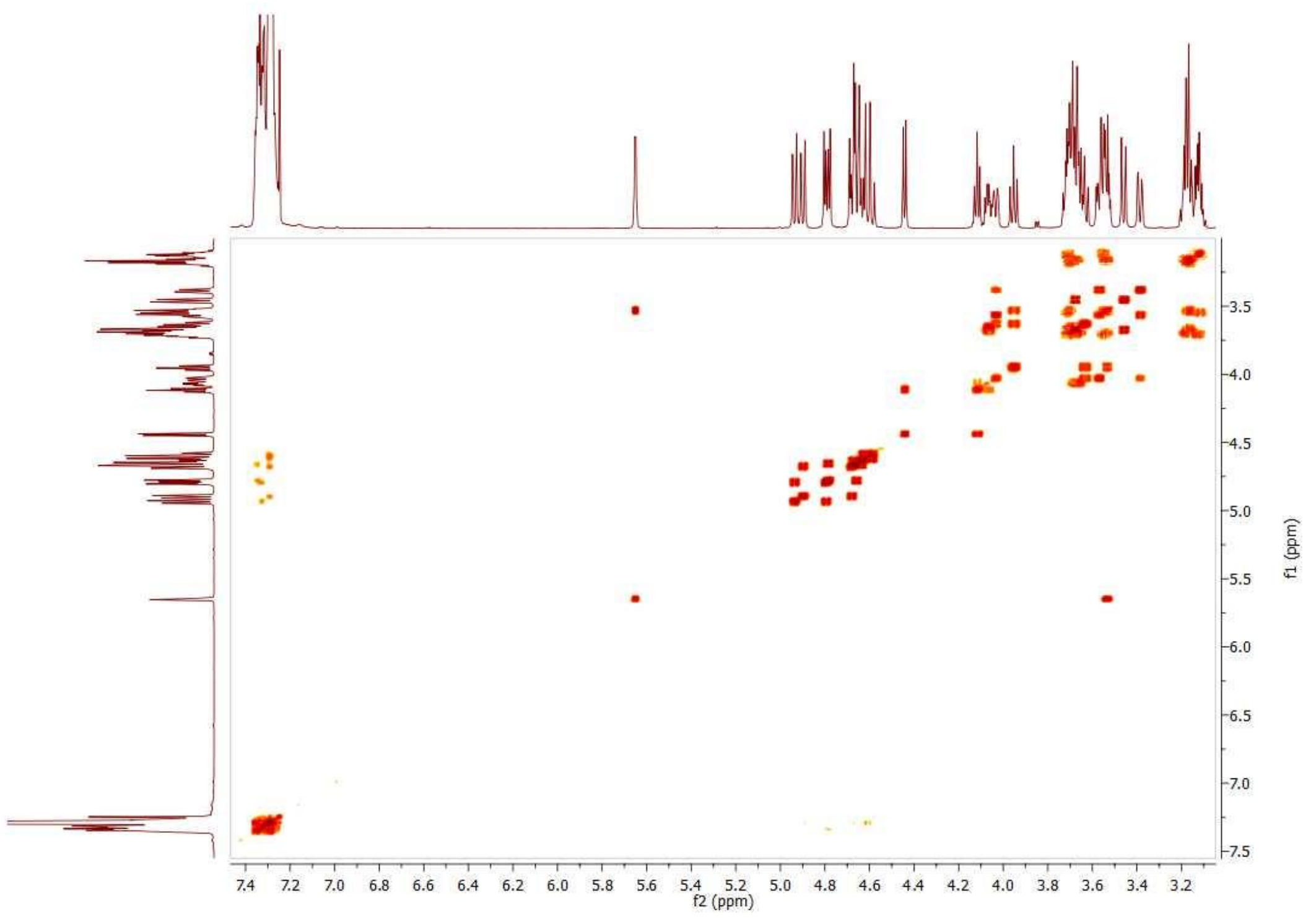

Figure S43. ${ }^{1} \mathrm{H}-{ }^{1} \mathrm{H}$ COSY spectrum of compound 6. 



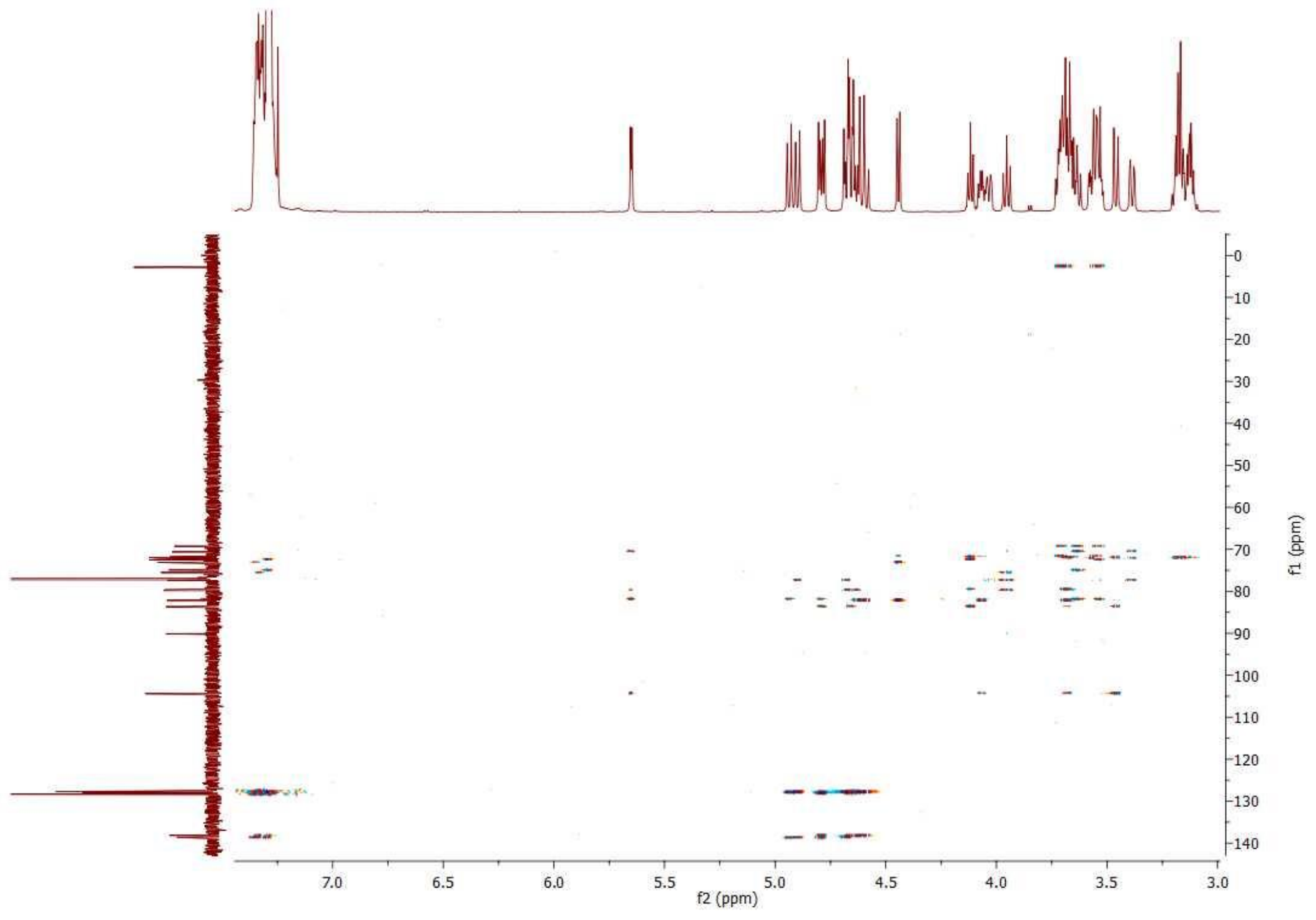

Figure S45. ${ }^{1} \mathrm{H}-{ }^{13} \mathrm{C}$ HMBC spectrum of compound 6. 


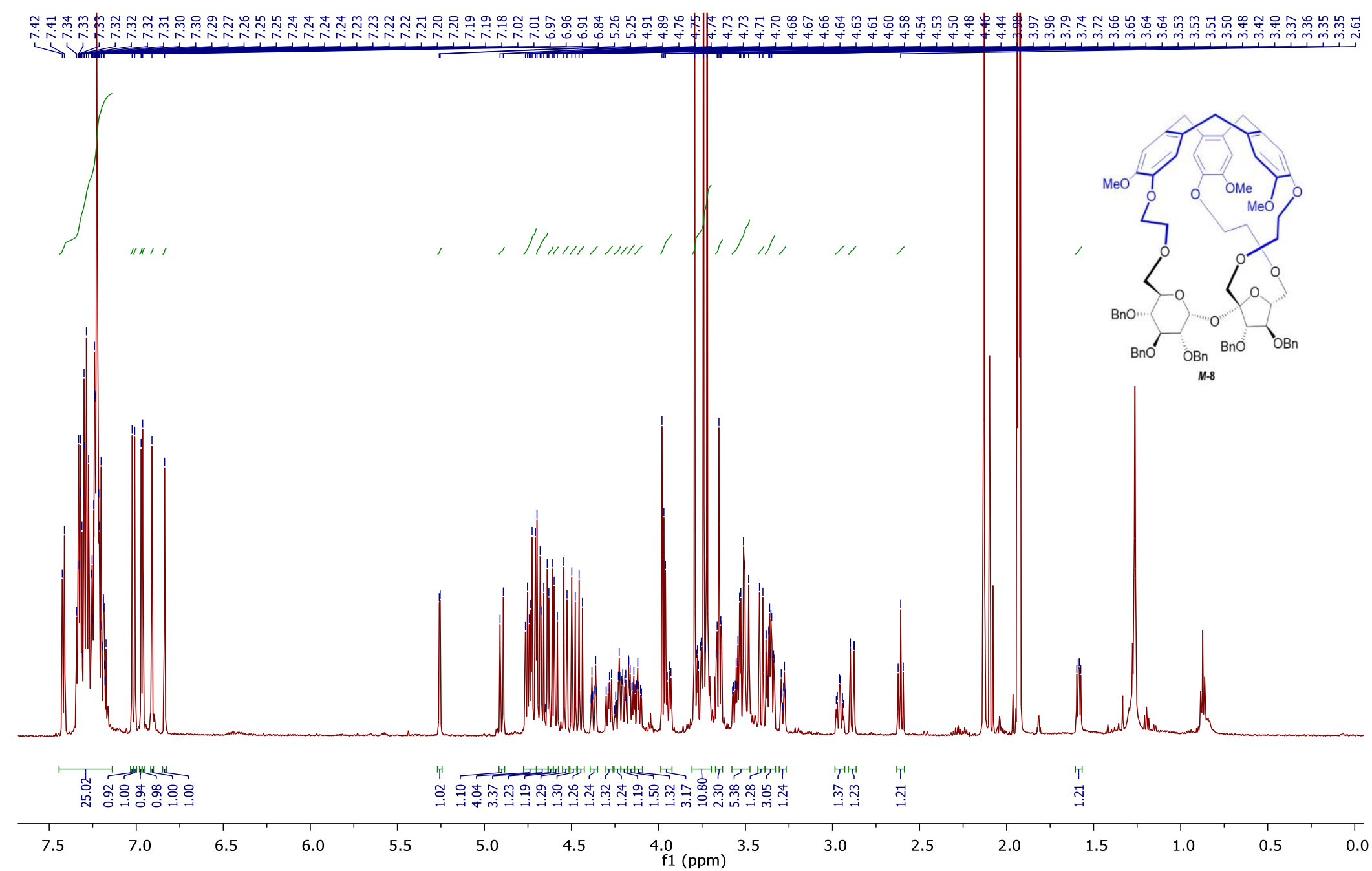

Figure S46. ${ }^{1} \mathrm{H}$ NMR spectrum of compound $\boldsymbol{M - 8}$. 


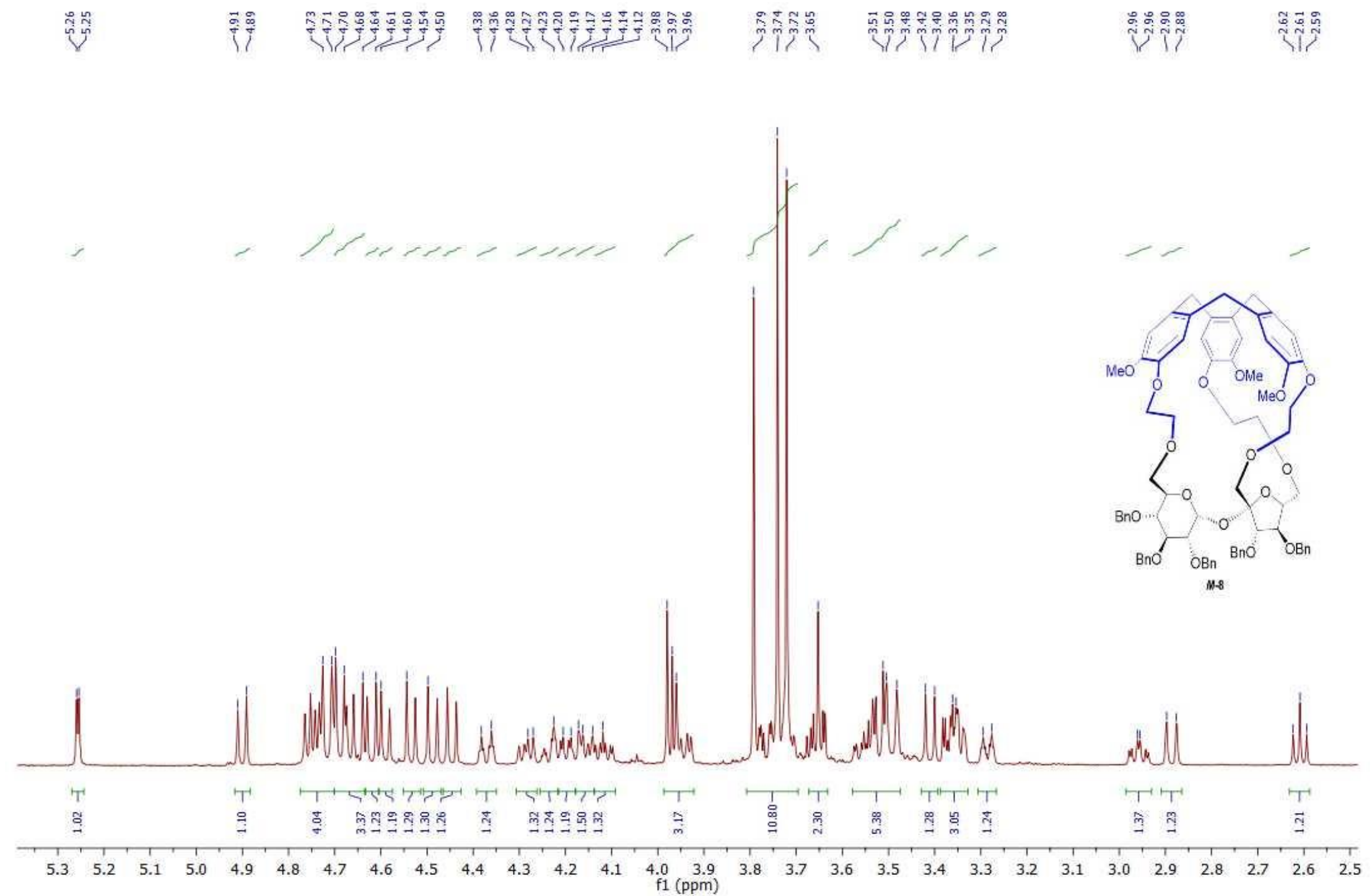

Figure S47. ${ }^{1} \mathrm{H}$ NMR spectrum of compound $\boldsymbol{M - 8}$ (aliphatic part). 


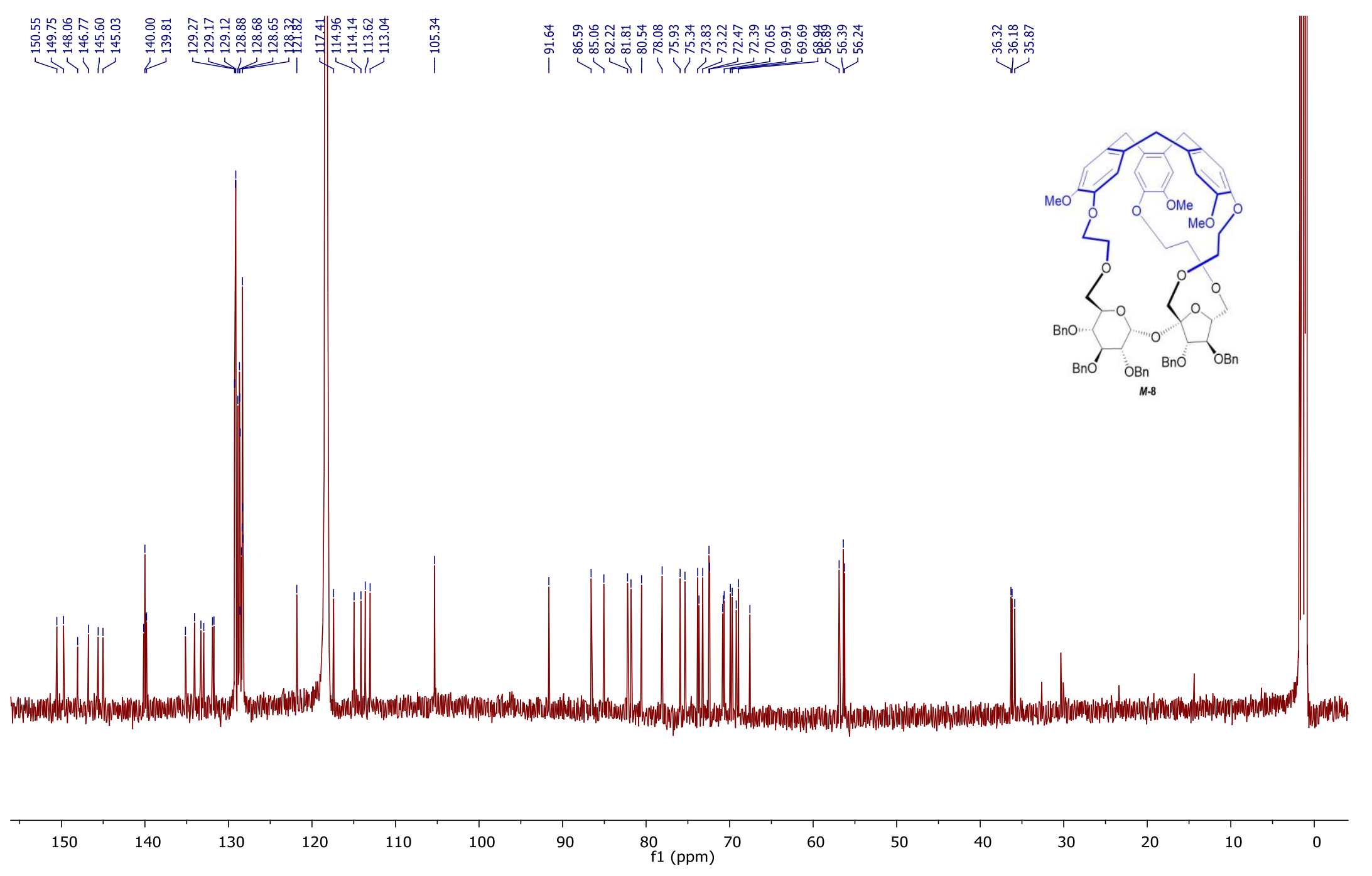

Figure S48. ${ }^{13} \mathrm{C}$ NMR spectrum of compound $\boldsymbol{M - 8}$. 

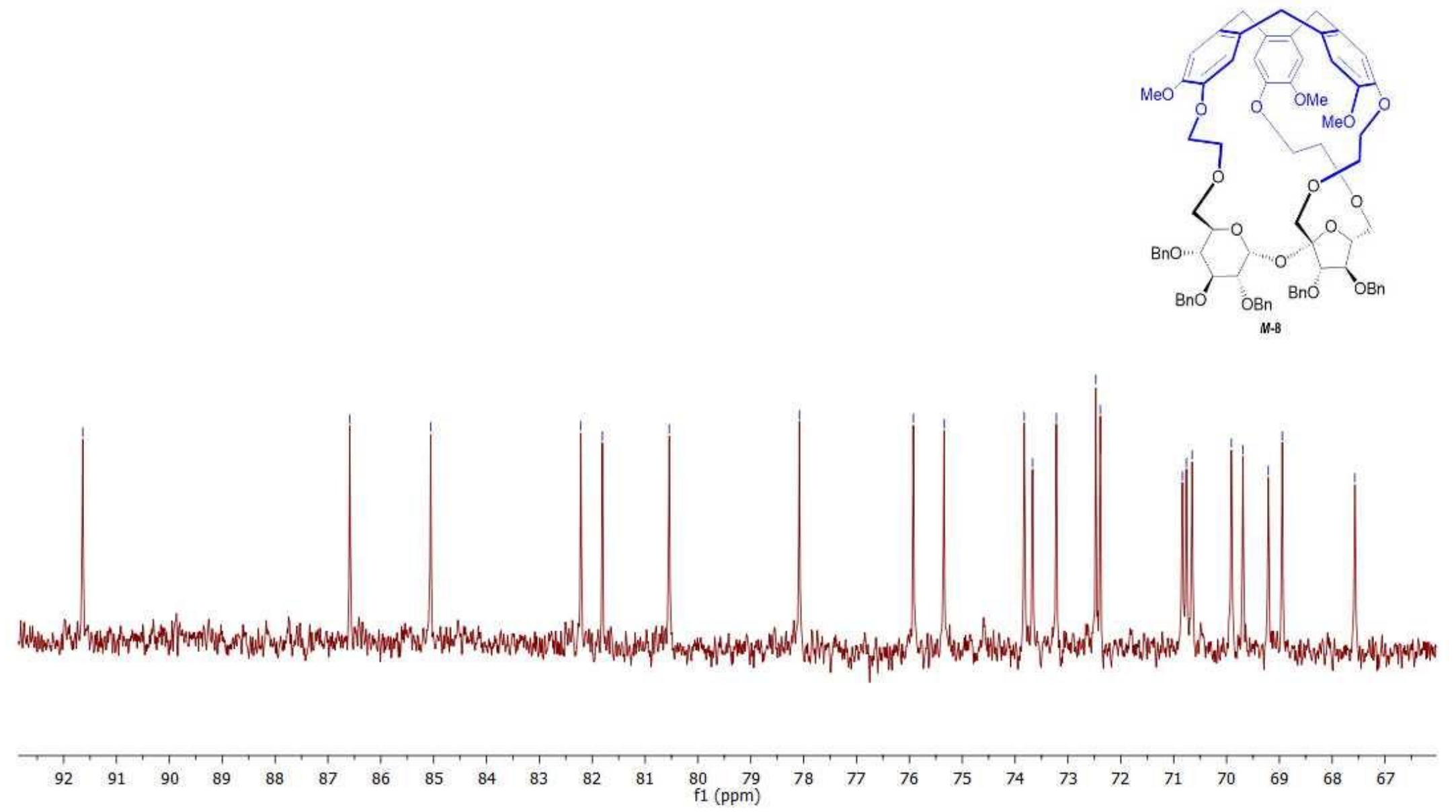

Figure S49. ${ }^{13} \mathrm{C}$ NMR spectrum of compound $\boldsymbol{M - 8}$ (aliphatic part). 


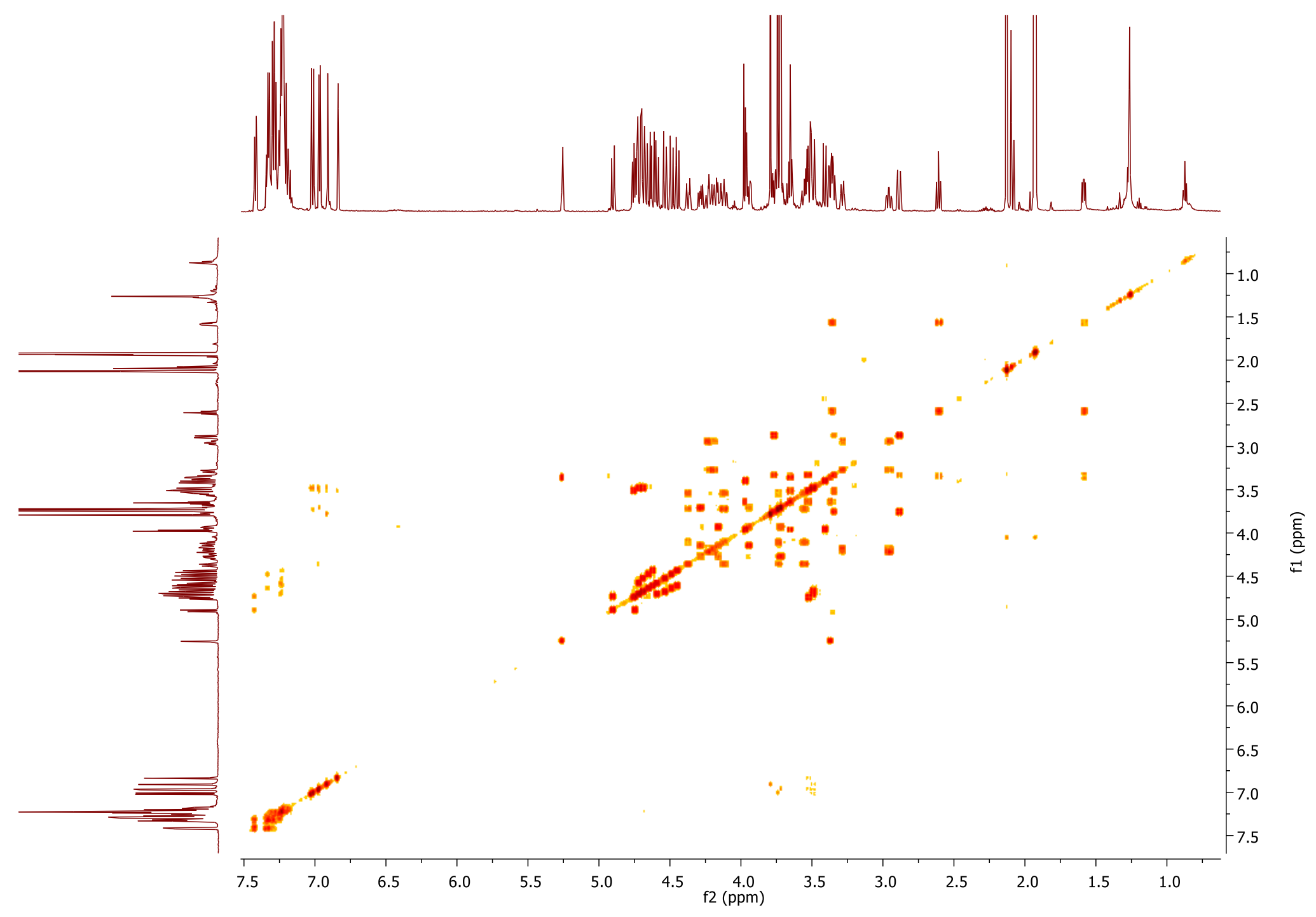

Figure S50. ${ }^{1} \mathrm{H}-{ }^{1} \mathrm{H}$ COSY spectrum of compound $\boldsymbol{M - 8}$. 

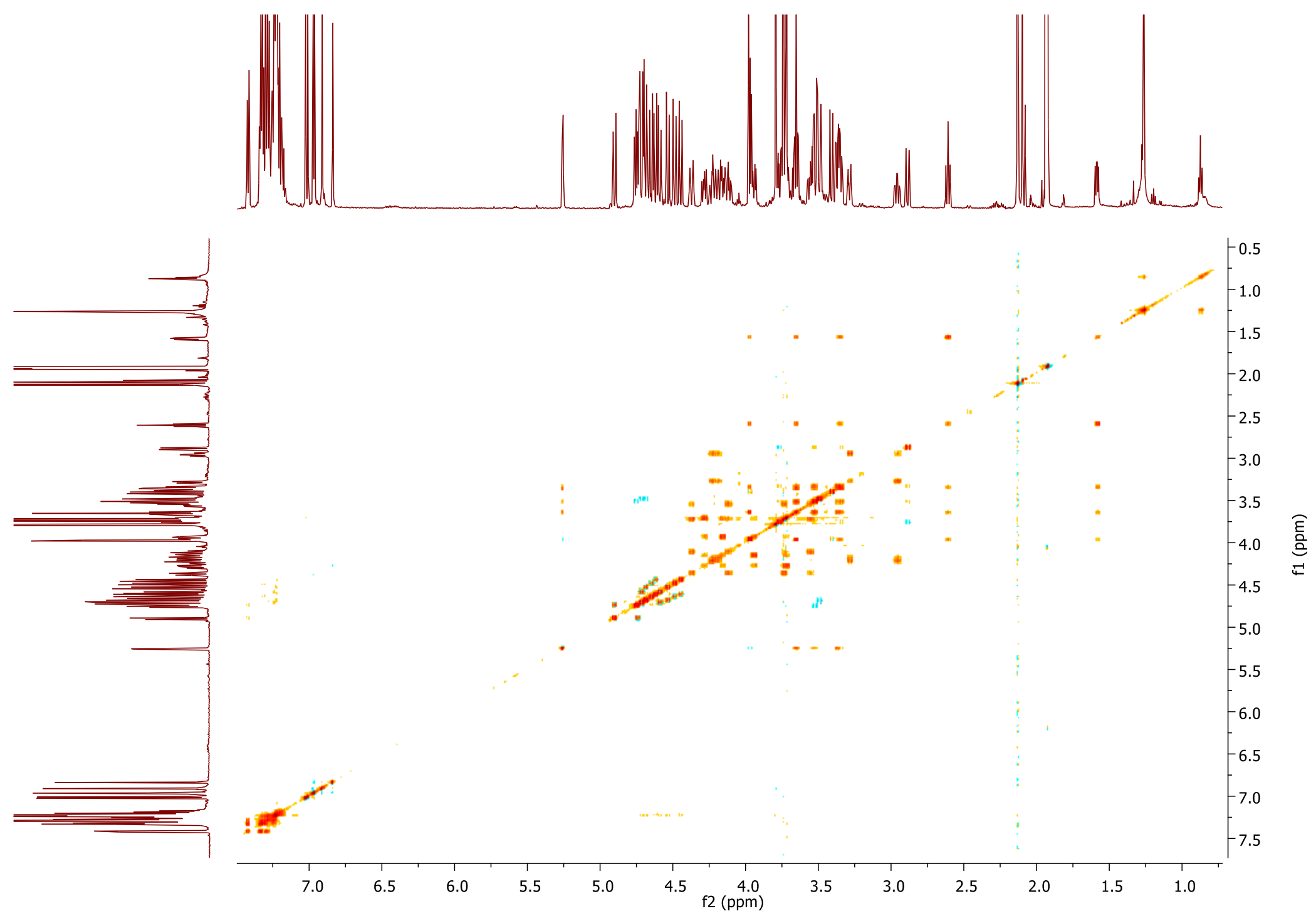

Figure S51. ${ }^{1} \mathrm{H}-{ }^{1} \mathrm{H}$ TOCSY spectrum of compound $\boldsymbol{M - 8}$. 


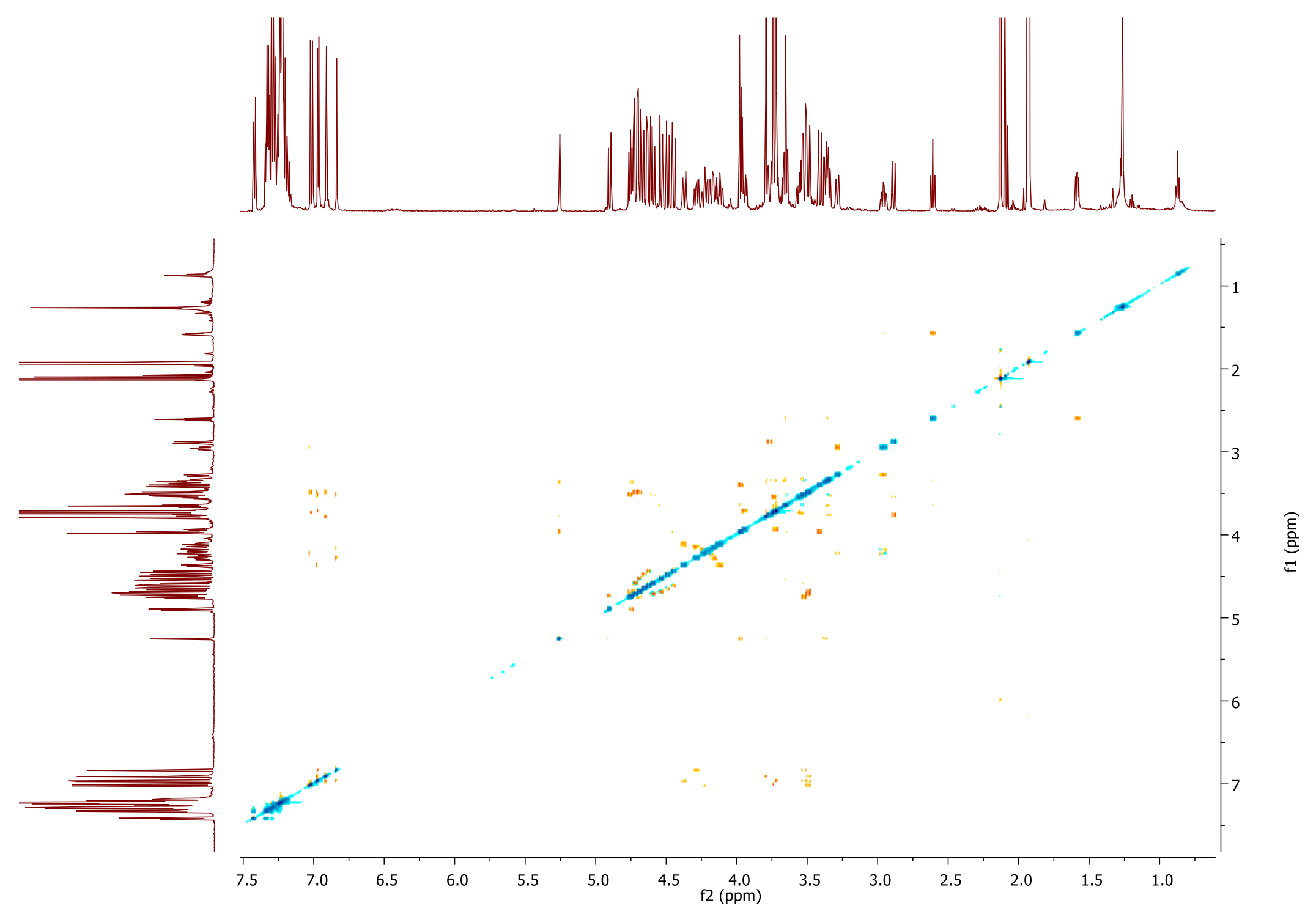

Figure S52. ${ }^{1} \mathrm{H}-{ }^{1} \mathrm{H}$ ROESY spectrum of compound $\boldsymbol{M - 8}$. 


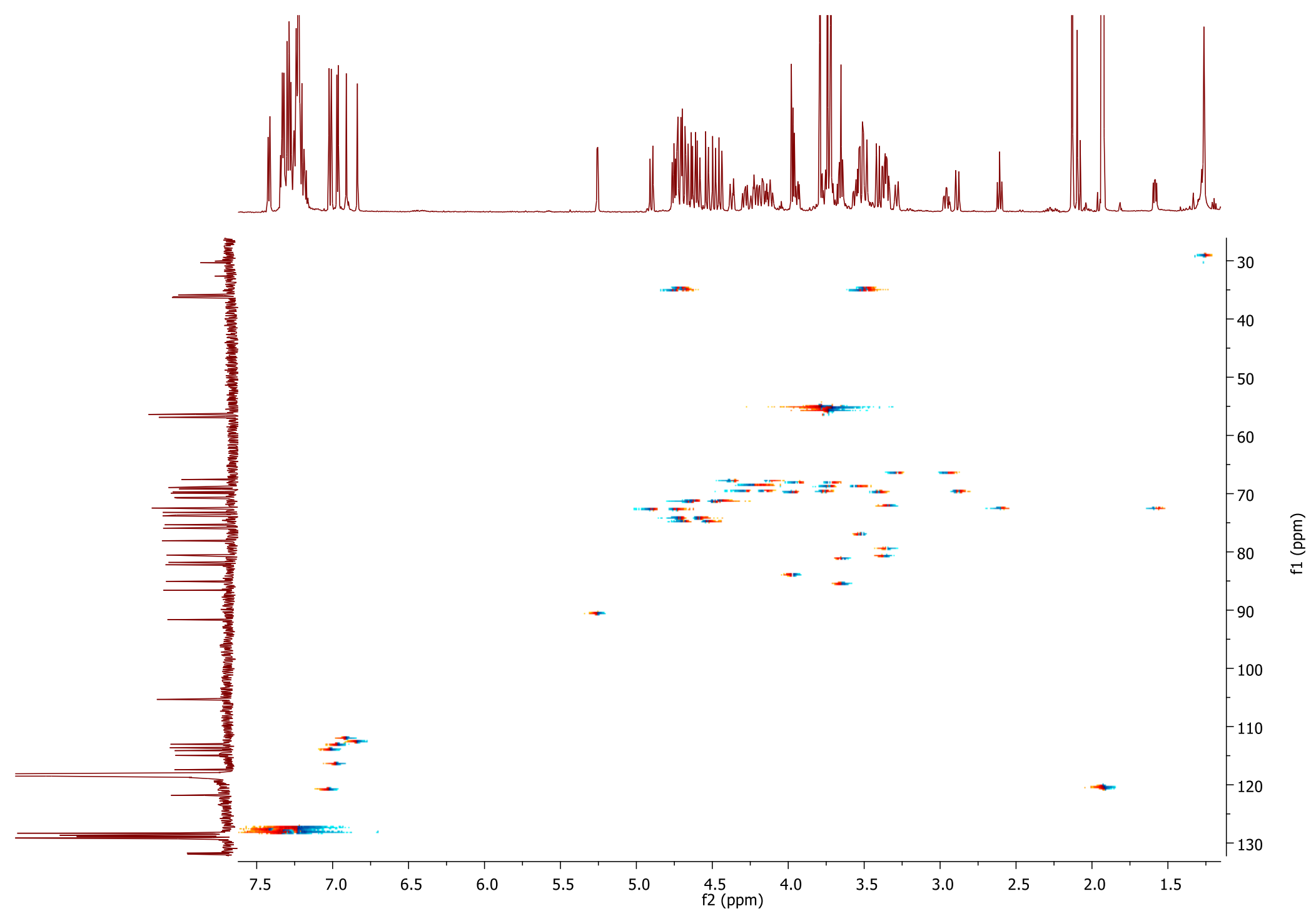

Figure S53. ${ }^{1} \mathrm{H}-{ }^{13} \mathrm{C}$ HSQC spectrum of compound $\boldsymbol{M - 8}$. 


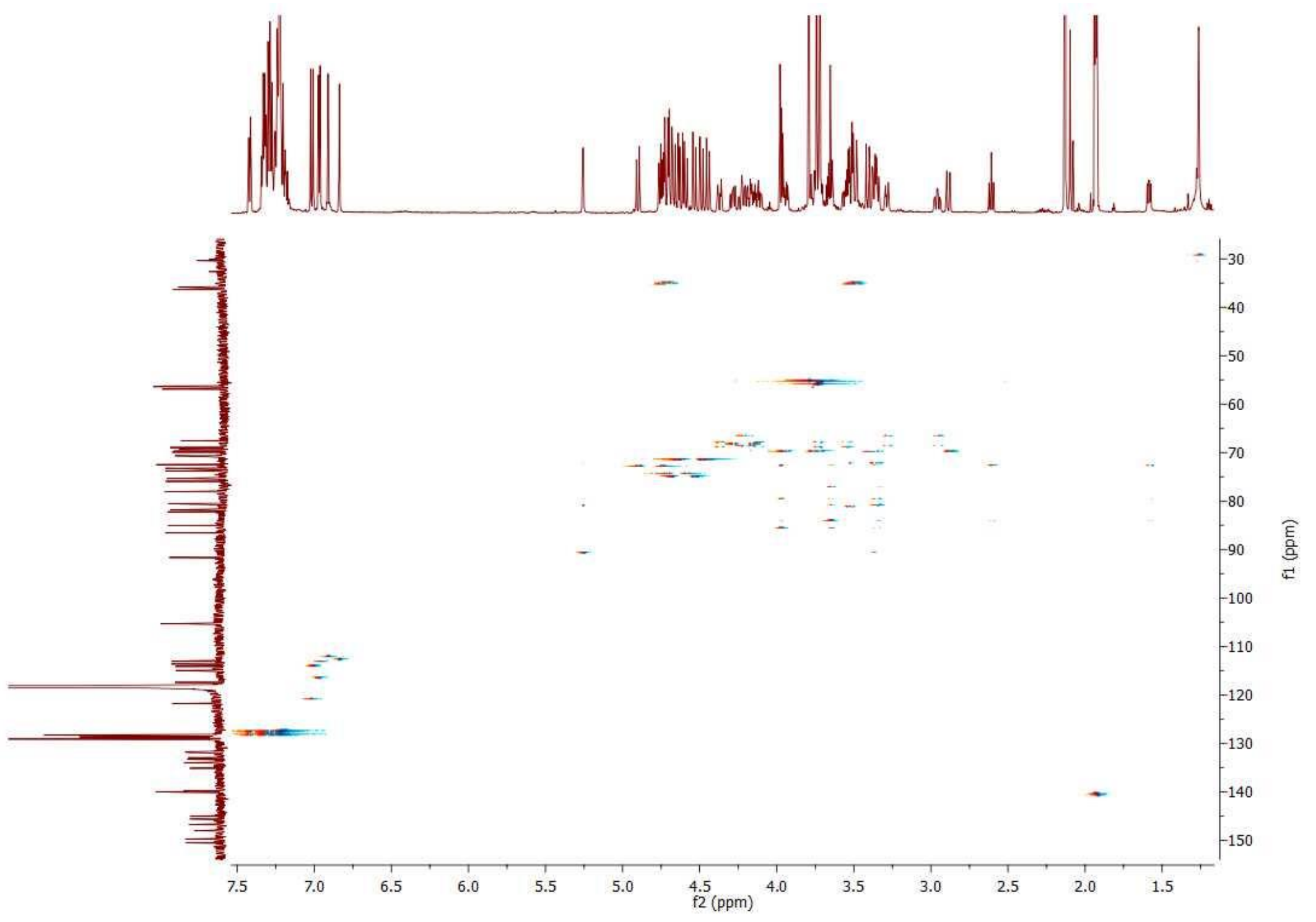

Figure S54. ${ }^{1} \mathrm{H}-{ }^{13} \mathrm{C}$ HSQCTOCSY spectrum of compound $\boldsymbol{M - 8}$. 


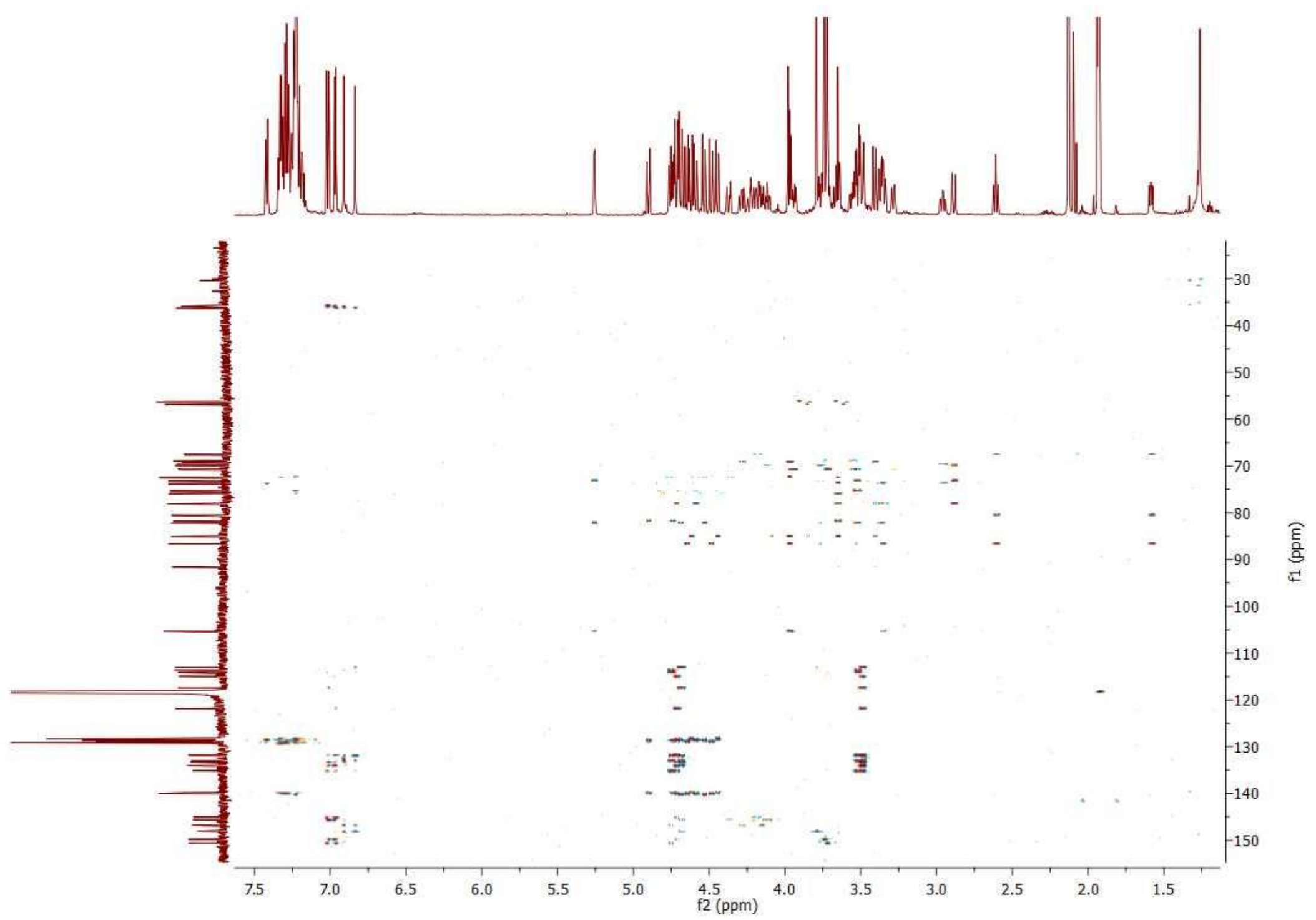

Figure S55. ${ }^{1} \mathrm{H}-{ }^{13} \mathrm{C}$ HMBC spectrum of compound $\boldsymbol{M - 8}$. 


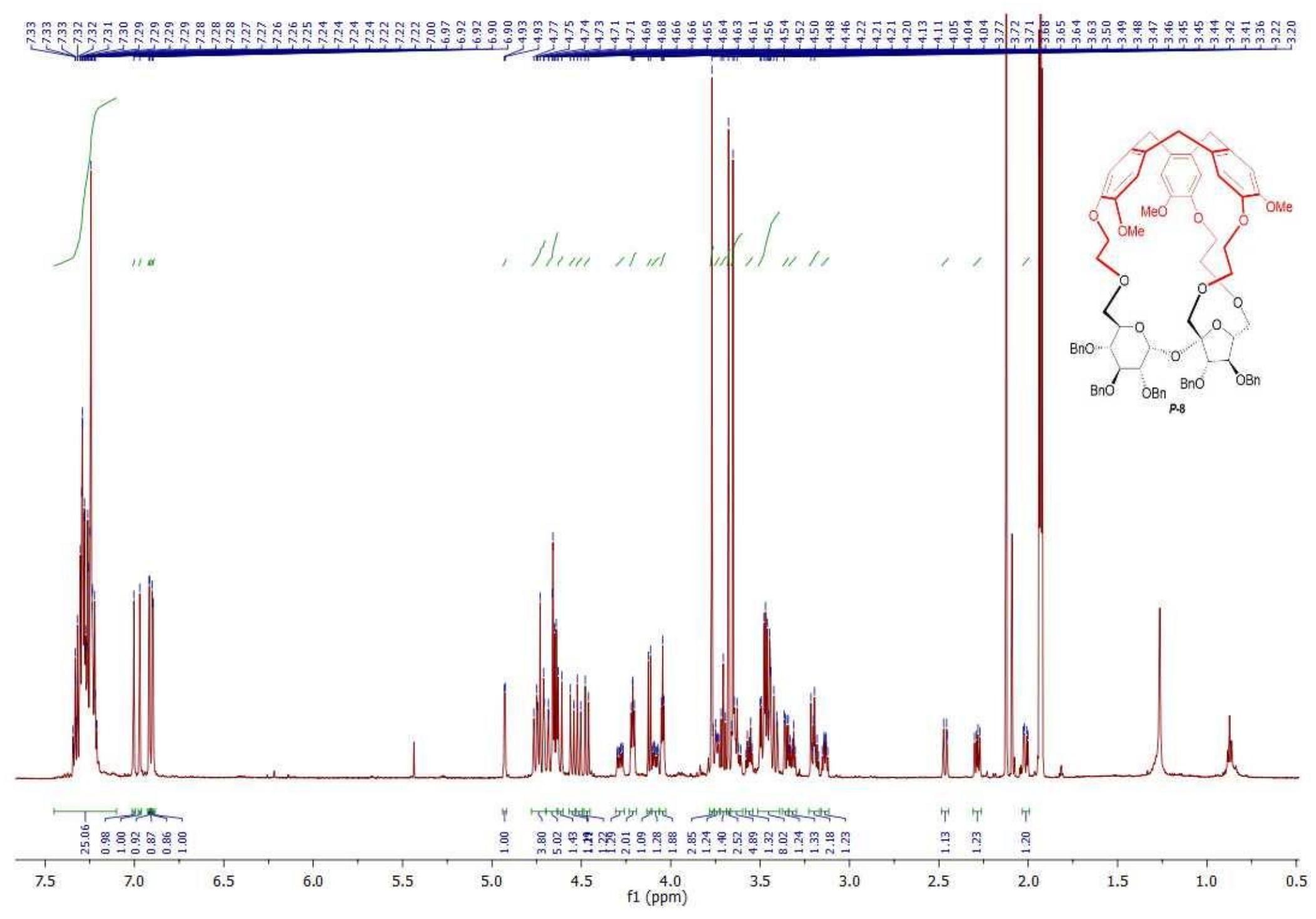

Figure S56. ${ }^{1} \mathrm{H}$ NMR spectrum of compound $\boldsymbol{P}$-8. 


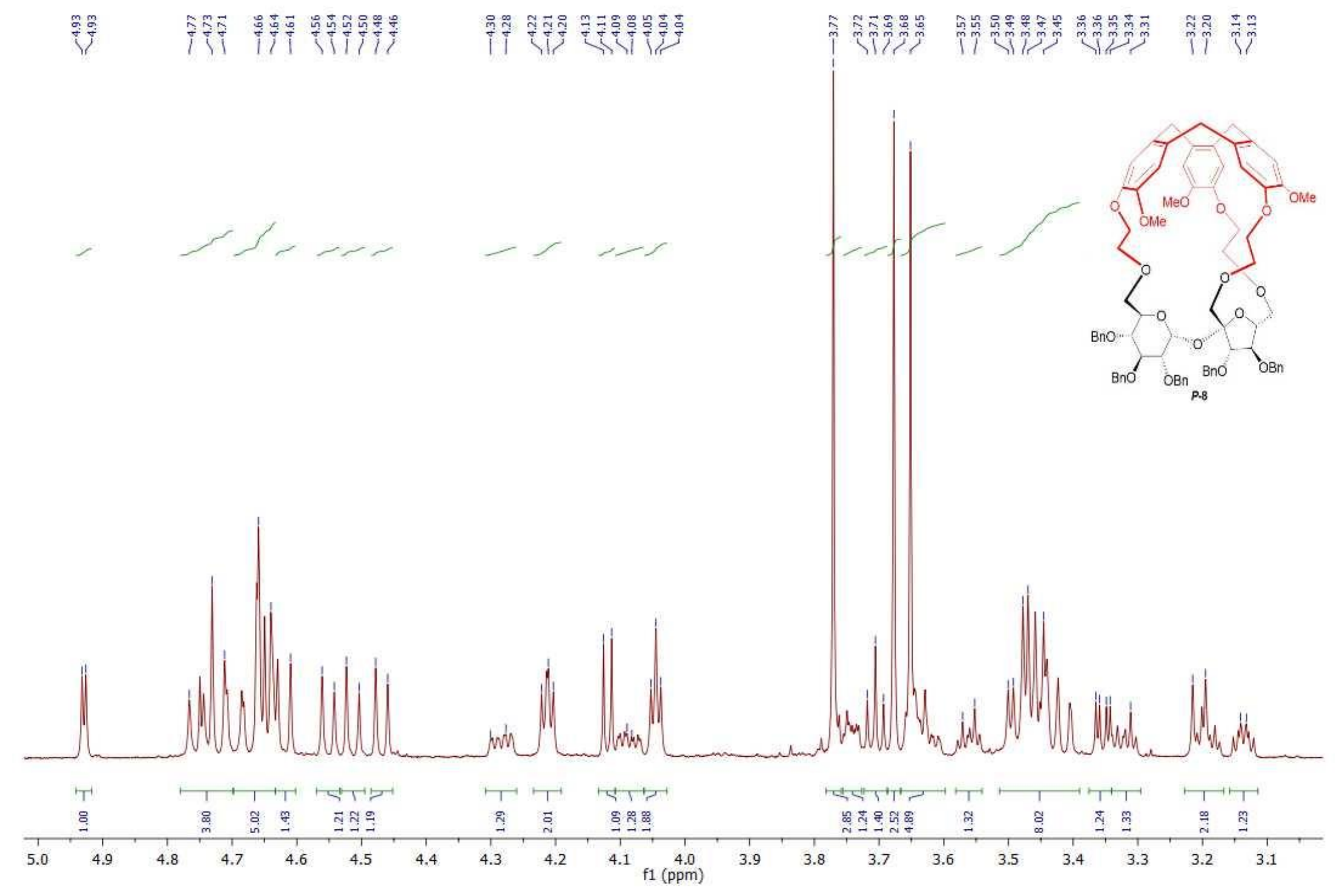

Figure S57. ${ }^{1} \mathrm{H}$ NMR spectrum of compound $\boldsymbol{P - 8}$ (aliphatic part). 


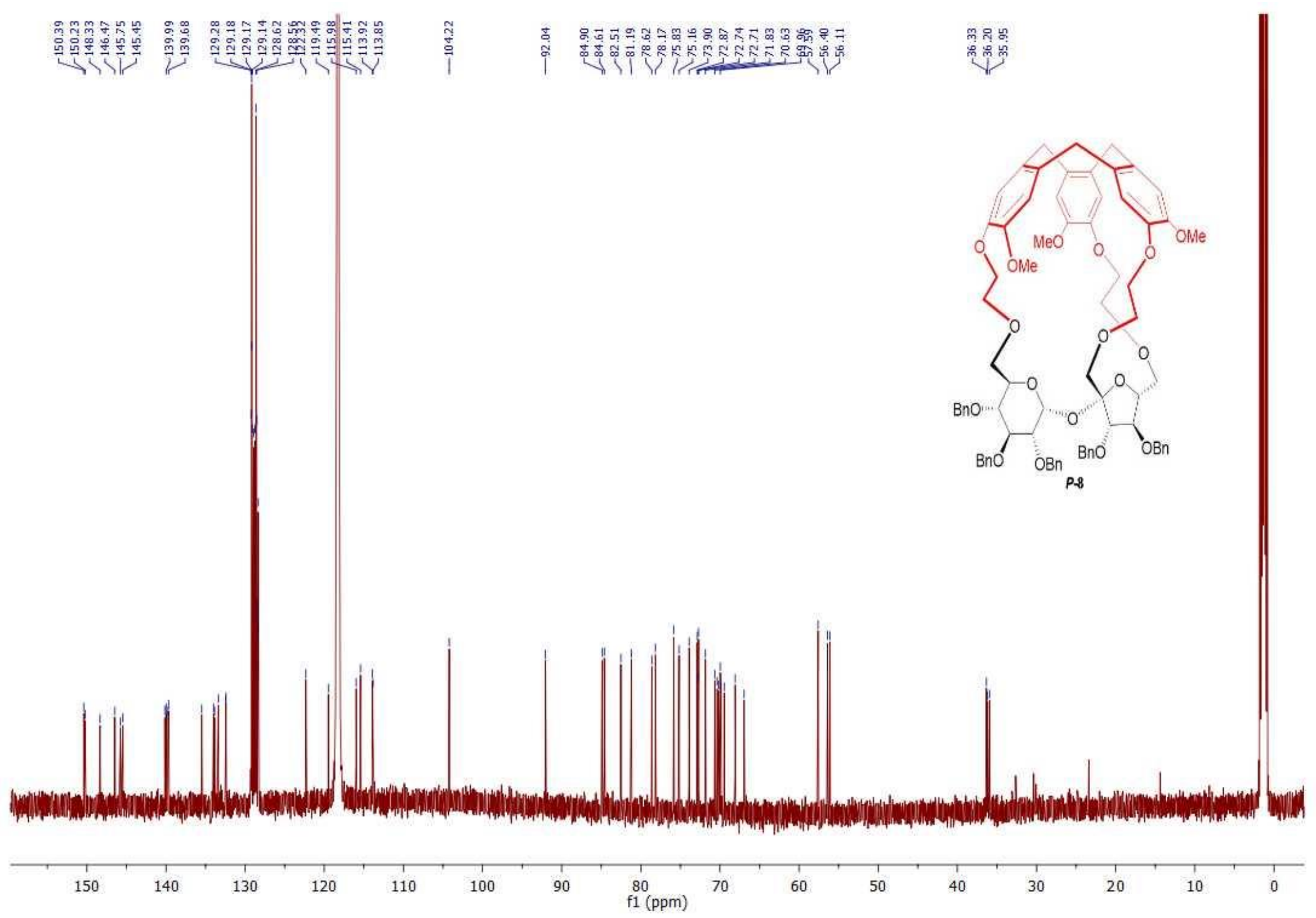

Figure S58. ${ }^{13} \mathrm{C}$ NMR spectrum of compound $\boldsymbol{P}-8$. 

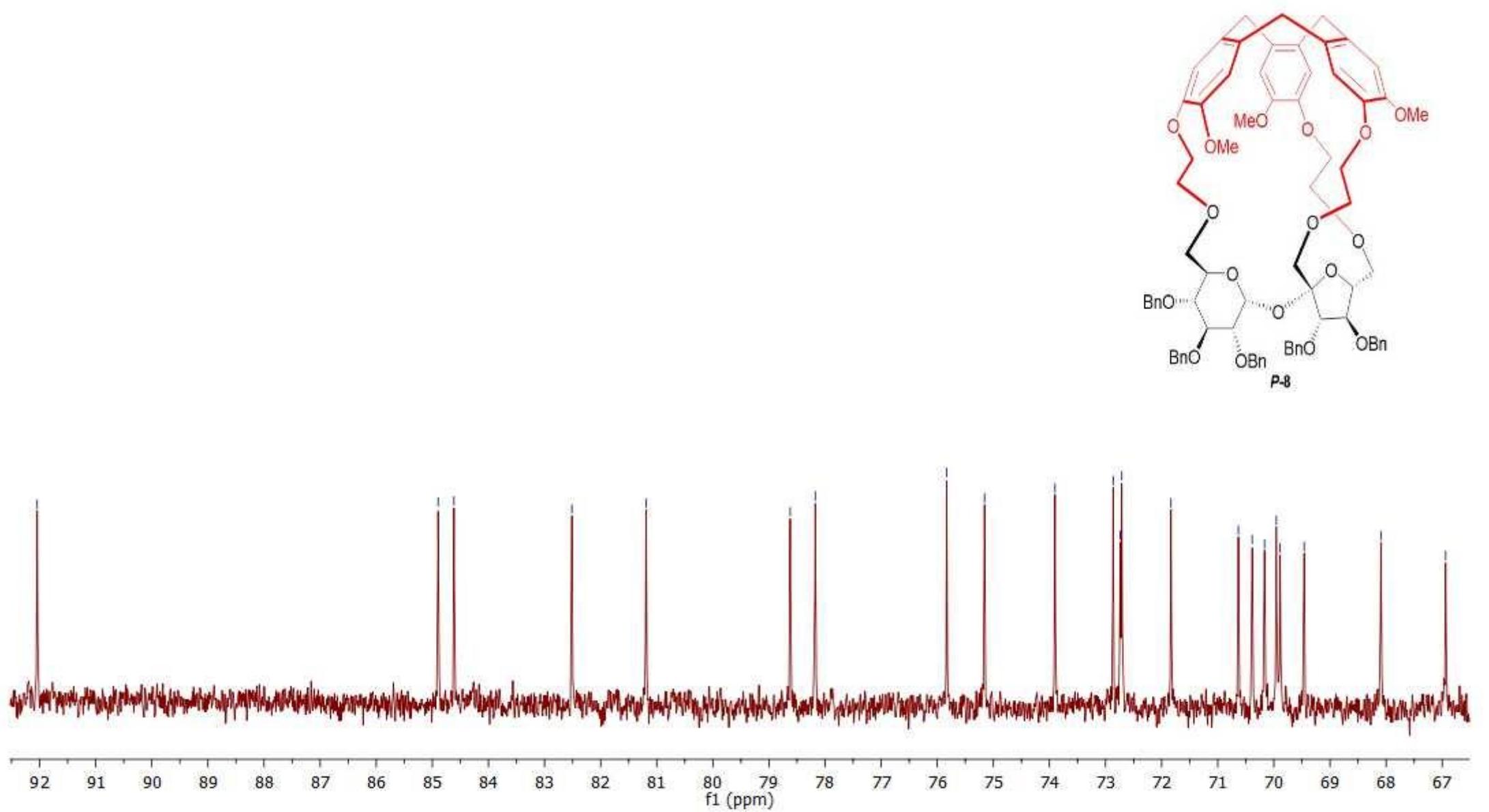

Figure S59. ${ }^{13} \mathrm{C}$ NMR spectrum of compound $\boldsymbol{P}-8$ (aliphatic part). 


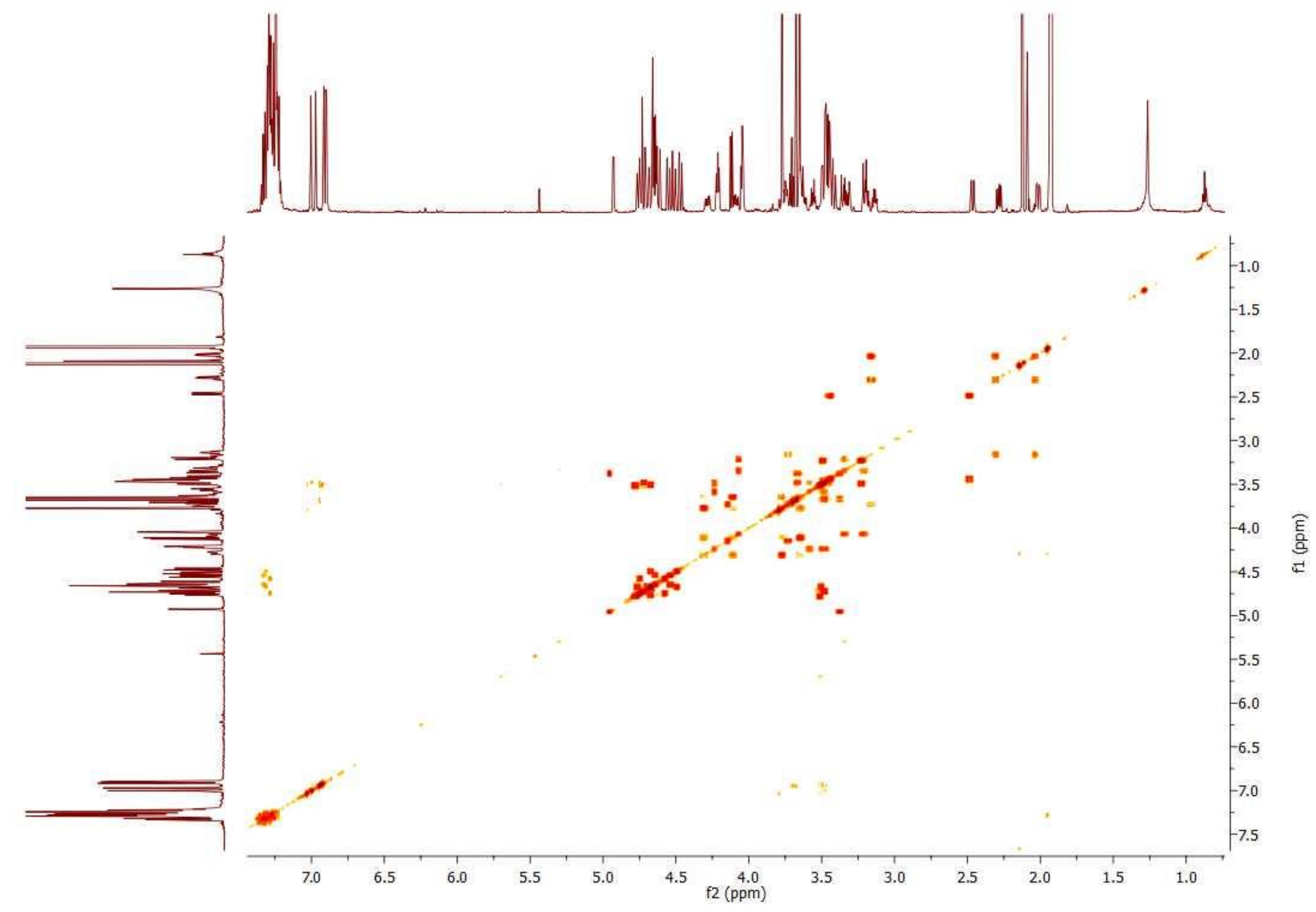

Figure S60. ${ }^{1} \mathrm{H}-{ }^{1} \mathrm{H}$ COSY spectrum of compound $\boldsymbol{P}-8$. 


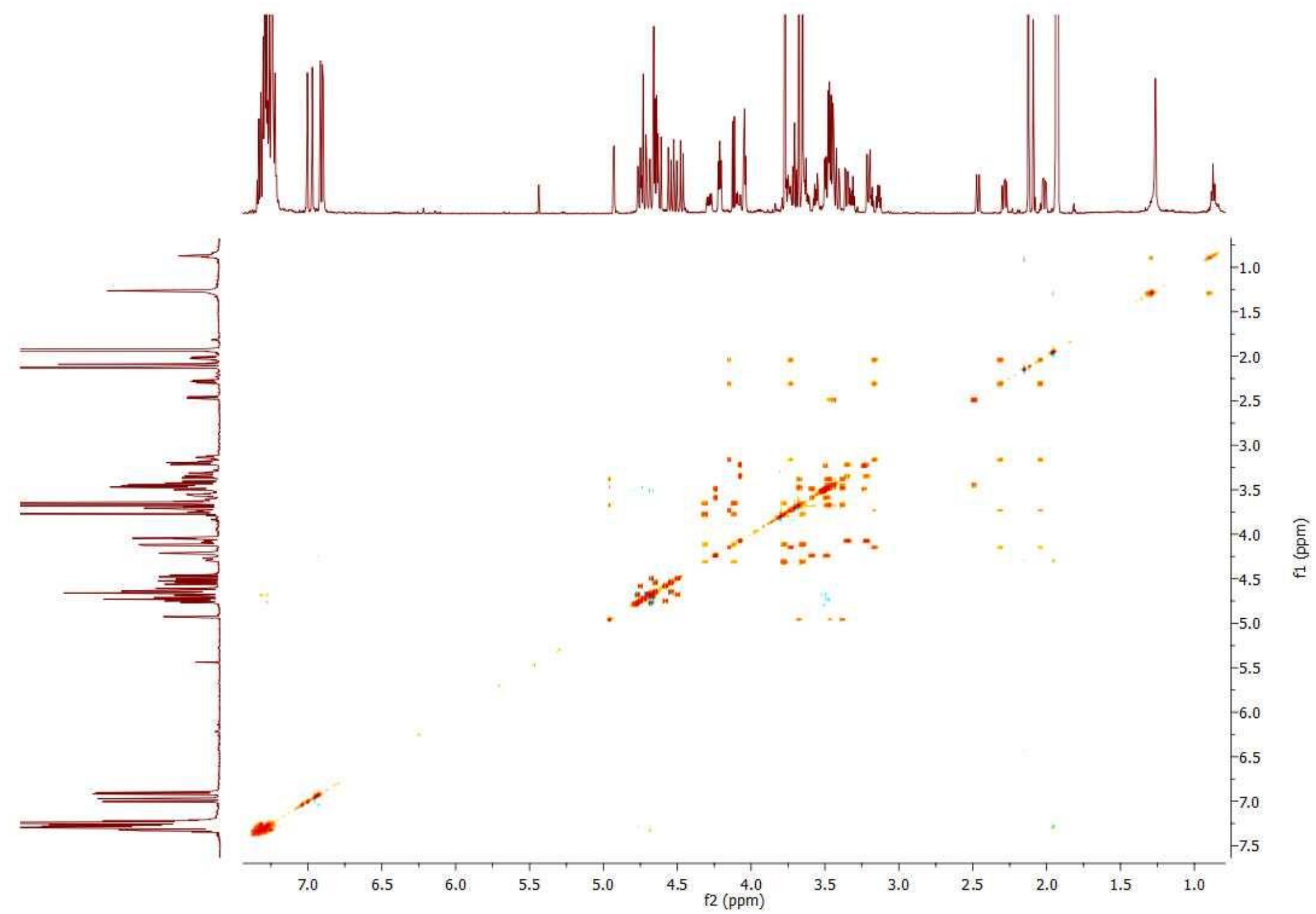

Figure S61. ${ }^{1} \mathrm{H}-{ }^{1} \mathrm{H}$ TOCSY spectrum of compound $\boldsymbol{P}-\mathbf{8}$. 


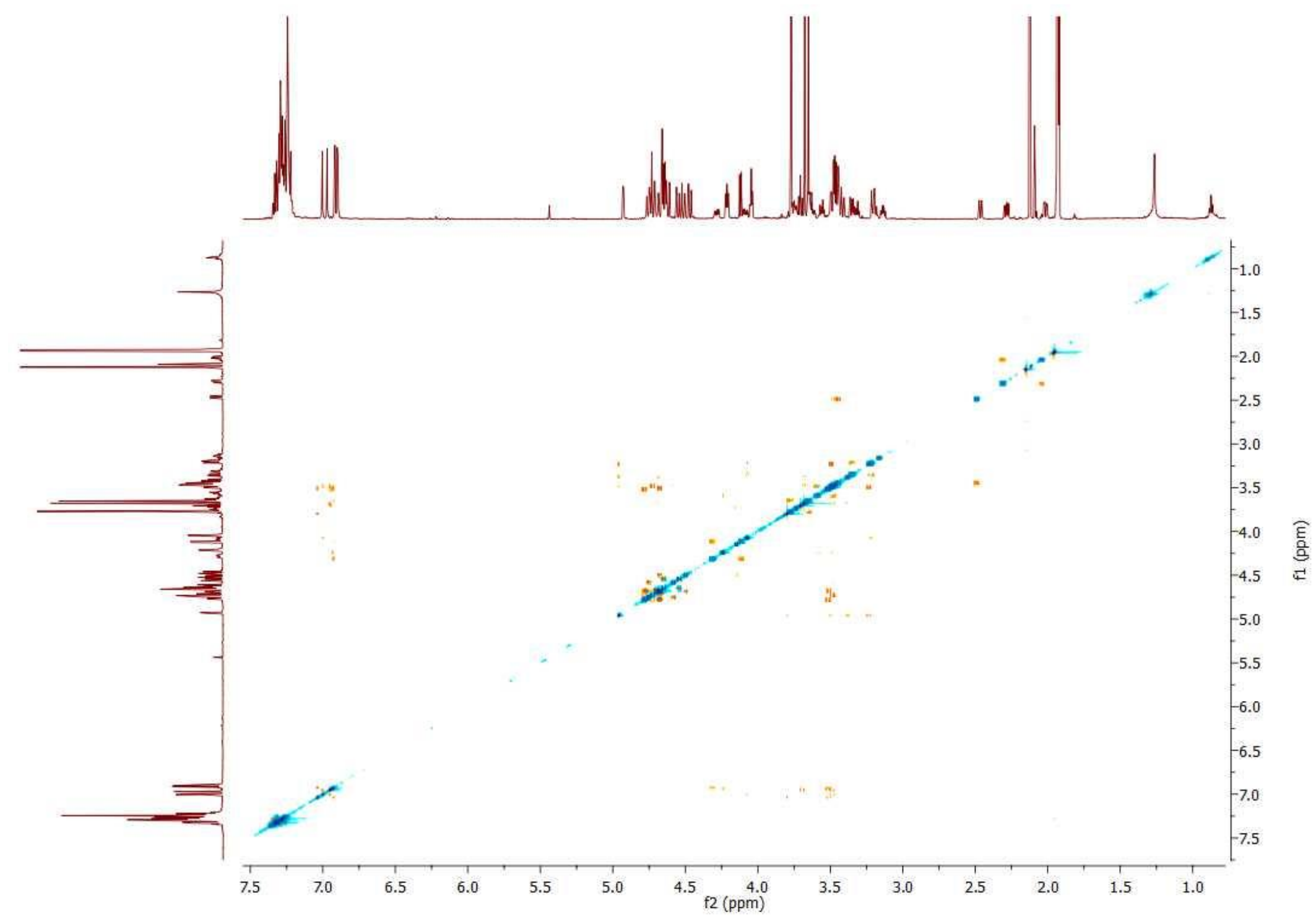

Figure S62. ${ }^{1} \mathrm{H}-{ }^{1} \mathrm{H}$ ROESY spectrum of compound $\boldsymbol{P}-\mathbf{8}$. 


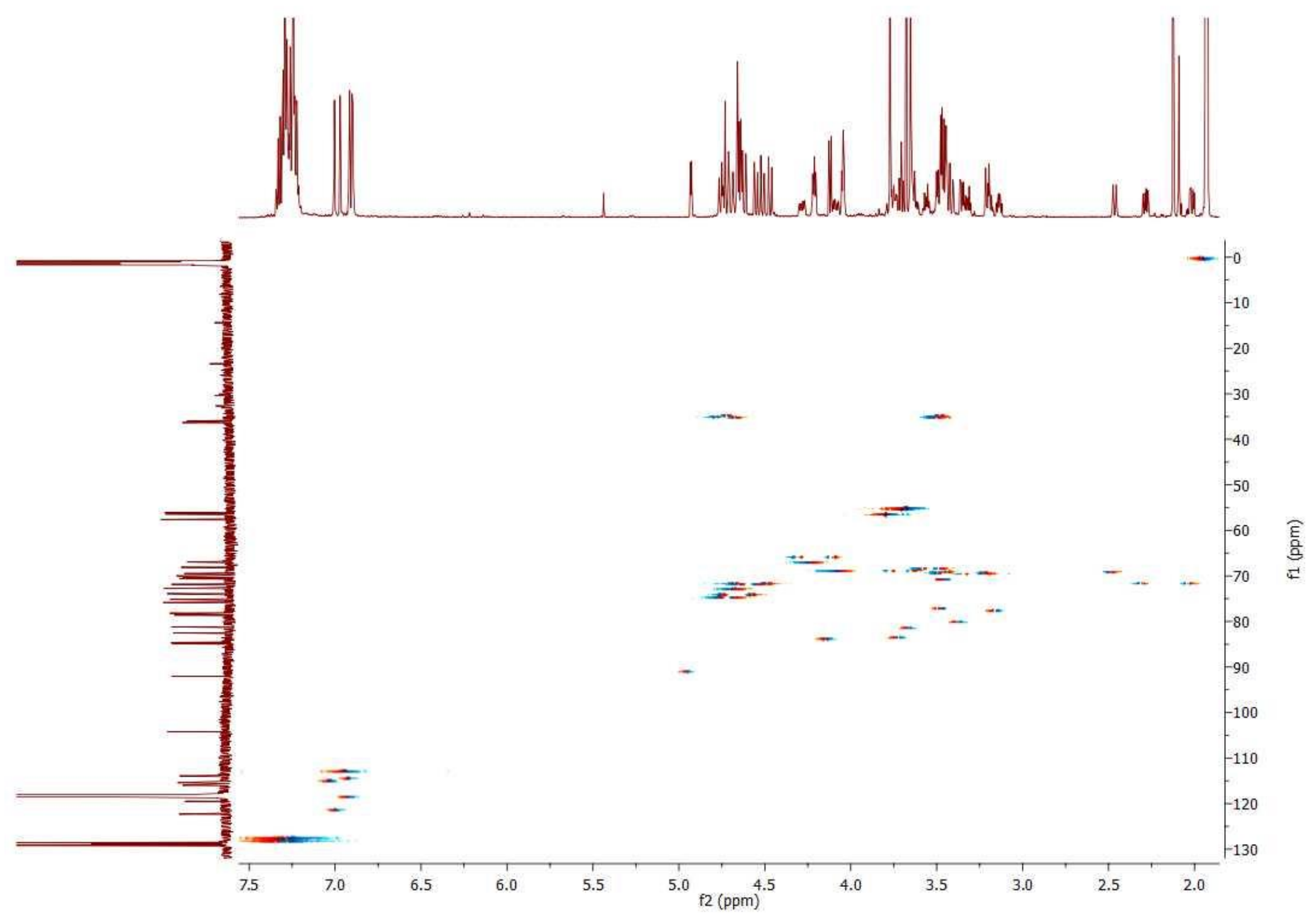

Figure S63. ${ }^{1} \mathrm{H}-{ }^{13} \mathrm{C}$ HSQC spectrum of compound $\boldsymbol{P}-\mathbf{8}$. 


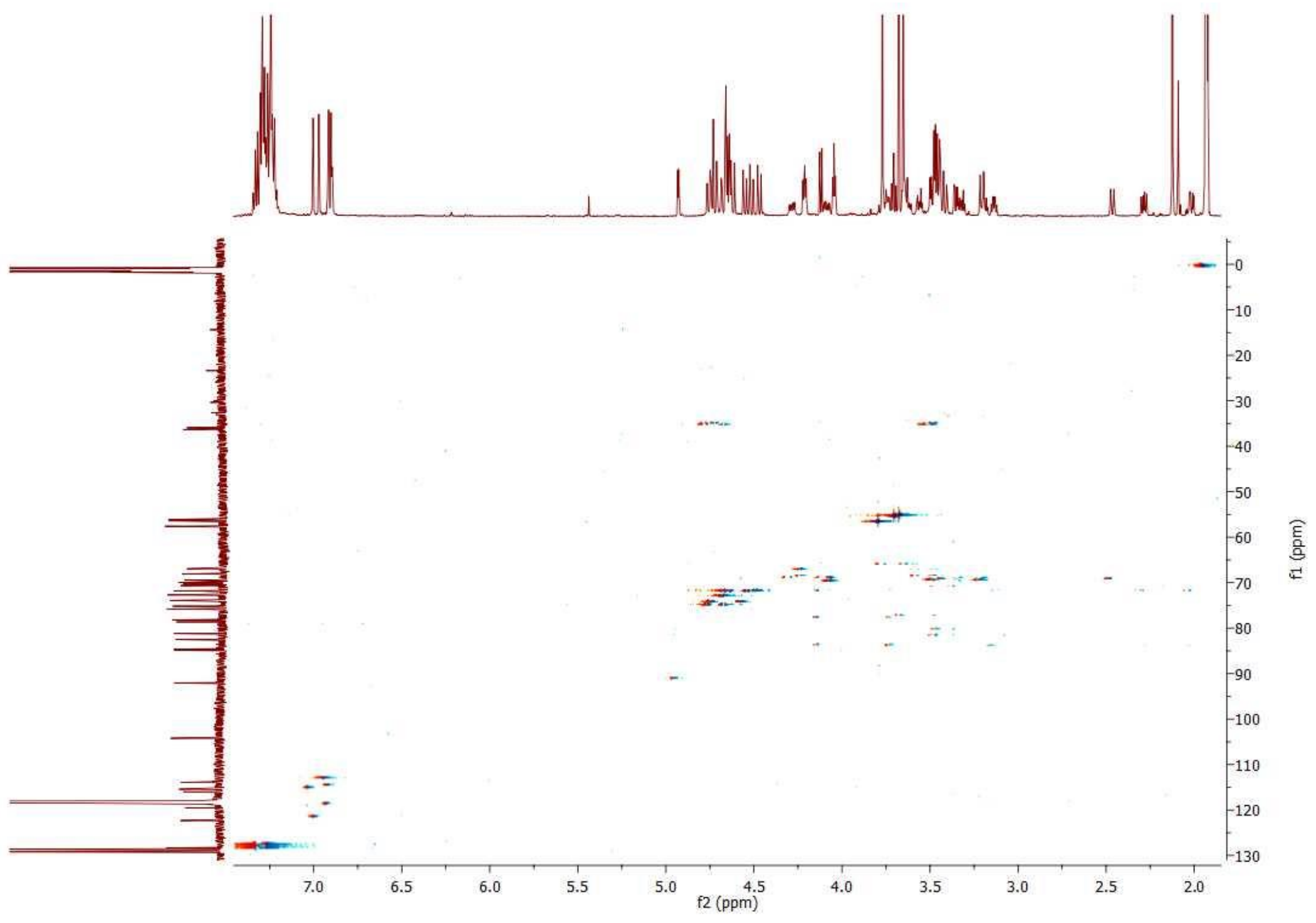

Figure S64. ${ }^{1} \mathrm{H}-{ }^{13} \mathrm{C}$ HSQC TOCSY spectrum of compound $\boldsymbol{P}-\mathbf{8}$. 


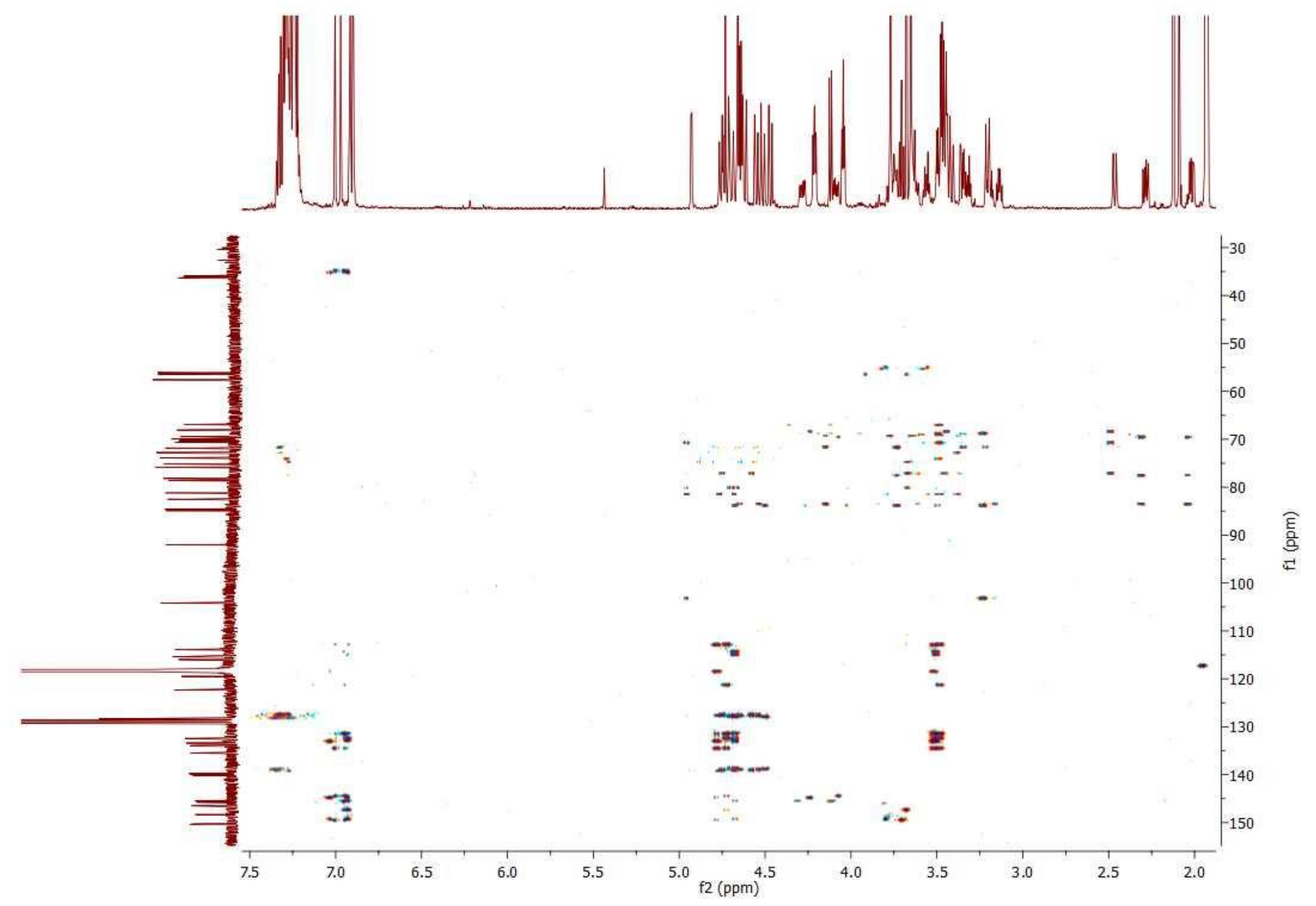

Figure S65. ${ }^{1} \mathrm{H}-{ }^{13} \mathrm{C}$ HMBC spectrum of compound $\boldsymbol{P}-\mathbf{8}$. 


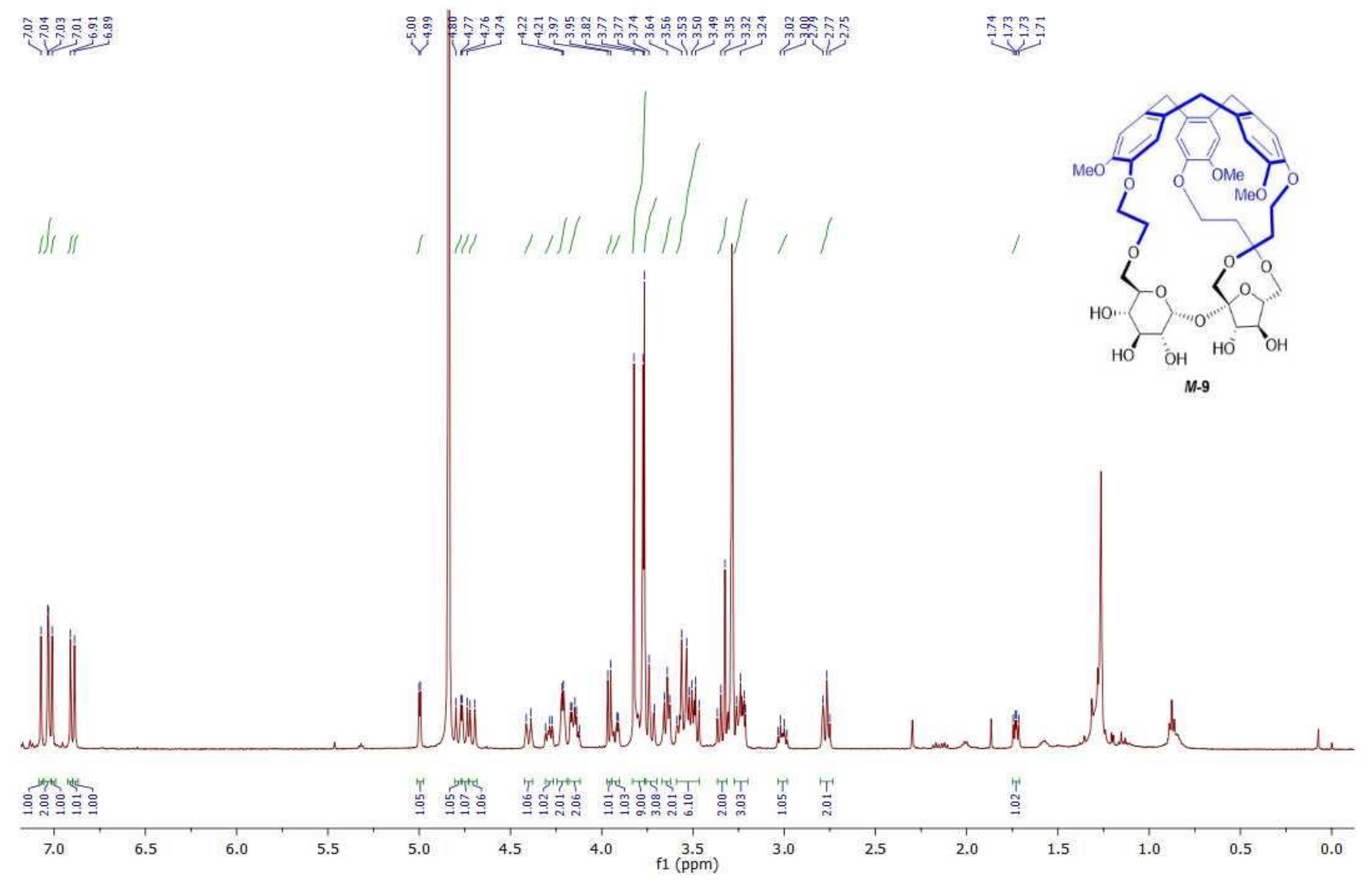

Figure S66. ${ }^{1} \mathrm{H}$ NMR spectrum of compound $\boldsymbol{M - 9}$. 


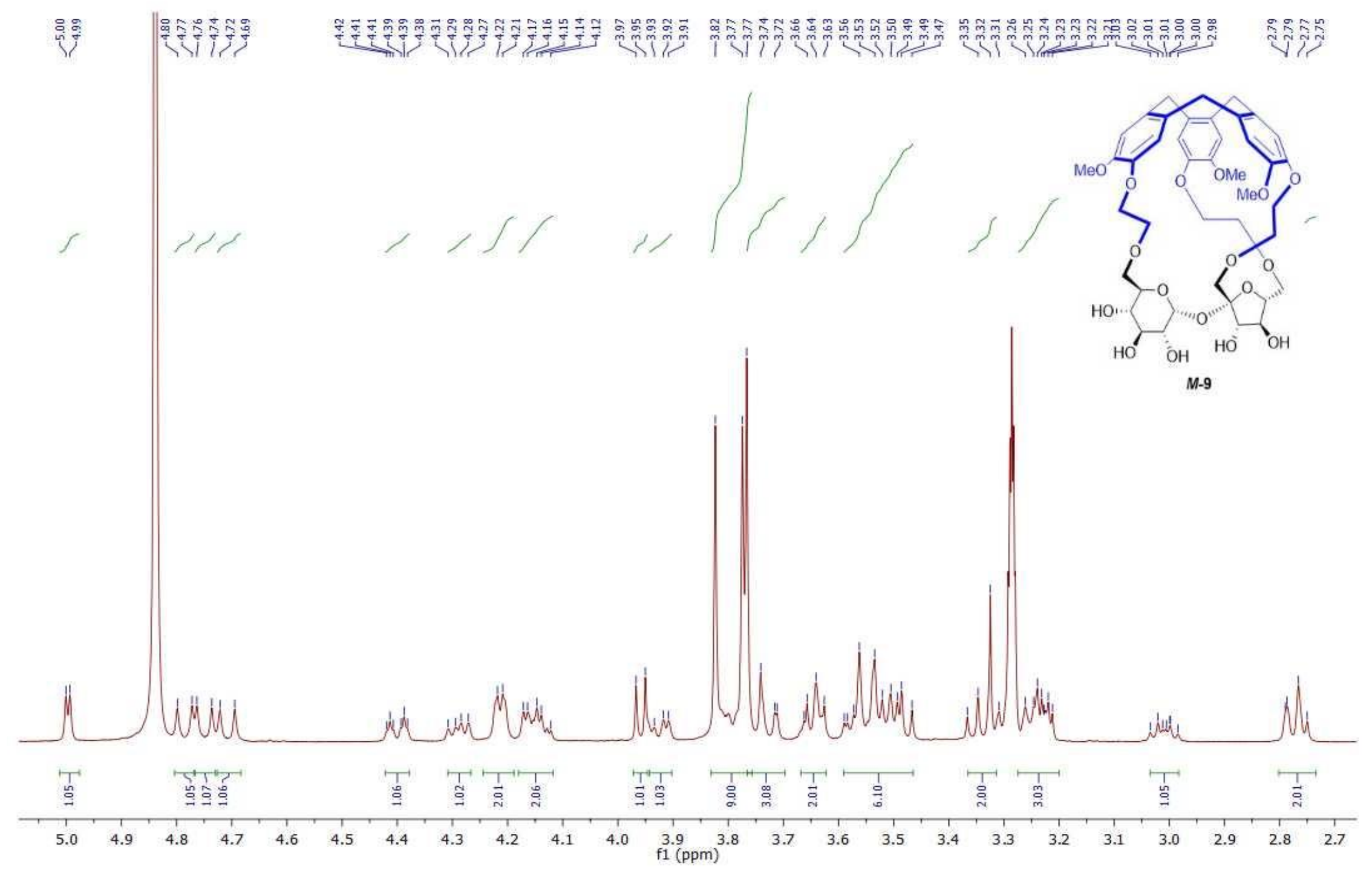

Figure S67. ${ }^{1} \mathrm{H}$ NMR spectrum of compound $\boldsymbol{M - 9}$ (aliphatic part). 

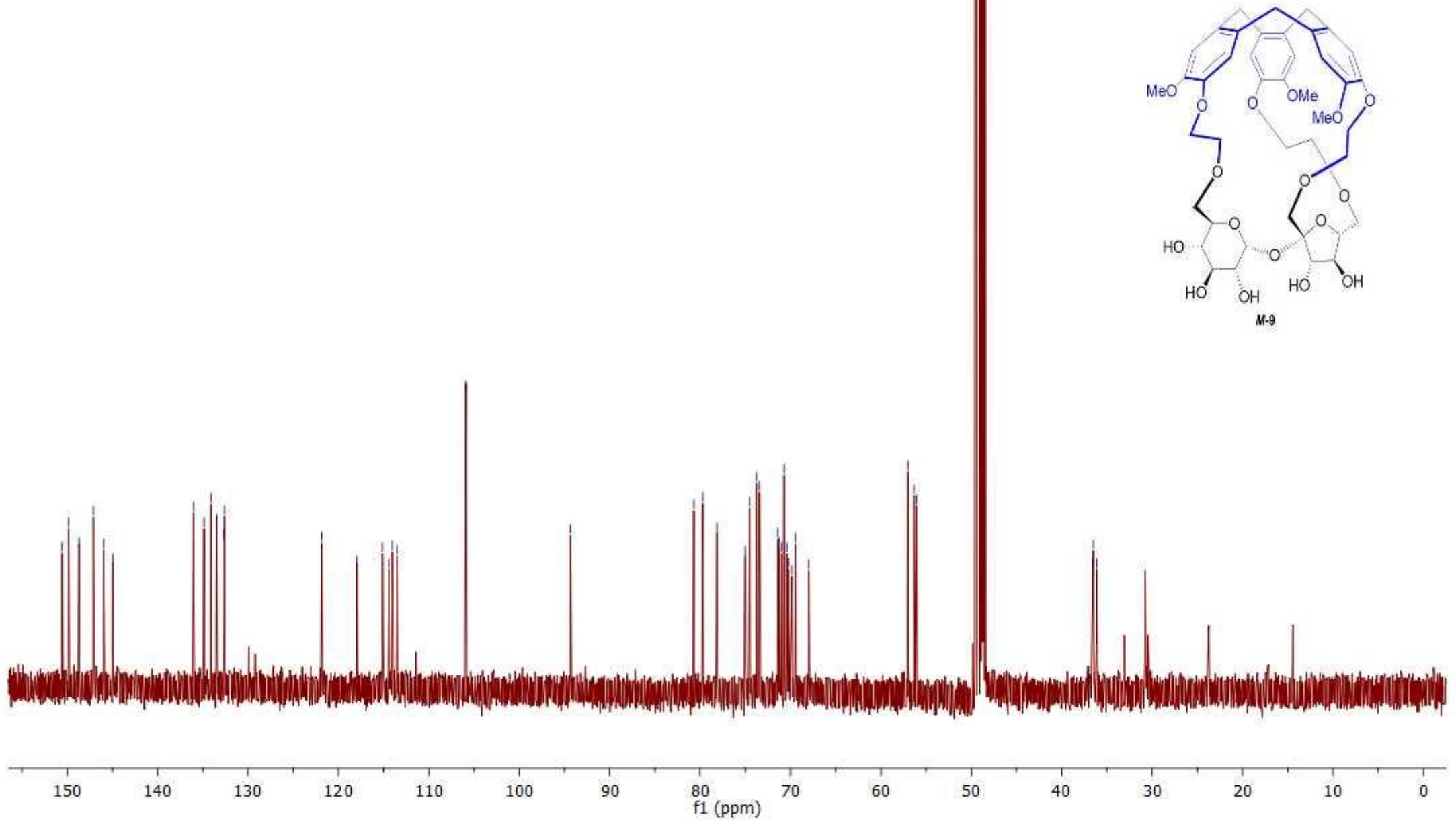

Figure S68. ${ }^{13} \mathrm{C}$ NMR spectrum of compound $\boldsymbol{M - 9}$. 

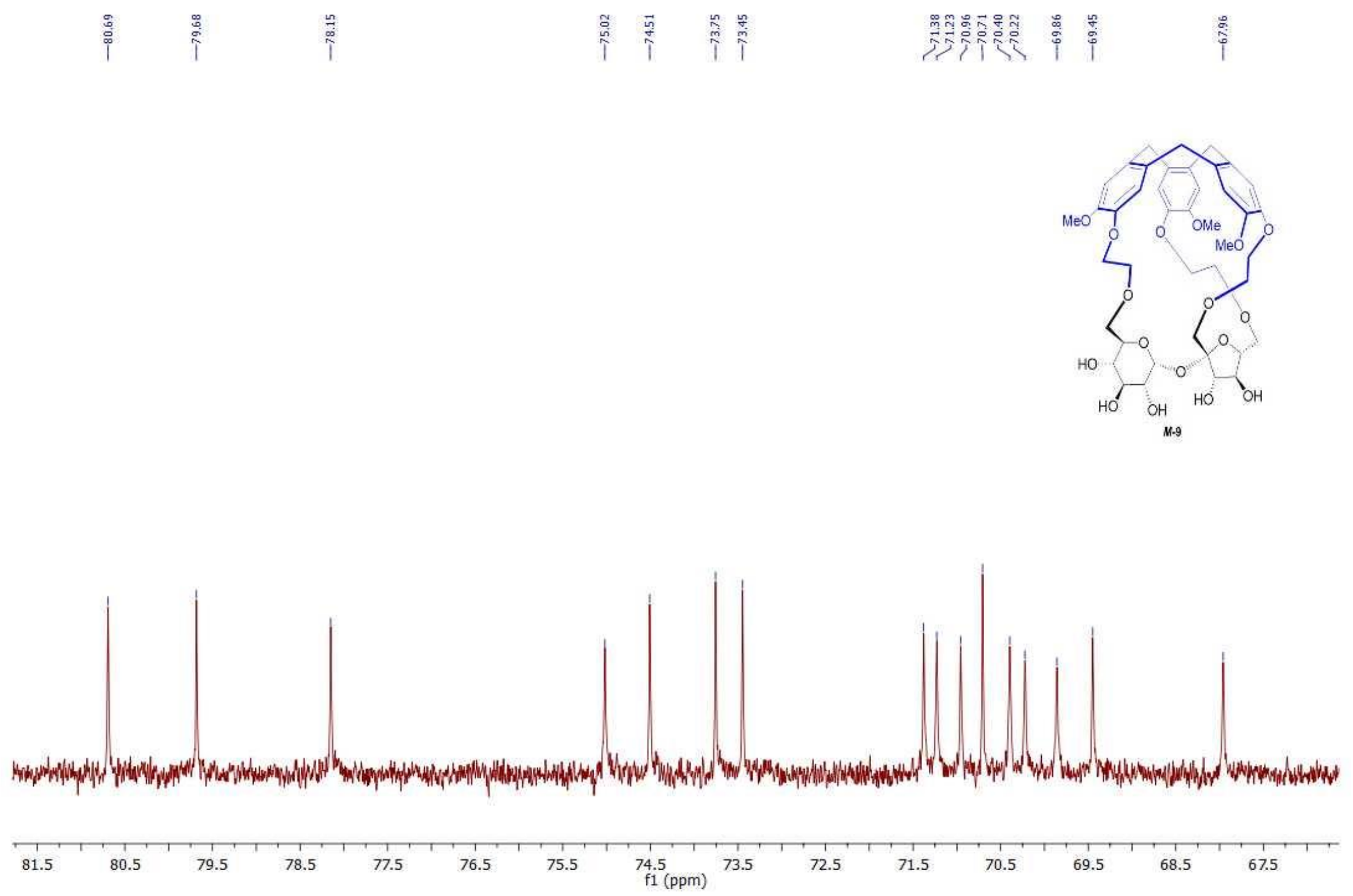

Figure S69. ${ }^{13} \mathrm{C}$ NMR spectrum of compound $\boldsymbol{M - 9}$ (aliphatic part). 


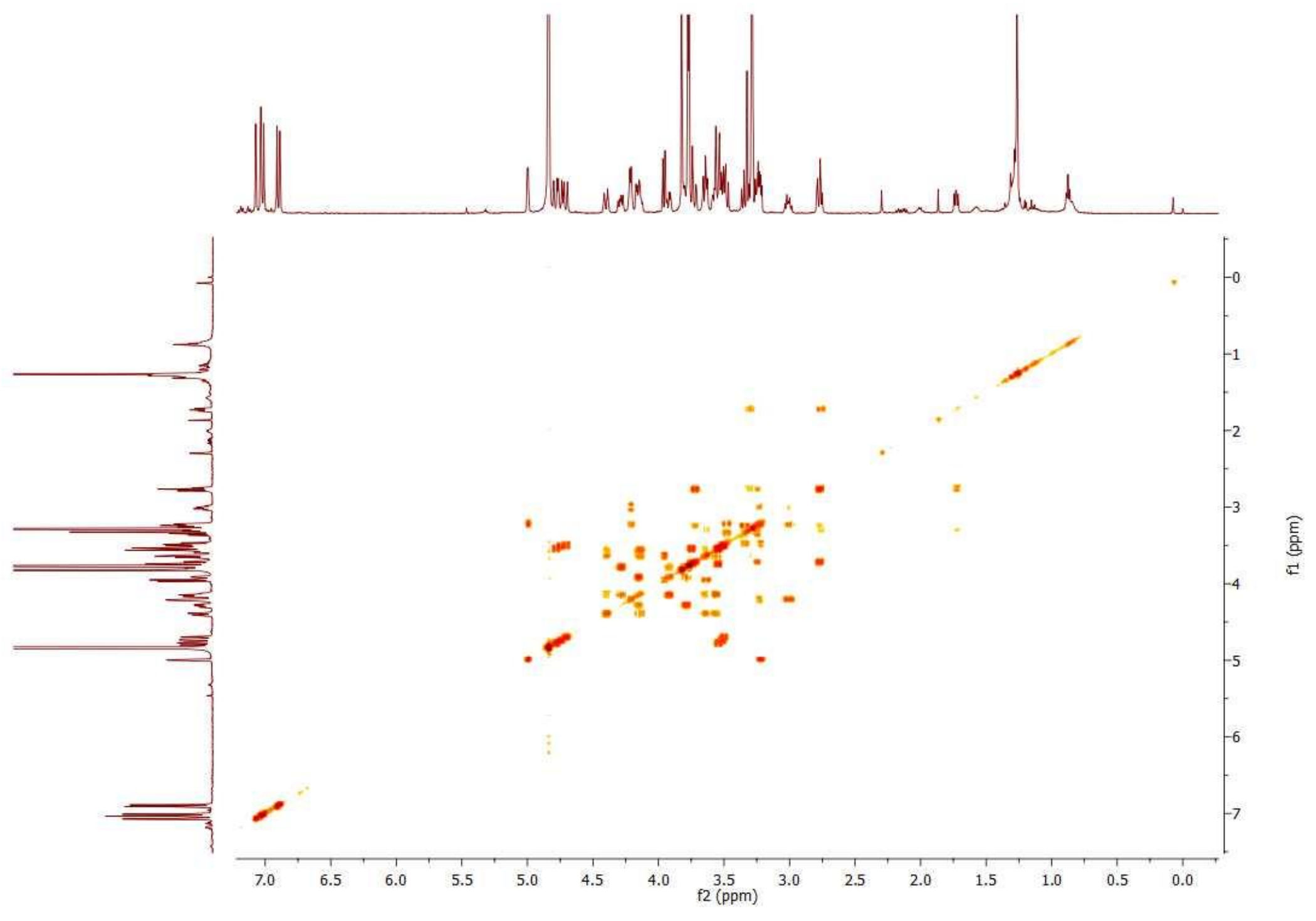

Figure S70. ${ }^{1} \mathrm{H}-{ }^{1} \mathrm{H}$ COSY spectrum of compound $\boldsymbol{M - 9}$. 


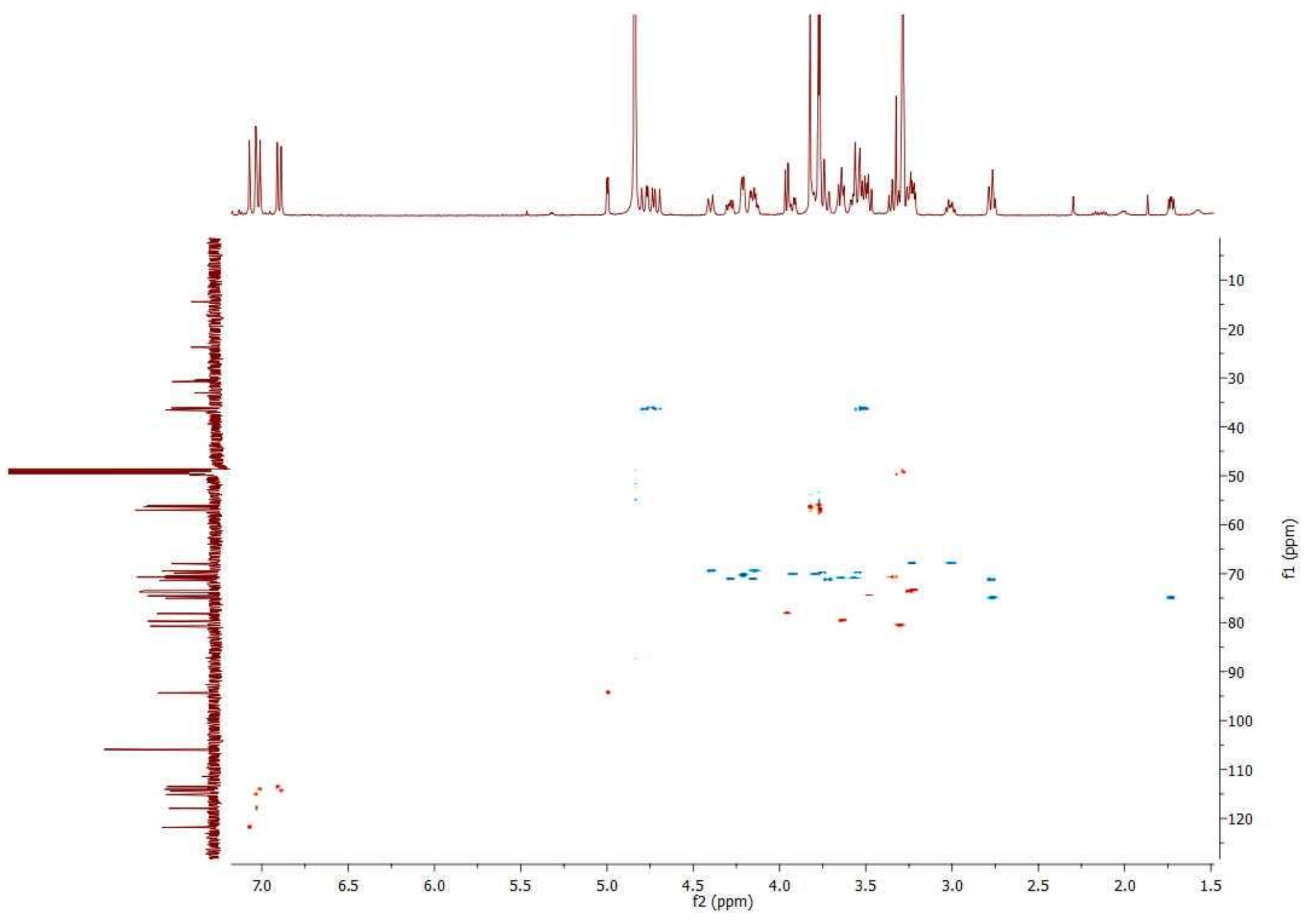

Figure S71. ${ }^{1} \mathrm{H}-{ }^{13} \mathrm{C}$ HSQC spectrum of compound $\boldsymbol{M - 9}$. 


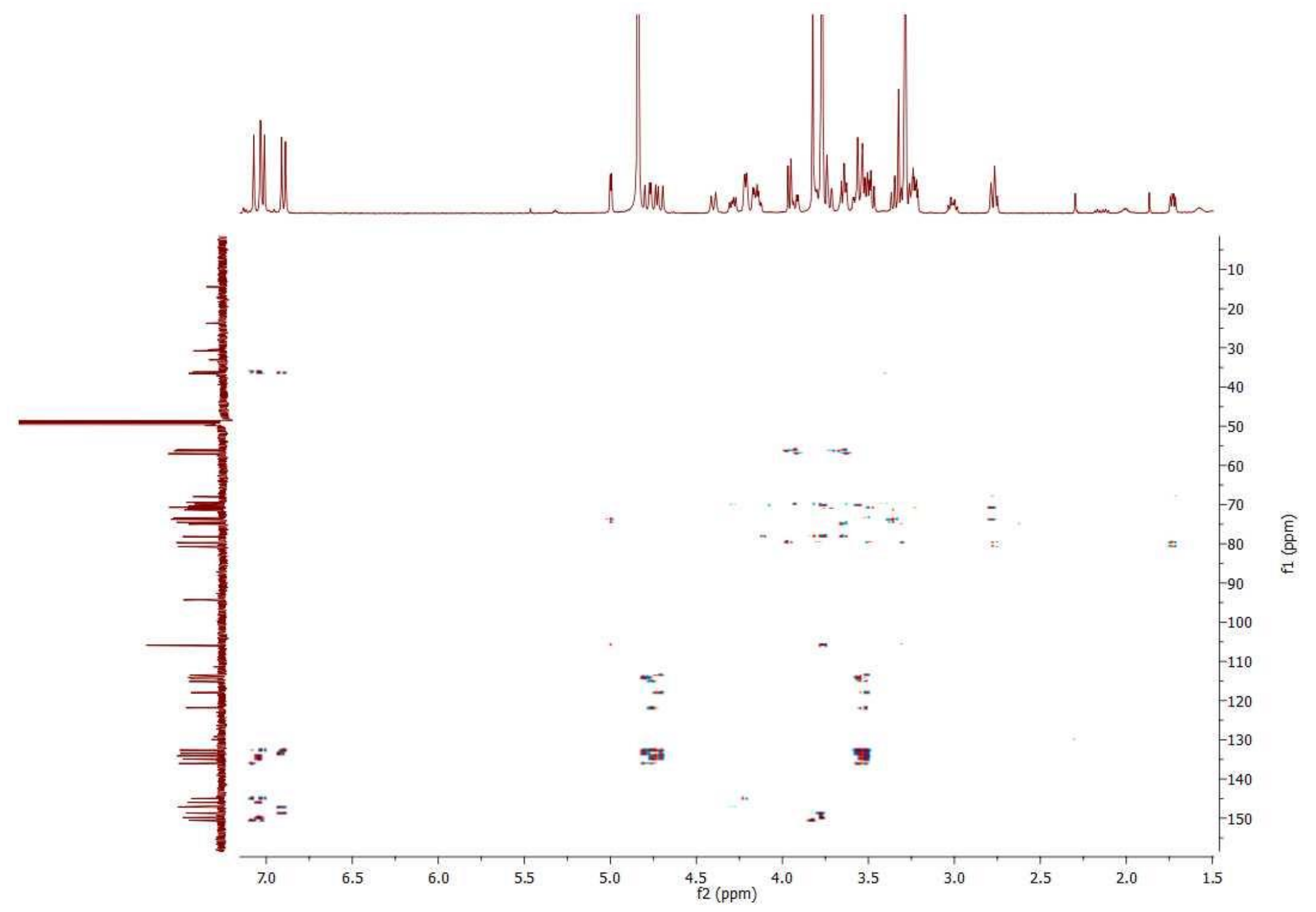

Figure S72. ${ }^{1} \mathrm{H}-{ }^{13} \mathrm{C}$ HMBC spectrum of compound $\boldsymbol{M - 9}$. 


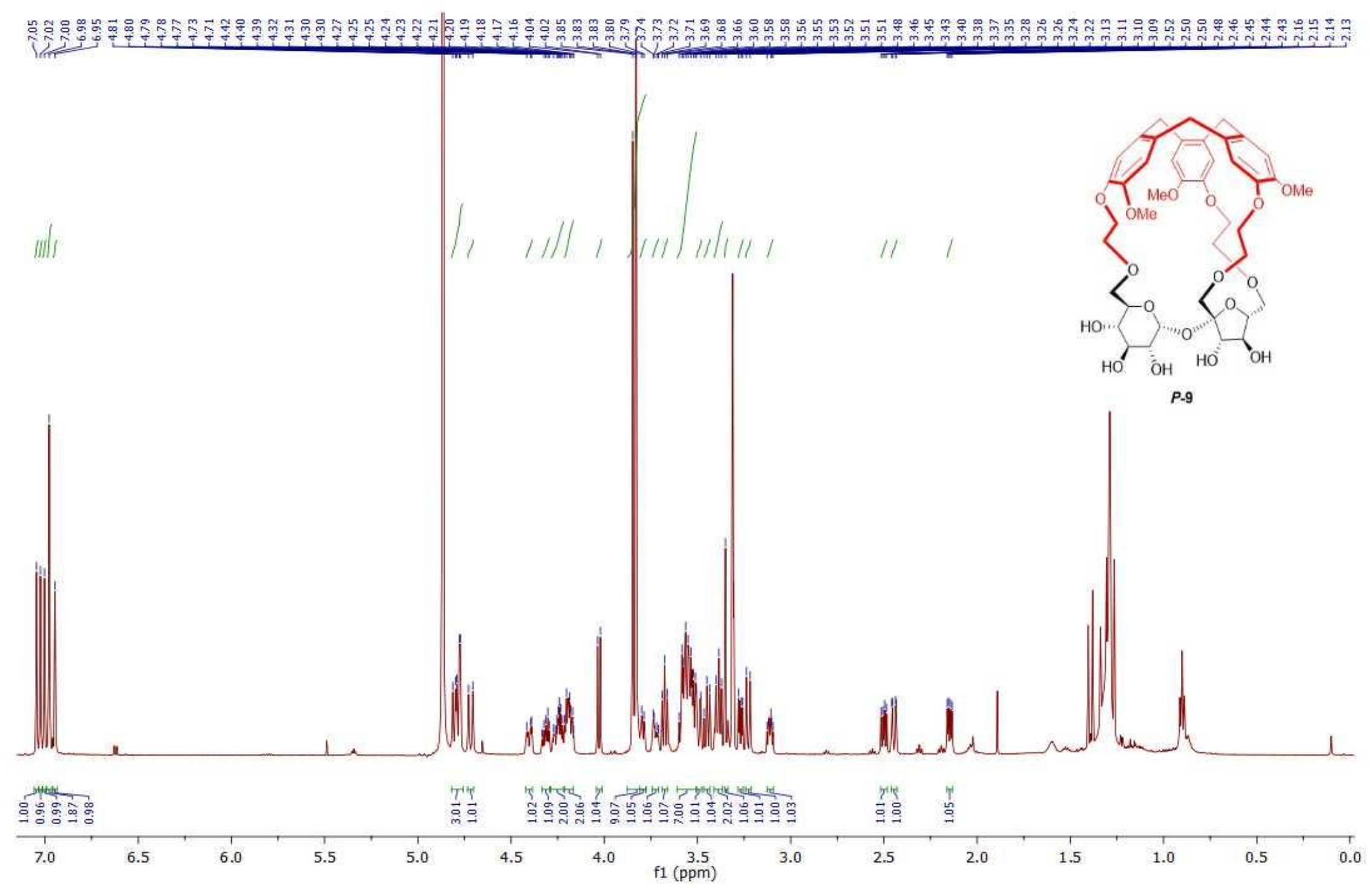

Figure S73. ${ }^{1} \mathrm{H}$ NMR spectrum of compound $\boldsymbol{P}$-9. 


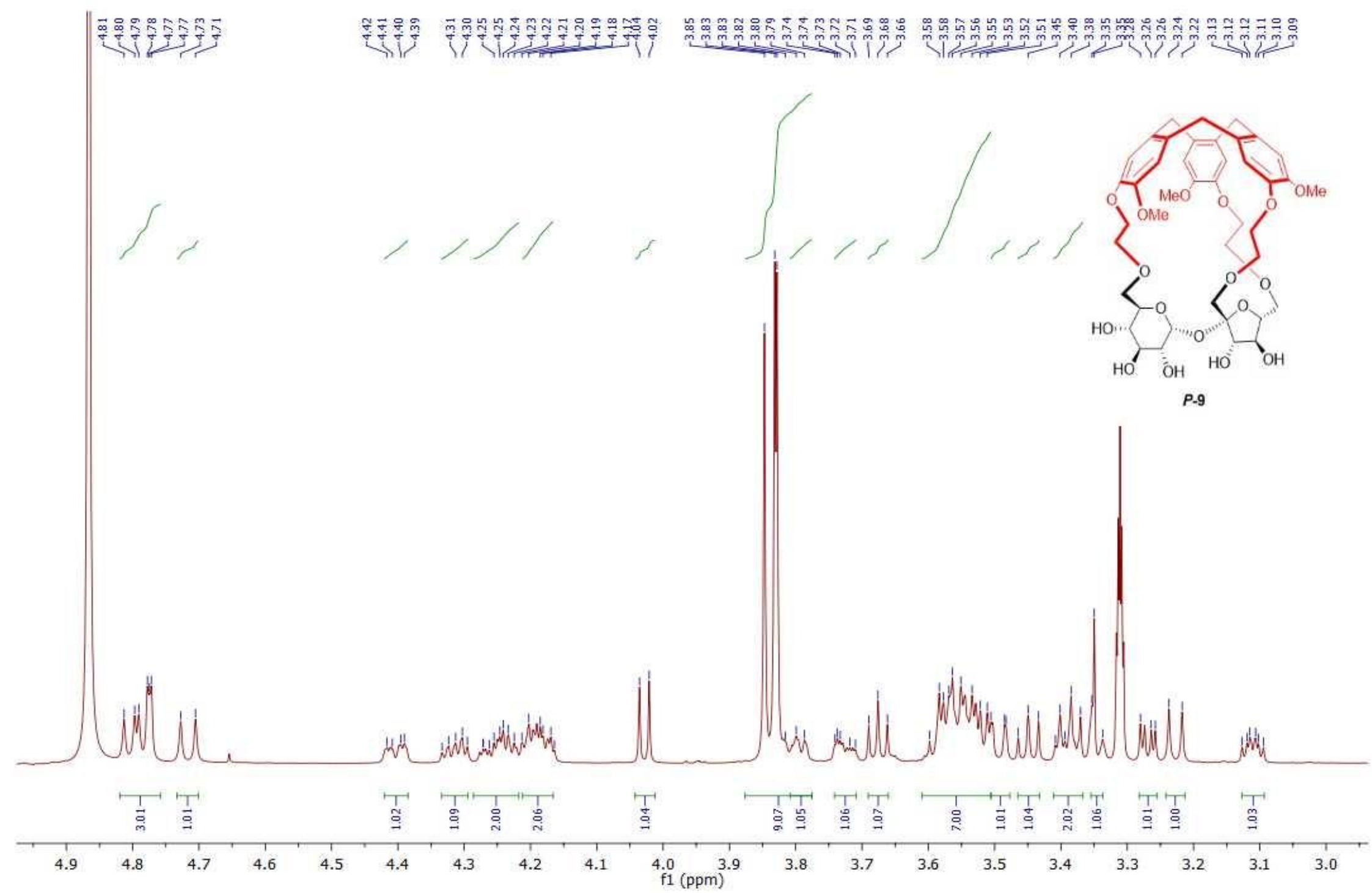

Figure S74. ${ }^{1} \mathrm{H}$ NMR spectrum of compound $\boldsymbol{P}-9$ (aliphatic part). 

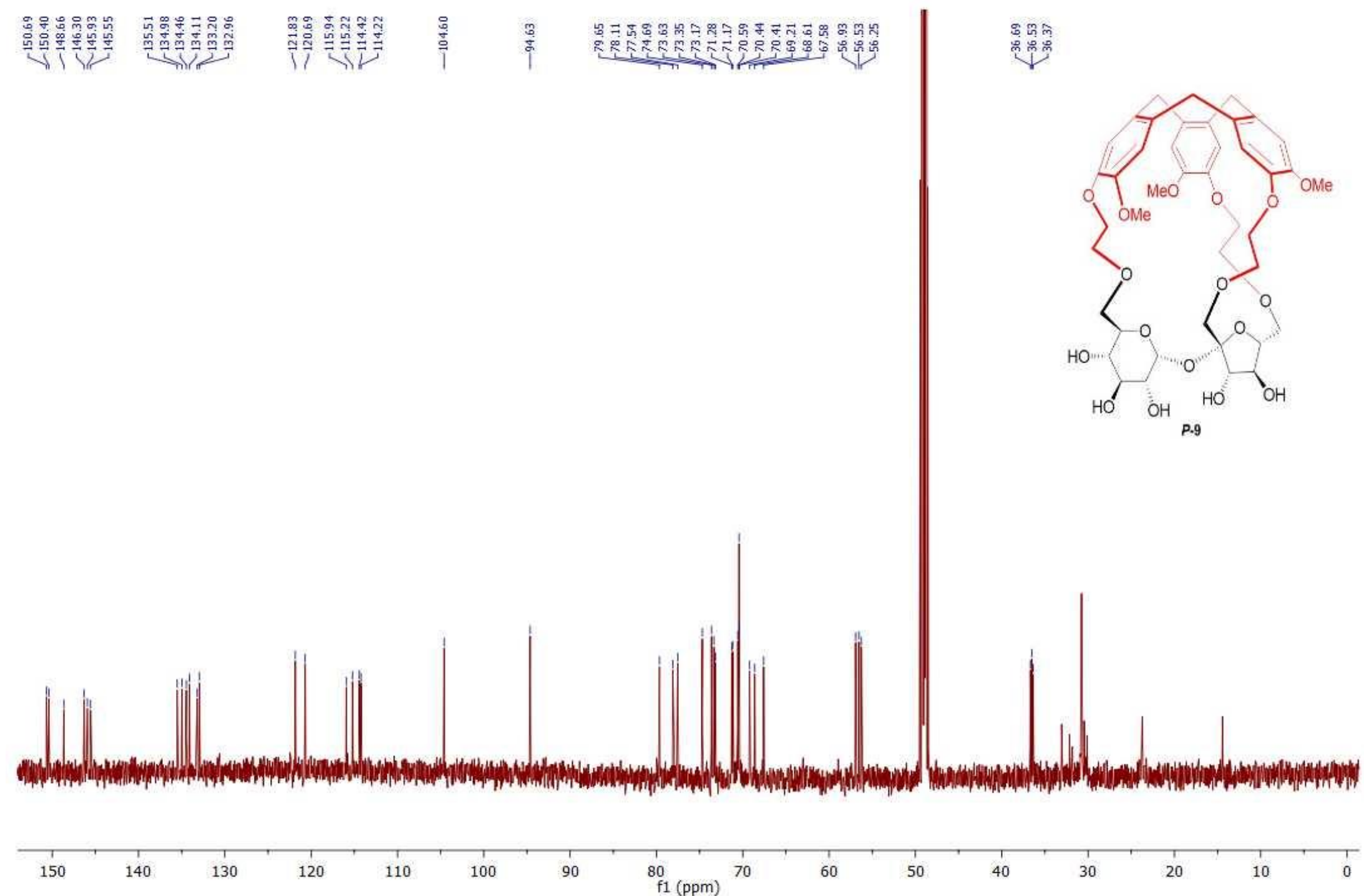

Figure S75. ${ }^{13} \mathrm{C}$ NMR spectrum of compound $\boldsymbol{P}-\mathbf{9}$. 


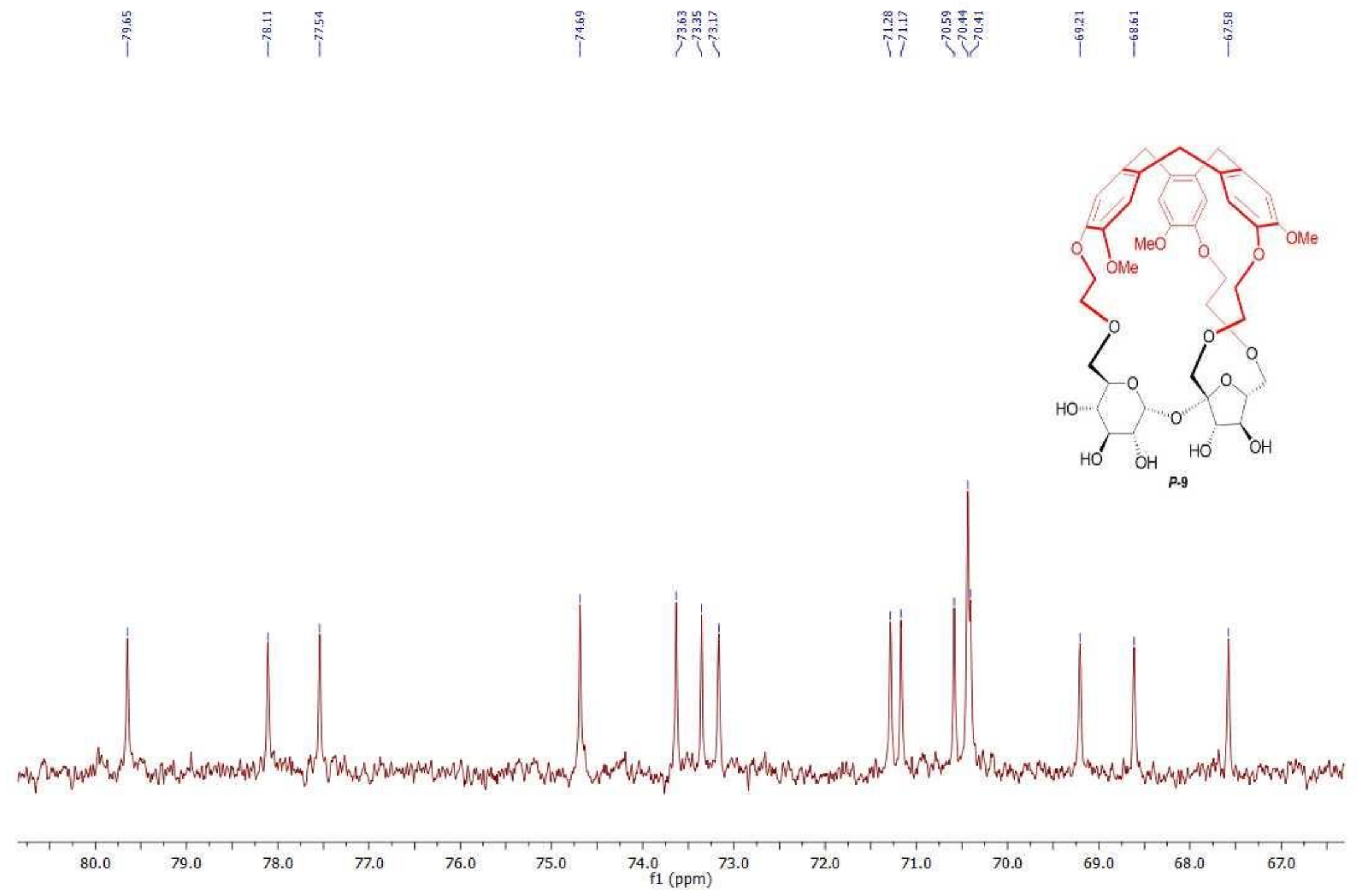

Figure S76. ${ }^{13} \mathrm{C}$ NMR spectrum of compound $\boldsymbol{P}-\mathbf{9}$ (aliphatic part). 


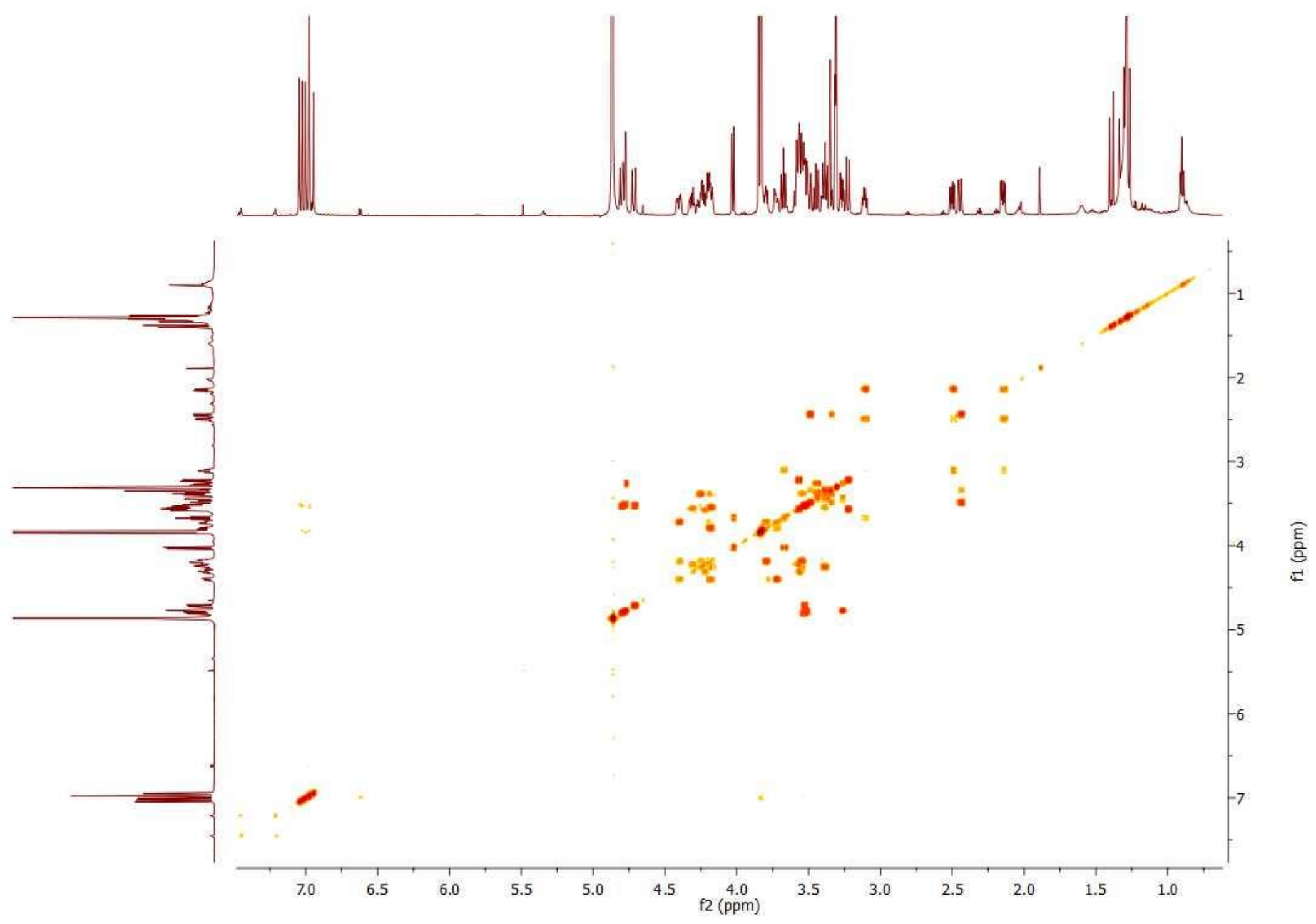

Figure S77. ${ }^{1} \mathrm{H}-{ }^{1} \mathrm{H}$ COSY spectrum of compound $\boldsymbol{P}-\mathbf{9}$. 


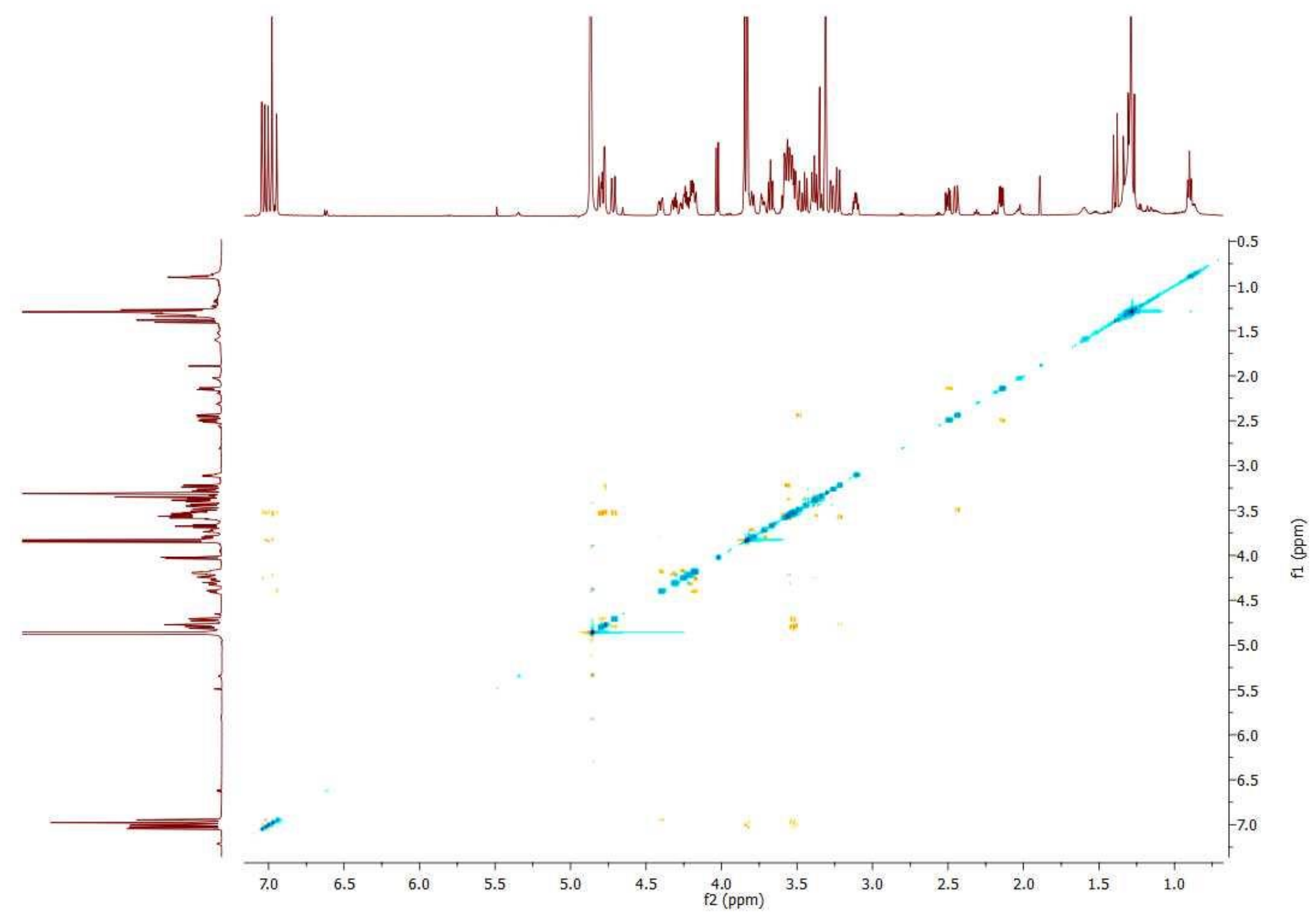

Figure S78. ${ }^{1} \mathrm{H}-{ }^{1} \mathrm{H}$ ROESY spectrum of compound $\boldsymbol{P}-\mathbf{9}$. 


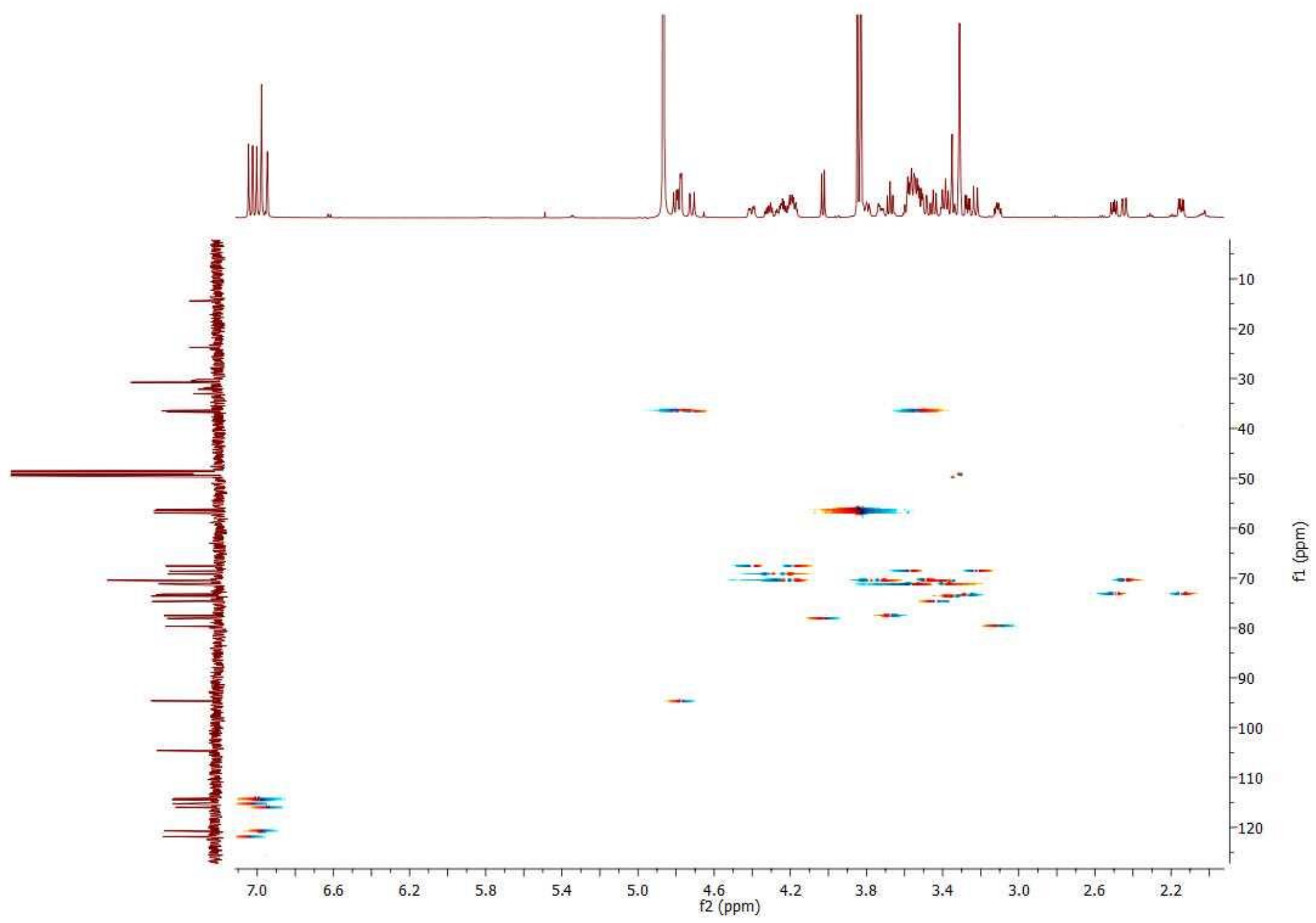

Figure S79. ${ }^{1} \mathrm{H}-{ }^{13} \mathrm{C}$ HSQC spectrum of compound $\boldsymbol{P}$-9. 


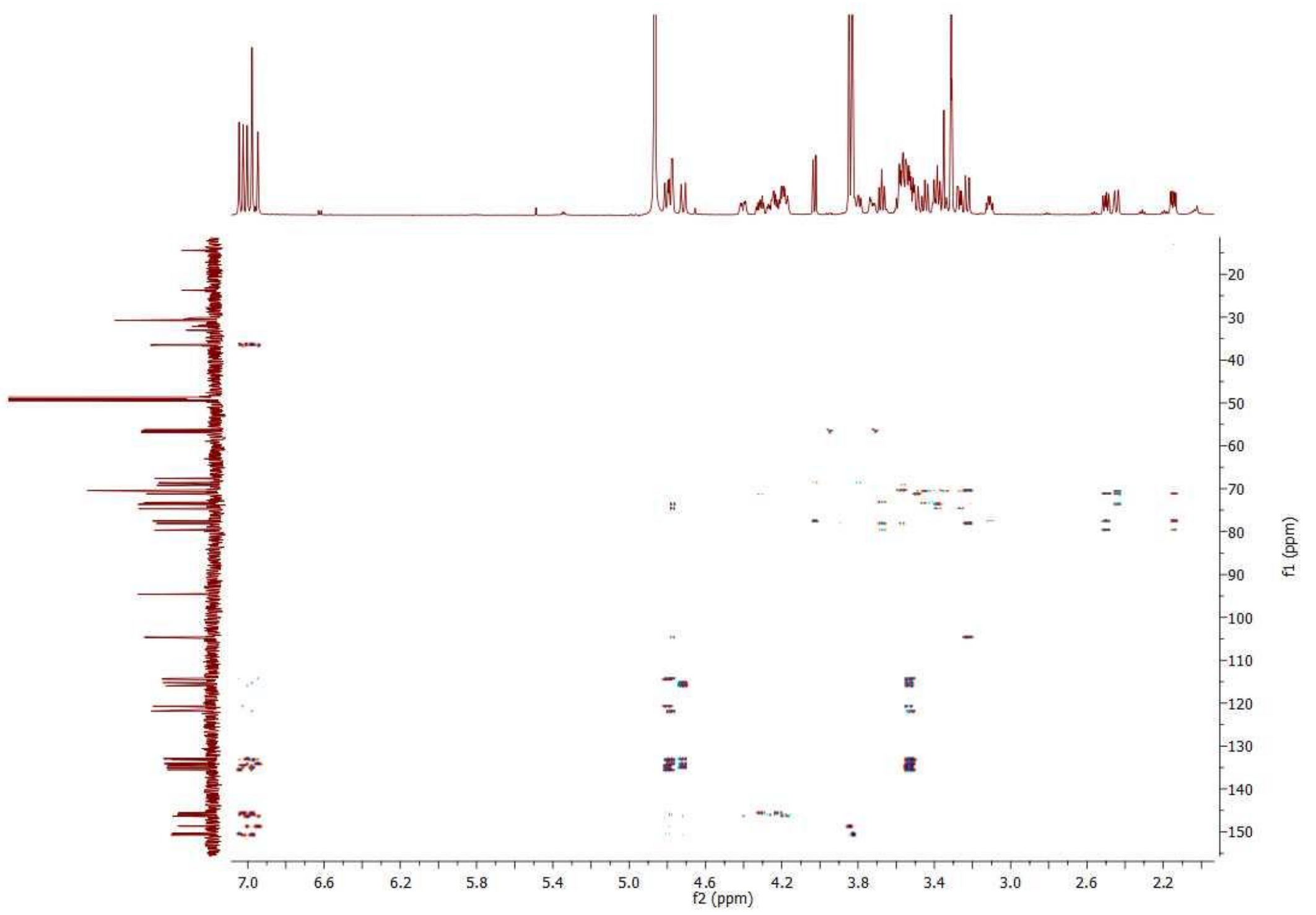

Figure S80. ${ }^{1} \mathrm{H}-{ }^{13} \mathrm{C}$ HMBC spectrum of compound $\boldsymbol{P}-\mathbf{9}$. 


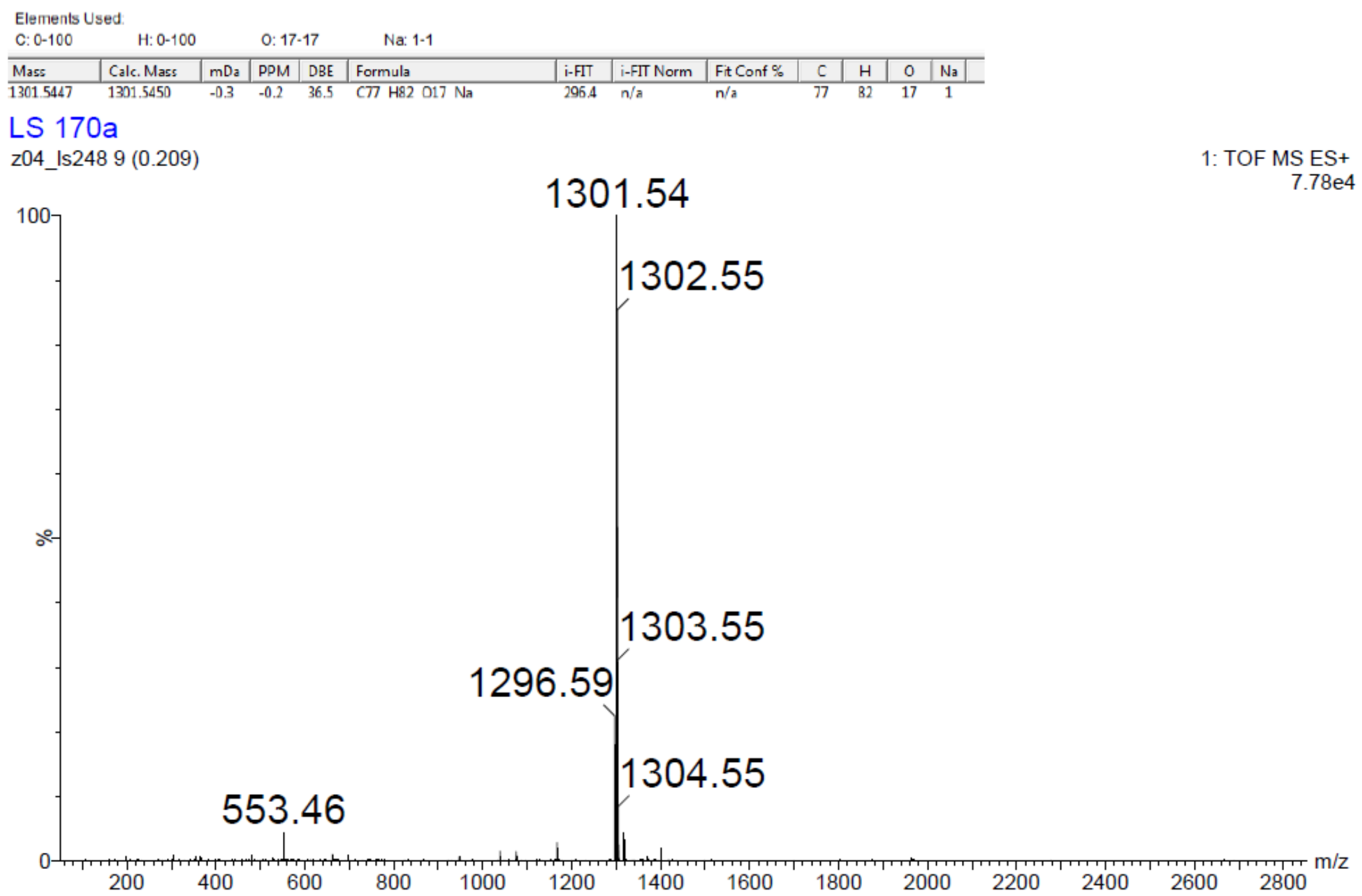

Figure S81. HRMS spectrum of compound $\boldsymbol{M}$-8. 


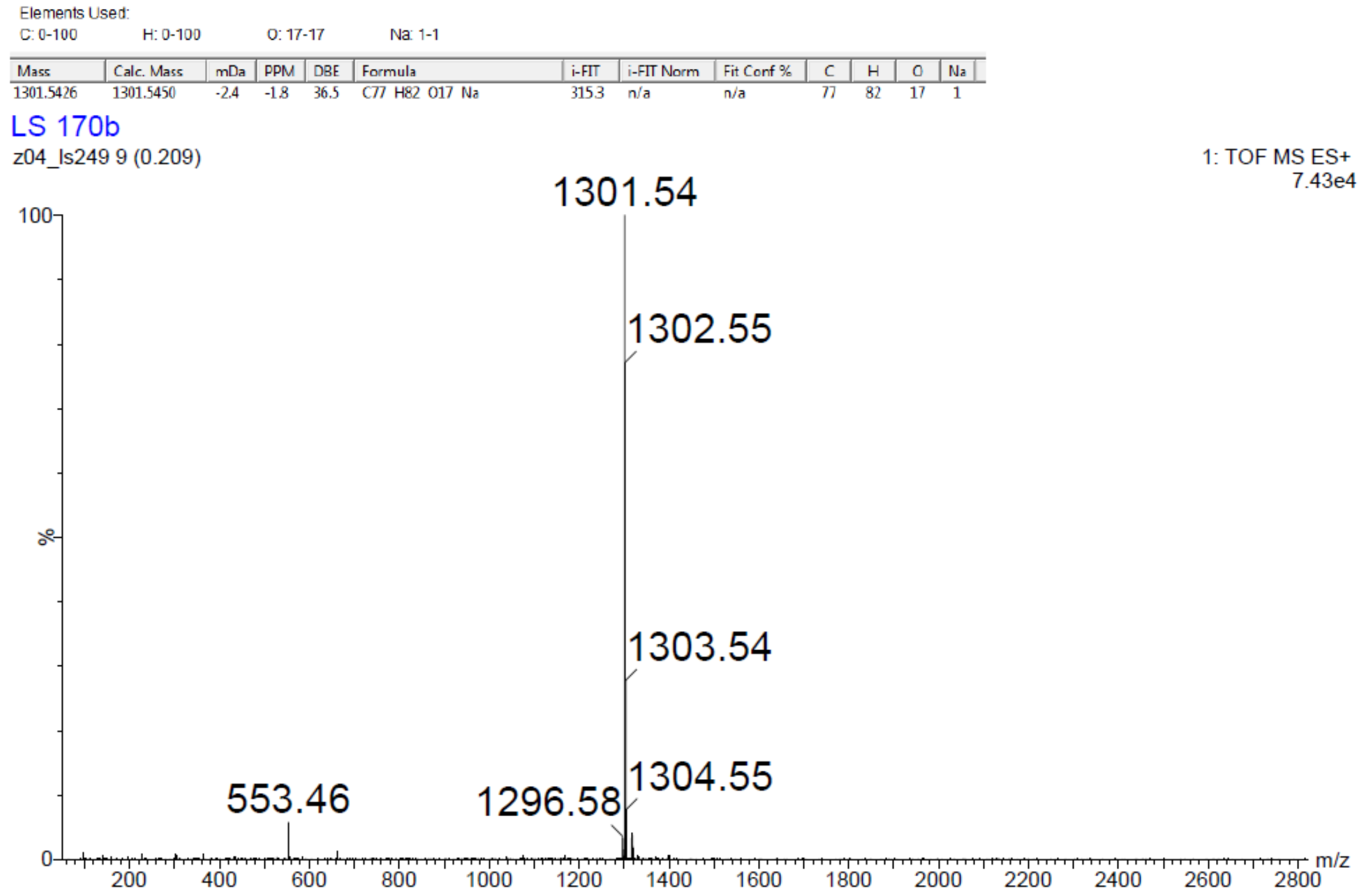

Figure S82. HRMS spectrum of compound $\boldsymbol{P} \mathbf{- 8}$. 


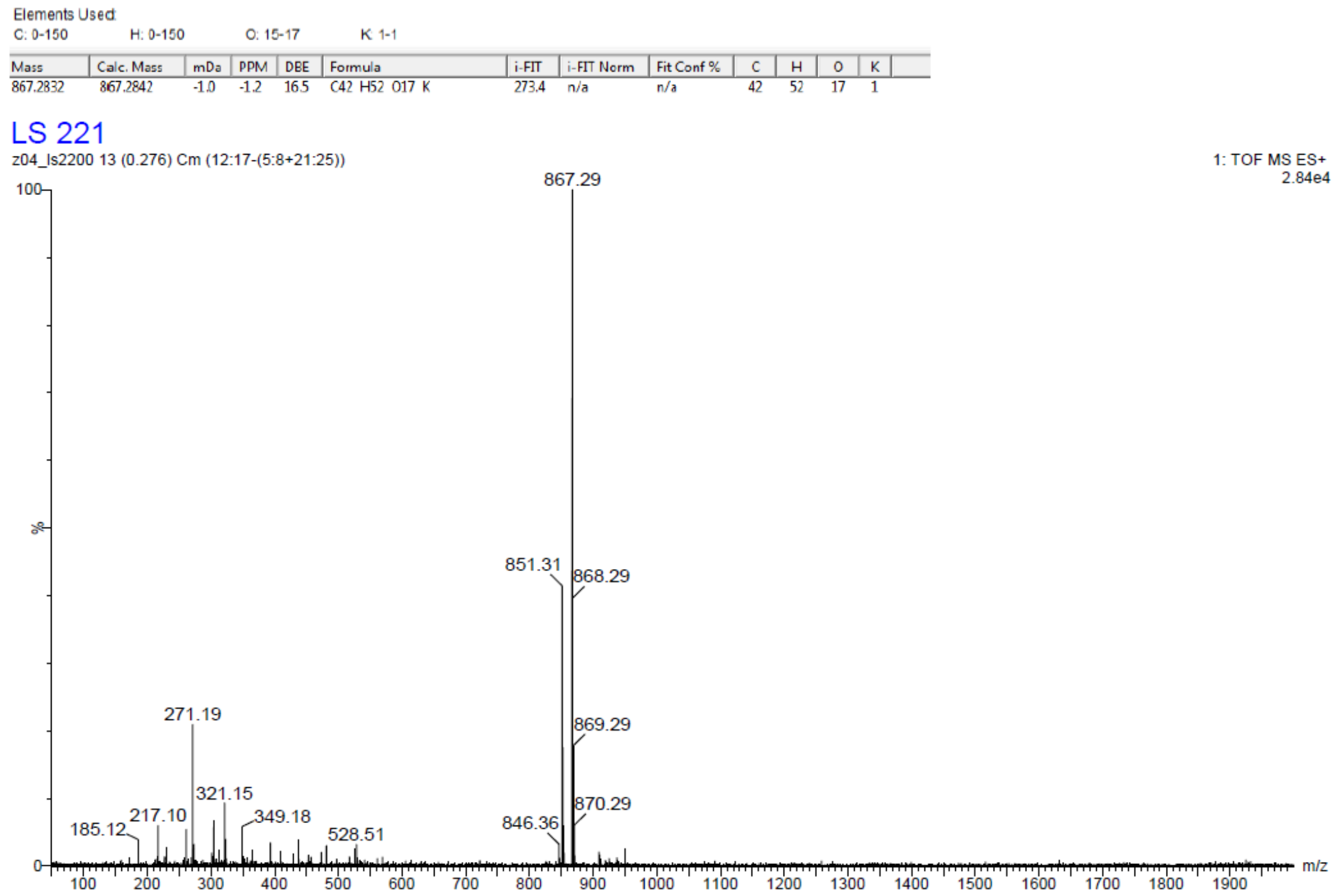

Figure S83. HRMS spectrum of compound $\boldsymbol{M - 9}$. 


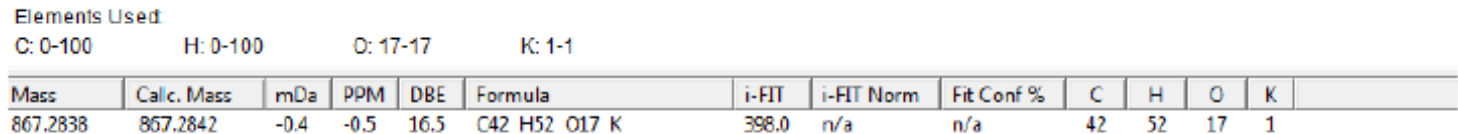

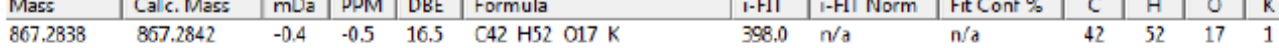

\section{LS198}

z04_Is1359 $10(0.226) \mathrm{Cm}(9: 12-(5: 8+14: 16))$

867.28

1: TOF MS ES+ $4.11 \mathrm{e} 4$

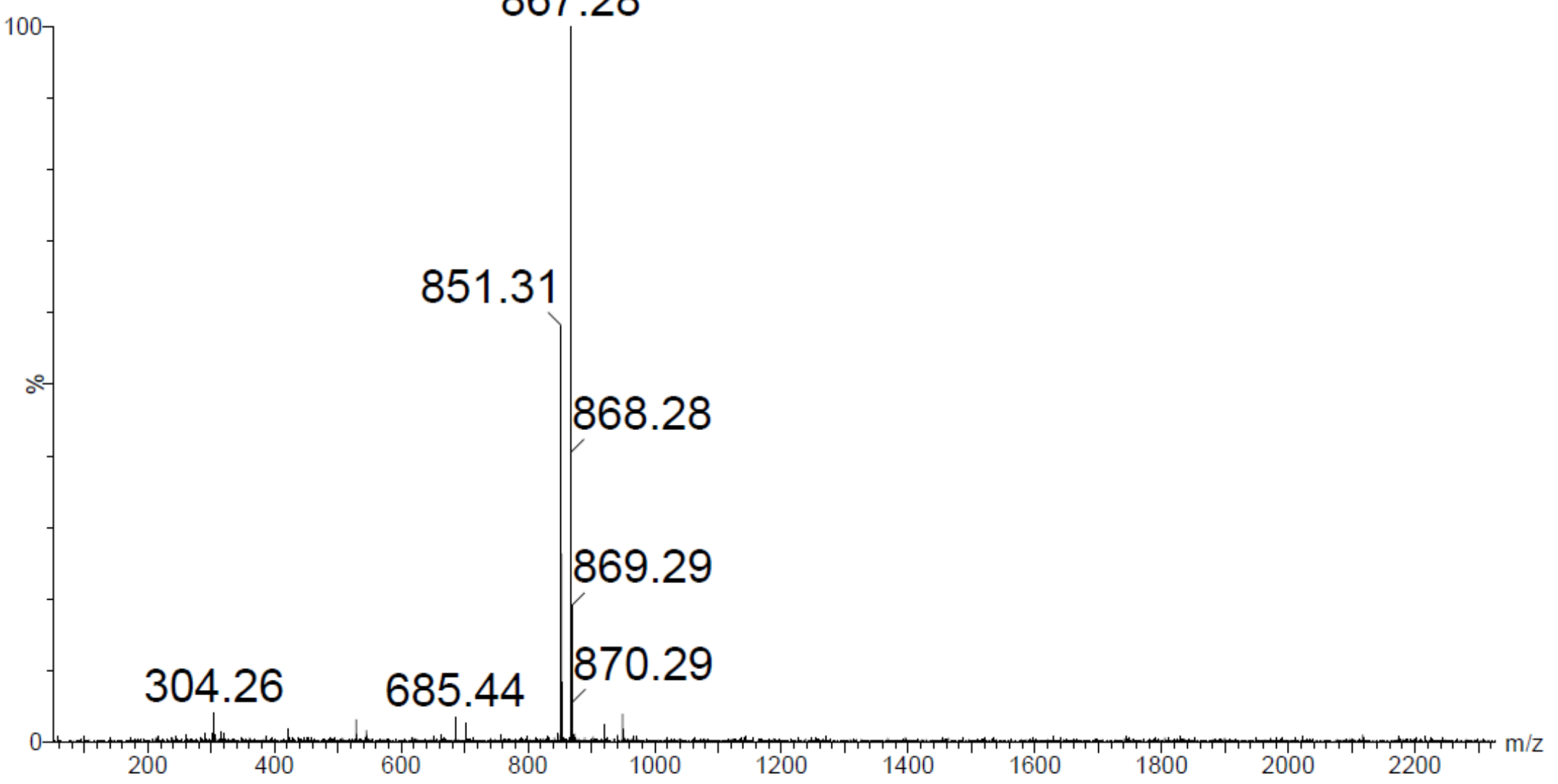

Figure S84. HRMS spectrum of compound $\boldsymbol{P}-9$. 
(1) CONFLEX 7; Conflex corporation.

(2) (a)Yanaia, T.; Tew, D.P., Handy, N.C. A new hybrid exchange-correlation functional using the Coulomb-attenuating method (CAMB3LYP). Chemical Physics Letters 2004, 393, 51-57. (b) Feller, D. The Role of Databases in Support of Computational Chemistry Calculations. J. Comp. Chem.1996, 17, 1571-1586. (c) Schuchardt, K.L.; Didier, B.T.; Elsethagen, T.; Sun, L.; Gurumoorthi, V.; Chase, J.; Li, J.; Windus, T.L. Basis Set Exchange: A Community Database for Computational Sciences. J. Chem. Inf. Model. 2007, 47, $1045-1052$.

(3) Gaussian 16, Revision B.01, Frisch, M.J.; Trucks, G.W.; Schlegel, H.B.; Scuseria, G.E.; Robb, M.A.; Cheeseman, J.R.; Scalmani, G.; Barone, V.; Petersson, G.A.; Nakatsuji, H.; Li, X.; Caricato, M.; Marenich, A.V.; Bloino, J.; Janesko, B.G.; Gomperts, R.; Mennucci, B.; Hratchian, H.P.; Ortiz, J. V.; Izmaylov, A. F.; Sonnenberg, J. L.; Williams-Young, D.; Ding, F.; Lipparini, F.; Egidi, F.; Goings, J.; Peng, B.; Petrone, A.; Henderson, T.; Ranasinghe, D.; Zakrzewski, V.G.; Gao, J.; Rega, N.; Zheng, G.; Liang, W.; Hada, M.; Ehara, M.; Toyota, K.; Fukuda, R.; Hasegawa, J.; Ishida, M.; Nakajima, T.; Honda, Y.; Kitao, O.; Nakai, H.; Vreven, T.; Throssell, K.; Montgomery, J.A., Jr.; Peralta, J.E.; Ogliaro, F.; Bearpark, M.J.; Heyd, J.J.; Brothers, E.N.; Kudin, K.N.; Staroverov, V.N.; Keith, T.A.; Kobayashi, R.; Normand, J.; Raghavachari, K.; Rendell, A.P.; Burant, J.C.; Iyengar, S.S.; Tomasi, J.; Cossi, M.; Millam, J.M.; Klene, M.; Adamo, C.; Cammi, R.; Ochterski, J.W.; Martin, R.L.; Morokuma, K.; Farkas, O.; Foresman, J.B.; Fox, D.J. Gaussian, Inc., Wallingford CT, 2016.

(4) Wei, Q.; Seward, G.K.; Hill, P.A.; Patton, B.; Dimitrov, I.E.; Kuzma, N.N.; Dmochowski, I.J.Designing ${ }^{129}$ Xe NMR Biosensors for Matrix Metalloproteinase Detection. J. Am. Chem. Soc. 2006, 128, 13274-13283.

(5) Brotin, T.; Devic, T.; Lesage, A.; Emsley, L.; Collet, A. Synthesis of Deuterium-Labeled Cryptophane-A and Investigation of Xe@Cryptophane Complexation Dynamics by 1D-EXSY NMR Experiments. Chem. Eur. J. 2001, 7, 1561-1573. 